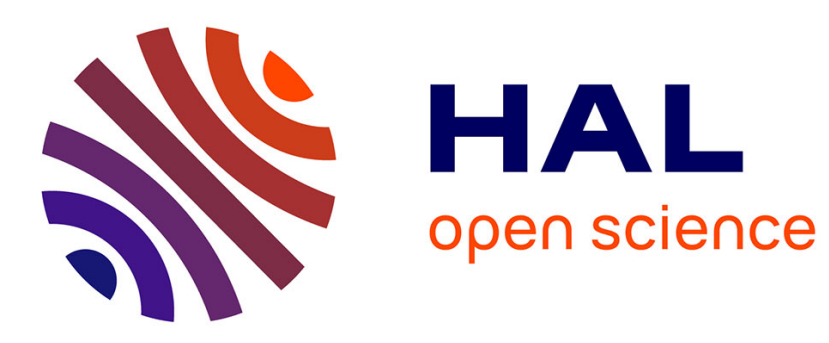

\title{
Essential facts about Covid-19: the disease, the responses, and an uncertain future. For South African learners, teachers, and the general public
}

Martin Bucher, Mall Anwar Suleman, Karim Quarraisha Abdool, Durbach Steven, Hansraj Australia Sudan, Hlabisa Bongeka, Majake Tsepo, Mendelson Marc, Petruccione Francesco, Phillips Howard, et al.

\section{To cite this version:}

Martin Bucher, Mall Anwar Suleman (Dir.). Essential facts about Covid-19: the disease, the responses, and an uncertain future. For South African learners, teachers, and the general public. 2021, 10.17159/assaf.2021/0072 . hal-03453998

\section{HAL Id: hal-03453998 https://hal.science/hal-03453998}

Submitted on 16 Dec 2021

HAL is a multi-disciplinary open access archive for the deposit and dissemination of scientific research documents, whether they are published or not. The documents may come from teaching and research institutions in France or abroad, or from public or private research centers.
L'archive ouverte pluridisciplinaire HAL, est destinée au dépôt et à la diffusion de documents scientifiques de niveau recherche, publiés ou non, émanant des établissements d'enseignement et de recherche français ou étrangers, des laboratoires publics ou privés. 
Essential facts about
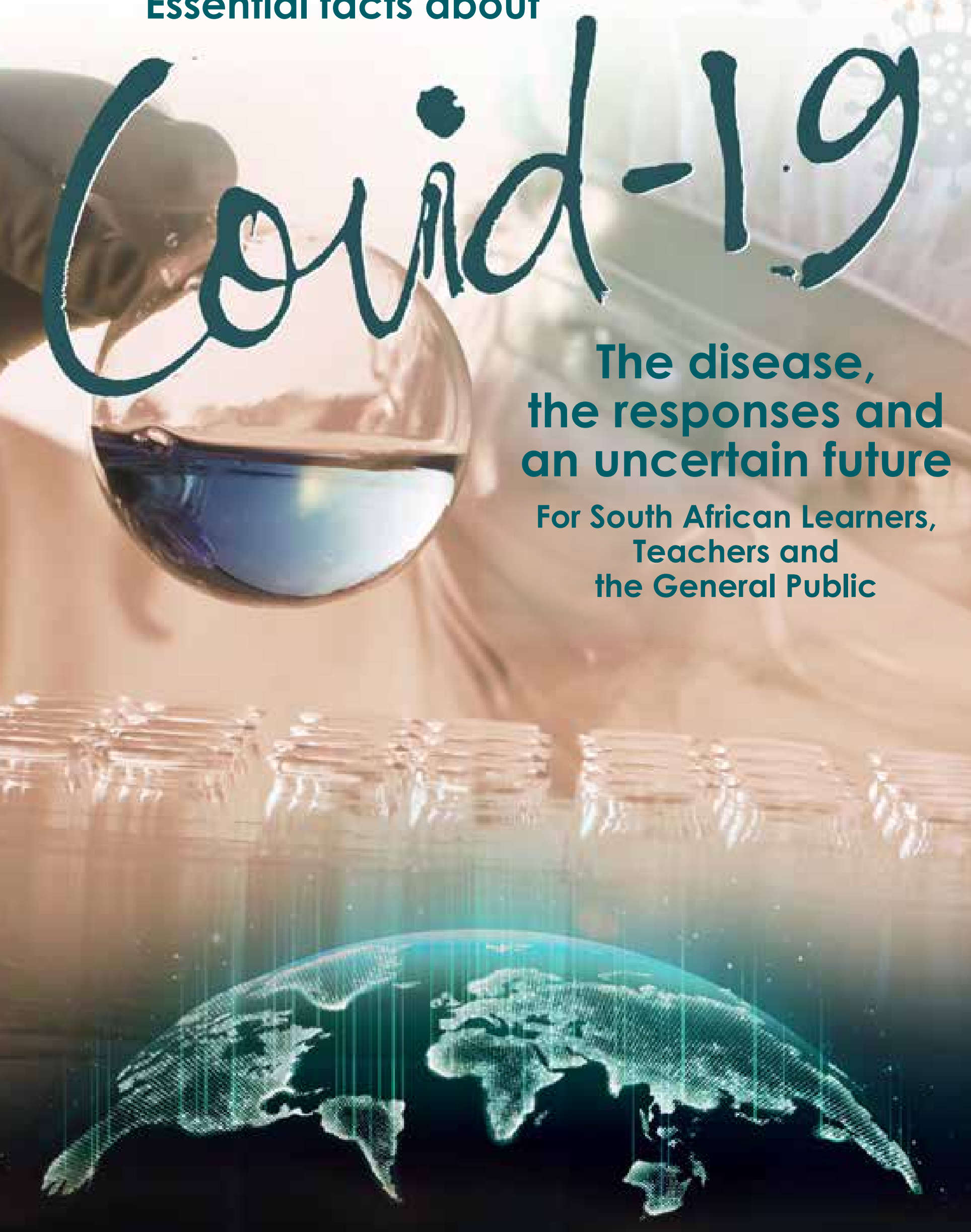

Commissioned by the Academy of Science of South Africa (ASSAf) 
(C) Academy of Science of South Africa

ISBN 978-1-928496-37-3

DOI http://dx.doi.org/10.17159/assaf.2021/0072

July 2021

Published by: Academy of Science of South Africa (ASSAf)

PO Box 72135, Lynnwood Ridge, Pretoria, South Africa, 0040

Tel: +27 123496600 • Fax: +27 865769520

E-mail: admin@assaf.org.za

Reproduction is permitted, provided the source and publisher are appropriately acknowledged.

The Academy of Science of South Africa (ASSAf) was inaugurated in May 1996. It was formed in response to the need for an Academy of Science consonant with the dawn of democracy in South Africa: activist in its mission of using science and scholarship for the benefit of society, with a mandate encompassing all scholarly disciplines that use an open-minded and evidence-based approach to build knowledge. ASSAf thus adopted in its name the term 'science' in the singular as reflecting a common way of enquiring rather than an aggregation of different disciplines. Its Members are elected on the basis of a combination of two principal criteria-academic excellence and significant contributions to society.

The Parliament of South Africa passed the Academy of Science of South Africa Act (No 67 of 2001), which came into force on 15 May 2002. This made ASSAf the only academy of science in South Africa officially recognised by government and representing the country in the international community of science academies and elsewhere.

https://www.assaf.org.za/

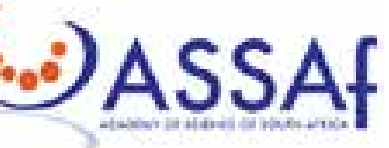




\section{EDITORS}

Martin Bucher and Anwar Suleman Mall

EDITORIAL TEAM

Jane Carruthers, Wieland Gevers, Tsepo Majake, Himla Soodyall, Lovise van Heerden, Susan Veldsman, Renate Venier, Henriette Wagener, Caradee Wright

\section{COPY EDITING}

Joanne Riley

\section{LAYOUT}

Pamela Benporath

\section{COLLABORATORS}

Michael Ellis and Joanne Riley, South African Agency for Science and Technology Advancement (SAASTA)

Kanthan Naidoo, Department of Basic Education

\section{CONTRIBUTORS}

Quarraisha Abdool Karim, Pro-Vice Chancellor for African Health, University of KwaZulu-Natal and Professor in Clinical Epidemiology, Columbia University, New York

Martin Bucher, Fractional Research Professor of Mathematics, UKZN and SARAO, and CNRS Directeur de Recherche, Université de Paris and Ecole Normale Supérieure, Paris, France

Steven Durbach, Former Lecturer, School of Molecular and Cell Biology, University of Witswatersrand, currently independent artist at interface of Art and Science in Sydney, Australia

Sudan Hansraj, Professor of Mathematics, University of KwaZulu-Natal

Bongeka Hlabisa, Research Scientist, CAPRISA, University of KwaZulu-Natal

Tsepo Majake, Education Liaison Officer, Academy of Science of South Africa

Anwar Suleman Mall, Emeritus Professor, University of Cape Town

Marc Mendelson, Professor of Infectious Diseases and Head of the Division of Infectious Diseases and HIV Medicine at Groote Schuur Hospital, University of Cape Town

Francesco Petruccione, Professor of Physics, University of KwaZulu-Natal Howard Phillips, Professor of History, University of Cape Town

Linda Richter, Distinguished Professor, DSI-NRF Centre of Excellence in Human Development, University of the Witwatersrand

Ed Rybicki, Professor of Microbiology, University of Cape Town

Wiedaad Slemming, Senior Lecturer, Division of Community Paediatrics, Department of Paediatrics and Child Health, University of the Witwatersrand

Mark Tomlinson, Co-Director, Institute for Life Course Health Research, Department of Global Health, Stellenbosch University

Alex van den Heever, Chair of Social Security Systems Administration and Management Studies, University of the Witwatersrand 
Martin Bucher

(1) https://orcid.org/0000-0002-9961-9772 Jane Carruthers

(D) https://orcid.org/0000-0001-8210-4599

Michael Ellis

(1) https://orcid.org/0000-0002-8946-1510

Wieland Gevers

(1) https://orcid.org/0000-0003-1 138-2625

Sudan Hansraj

(D) https://orcid.org/0000-0002-8305-7015

Quarraisha Abdool Karim

(D) https://orcid.org/0000-0002-0985-477X

Tsepo Majake

(1) https://orcid.org/0000-0003-3657-7314

Anwar Suleman Mall

(1) https://orcid.org/0000-0001-7737-6194

Marc Mendelson

(1) https://orcid.org/0000-0001-6668-3893

Francesco Petruccione

(1) https://orcid.org/0000-0002-8604-0913

Howard Phillips

(1) https://orcid.org/0000-0002-9035-2311

Linda Richter

(1) https://orcid.org/0000-0002-3654-3192

Ed Rybicki

(1) https://orcid.org/0000-0001-8024-9911

Wiedaad Slemming

(1) https://orcid.org/0000-0002-1566-8228

Himla Soodyall

(D) https://orcid.org/0000-0003-2488-9185

Mark Tomlinson

(1) https://orcid.org/0000-0001-5846-3444

Alex van den Heever,

(D) https://orcid.org/0000-0002-1421-0784

Susan Veldsman

(1) http://orcid.org/0000-0002-7557-0898

Caradee Wright

(1) https://orcid.org/0000-0001-9608-818X 


\section{CONTENTS}

1 PREFACE

3 FOREWORD

7 CHAPTER 1

Rise of Covid-19: The World Faces a New Pandemic

13 CHAPTER 2 Infectious Diseases in South African History

16 CHAPTER 3 Infectious Diseases in World History

27 CHAPTER 4

Quarantines, Social Distancing, and All That

38 CHAPTER 5

Dynamics of Epidemics

65 CHAPTER 6

Viruses in the Human Body

73 CHAPTER 7

Medical Aspects of

Covid-19

95 CHAPTER 8

Anti-Viral Drugs and Vaccines
108 CHAPTER 9 The South African Healthcare System and Covid-19

122 CHAPTER 10 Covid-19 and Secondary Education

130 CHAPTER 11 Covid-19 and the Economy

139 EPILOGUE

142 APPENDIX A Reliable Sources of Information and Further Reading

144 APPENDIX B Covid-19,Misinformation, Myths, and Fake News

148 APPENDIX C Scientific Method and Evidence-Based Medicine

154 GLOSSARY 

The first cases of a new coronavirus (SARS-CoV-2) were identified toward the end of 2019 in Wuhan, China. Over the following months, this virus spread to everywhere in the world. By now no country has been spared the devastation from the loss of lives from the disease (Covid-19) and the economic and social impacts of responses to mitigate the impact of the virus. Our lives in South Africa have been turned upside down as we try to make the best of this bad situation. The 2020 school year was disrupted with closure and then reopening in a phased approach, as stipulated by the Department of Education.

This booklet is a collective effort by academics who are Members of the Academy of Science of South Africa (ASSAf) and other invited scholars to help you appreciate some of the basic scientific facts that you need to know in order to understand the present crisis and the various options available to respond to it. We emphasise that the threat of infectious diseases is not an entirely new phenomenon that has sprung onto the stage out of nowhere. Infectious diseases and pandemics have been with us for centuries, in fact much longer. Scientists have warned us for years of the need to prepare for the next pandemic.

Progress in medicine in the course of the 20th century has been formidable. Childhood mortality has greatly decreased almost everywhere in the world, thanks mainly, but not only, to the many vaccines that have been developed. Effective drugs now exist for many deadly diseases for which there were once no cures.

For many of us, this progress has generated a false sense of security. It has caused us to believe that the likes of the 1918 'Spanish flu' pandemic, which caused some 50 million deaths around the world within a span of a few months, could not be repeated in some form in today's modern world.

The Covid-19 pandemic reminds us that as new cures for old diseases are discovered, new diseases come along for which we are unprepared. And every hundred or so years one of these diseases wreaks havoc on the world and interferes severely with our usual ways of going about our lives. Today's world has become increasingly interconnected and interdependent, through trade, migrations, and rapid air travel. This globalisation makes it easier for epidemics to spread, somewhat offsetting the power of modern medicine.

In this booklet we have endeavoured to provide an historical perspective, and to enrich your knowledge with some of the basics of medicine, viruses, and epidemiology. Beyond the immediate Covid-19 crisis, South Africa faces a number of other major health challenges: highly unequal access to quality healthcare, widespread tuberculosis, HIV infection causing AIDS, a high prevalence of mental illness, and a low life expectancy, compared to what is possible with today's medicine. It is essential that you, as young people, also learn about the nature of these new challenges, so that you may contribute to finding future solutions.

The Editors 
l-coronavirus entsha (i-SARS-CoV-2) ikhonjwa ngasekupheleni kuka-2019 endaweni yase Wuhan, China. Ezinyangeni ezilandelayo, leli gciwane lasakazeleeka kuyo yonke indawo emhlabeni. Okwamanje alikho izwe elisindile ekufenikwabantu ngenxa yalesi sifo (Covid-19), kanye nemithelela kwezomnotho, nakwezenhlalo ukunciphisa umthelela wegciwane. Izimpilo zethu eNingizimu Afrika zibebucayi njengoba sizama ukwenza okusemandleni kulesi simo esibi. Unyaka wesikole ka2020 waphazanyiswa ukuvalwa wabe uvulwa ngaphansi kwemigomo enezigaba, njengokusho koMnyango Wezemfundo. Le ncwajana iwumzamo ohlanganyelwe wezifundiswa ezingamalungu e-Academy of Science ENingizimu Afrika (ASSAf) nezinye izifundiswa ezimenyiwe ukukusiza ukuthi wazi ezinye zezinto eziyisisekelo, namaqiniso esayensi odinga ukuwazi ukuze uqonde ubunzima obukhona njengamanje.

Siyakugcizelela ukuthi usongo Iwezifo ezithathelanayo akusiyo into entsha ngokuphelele eye yavela kungazelelwe. Izifo ezithathelanayo nezifo eziwubhubhane bezinathi emakhulwini eminyaka edlule. Ososayensi basixwayisile iminyaka eminingi ngesidingo sokulungiselela ubhubhane olungalandela. Intuthuko kwezokwelapha kwiminyaka engaba yikhulu-namashumi amabili ibe nkulu kakhulu. Ukufa kwezingane kwehle kakhulu cishe yonke indawo emhlabeni, sibonga ikakhulukazi imithi yokugoma ekhiqiziwe. Imishanguzo esebenzayo manje kukhona izifo eziningi ezibulalayo. Kwabaningi bethu, lenqubekela phambili idale umuzwa ongewona wokuphepha. Kusenze sakholwa ukuthi kwakunjani ngobhubhane 'lomkhuhlane wasespain' Iwango-1918, olwabangela ukufa kwabantu abangaba yizigidi ezingama-50 umhlaba kungakapheli isikhathi sezinyanga ezimbalwa, ubungeke uphindwe ngendlela ethile esimweni sanamuhla umhlaba. Ubhubhane IweCovid-19 Iusikhumbuza ukuthi njengoba kutholakala ukwelashwa okusha kwezifo ezindala, okusha izifo ziza lapho esingazilungiselele. Futhi njalo eminyakeni eyikhulu noma ngaphezulu eyodwa yalezi izifo zizolimaza umhlaba futhi ziphazamisa kakhulu izindlela zethu ezijwayelekile zezimpilo.

Izwe lanamuhla seliya ngokuya lixhuma futhi lincikene, ngokusebenzisa ukuhweba, ukufuduka, nokuhamba emoyeni okusheshayo. Lokhu kubumbana kwembulunga yonke kwenza kube lula ukuthi ubhubhane lusabalale, ngandlela thile ukucisha amandla ezokwelapha zesimanje. Kule ncwajana sizame ukunikeza umbono womlando, nokunothisa ulwazi Iwakho ngezinye izisekelo zemithi, amagciwane, kanye nezifo ezithathelanayo. Ngaphandle kwenkinga esheshayo ye-Covid-19, iNingizimu Afrika ibhekene nezinye izinselelo ezinkulu zezempilo: kakhulu ukufinyelela ngokungalingani ekunakekelweni kwezempilo okusezingeni, isifo sofuba esandile, ukutheleleka ngegciwane lenculazi, Kanye nokwanda kakhulu kwezifo zengqondo. Kubalulekile ukuthi nina, njengabantu abasha, nifunde ngemvelo nezinselelo ezintsha, ukuze nibambe iqhaza ekutholeni izixazululo ngezikhathi ezizayo. 


\section{FOREWORD}

Educators are still divided on the relative merits of systematically imparting the basic principles of established disciplines as opposed to creating favourable conditions in which learners can flexibly explore how the tools of different disciplines can be used in context, to understand one big and highly relevant problem area 'in the round', so to speak.

The first approach reflects an older world in which young people were required to undergo formal apprenticeships in trade or craft guilds, a model which then evolved into the kind of discipline (or 'subject')-based schooling that became general in most high schools all over the world. The second is more suited to the modern world, where knowledge is expanding quickly, where adaptability and versatility are essential, where individuals can have many careers in one lifetime, and where most enterprises require teamwork. (I should add that ultimately there is no substitute for

This booklet is based squarely on the contextual exploration of a multi-sided topic that is affecting every one of us, the great Covid-19 pandemic of late 2019 onwards to the present time and beyond. specialists being fully trained in their own discipline to be effective practitioners, but it is also helpful if they have already learnt to study things 'in the round' and to work across disciplinary lines.)

This booklet is based squarely on the contextual exploration of a multi-sided topic that is affecting every one of us, the great Covid-19 pandemic of late 2019 onwards to the present time and beyond. It assumes that motivation amongst learners to explore the topic will be high, as to understand it more fully is actually necessary in order to survive, as individuals, families, communities and as a nation, itself within the community of nations that represents humankind. There is history here, as well as the biology of pathogens and hosts; applied mathematics and epidemiology; human and animal medicine; psychology; economics; ethics; and politics. Everything hangs together and can't be understood in isolation. It is ONE problem, ONE crisis, and the tools of many disciplines have to be applied to all its facets.

When the great 'Cold War' ended in 1989, forty-four years after World War Two had ravaged much of the earth, most people hoped that the 'old world' of mindless nationalism, wars, and destructive competition would be replaced by a cooperative 'new world' of shared, cooperative institutions such as the United Nations, the World Health Organisation, the World Trade Organisation, the International Criminal Court, 
and many others. For example, the Paris Accord on Climate Change would address this most serious long-term problem cooperatively, all shoulders to the same wheel. The food supply could be optimised so that few people would be hungry and global health would be promoted. People would get to know 'the other' and see them as friends and not as enemies or rivals. (Strange how these aspirations resemble the collective and integrated way in which a major global disaster such as Covid-19 has to be addressed, as described above.)

A very striking example of 'world cooperation' has been the decision of all science publishers across the globe to make all articles dealing with Covid-19 freely available on the internet to every reader, and to speed up the publication of new findings in various new ways. It has provided a welcome glimpse of the kind of equal open access to knowledge that scientists and the public have long sought.

In recent years, many nations have unfortunately fallen back on outmoded ways of selfishly thinking about themselves in, or rather against, the world and this has also disappointingly surfaced in their responses to their local versions of the pandemic. Yet anybody reading this booklet cannot fail to see how wrong it is to be selfish at any level in a global pandemic caused by a highly infectious and damaging virus that has its own ways of overcoming everything we throw at it. Team Humankind has its work cut out to win this struggle. You, the reader of this booklet, are part of the team. Knowing what you can learn in this booklet will enable you to play an active role and to avoid falling into traps of misinformation, confusion and group hysteria.

Enjoy the read-you will learn a great deal that will be both useful and very interesting. And who knows, in your life ahead, you may have reason to be very thankful that you took the trouble to be well-informed about this pandemic, when the next one strikes the world.

\section{Wieland Gevers}

Emeritus Professor of Medical Biochemistry, University of Cape Town;

President of ASSAf, 1998-2004.

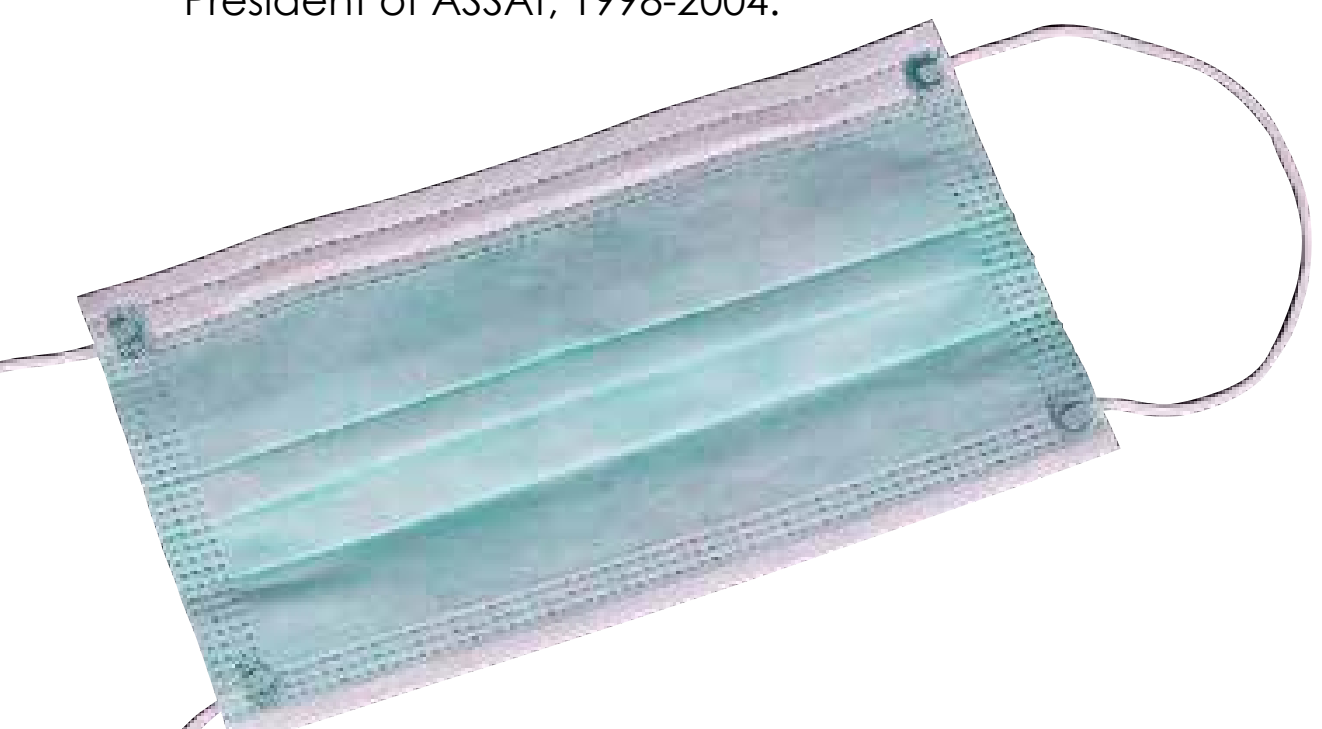





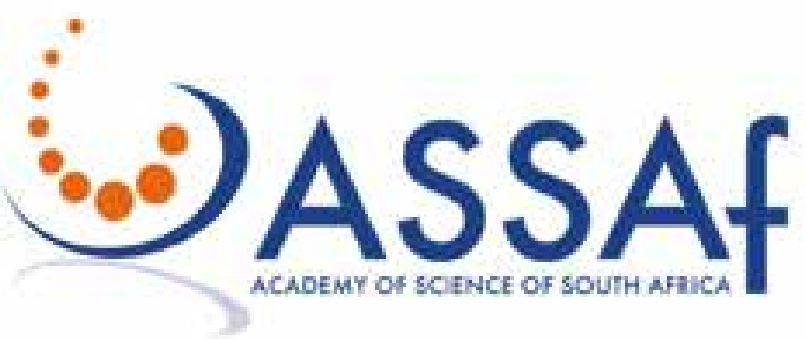

Applying scientific thinking in the service of society 


\section{CHAPTER 1}

The Rise of Covid-19: The World Faces a New Pandemic

The world has been facing a coronavirus pandemic since late 2019 and it shows no sign of abating. Some countries are experiencing a second wave of infections. The pandemic began in China and spread all over the world. Some governments, like the South African government, in consultation with scientists, took immediate steps to halt the spread of the virus. These involved an immediate lockdown (beginning 26 March 2020), including behavioural interventions such as the wearing of masks, regular hand-washing and social distancing, curfews, the banning of gatherings and the sale of cigarettes and alcohol, the latter in preparation for our health services to be ready to deal with a health crisis. As of 1 July 2021, South Africa has suffered over 60000 Covid-19 deaths.

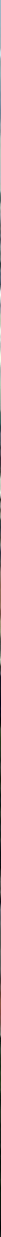




\section{Beginnings of the Pandemic}

By now almost everyone has heard of Covid-19. The Covid-19 pandemic has changed our daily lives and will continue to do so in the coming months and likely much longer.

The contagious diseases that are already well-known and regularly spread around communities, nations, regions and sometimes globally (the last are called pandemics) are not the same forever. New diseases emerge from time to time as disease-causing microbes evolve by changes in their genetic code. These microbes, which you can only see with a microscope, can be viruses, bacteria, or other micro-organisms. Some of these changes give microbes the ability to infect new species, for example jumping from animals to humans. Further evolution of the genes may then occur to enable the organism to jump from one human host to another, creating the spreading situation we call an epidemic.

Thus, new contagious diseases arise by evolution as the disease-causing organisms adapt to their environment by natural selection, as originally described by Charles Darwin. The organisms will continue to evolve over the course of an epidemic as new mutations arise, and may be selected for, during the rapid reproductive cycles of the organisms in their infected human hosts.

In the case of Covid-19, it is believed that the disease-causing micro-organism, a human virus of the family called coronaviruses, originated from bats, likely through some other intermediate species of wild animal, maybe the pangolin.

\section{Outbreak in Wuhan}

Around December 2019 hospital doctors in Wuhan, China, observed the arrival of a large number of patients suffering from an

Since there was a number of other respiratory diseases going around at the time, it took a little while for doctors to recognise the connections between these cases and to conclude that they were dealing with a new disease. atypical pneumonia. These cases resembled each other, but they did not fit any known pattern. Since there was a number of other respiratory diseases going around at the time, it took a little while for doctors to recognise the connections between these cases and to conclude that they were dealing with a new disease. An important clue was that many patients were in one way or another connected to the Wuhan Huanan Seafood Wholesale Market, where many live animals other than fish are slaughtered and sold. However, there is some evidence that the Covid-19 virus could have originated from a lab accident, and this hypothesis cannot be ruled out. The origin of this new virus remains under investigation. 
At the end of that month, a team from the China Centre for Disease Control (CDC) was dispatched to Wuhan to investigate the outbreak and the World Health Organization (WHO) was notified. Known diseases such as SARS, MERS, and a number of other viral infections were quickly ruled out, and by 7 January the cause was determined to be a new coronavirus, initially called 2019-nCoV (for novel coronavirus) and now known as SARS-CoV-2 (Severe Acute Respiratory Syndrome caused by the new version of CoronaVirus 2). The genome of this RNA-virus was rapidly decoded (its complete base sequence determined) and a sensitive, but unfortunately slow and expensive, genetic test was developed.

Initially the risk of spread had been underestimated, but after the number of cases exceeded 500, the Chinese government imposed drastic measures, limiting the movement of people in and out of Wuhan, closing markets, restaurants and parks, and isolating people suspected of being infected in large makeshift hospitals. These kinds of strict and compulsory infection-containment measures have become known by the rather vague metaphoric term 'lockdown'.

The number of daily new cases peaked in mid-February at around 700 per day, but when the number had dropped to around 20 per day, China partially lifted the lockdown outside of Wuhan, and on 8 April 2020 the lockdown in Wuhan was

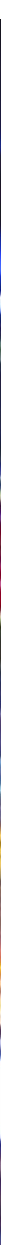


lifted as well. By the end of the lockdown, mainland China had experienced 81,907 confirmed infections, mostly concentrated in Wuhan and surrounding areas of the Hubei province, although the disease had also spread to every province in China.

\section{Spread of Covid-19 around the world}

Unfortunately, the spread of the disease did not stop in China. By now Covid-19 has spread to every continent of the world - and to every country. Different countries have adopted different strategies for responding to the disease, with varying success. What the right strategy is has not become completely clear, and it is very likely that the different context of each country may mean that no single strategy could be applied successfully everywhere. Some countries have been initially successful, only to see a second wave reappear.

Many countries closed their borders, hoping this would suffice to keep the disease out. Many did not prepare well and were faced with numbers of serious cases so large that hospital workers and other essential workers could not get enough masks and other personal protective equipment (PPE). There were also shortages of tests and testing supplies, making it impossible to decide whom to isolate.

\section{Covid-19 arrives in Europe and in the US}

After China, the epicentre of the disease spread to Europe. On 31 January 2020, the first Covid-19 cases in Europe were recorded in Rome, and shortly thereafter the disease spread to northern Italy, France, Spain, the United Kingdom, and elsewhere. By 13 March, the number of cases in Europe exceeded that of China. On 4 March, Italy 'locked down'. They took steps to close all sites where people were in close contact, including schools, universities, factories, concert halls, and recreational facilities. They were followed by France, Spain, the UK, and other countries shortly thereafter. In many parts of Europe, hospitals overflowed, especially in the intensive care units. Patients had to be transported elsewhere where there was capacity for treatment, and hospitals had to be reorganised. As the result of 'lockdowns', Europe managed to bring its numbers down, although many countries have experienced second, third, and further waves.

On 20 January 2020, the US saw its first confirmed case, and shortly thereafter the country banned travel from

Many countries closed their borders, hoping this would suffice to keep the disease out. China. But little else was done to prepare for a possible spread of the epidemic within the US, and the first test kit developed in the US proved to be defective. Starting in mid-March, the daily number of new cases in the northeastern states began to climb exponentially, especially in New York, where the peak number of cases reached 10,000 per day and the daily number of deaths reached around 1,000 per 
day around the beginning of April. Hospitals were overflowing and hospital workers lacked PPE causing many caregivers to become infected. New York Governor, Andrew Cuomo, declared a state of emergency on 13 March and, shortly thereafter, banned public gatherings, later (on 22 March) issuing a 'shelter-in-place order'. As cases in New York and neighbouring states of the US northeast subsided, cases grew in other areas of the country initially less affected. On 15 September 2020, the United States were leading the world with 6.7 million confirmed cases and 200,000 deaths, with India close behind with 5 million confirmed cases and a higher growth rate.

\section{Covid-19 reaches Africa}

Africa is a relative latecomer, with the first infections occurring in mid-February 2020. The first cases in Africa occurred in Egypt and in Nigeria, and the first case in South Africa, reported on 5 March 2020, involved a traveller returning from Italy. The early cases all involved people returning from abroad but soon it became apparent that 'community transmission' was taking place. In other words, people were becoming infected from others within South Africa rather than bringing the disease, one-byone, from abroad. Initial hopes of being able to stem the spread simply by restricting entry into the country and closely following up those testing positive were dashed.

On 23 March, a national 'lockdown', taking effect on 26 March, was announced by President Cyril Ramaphosa. Under this 21 -day lockdown all persons not taking part in 'essential services' were ordered to stay at home. Gatherings were banned, and

\section{Daily new confirmed COVID-19 cases}

Shown is the rolling 7-day average. The number of confirmed cases is lower than the number of actual cases, the main reason for that is limited testing.

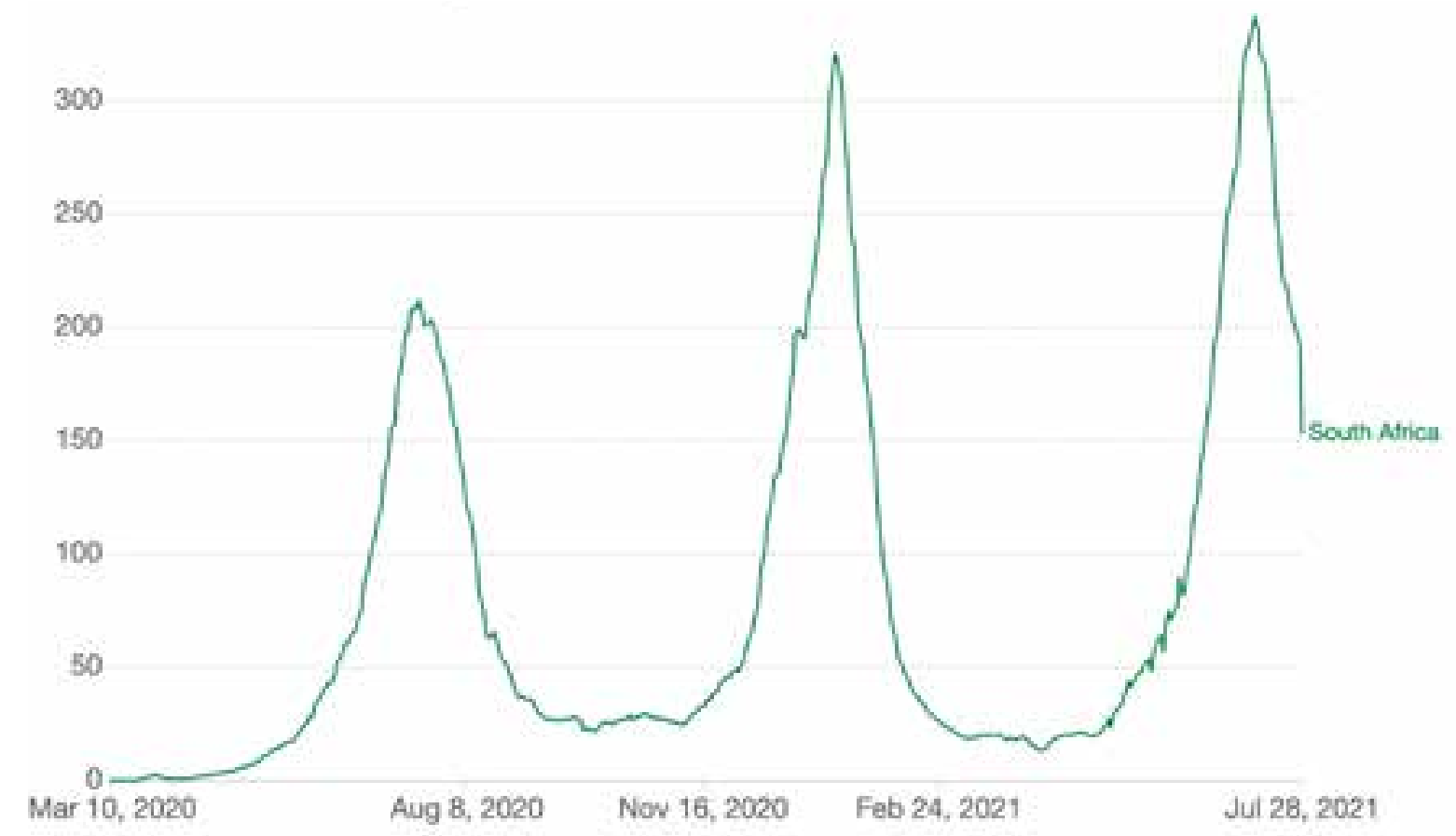

Figure 1.1: New daily cases

Source: https://ourworldindata.org/covid-cases. Creative commons license. 
factories, stores, bars, and restaurants were ordered closed. Travel in and out of the country (except for cargo) as well as between provinces was banned. The idea was to stop the spread of the disease and also to buy time, so that hospitals could prepare and reorganise to receive the large number of Covid-19 patients expected. Alcohol was banned to reduce the number of people coming to hospitals for alcohol related injuries, such as car accidents, and thus allow hospitals to concentrate on Covid-19 patients.

Despite measures put in place to lessen the economic impact of people not being able to work, maintaining the 'lockdown' proved challenging and also generated quite a lot of controversy. Representatives from various business groups argued that they should be exempted from the restrictions, and at times the debate on the details seemed to overlook the overall challenges presented to the country by the pandemic. Starting on 1 May, these restrictions began to be lifted gradually. Deciding the right balance between public health and economic survival has proved difficult, not just in South Africa but almost everywhere throughout the world.

Despite measures put in place to lessen the economic impact of people not being able to work, maintaining the 'lockdown' proved challenging and also generated quite a lot of controversy.

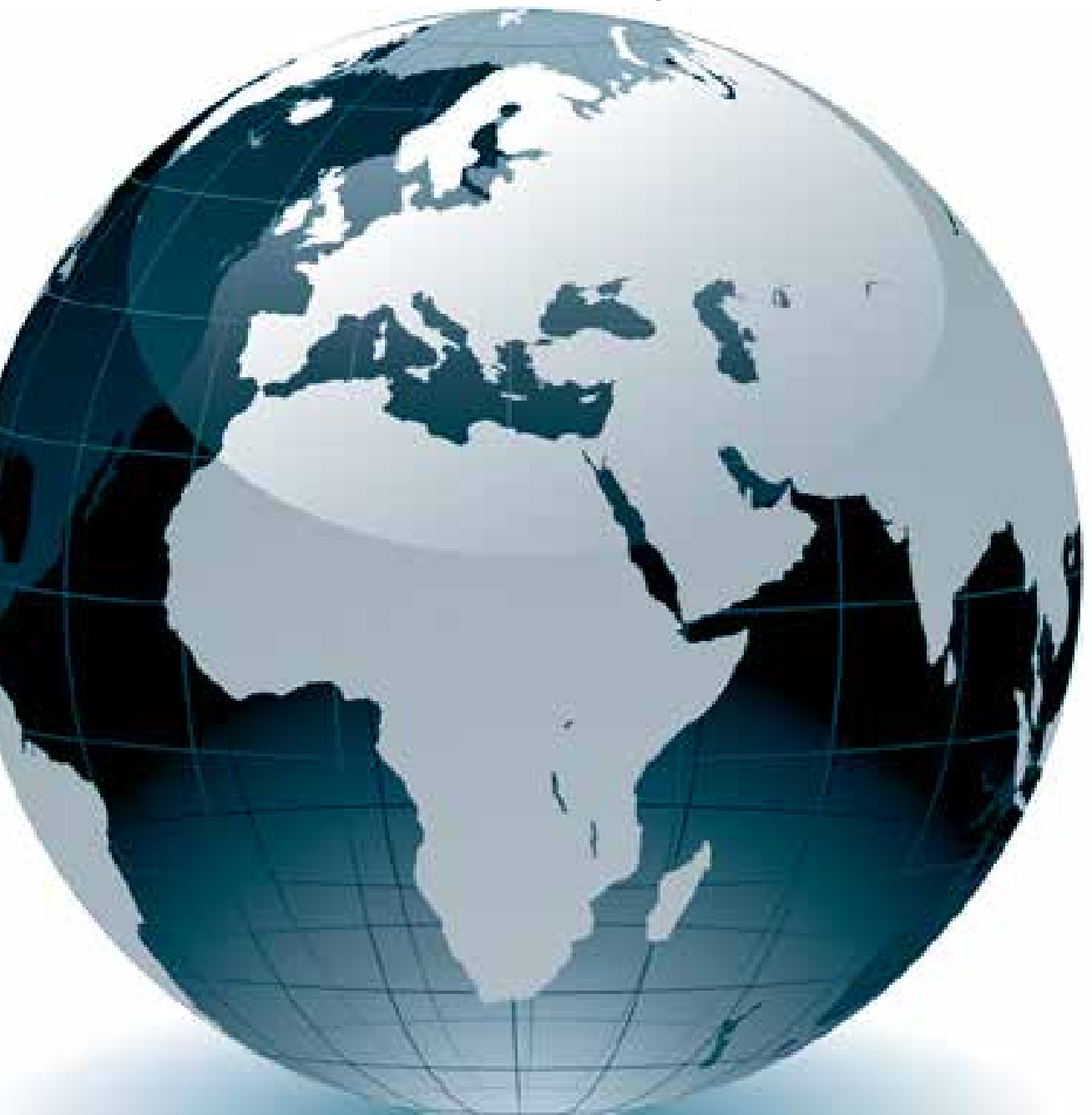

After the easing of the 'lockdown', the number of new daily cases started to greatly expand, reaching a peak in mid-July and dropping somewhat after that time (see Figure 1.1), and then reaching a second peak in early January 2021. 


\section{CHAPTER 2}

Infectious Diseases in South African History

Pandemics are not new to South Africa. Our country has experienced several pandemics that have swept the world in modern history, affecting humans and animals, examples of which are given below. The pandemic before Covid-19 freshest in our minds is HIV-AIDS, which, even though it was worldwide, hit sub-Saharan Africa the hardest, with KwaZulu-Natal recording the highest numbers in the region. It is worth remembering that attempts to find a preventive vaccine failed, but medication in the form of antiretrovirals helped sufferers lead normal lives.

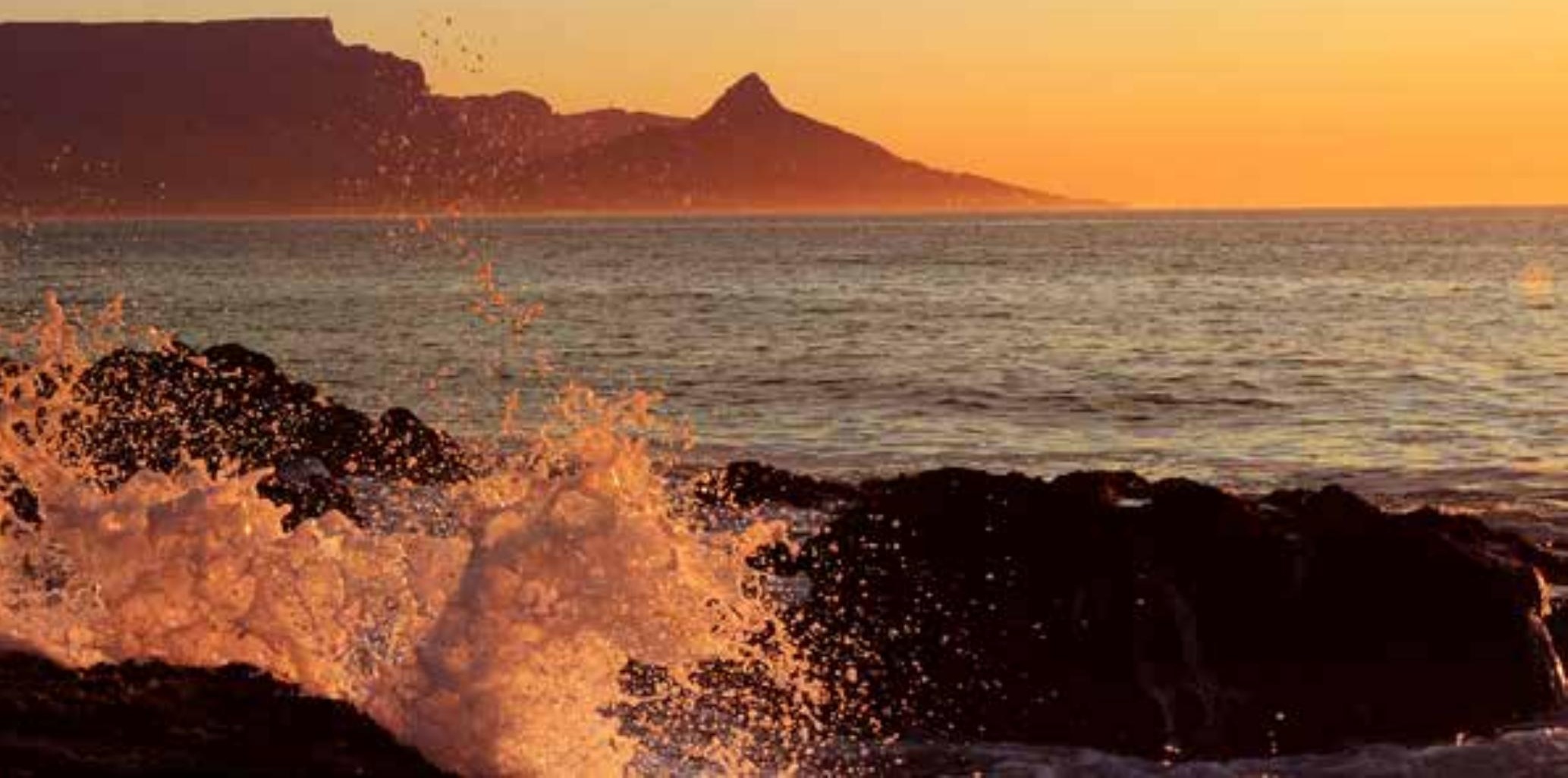


South Africa is no stranger to dire pandemics, which have claimed hundreds of thousands of lives as they have rampaged through the sub-continent. The worst of these were smallpox, which hit the region in eight recurrent waves between 1713 and 1893; plague in 1901-1904; the so-called 'Spanish' flu in 1918-1919; polio in 19441957; HIV/AIDS since 1982 and still widespread today; and, currently, Covid-19. There have also been pandemics affecting farm animals, such as the rinderpest amongst cattle that raged in the 1890 s.

All of these pandemics arrived in southern Africa from outside, either by sea, land, rail, or air, and were then spread by the movement of infected individuals across the land. South Africa in particular has a long history of people on the move, whether sailors, soldiers, war refugees, trekkers, truckers, fortune-seekers, migrant workers, railway staff, religious pilgrims, or tourists. If infected, they often became witting or unwitting vectors, with some being asymptomatic, as often in the case of Covid-19.

These pandemics had a severe demographic effect on the size and makeup of the population. In the case of smallpox, the Khoekhoen population was decimated to the point of having their social structures

Pandemics have also fuelled existing negative trends and attitudes, most obviously in prompting stigmatisation and furthering racial segregation. and independent existence shattered for all time. A contemporary spoke of them dying "by hundreds, so that they lay everywhere along the roads as if massacred as they fled inland with kraals, huts, and cattle". Devastating were the 'Spanish' flu and AIDS pandemics, both being especially lethal to young adults who made up the core of the country's labour force and the parents of the next generation, who were thereby turned into orphans on a massive scale. The 'Spanish' flu claimed the lives of some 300,000 people (or $6 \%$ of the population) in six weeks, and AIDS has claimed 3.3 million lives in 30 years.

For both good and ill, these pandemics also accelerated processes and trends already apparent but not yet in full swing. For instance, they put urgency into the search for biomedical means to prevent or effectively treat such diseases so as to avoid their recurrence on such a disastrous scale. Thus, vaccination against smallpox was readily adopted by the colonial authorities at the Cape within less than a decade after its discovery in England in 1796. The country's first national public health system was created in 1919 in the wake of the 'Spanish' flu, after it had been under unproductive discussion for several years before this. A polio vaccine was already under development locally in the 1950s when the state-of-the art American vaccine arrived and was successfully employed to all but remove polio as a threat. More recently, anti-retroviral therapy has been extensively rolled out as effective treatment for HIV after nearly a decade of AIDS denialism in the country's highest governmental circles. 
But pandemics have also fuelled existing negative trends and attitudes, most obviously in prompting stigmatisation and furthering racial segregation. In the face of one pandemic after another, authorities argued that urban Africans should be moved to separate locations so as not to infect whites. This attitude produced their forced removal in 1901-1904 to newly-created urban locations, such as Ndabeni in Cape Town, New Brighton in Port Elizabeth, Ginsberg in King William's Town and Klipspruit (which became Soweto) in Johannesburg. Less than two decades later, the official response to the 'Spanish' flu saw even more segregated African locations created: Langa in Cape Town, Batho in Bloemfontein, and Western Native Township in Johannesburg. Such urban African locations became the model for the segregationist and apartheid South African state.

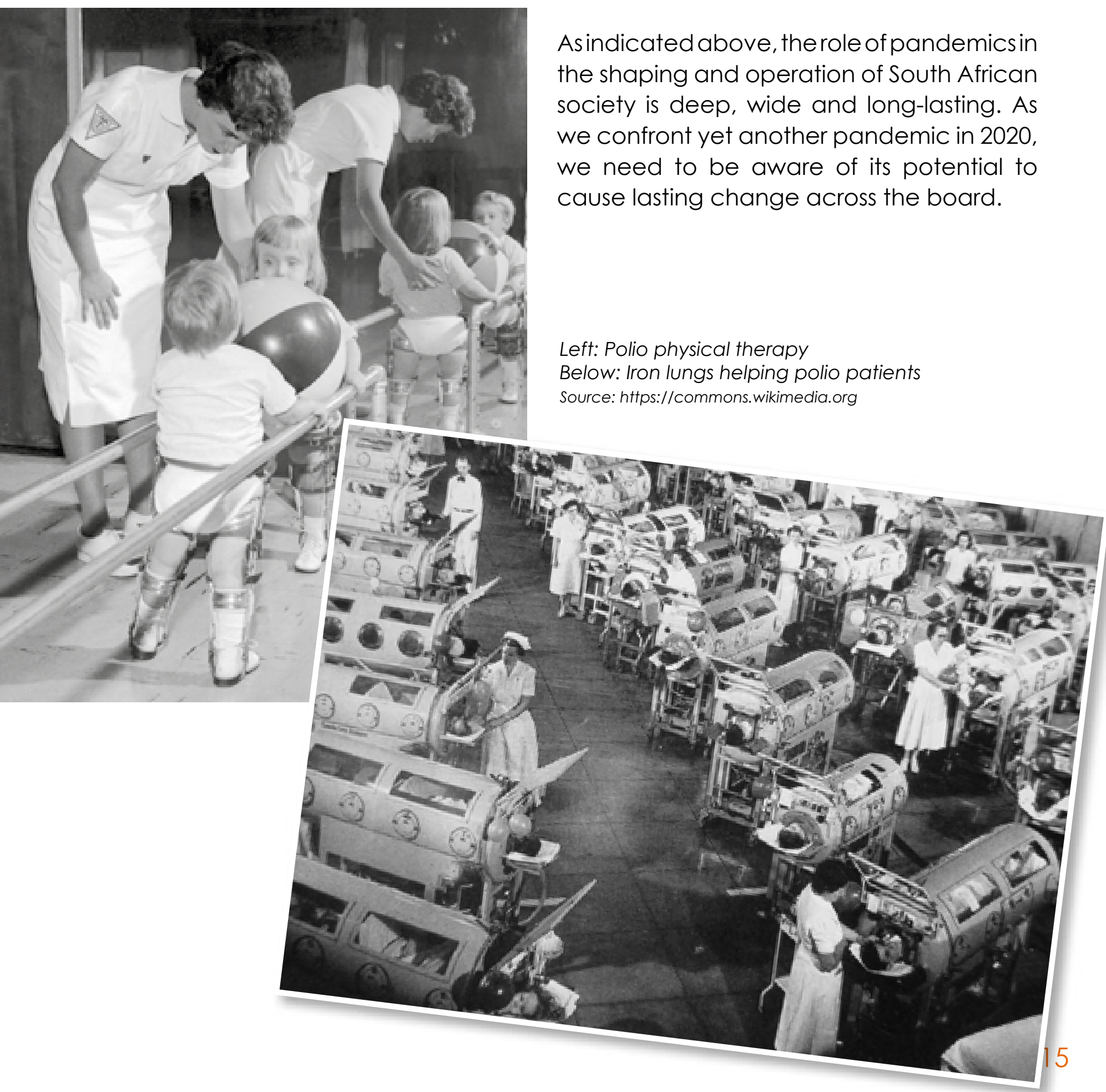




\section{CHAPTER 3}

Infectious Diseases in World History

Viruses and viral infections are probably as old as life is on this planet. There is evidence that our human ancestors were exposed to viruses, but because populations were small and isolated, these were not pandemics as we know them today. The establishment of agricultural communities, animal domestic ation, and much later colonialism caused new viral epidemics and brought us into modern times of vaccine and antibiotic development.

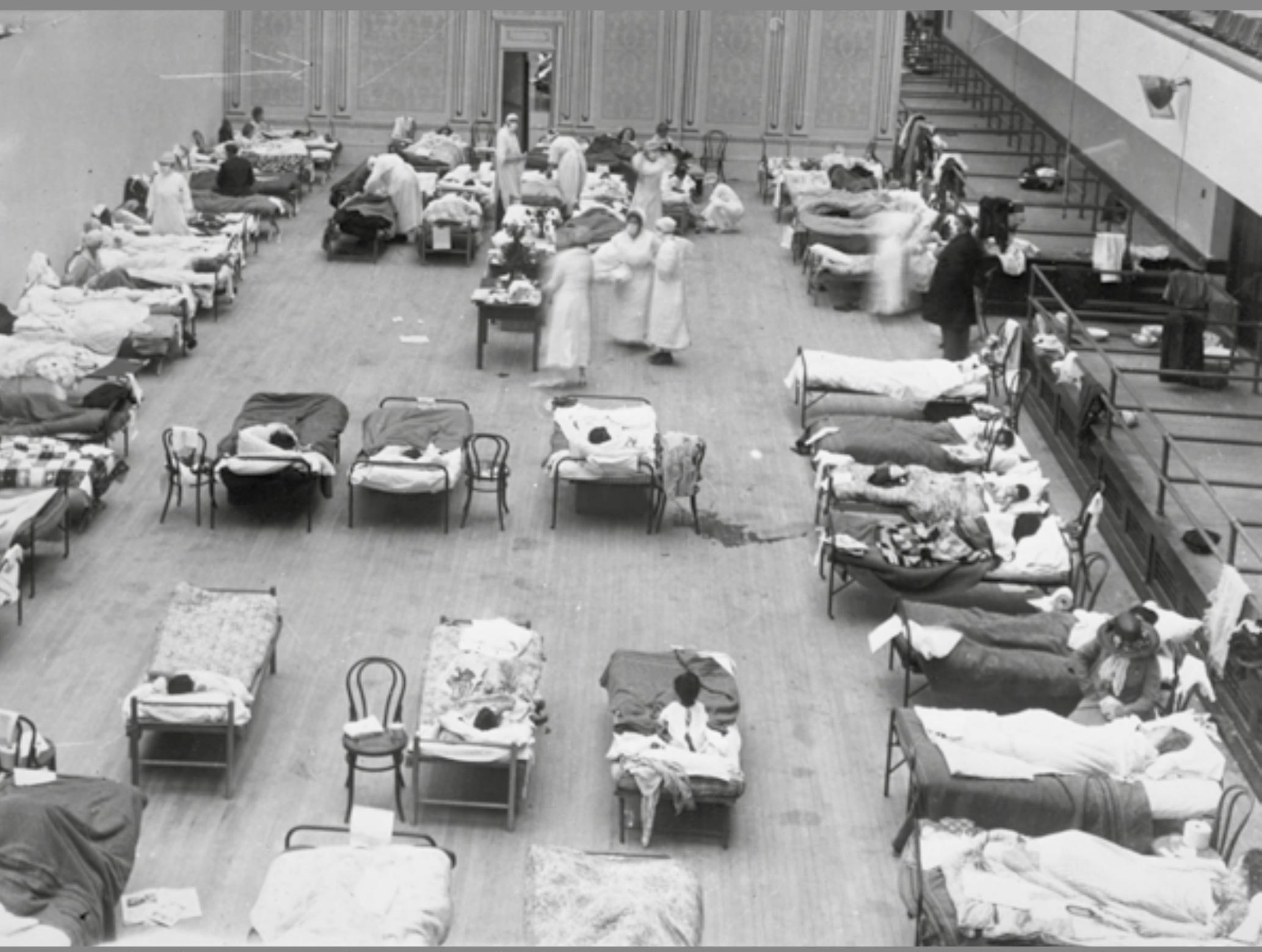

Volunteer nurses from the American Red Cross tending influenza patients in a temporary hospital. Source: https://commons.wikimedia.org 
In Chapter 2, we saw how epidemics have periodically had devastating consequences in South Africa. We now look at epidemics throughout history in a broader perspective, looking further back in history and throughout the world. It is useful to understand how human evolution, history, and pre-history have been shaped by our interaction with viral and bacterial pathogens.

\section{Pre-history}

During our pre-history, say the Neolithic period (this is at the dawn of agriculture), the way viruses interacted with humans was different. Because population sizes were much smaller, there were fewer opportunities for sustained virus transmission. The viruses that did cross over from animal populations (which are generally RNA viruses, like coronaviruses and Ebola-like viruses) could not evolve to sustainable levels because there were simply not enough people to infect. People who study mathematical biology have shown that sustained transmission between humans requires populations greater than a minimal size and with sufficient contacts.

\section{Epidemics and colonialism}

The role of viruses changed as our populations became much bigger and there was differentiation into different countries and nations. Over time these worlds started colliding. For instance, during the colonial era the Americas and Africa were colonised by European nations. The so-called Old World came into contact with the so-called New World, and with the colonists came new diseases. Very often the viruses associated with the Old World were not known in the New World, with the consequence that people in the New World often lacked natural immunities. For example, when the Spanish army

The so-called Old World came into contact with the so-called New World, and with the colonists came new diseases. invaded the Aztecs in the 1500's, a major factor in the Aztecs' defeat was the introduction of smallpox and other infectious diseases by one or two Spanish soldiers. As smallpox spread through the Aztec population, their armies and civilians were largely decimated by this new virus.

\section{Impact of our association with animals}

RNA viruses became more prominent as our population sizes grew and as our agricultural practices changed. A major reason for annual flu epidemics is probably the ability of the influenza virus to replicate in human, bird, pig, and duck hosts, as well as wild birds. This sharing of hosts is also what makes the virus so effective in changing. The genetic material of the influenza virus is segmented. If a strain from a chicken mixes with a strain from a pig, which can happen if two viruses infect the same animal (see the diagram), they may swap genetic segments in a random 
Chicken infected with Influenza subtype A

Pig infected with Influenza subtype B

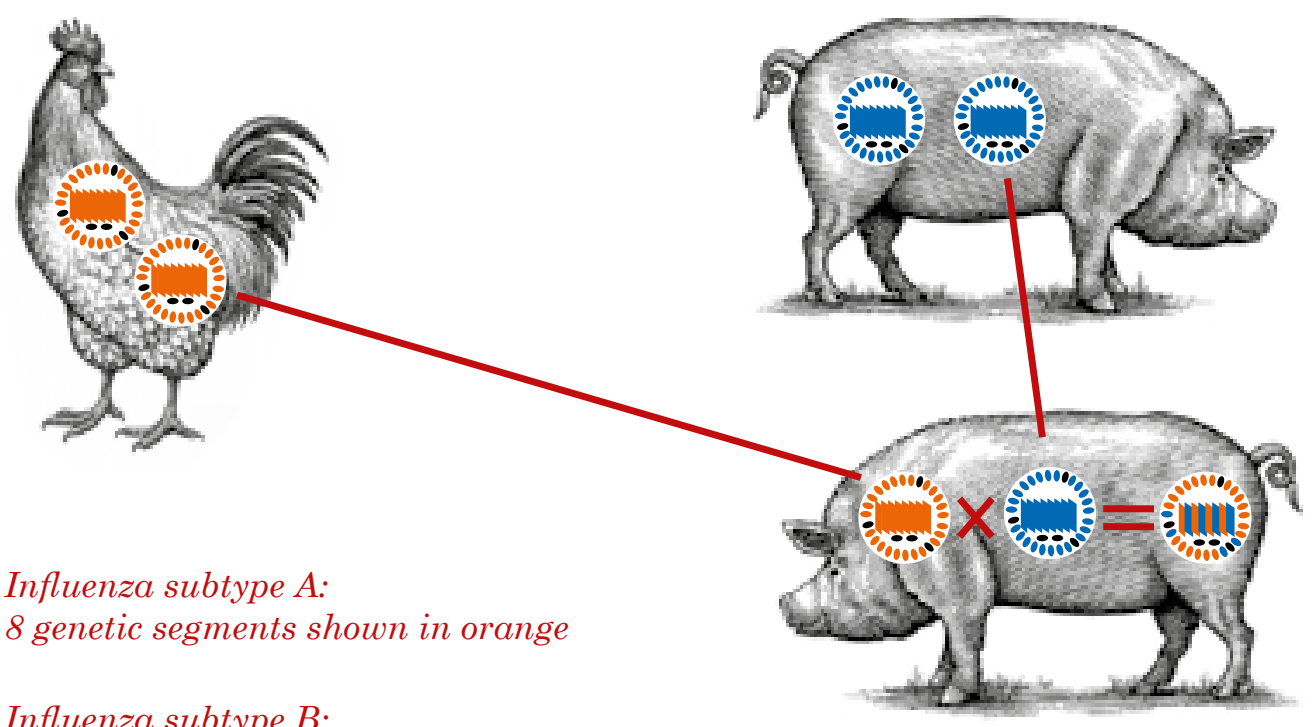

Influenza subtype B:

8 genetic segments shown in blue

Pig infected with Influenza subtype A and subtype B because they are on the same farm and as a result the segmented genomes can

New subtype C:

a mixture of $A$ and $B$ genetic segments reassort producing a new subtype (subtype C)

Figure 3.1 How influenza virus may produce a new subtype by genetic reassortment

Copyright: Steven I. Durbach

process called genetic reassortment (or recombination) to produce new virus subtypes.

While we are currently facing a major coronavirus pandemic, the flu, because of this mechanism of genetic change and our association with farm animals, continues to pose a threat, and in the last hundred years has produced several major epidemics. Most famously, the 'Spanish' flu pandemic, which took place around the time of World War I. This pandemic killed an estimated 50-100 million people (3-5\% of the world's population at that time). The Spanish flu virus probably originated from birds. Although typically these viruses can replicate efficiently in humans, they cannot transmit from person to person unless they undergo a series of mutations in some key genes. The more often a human is infected by a bird-associated flu virus, the more likely the mutations required for a human epidemic will take place. It is a simple 'numbers game'. That is why when flu outbreaks occur in chickens, the entire chicken population must be destroyed to prevent the potentially devastating consequences of the bird flu spreading to humans. More recently, major flu epidemics that killed more than 100000 people occurred in 1957 and 1968 (the Hong Kong flu) in Asia, and worldwide in 2009.

Dramatic examples of how agricultural and food-making practices have brought humans closer to animal populations and thus to novel RNA viruses also abound. 
For example, the Ebola RNA virus comes from humans eating wild animal meat such as monkey meat (called bush meat). The National Institute of Communicable Diseases in Johannesburg was instrumental in tracking these viruses and was the first to discover the source of the Ebola virus about 40 years ago, through work by the famous South African Virologist Margareta Isaacson who tracked the virus down to its source in Zaire in 1976. Ebola has a very high mortality rate, of about $60-70 \%$, and is an excellent example of how a virus can be too lethal for its own good. Unlike the flu, which can infect millions annually, in large part because it does not debilitate its host too much, Ebola makes its host so sick that infected people generally isolate and, therefore, transmit the virus less frequently. Moreover, Ebola relies on contact with bodily fluid for its transmission, which also makes it more difficult to spread than airborne viruses. Nevertheless, one of the most recent Ebola outbreaks in East Africa (2013-2016) claimed more than 11000 lives. This epidemic, however, prompted

When a virus jumps to humans from animals, as in the case of Ebola, the virus is most virulent, but sometimes evolves over time to become less virulent. the development of a novel vaccine. In the wake of this outbreak, the Coalition for Epidemic Preparedness Innovations (CEPI) was formed to foster a coordinated approach to developing new vaccines in order to provide countries with a vehicle to develop and prioritise the most appropriate vaccines to deal with future outbreaks.

Fortunately, viruses often also evolve to become less virulent. This allows a virus to spread to larger numbers of people. Often when a virus jumps to humans from animals, as in the case of Ebola, the virus is most virulent, but sometimes evolves over time to become less virulent.

Ebola periodically jumps to humans from an animal reservoir. Wild animals represent a potentially extraordinarily rich reservoir of different types of RNA viruses that can on the rare occasion cross the species barrier to us. The SARS CoV-2 virus, for example, is hypothesised to have originated in a so-called 'wet market' in China where people trade in wild animals. These wet markets bring humans into close contact with wild animals and increase the probability of successful transfer from an animal to a human host. This probably happens often but the transfer leads nowhere in most cases due to a lack of sufficient human-to-human transmission. Viruses spreading among animals are generally not adapted to human hosts, so they are usually not successful in triggering an epidemic among humans. But the more animal to human transmission occurs, the greater the chance that rare variants with a lucky mutation (because mutations are entirely random) arise that can efficiently replicate in a human host, and, as with SARS CoV-2, have devastating consequences. 


\section{The bubonic plague and other epidemics}

Throughout history, human society has periodically been impacted by massive epidemics leading to the death of a significant proportion of the human population at the time. These include various plagues or epidemics caused by both bacteria and viruses. Above we described the influenza virus, but the range of infectious diseases is enormous. An epidemic can be relatively minor, or it can decimate large swathes of a population, as occurred with the bubonic plague, caused by the bacterium Yersinia pestis. The plague, now easily treatable with antibiotics, probably spread to Europe in the Middle Ages via the expanding trade routes brought about by increased shipping. The disease was spread by rats that were largely immune to the bacterium. Fleas that bit the rats went on to bite people, thus transmitting the bacterium. The earliest known outbreak was in Rome in 590 AD. The most devastating outbreak was in Europe, Asia, and North Africa in 1346-1353. In those seven years, it killed $75-200$ million people, which included $60 \%$ of the population of Europe.

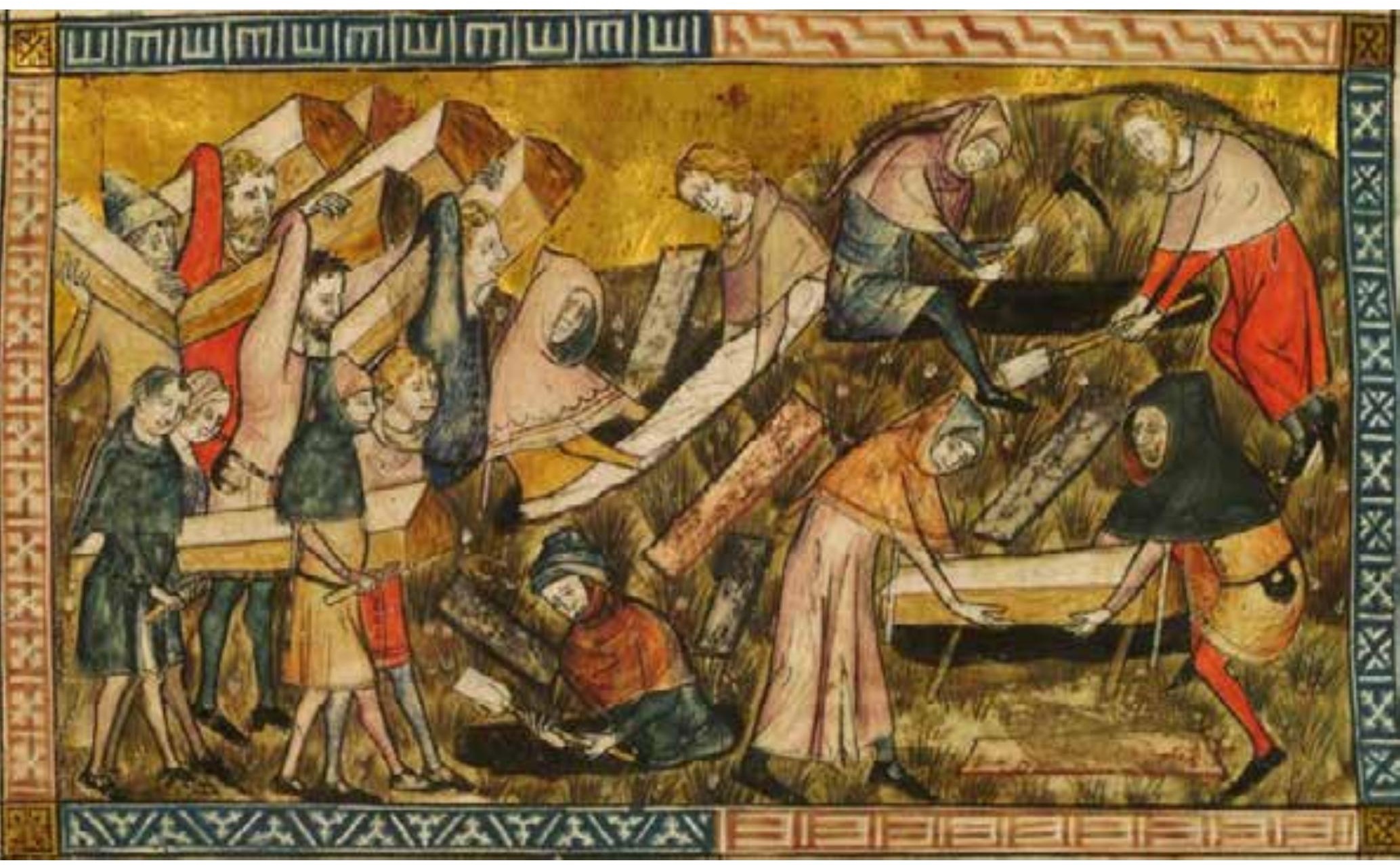

The people of Tournai bury victims of the Black Death. (Tournai, c. 1353).

Source: https://commons.wikimedia.org/wiki/File:Doutielt3.jpg 
It is also interesting to note how human behaviour can affect the re-emergence of certain outbreaks. Measles, for example, was well controlled by vaccination that was developed in the 1950's and was declared to be eliminated in the US by 2000. However, strong opposition to vaccination by concerted misinformation by antivaccination campaigns has led recently to a resurgence of the disease.

\section{History of vaccination}

The biggest breakthrough our species has had against viruses has been with the advent of vaccination. Vaccination has a surprisingly long history, but has been taken up into the Western scientific canon only in the last 220 years. This occurred when Edward Jenner noted that cow maids whose job it was to milk cows would occasionally catch a mild pox disease from cows called cowpox. The women so infected were often relieved as this usually meant they would not catch the dreaded

Survival Function for US Population

For selected Calendar years (1900, 1950, 2000, 2050, 2100)

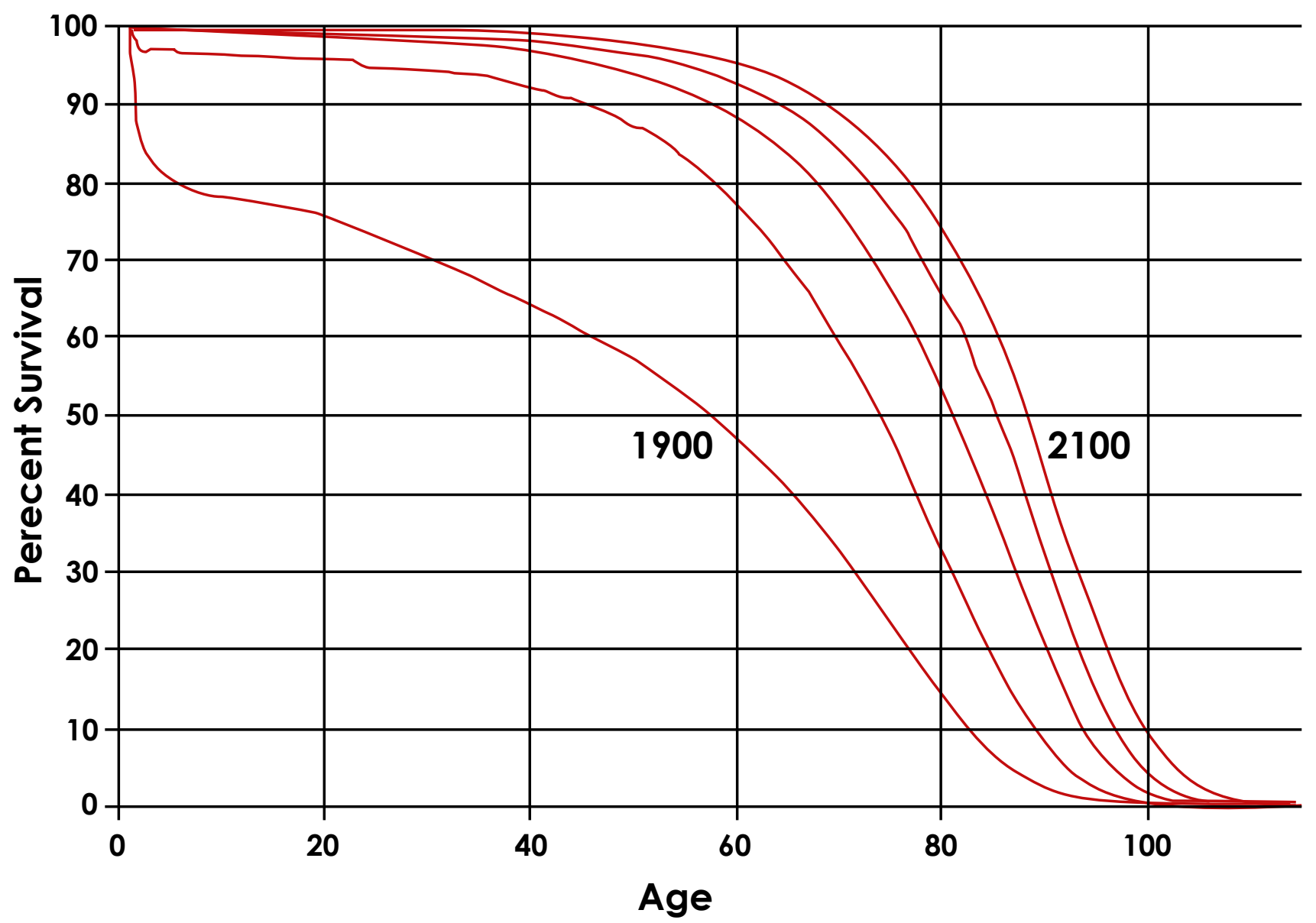

Figure 3.2 Survival Function for US population

Courtesy of US Social Security Administration, https://www.ssa.gov/oact/NOTES/as 120/LifeTables_Body.html 
smallpox disease. Jenner set about taking material from one of these cowpox lesions and inoculated it under the skin of a young boy, James Phipps, whom he later inoculated with material from an actual smallpox lesion. The boy did not go on to get smallpox. For hundreds of years prior to this in China, Africa (for example in the Sudan), and Turkey-and probably elsewhere as well, people had been responding with varying success to smallpox by a process called variolation, where material from lesions of mild smallpox cases was scratched into the skin of potential smallpox cases. In many cases, these people never developed the full-scale smallpox disease.

Although bacteria and viruses are by no means the same, their spread can be similar and their effects can be similar in how they make us sick, because they do so by replicating in our cells. Some bacteria, like the food-borne pathogen Salmonella, replicate in our cells and some like a Staphylococcus, the bacteria responsible for many skin infections, simply replicate in our tissues without invading our cells. Remember that, unlike viruses, bacteria do not depend on a host cell for their replication. Other bacteria, such as Mycobacterium tuberculosis that causes tuberculosis, are similar to the SARS-CoV-2 coronavirus in that they are also transmitted by small droplets produced by coughing (or sneezing). Today we treat bacterial infections with antibiotics. Although antibiotics are in no way like vaccines, they are our main means to combat bacteria. Their impact on human society is about as profound as the impact of vaccines. Antibiotics completely changed the

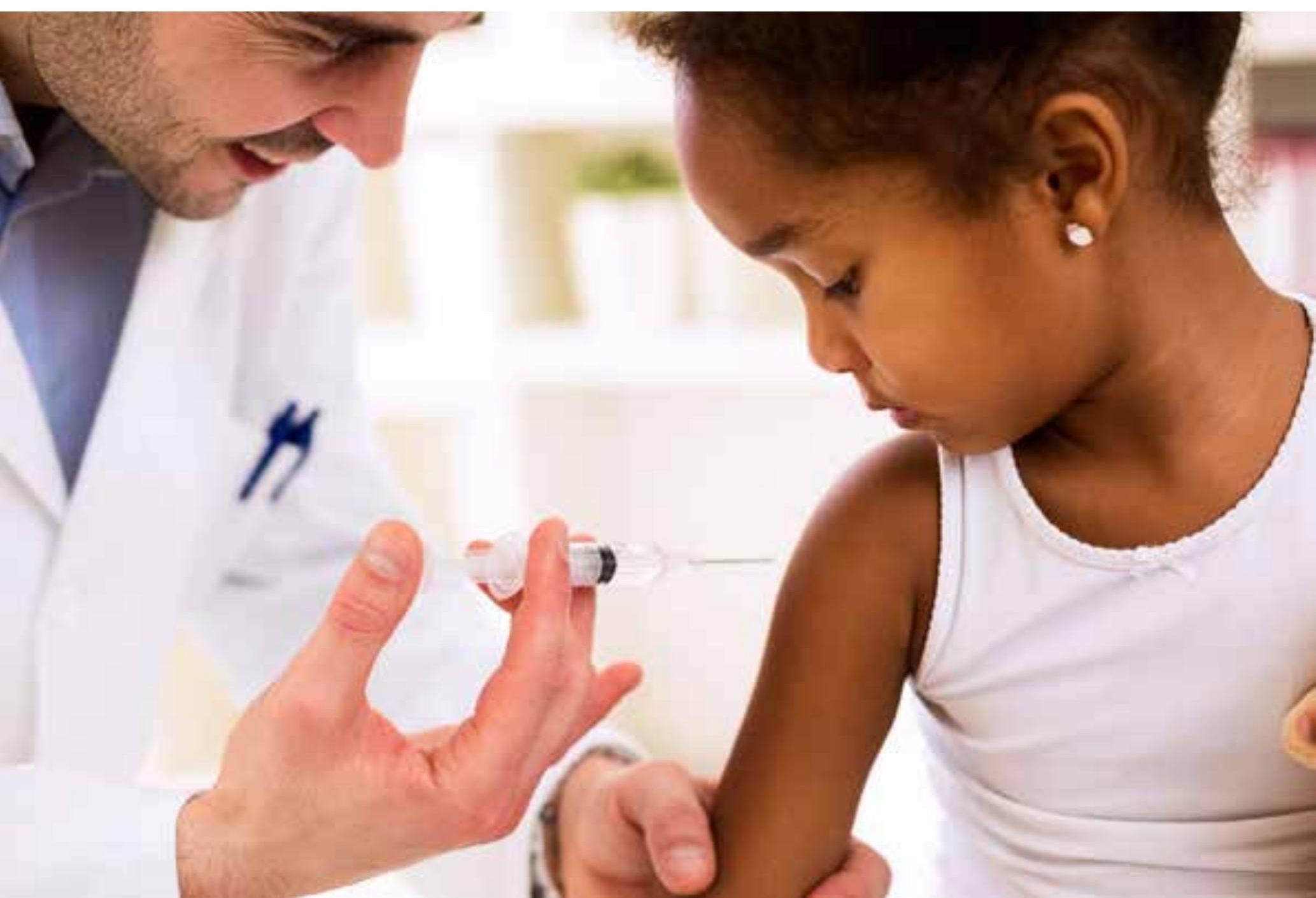




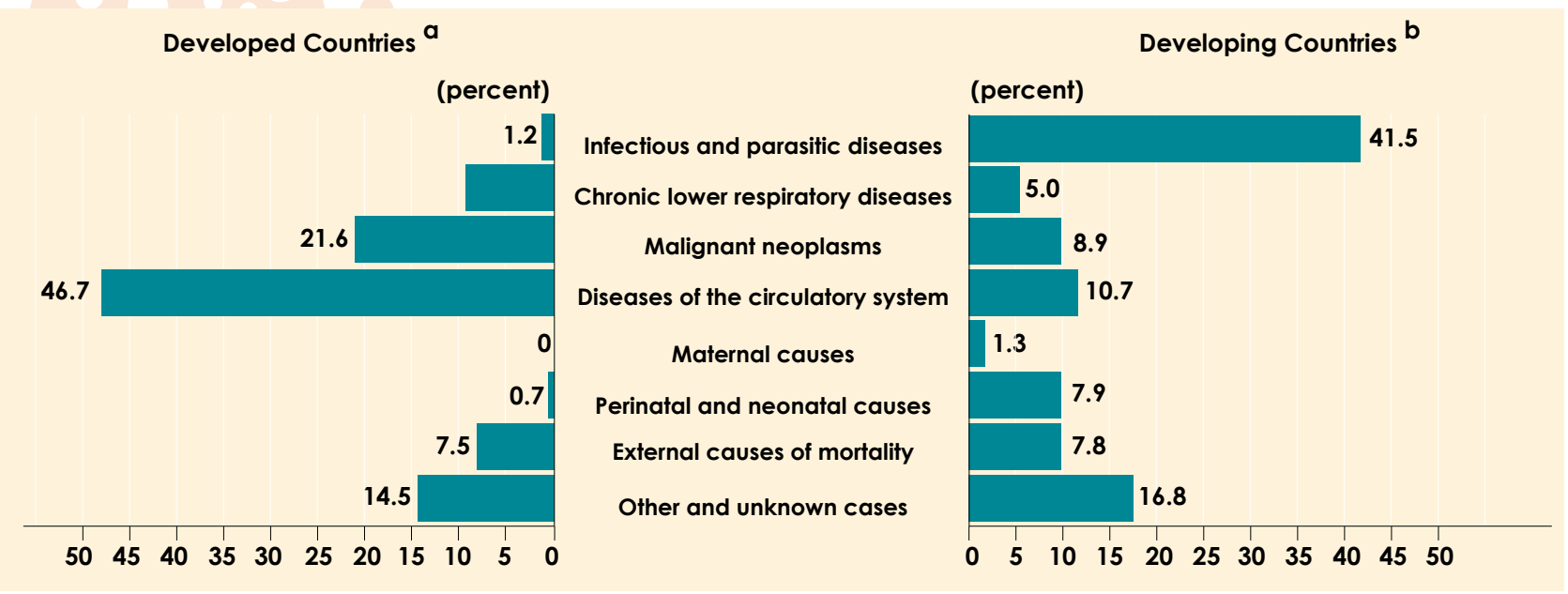

Figure 3.3: Causes of death in developed and developing countries (deaths per thousand) Source: World Health Organisation (WHO) The World Health Report 1995. Bridging the Gaps (WHO, Geneva, 1995)

threat that micro-organisms had on our lives. It certainly made us very confident in our position in the natural world. Now we are not only at the top of the food chain. We are also no longer at the extraordinary mercy of the microbial world.

The US actuarial data in Figure 3.2 show that a little over a century ago, at least in the US, one in five infants did not make it to the age of five, and one out of three did not live beyond 40. The situation, however, has greatly improved. In 1900 the probability of surviving to 50 years old was $50 \%$, whereas in $200050 \%$ are projected to live to well into their 80s. The most impressive feature is the massive improvement in child mortality, which is all but eliminated in children born from the 1950's onwards. This is primarily the result of large-scale vaccination programs. Similar improvements have occurred elsewhere, although the developing world lags behind, or has been partly neglected in this success story.

Because of the huge research and development costs needed to develop new antibiotics and vaccines, pharmaceutical companies often invest little in vaccines and drugs for countries that cannot afford them. Organisations such as the Bill and Melinda Gates Foundation have contributed huge amounts of money for developing drugs for diseases primarily afflicting the Third World. Great progress has been made for diseases such as TB as a consequence of this effort. South Africa has been a great beneficiary, and important research at local universities and institutes has been able to flourish towards this end. But a great disparity still exists if you consider the Figure 3.3 from the WHO. Note that non-infectious diseases are more prevalent in developed countries, but mortality due to infectious diseases is significantly higher in developing countries. 


\section{The discovery and impact of antibiotics}

We briefly look at how antibiotics were first discovered. The environment of the soil where most microbes reside is a very competitive place where diverse and abundant populations of microbes constantly compete for limited resources. Consequently, many of these microbes produce chemical compounds that inhibit the growth-or kill-neighbouring competitor microbes. Some of these compounds can be quite specific, only harming the competitor. These compounds have been isolated by microbiologists and purified or synthesised by chemists. They are known as antibiotics. It is important to remember that antibiotics do not kill or inactivate viruses. They are, however, also useful and often necessary if bacterial super-infection takes place during a viral infection, as is commonly the case in the upper respiratory tract that is, in the nose, sinuses, throat, bronchi, or lung tissue. The medical judgment needed to make such clinical decisions is considerable, and the growing problem of antibiotic resistance is intimately concerned with overuse of antibiotics in poor or 'defensive' medical practice. (Another major cause is the use of antibiotics in farm animals to stimulate their growth.)

About 2,500 years ago, the ancient Egyptians under the Pharaoh Imhotep discovered by trial and error (one component of the scientific method) that wounds healed quicker if covered with mouldy bread! That is because the mould on your bread is often a fungus called Penicillium, which produces the compound we have named penicillin that inhibits the growth of certain bacteria that cause infection. This is the earliest known expression of the antibiotic effect. Alexander Fleming famously (around 1928) noted the petri dishes in which he had grown a culture of bacteria were inhibited by a fungus contaminating his experiment. This is a common and annoying experience for microbiologists. But, importantly, Fleming did not just abandon these dishes. Rather he turned this mess into a serendipitous discovery by asking: what does this mean? He proceeded to find that the contaminating fungus was producing a compound that later on would be purified and called penicillin, which would save millions of lives. Penicillin, for example, prevented devastating outcomes from often quite superficial wounds that, due to the growth of contaminating bacteria in the wounds, could lead to gangrene, requiring amputation of limbs, or sepsis (bacteria in the blood), which is very hard to treat and often leads to death.

Following the discovery of penicillin, many other antibiotics have been discovered for treating a variety of bacterial infections. A major problem today is the overuse, or abuse,

The mould on your bread is often a fungus called Penicillium.

Source: https://commons.wikimedia.org

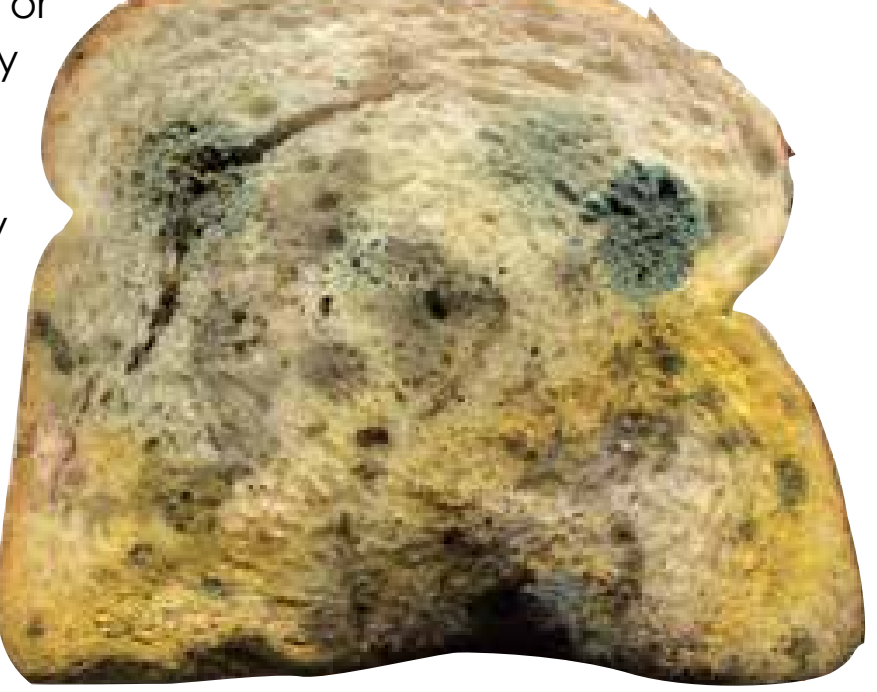



of antibiotics. Recall that the soil where most of these microbes reside is a highly competitive environment. Bacteria on the receiving end of these antibiotics also have their own tricks to subvert the assault from a neighbour's antibiotic. They may contain genes that produce enzymes that inactivate the antibiotic. Bacteria with such genes are said to be antibiotic resistant. This is a classic arms race. The genetic elements conferring antibiotic resistance often reside in plasmids and transposons that can be passed between bacteria. This is Nature's way of speeding up evolution. Genetics, microbiology, and evolutionary science teach us how microbes evolve and adapt to ever-changing environments, and why we should be cautious about the overuse of antibiotics.

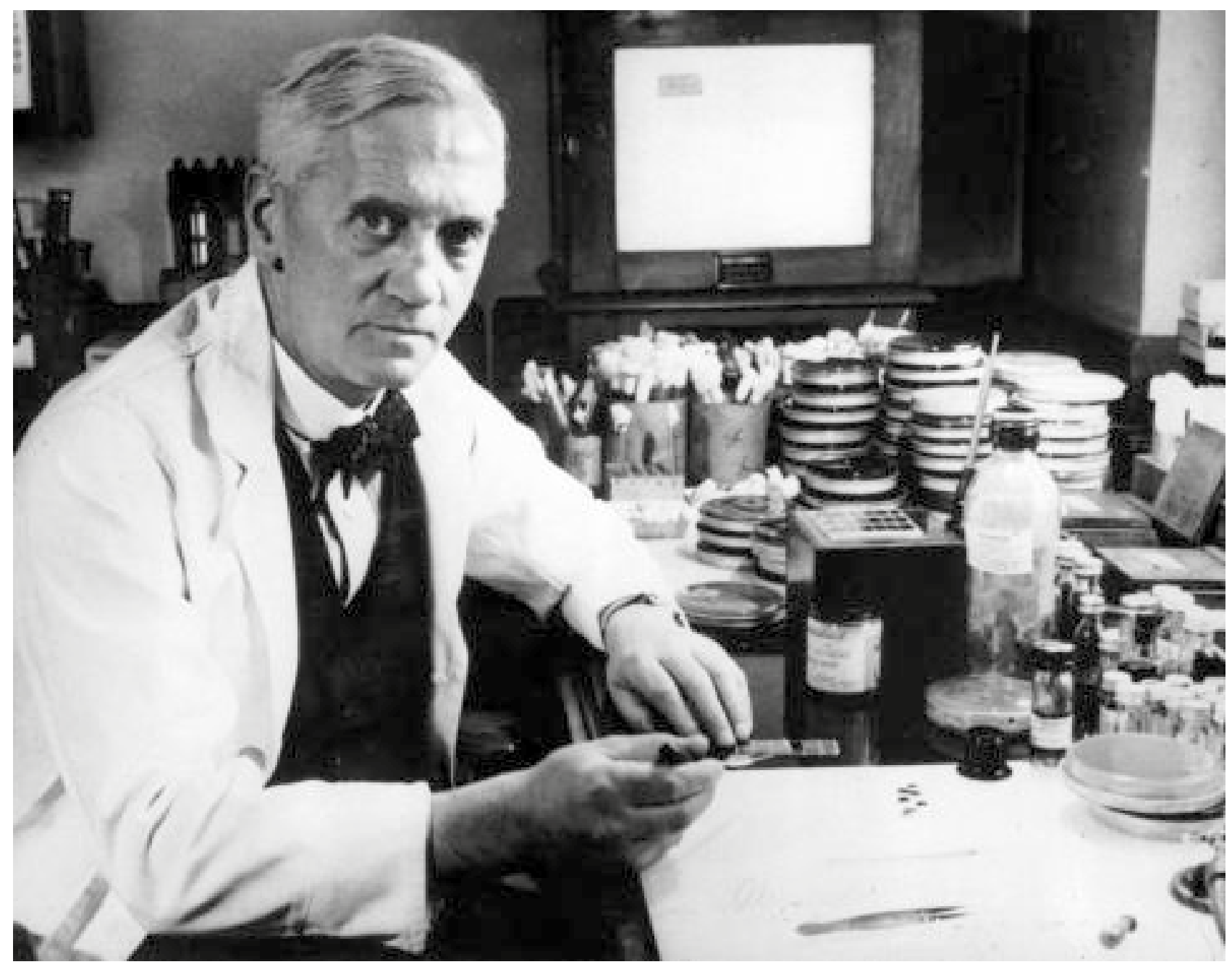

Alexander Fleming

Source: https://commons.wikimedia.org 
CHAPTER 4

Quarantines, Social Distancing, and All That

Covid-19 is an infectious disease, meaning that it is passed from person to person. It is not a disease that arises on its own in a patient. Through a series of questions, we examine how exactly Covid-19 is spread from one person to another and also the various steps that have been taken with various degrees of success to stop its spread. We also look at how some of these steps are not new, but rather have been used throughout human history to stem the spread of other infectious diseases that have affected human populations throughout the ages: the bubonic plague in the 1600 s and the Spanish flu of 1918, to name a few examples. While Covid-19 is new, many of the measures presently being implemented to stop its spread are old and proven techniques.

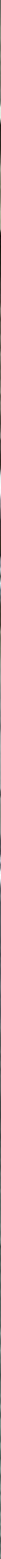




\section{How does the coronavirus spread?}

The coronavirus spreads mainly through droplets or aerosols that an infected person breathes out. We then either breathe these in or acquire them directly through our nose or mouth. Such droplets are deposited on surfaces that we touch and subsequently are transferred to our nose, mouth or eyes. The virus enters our body internally mainly by infecting epithelial cells. These are the type of cells that line the inside of our nose, mouth, throat, airways, lungs, and other passages within our body. The virus attaches itself to such cells by means of its spike protein, and then enters and infects the cell. Once infected, a cell reproduces the virus so that the cell contains many, many new viruses. The infected cell, which has become full of new viral particles, then bursts, and the process repeats itself. In this way, the virus spreads throughout the body, through the blood or lymph stream to many organs. Only when the body's immune system has become trained to fight the virus, by killing it off or preventing its reproduction, does the multiplication of the virus within the body slow down and eventually stop, hopefully before the patient has developed severe symptoms or dies.

\section{How does someone become infected with Covid-19?}

Whenever we breathe, the air entering and leaving our nose and mouth creates small droplets of all sizes (Figure 4.1). The air that we breathe out spreads these droplets and aerosols all over the place. The largest droplets do not go very far. They drop, falling to the ground within a metre or two. But smaller aerosols travel farther and can linger, sometimes floating in the air for hours, before possibly being breathed in by others or landing on surfaces, which, in turn, can be touched.

Although the basic mechanism of how the disease spreads from person to person through

The basic mechanism of how the disease spreads from person to person through droplets and aerosols has been established. droplets and aerosols has been established, many of the details have not fully been worked out. We do not know, for example, exactly how long the virus remains viable in droplets or aerosols and on surfaces. Nor do we know the precise minimum dose needed to become infected. At first the disease was regarded as less infectious than was later established, so the instruction to wear masks was delayed. Now mask-wearing has become compulsory in many countries and guidance on the effectiveness of masks has improved. Good medicine is characterised by a process of learning while discovering new information and summarising the most accurate conclusions from the evidence available at any moment in time. It is, thus, natural that the guidance given by public health authorities should evolve as we learn more and more about this new disease. 


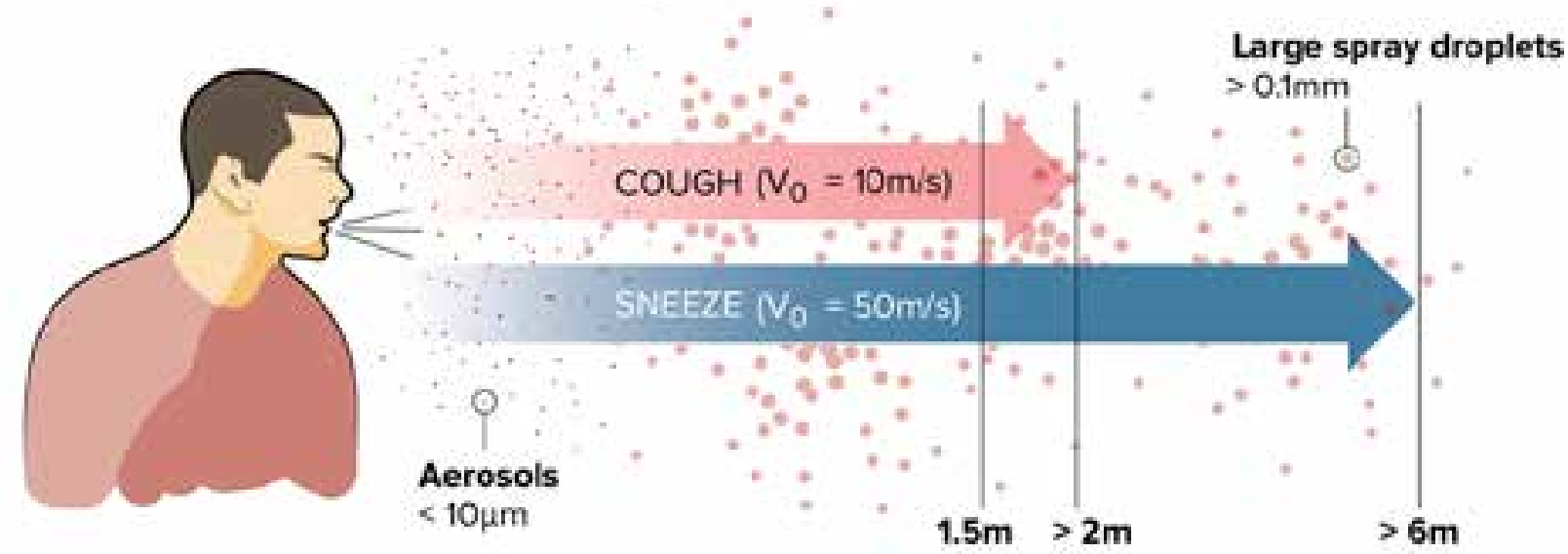

Figure 4.1: Sneezing, coughing, and even speaking gives off droplets of all sizes carrying the virus, thus allowing the virus to pass from one person to another.

A human sneeze gives off droplets of all sizes. After a fraction of a second, the front cloud will have already reached a distance of approximately $75 \mathrm{~cm}$. Many of the droplets are so small (aerosols) that they have hardly fallen. If the person sneezing is infected by Covid-19, someone breathing in these droplets or aerosols can become infected. While the larger droplets fall within a meter, smaller droplets go even farther,and the smallest droplets can linger in the air for an hour or more if there is no wind to carry them away. Even normal speaking or breathing gives off some droplets and especially aerosols of this sort. This is the main way Covid-19 is passed from person to person.

To repeat: Once someone has been infected with the coronavirus, a so-called 'incubation period' lasting from 2-14 days follows before the onset of symptoms. Symptoms may include the following:

- Fever or chills

- Cough

- Shortness of breath or difficulty breathing

- Fatigue

- Muscle or body aches

- Headache

- New loss of taste or smell

- Sore throat

- Congestion or runny nose

- Nausea or vomiting

- Diarrhea 
Each case is slightly different. Each symptomatic person experiences only a few of the above symptoms. This variation in the symptoms exhibited makes it very difficult to diagnose Covid-19 without a test, because many other illnesses present some of the same symptoms listed above.

A patient is said to be infectious when he or she spreads the virus and can, in turn, infect others. A person can be infectious a few days before the onset of symptoms. Consequently, staying away from people who are sick is not enough to stop the spread of the disease. Precautions must be taken to prevent getting infected by apparently healthy people around you, and also to prevent you from spreading the disease to others in case you have been infected but do not yet know it.

We have also learned that some people become infected and consequently are infectious without ever showing any symptoms (asymptomatic cases). They have no way of knowing that they are sick but are able to spread the disease to others.

\section{How to avoid breathing in respiratory droplets or aerosols?}

A combination of the following measures is recommended:

Physical distancing. To the maximum extent possible, try to maintain a distance of at least two metres from other people. This allows the larger respiratory droplets and some of the aerosols to drop to the ground or be blown away by the wind rather than being breathed in or coming into contact with one's skin or clothes. The two metres is a rough guide. Keeping a larger distance would reduce the risk even more. The duration of the contact is also a factor. Coming close to someone else for a short time is a lot less risky than spending a longer time (more than 15 minutes) near someone.

Wearing a mask. It is recommended that people wear surgical masks or triple-layered cloth masks when coming near to people not belonging to their immediate family. The protection provided by surgical masks is limited. The virus may still go through the mask layers but the possibility of this happening is very low.

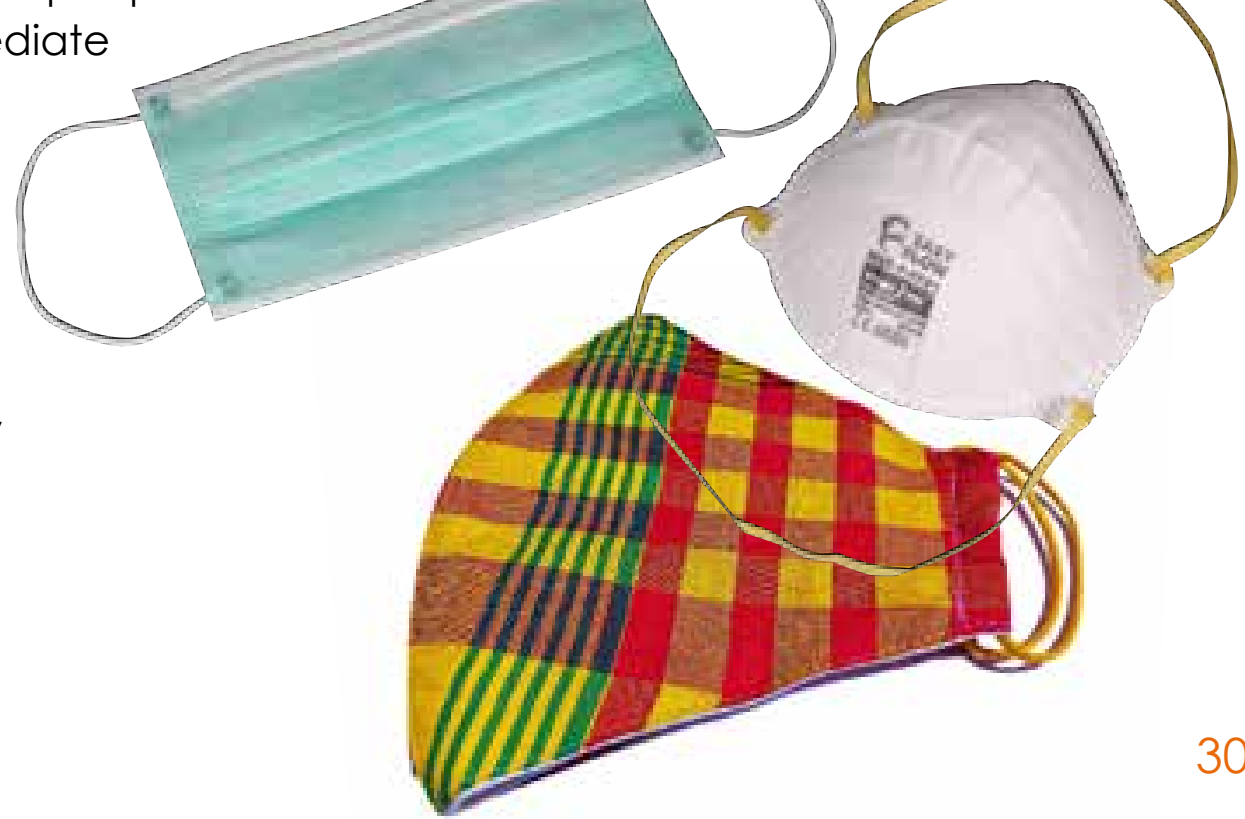




\section{Avoiding places where there are large groups of people, especially indoors.}

Whereas outside the smaller respiratory droplets mentioned above are likely to be carried away by the wind, indoors, especially in places that are not well ventilated, the respiratory droplets can linger and be breathed in.

The above measures do not guarantee that a person will not get infected but greatly reduce their chances of getting infected. The recommendations given by public health experts try to ensure a compromise between avoiding contracting the virus and being able to continue, at least partially, with the usual activities of everyday life.

For medical personnel who work directly with either patients suffering from Covid-19 or with patients who may have Covid-19, more stringent protective measures are recommended. Medical personnel likely to come into contact with Covid-19 patients are recommended to use more tightly fitting so-called N-95 masks as well as goggles and face shields (which additionally prevent introduction of the virus through the eyes). 


\section{How can we destroy the virus on our hands and other surfaces?}

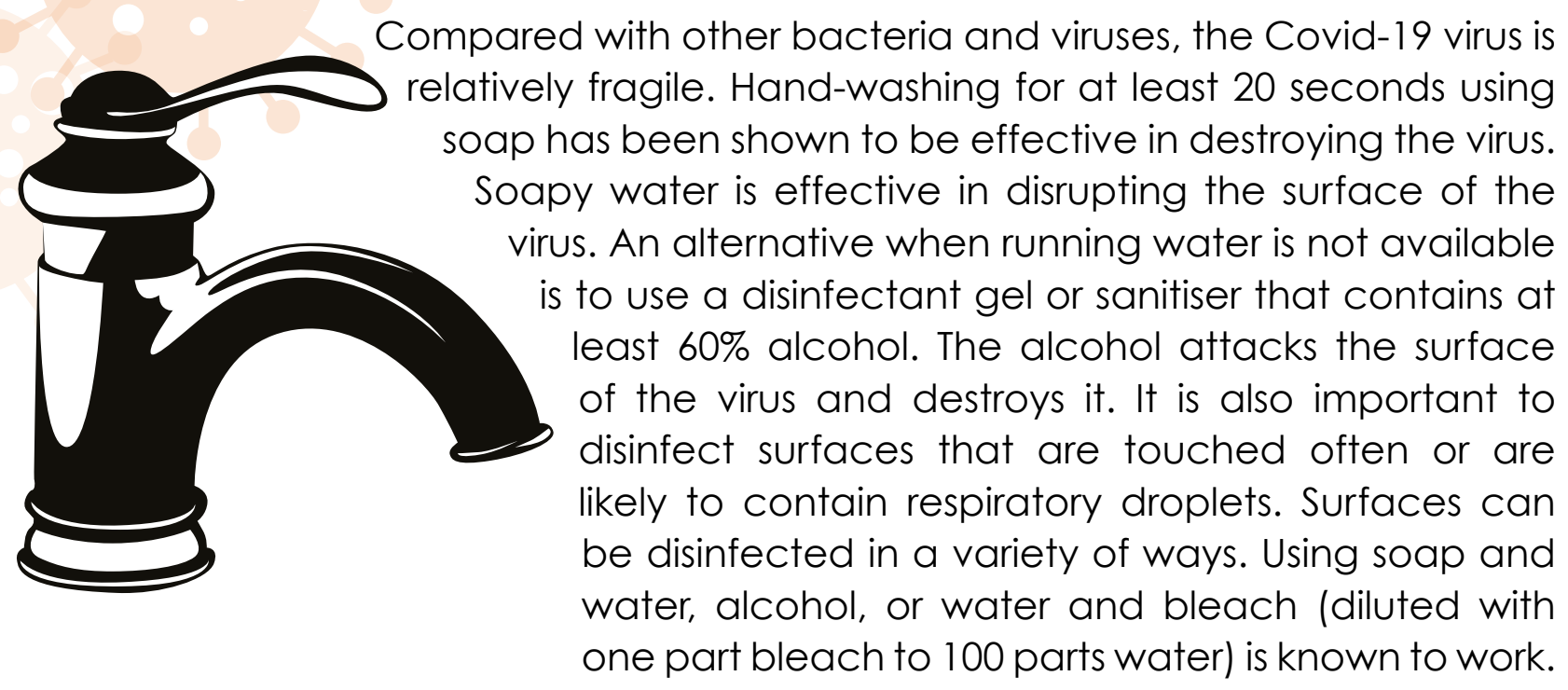

\section{How long can the virus survive on surfaces?}

We can only provide an approximate answer to this question as the lifespan of the virus depends on the type of surface and how effective any disinfecting agent has been in destroying the viruses. Not all viruses are destroyed at the same time but rather gradually. The virus lasts longer on smooth surfaces such as metal or glass than on porous surfaces such as cloth, paper or tissue. The virus can last on surfaces from a few hours to a few days (and perhaps for up to a week). See Chapter 7 for some estimates.

\section{Are quarantines a new idea?}

Wearing masks and isolating ourselves

Communicable diseases date back to the beginnings of human civilization and became increasingly severe as large towns and cities began to develop and people from distant lands started to trade with each other. from others when we show symptoms of Covid-19 is certainly new to us and marks a break with our previous old habits. But the problem of communicable diseases dates back to the beginnings of human civilization and became increasingly severe as large towns and cities began to develop and people from distant lands started to trade with each other. The practice of preventing the spread of communicable disease through isolating those possibly infected is not at all a new idea but dates back to ancient times. In Biblical times, sufferers of leprosy had to live in caves away from people to prevent spread of the disease. The word 'quarantine' derives from the Italian word 'quarantena', meaning a period of 40 days, which was the length of time the crews of ships entering the trading city of Venice had to wait aboard their ships before disembarking and mingling with the Venetians. This simple measure enacted by the Venetians proved 
an effective means of keeping the bubonic plague (already described previously) out of Venice. Similar measures have been reported throughout history from all parts of the world.

While many of the old communicable diseases have been eliminated with modern drugs and vaccines, with Covid-19 we find ourselves having to revert to ancient measures that worked in the past and have also proved effective today when applied consistently.

\section{Are the recommendations for particularly vulnerable populations the same?}

It is recommended that elderly persons and those suffering from certain medical conditions, which increase their risk, adopt stricter physical distancing and other measures. But this is easier said than done. One of the measures adopted has been to limit access to care homes for the elderly, either partially or completely. The idea is to create a Covid-19-free bubble for those most likely to get infected and suffer serious consequences. Many of the major outbreaks have taken place in nursing homes. In many cases, the disease has been brought in by younger staff, who, without

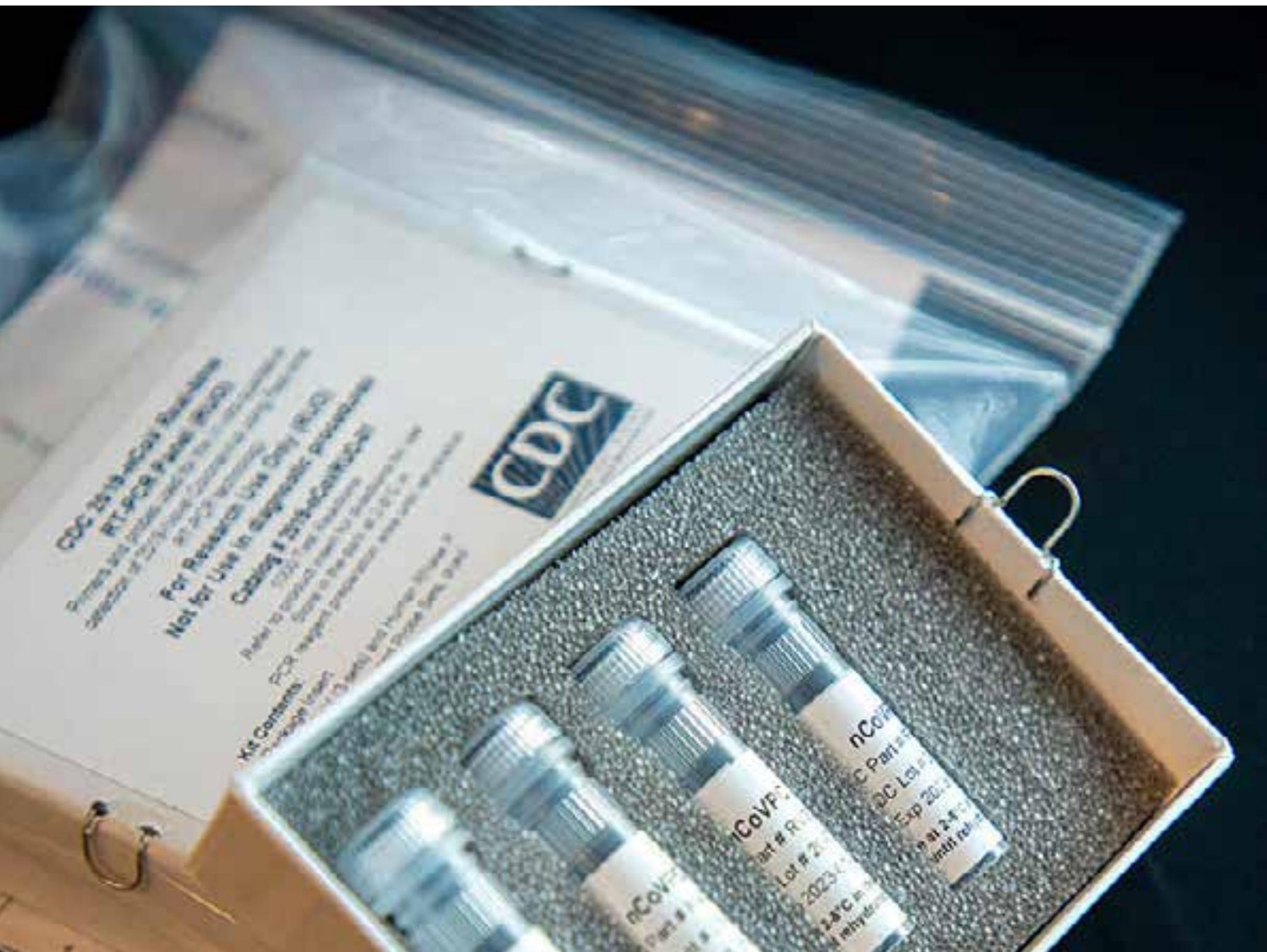


knowing it, have been infected from the outside and introduced the infection into the nursing home. As a consequence, vaccinating the elderly in nursing homes has been identified as a priority.

\section{How does testing help?}

Testing can play a crucial role in reducing, and possibly also reversing, the spread of the epidemic. The idea is to try to identify everyone who is infected and ensure they isolate, thus reducing the spread of the disease. As explained in the next chapter, the number of cases can be made to decrease and even die away, without stopping every transmission event. It suffices to stop the transmission to the extent that, on average, each infected person infects fewer than one other person.

Testing plays a crucial role in a hospital setting, where one infected person can rapidly cause a large number of others to get infected, owing to the large number of contacts and people with weakened immune systems in a hospital setting. For example, in the outbreak at St Augustine's Hospital in Durban during March and April 2020, the infection of one person who was in the emergency department for a short stay led to a chain of infections, which, after passing through many hospital wards, resulted in 119 confirmed infections (39 patients and 80 staff) and 15 deaths. A study done after the incidents highlighted the importance of (1) testing personnel and patients, (2) dividing the hospital into clearly separated zones: a first zone for known Covid-19 patients, a second zone for patients of uncertain Covid-19 status, and a third zone for patients known to be free of Covid-19, and (3) minimising to the maximum extent

\section{The idea is to try to identify everyone who is infected and ensure they isolate, thus reducing the spread of the disease.} possible the movement of medical personnel between these zones, and between the various wards within each zone. If these measures had been implemented, the consequences of this outbreak and similar outbreaks in other hospitals throughout the world would have been less catastrophic.

A good case can be made for frequently testing medical personnel, personnel in nursing homes, and other groups of people coming into contact with more people than average and with vulnerable sectors of the population. Identifying and testing such targeted subpopulations can have a large impact in preventing so-called 'super-spreader' events.

Countries that have been successful in keeping out the epidemic and in controlling the number of local cases have great interest in preventing the infection from being reintroduced by persons entering the country. China, for example, has been successful in reducing the number of local infections to almost zero and requires persons entering China first to quarantine for 14 days and to pass two successive 
RT-PCR' tests. Other countries have implemented similar measures. Of course, if most transmission is local, closing borders has a negligible impact and does little to help.

Most countries routinely test people showing Covid-19 symptoms in order to identify who should self-isolate or be quarantined. One of the problems with Covid-19 is that the symptoms, especially during the initial stage, are non-specific and are easily confused for other common conditions such as the flu.

Ideally, if it were possible to test almost everyone, and also frequently, it would be possible to drive the number of infections to zero. Efforts are underway to massively increase testing capacity, ensure a quick turnaround time, and to develop new tests that can be carried out either at home or locally without having to rely on sophisticated centralised laboratories.

\section{What is the rationale behind stay-at-home, or lockdown, orders?}

At the beginning of the epidemic, many of the countries hardest hit were faced with rapidly increasing numbers and hospitals rapidly filling up to more than they could handle, both in terms of number of beds and intensive care space. To some extent, hospital capacity could be increased, but the situation threatened to overwhelm the health system and cause a large number of deaths. In Italy, for example, it was reported on the news that since hospitals could no longer cope, a decision was taken that no one over 80 years should be brought to the hospital. This meant that such older citizens could not get extra care and would, in most cases, pass away at home. This, of course, placed a heavy emotional burden on medical personnel, who had to sometimes make decisions on whom to admit to hospital and whom to turn away. With the numbers doubling every few days, in many European countries, a decision was made to order everyone to stay at home except for essential workers. These measures largely shut

With the numbers doubling every few days,in many European countries, a decision was made to order everyone to stay at home except for essential workers. down the economies, interfering with people's livelihood despite governmental measures to soften the impact. However, stay-at-home orders proved effective in slowing the spread, and in many countries in bringing the numbers down to manageable levels. In the meantime, testing capacity was increased, and personal protective equipment, initially extremely scarce, became more widely available. In many cases, countries were able to open up again and resume normal economic activity to a large extent. Countries such as South Africa, where medical experts advised the government to put in place an early lockdown, managed to lower 


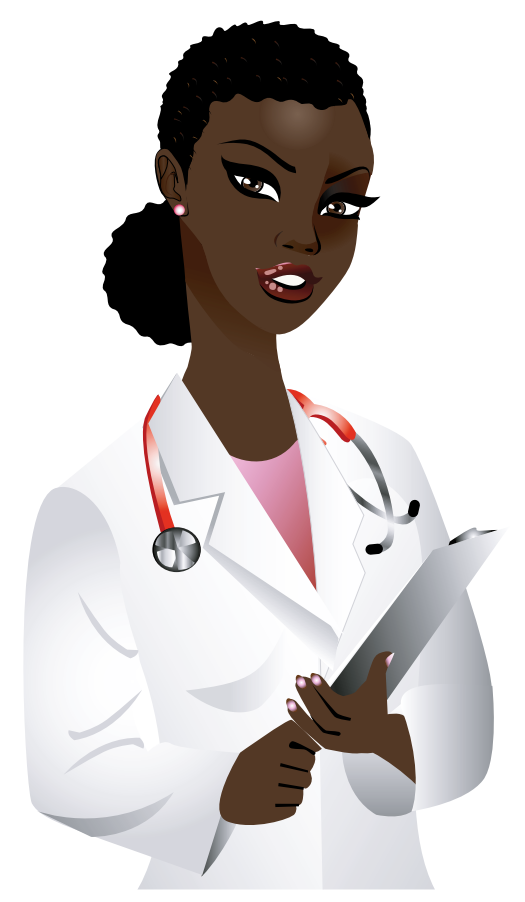

the death rate and to ensure access to hospitals for all citizens. As expected, the rise in infections was not out of control and extra facilities that were set up were not actually required.

\section{What is contact tracing?}

An effective means of stopping the spread of a communicable disease is contact tracing. The idea is to trace, to the extent possible, every contact of each person who turns out to be infected and to encourage-or, in some cases, order those contacts to quarantine or get tested. For Covid-19, the incubation period is believed to be up to 10 to 14 days. This is the length of time a person must self-isolate without having shown symptoms in order to avoid spreading the disease to others. This was how the previous SARS epidemic was successfully stopped.

Contact tracing requires a lot of resources and is extremely challenging when there is a large number of cases. In China, where the number of people infected was first brought down to a manageable level by a long and strict quarantine, aggressive contact tracing has allowed the country to open up and return to nearly normal. In their effort, China has been aided by precise tracking of all its citizens using mobile phone data, so that all contacts could be identified. Other countries have experimented with various mobile phone tracking schemes allowing for contacts to be notified when someone has been infected.

Testing is also an important part of contact tracing, allowing contacts to avoid the disruption of a full 14-day quarantine. Testing with rapid turnaround of results is crucial for avoiding such disruption and securing the cooperation of the potentially infected contacts.

\section{How effective are masks?}

Masks do not provide perfect protection. For a surgical mask, the mask does not seal perfectly around someone's face, allowing some air and-possibly also viral particles-to leak in or out. Nevertheless, videos of persons coughing with and without a mask highlight that substantial protection is provided.

A good reference: WHO, "Transmission of SARS-CoV-2: implications for infection prevention precautions," https://www.who.int/news-room/ commentaries/detail/transmission-of-sars-cov-2-implications-for-infectionprevention-precautions. 
For medical personnel, tighter fitting and better filtering masks called respirators are used. These so-called N-95 masks are certified to filter out at least $95 \%$ of particles 0.3 microns in diameter. (One micron is a tiny unit of length, equal to one thousandth of a millimetre. A human hair is about 20-150 microns thick. A number of viruses are smaller than 0.3 micron such as HIV and SARS-CoV-2 which are about 0.1 micron.) Medical personnel also wear face shields, goggles, and disposable gowns for extra protection. 


\section{CHAPTER 5}

\section{Dynamics of Epidemics}

The spread of infectious diseases among populations can be described using mathematical modelling. Mathematical models play an important role in predicting the future spread of infectious diseases and the impact of various competing policy options. Such models are important for preparing the medical response, including foreseeing how many hospital beds will be needed as well as how many supplies to procure, to cite a couple of examples. 
- Mathematical modelling is imperfect, given limitations in the available data and its reliability as well as uncertainty as to the precise details of how the disease spreads. Human behaviour and psychology as well as messaging by public health officials, governmental authorities, and the media also play an important role. Nevertheless, such modelling provides insight through presenting a range of possibilities for future evolution of the epidemic.

- We first explain the notion of exponential growth. When the number of people infected is growing exponentially, the number of new infections increases by a constant factor after a same time period-say a week. This means that the number of people infected during the previous week always gets multiplied by the same number. When there is exponential growth, a tiny number of initial infections can lead to a huge number of infections rather quickly. This happens when each infected person infects more than one other person. This number is known as R or $R_{0}$ when the entire population is susceptible.

- Public health measures, such as social distancing, can decrease $R$, and if $R$ becomes less than one, the number of infected people will decrease rather than grow, as we have seen at various times.

- However, as people tire of the sacrifices required to keep $R$ below one, second, third, and higher waves of the epidemic may follow. We have seen this already in South Africa and abroad.

- Exponential growth can also be stemmed through vaccination or herd immunity. When enough people have already been infected, contacts that in a fully susceptible population would have led to growth are insufficient for each infected person to infect at least one other person on average. The same protection can be given by vaccines, as we have recently seen.

- A serious worry now is the impact of new mutated strains of the virus, some of which have been seen to spread more vigorously and also to evade, at least partially, some of the present vaccines. It is of great importance to put an end to this pandemic before more dangerous new strains emerge. 
In this chapter we talk about how a contagious disease like Covid-19 spreads through a population. Whereas earlier we focused on the details of transmission-that is, exactly how the disease spreads from person to person, here we shall look mainly at the big picture: how the numbers of people infected change with time. In doing so, we will use some simple mathematics. This mathematics is also applicable to other contexts, such as finance, banking, and engineering. Of course, researchers have found other more accurate ways to model diseases than the one we consider here. Understanding how the disease spreads through a population is very important for planning and for figuring out what steps can best stop or slow down the spread of the disease. It can also help us better understand how certain actions, such as wearing masks and social distancing, beyond protecting ourselves, also serve to protect others and to promote the common good.

\section{Daily new confirmed COVID-19 cases}

Shown is the rolling 7-day average. The number of confirmed cases is lower than the number of actual cases, the main reason for that is limited testing.

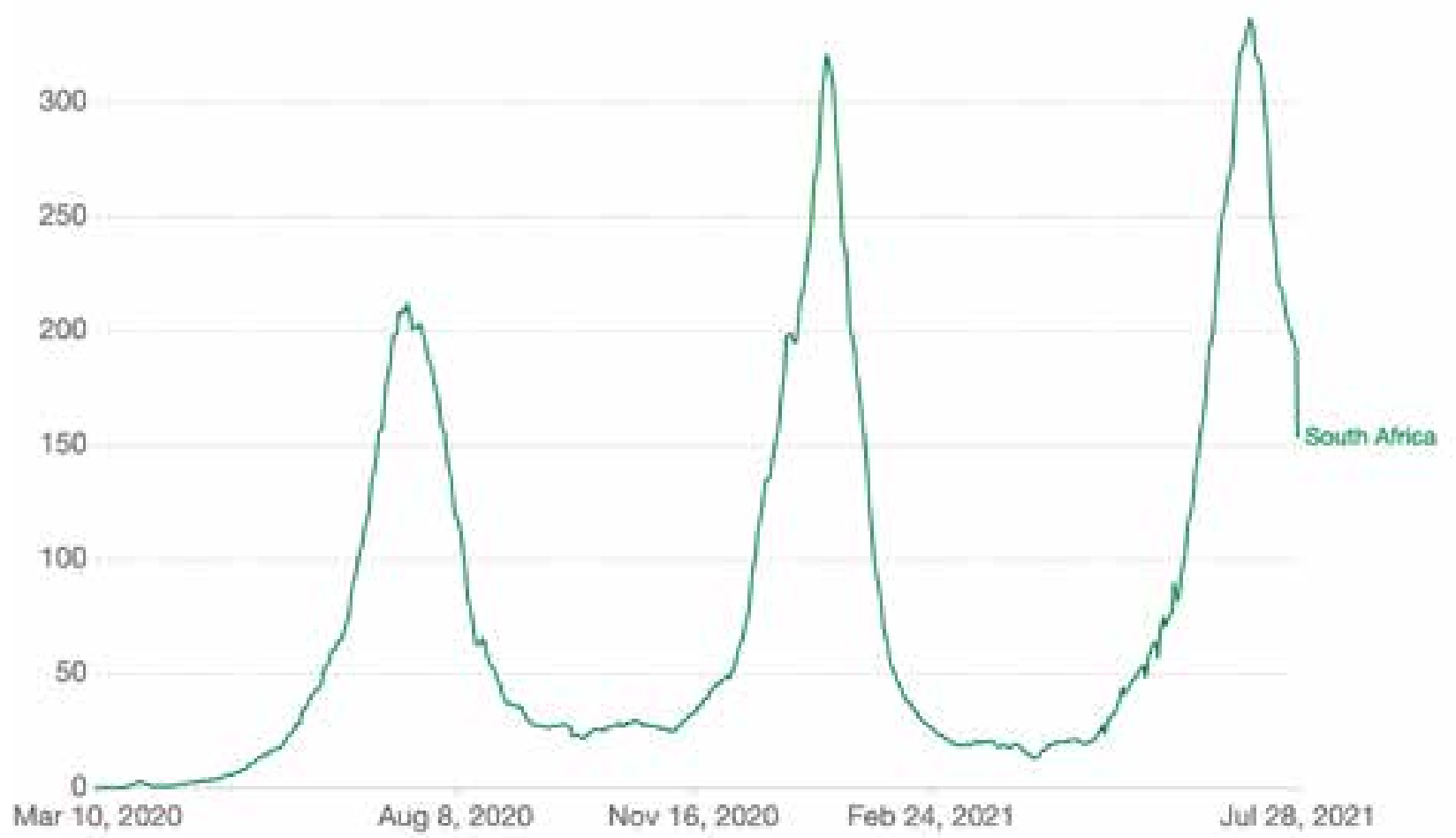

Figure 5.1: Recorded daily number of new Covid-19 cases in South Africa Source: https://ourworldindata.org/covid-cases. Johns Hopkins University CSSE COVID-19 Data. 
We begin by looking at exponential growth, which serves as an accurate model of how the numbers change over the short term, and possibly also the medium term. Exponential growth, however, cannot continue forever. If the numbers get too large, eventually other factors come into play and take over, as we later explain.

\section{What is exponential growth?}

Sometimes a gossip story is said to have 'gone viral' on social media. This means that such a story is spreading very fast, like a virus. We read in the newspapers that the virus is "growing exponentially." What exactly does this mean?

Problem: Let us begin with a simple exercise. Let us examine the three sequences $A, B$ and $C$ of numbers and try to figure out the rule.

\section{Sequence A:}

$1,2,4,8,16,32,64,128,256,512,1024,2048,4096,8192,16384,32768,65536$, 131072,262 144, 524288,1048 576, 2097 152, $4194304,8388608,16777216$, $33554432,67108864,134217728,268435456,536870912,1073741824$, $2147483648,4294967296,8589934592, \ldots$

\section{Sequence B:}

$1,3,9,27,81,243,729,2187,6561,19683,59049,177147,531441,1594323$, $4782969,14348907,43046721,129140163,387420489,1162261467$, $3486784401,10460353203, \ldots$

\section{Sequence C:}

$1,4,16,64,256,1024,4096,16384,65536,262144,1048576,4194304$, $16777216,67108864,268435456,1073741824,4294967296, \ldots$

We have continued each sequence up to 10 billion, but these sequences could go on forever. The trailing three dots ... mean 'and so forth'.

Answer: For Sequence A, each number is equal to two times the number before itself. For sequence $B$, each number is equal to three times the number before itself. For sequence $C$, each number is equal to four times the number before itself.

These are special cases of a geometric sequence. A geometric sequence follows the pattern that every number is multiplied by the same fixed number to get the next number. We may also say that the ratio between every number and the number before it stays the same or is constant. 
Another way to say this is that the numbers obey the rule:

(Next number) $=$ (Constant factor) $\times$ (Current number)

The factor is exactly the same for each number to follow; hence we call it a constant or the common ratio.

The growth factor does not have to be a whole number. It could be any real number greater than zero. Also, the first element does not have to be one but could be any positive number. For example, the following is a geometric sequence, even though it is not so obvious.

$2.73,3.00,3.30,3.63,3.99,4.39,4.83,5.31,5.85,6.43, \ldots$

To confirm that it is geometric, or not, we would have to calculate the ratios of successive numbers to show that they are all equal, or perhaps not.

Exercise: Which of the following are geometric sequences?

\section{Sequence $X:$}

$11.63,23.63,29.03,28.71,33.07,51.04,77.81,96.33,93.90$

\section{Sequence Y:}

$11.96,23.93,35.89,47.86,59.83,71.79,83.76,95.72,107.69$

You may wish to use a calculator to solve this problem.

We can use this type of mathematical method to understand the rates of infection or growth of the Covid-19 pandemic. Let us look at the table of data below of the number of people infected with Covid-19 in South Africa, in which each row is separated by one week, and see how well the data are described by a geometric pattern:

Table 5.1 Growth in Covid-19 cases in South Africa in June and July 2020

\begin{tabular}{|l|l|l|l|}
\hline Week & Date & Cumulative number of cases & Current week/Previous week ratio \\
\hline 0 & 6 June & 45973 & ------- \\
\hline 1 & 13 June & 65736 & 1.43 \\
\hline 2 & 20 June & 92681 & 1.41 \\
\hline 3 & 27 June & 131800 & 1.42 \\
\hline 4 & 4 July & 187977 & 1.43 \\
\hline 5 & 11 July & 264184 & 1.41 \\
\hline 6 & 18 July & 350879 & 1.33 \\
\hline 7 & 25 July & 434200 & 1.24 \\
\hline
\end{tabular}


We observe that from Week 0 through Week 5 the growth from one week to the next is approximately 0.42 , or in other words about $42 \%$ (on average). We say that the rate behaves like a plateau because it is almost constant in time. However, from Week 5 through Week 7 the average weekly growth rate is $28 \%$, with evidence of a gradual drop in the rate.

How long might the epidemic last based on this data? We do not have enough information to make an accurate estimate. There is no reason to believe that the present growth rates will continue into the future unchanged, and the data does not indicate a constant growth rate but a dropping trend from a plateau. Nevertheless, there is great value in making rough estimates as long as one keeps in mind that these estimates are only approximate, and, moreover, that future events might possibly suggest that a new trend is forming. In other words, the predictions could be completely wrong.

Nevertheless, let us proceed with a certain amount of courage. Obviously, the total number of people infected cannot exceed the total population of South Africa, which is presently 58 million. Consequently, let us assume very roughly that the epidemic continues to grow at the present rate until everyone has become infected. (We shall see that the endgame will be less abrupt, but let us for the moment ignore this complication.)

Consequently, from 6 June 2020 (with $4.6 \times 10^{4}$ infections) to when everyone has become infected (5.8x $10^{7}$ infections) requires growth in the number of infected people by some factor. How do we determine this? We estimate this by dividing the total possible number of infections ( 58 million people in the population) by the number of people infected at 6 June

$\frac{5.8 \times 10^{7}}{4.6 \times 10^{4}}=1,260869 \times 10^{3}$

rounded off to 1261.

The monthly growth measured above by a factor of 1.42 per week amounts to $1.42^{4}$, which is approximately equal to 4 per month. To find the number of months needed for everyone in South Africa to get infected, we must solve the equation

$4^{x}=1261$

where $\mathrm{x}$ is the number of months needed for everyone to be infected and the epidemic ends. $4^{x}$ means 4 multiplied by itself $x$ times, with fractional power defined using nth roots. 
One way to solve this equation is by guessing with the help of a calculator.

We find that $4^{5}=1024$ which is slightly too small and $4^{6}=4096$ which is way too big. Consequently, $x$ lies between 5 and 6 . The correct estimate should be close to 5 , so after trying values like 5.25,5.2, and then 5.15, we could settle on $x=5.15$.

For readers in grade 12, the method of logarithms can give the answer quicker. We use the natural logarithm with base $e$, which is the Euler number as opposed to base 10. Taking the natural logarithm of both sides, we obtain

$$
\ln \left(4^{x}\right)=x \ln (4)=\ln (1261)
$$

or

$$
x=\frac{\ln (1261)}{\ln (4)}=5.15
$$

So, according to this estimate the epidemic should end by the beginning of 2021 after about 5 months. We may get an idea of the possible error by using the lowest growth rate where 4 is replaced by $1.24^{4}=2.36$. In this case we would get $x=\ln (1261) / \ln (2.36)$ which is 7.93 or roughly 8 months. This means that the end would be estimated for around mid-2021 instead. But if the growth rate in the future drops further, the epidemic would last longer.

The general rule for a geometric sequence is given by:

$$
F_{n}=A x^{n}
$$

where $n=0,1,2, \ldots$ is an integer. Such a sequence may be plotted using dots, as follows.
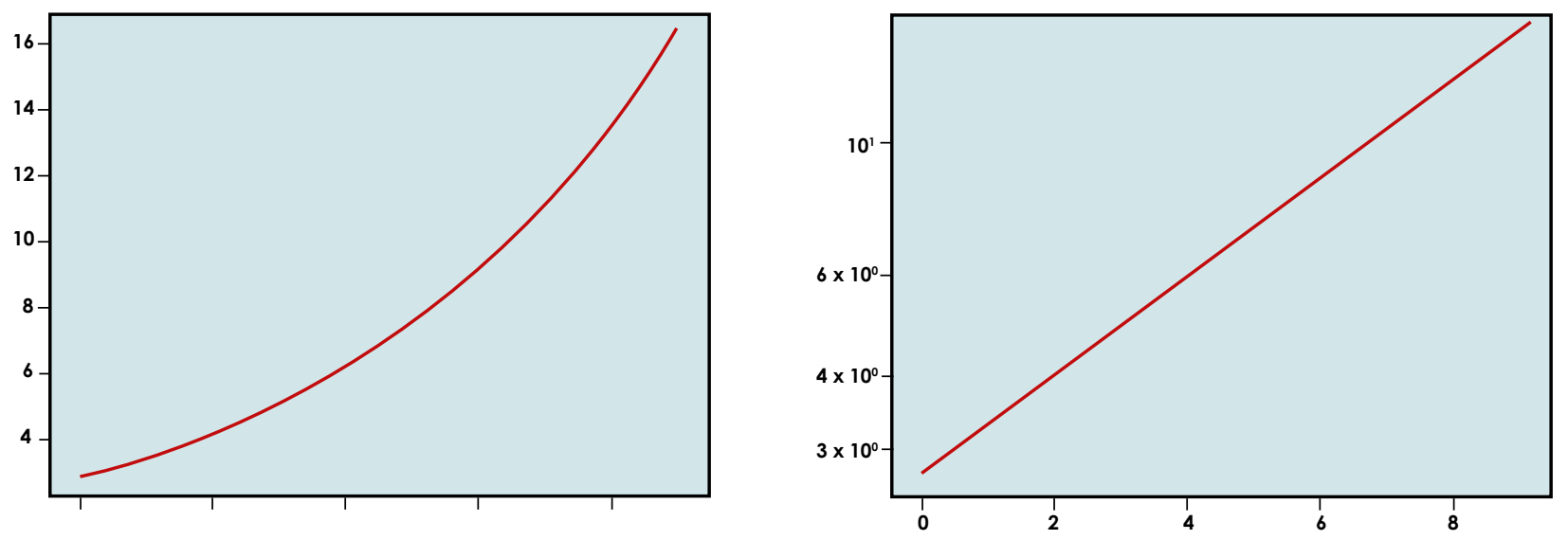

Figure 5.2: Plotting geometric sequences

A geometric sequence is plotted on the left using a linear scale for the vertical axis, and on the right using a logarithmic scale instead. With the logarithmic scale, the dots arrange themselves perfectly along a straight line, showing visually that the growth is geometric. 
The mathematics of exponential growth and decay appears in a wide variety of diverse applications such as radioactive decay, the absorption of light, nuclear chain reactions, finance and banking. In the simplest model of inflation (with a constant rate of inflation), prices grow with time according to an exponential curve.

Because inflation growth is an exponential function, we sometimes find some countries have rates of inflation in the thousands of percent whereas other countries, such as South Africa, keep the inflation rate down to single digit numbers through a strict monetary policy. Money in a savings bank account also grows exponentially, provided that the rate of interest does not vary.

\section{Why (and when) do we expect case numbers to follow a geometric progression?}

Let us consider a simple model of how the disease spreads. Suppose that each person who gets infected in turn, on average, infects the number $R_{0}$ of other people, after a time T, known as the 'serial interval'. As long as not too many people have been infected, with each successive generation, $R_{0}$ as many people are infected as had become infected at a time earlier by $\mathrm{T}$. We know that the virus spreads from person to person by respiratory droplets and aerosols. These are exhaled, to some extent by simply breathing, but even more so by speaking, singing, panting as the result of strenuous exercise, or coughing. These droplets may be directly inhaled by someone nearby, or the small aerosols may linger in the air for hours (especially in an enclosed, poorly ventilated area) and then inhaled by someone. The serial interval $T$ is more a property of the infection itself, but $R_{0}$ is strongly dependent on people's behaviour. If people stay at home and hardly go out, $R_{0}$ will be greatly reduced, and if $R_{0}$ falls below 1 for long enough, the disease will eventually die out and become extinct, at least in the geographical area where that is the case. Wearing masks will also reduce $\mathrm{R}_{0^{\prime}}$ as will avoiding events where many people gather. However, in places like South Africa, where people living

\section{Covid-19 in the United States}

The pandemic struck the United States after Europe. But despite this late start, the United States now leads the world both in cases and deaths. Although the US claims only $4.2 \%$ of the world's population, as of 22 July $2021,26 \%$ of the recorded Covid-19 cases and $18 \%$ of the recorded Covid-19 deaths were from the US. The epidemic first hit the large cities, in particular the New York City metropolitan area, and then spread across the country to less populated states and rural areas. Although the US government shut borders early on, little else was done to prepare, particularly by the federal government. President Donald Trump claimed persistently that the epidemic was a "hoax" and would simply "go away" by itself. When numbers exploded, hospital emergency department and intensive care unit numbers exceeded capacity in many big cities. Moreover, medical personnel lacked needed personal protective equipment and testing supplies.

(continued on page 46) 
in close proximity in informal settlements and traveling using public transport increase opportunities for close contact, some of the preventative recommendations may not be implemented.

In our estimates of the future course of the epidemic based on geometric growth, we assumed that $R_{0}$ stays constant. But from the above discussion we see that we, as individuals, may control $R_{0}$ by adjusting our behaviour, at least to some extent. The government may also affect $R_{0}$ by suggesting guidelines for people's behaviour aimed at slowing, and also possibly reversing, the growth of the epidemic, or by imposing regulations. Identifying infected people and isolating them until they are well are other measures that help stop the spread of the disease. People's perception of the seriousness of the disease and of their own probability of getting infected also plays a role in determining behaviour, and thus the media and also the statements of political and community leaders can make a difference.

Consequently, we see that the spread rate following a geometric sequence assumes that people do not change their behaviour. Another factor affecting the spread is how many people have already been infected. It is generally assumed that when a person is infected and recovers, they retain an acquired immunity for some time, possibly for the rest of their life, as is the case for other types of viruses, although the situation for Covid-19 is uncertain. Consequently, when it is no longer the case that almost everyone is susceptible (in other words, they can be infected), the rate of growth slows down, even if people do not change their behaviour. If a fraction $f$ of people are either presently infected or have recovered and, therefore, are immune, with each new generation $R=(1-f) R_{0}$ people will become infected, rather than the larger $R_{0}$.

We, thus, see that the geometric progression or exponential growth described in the previous section is not a hard and fast rule, but simply a rough guide. In principle, more accurate mathematical models
Covid-19 in the United States

(continued)

After Northeastern states managed, through the efforts of their governors, to bring the numbers down, numbers shot up in other states, in particular in states in the South and Midwest, largely as a result of a premature reopening promoted by then President Trump. Unfortunately, in the US, mask wearing and social distancing have been turned into a political issue rather than a question of public health and plain common sense. The US subsequently experienced a serious second wave with hospital capacity overwhelmed in many parts of the country, with daily new cases peaking above 300000 on one day in January 2021.

Presently thanks to a massive vaccination campaign, with $49 \%$ of the population fully vaccinated and $56 \%$ having received at least one dose (22July 2021), the number of new cases has been brought down and life is starting to return to normal. However, because of the new more contagious delta variant, numbers are starting to rise again. 


\section{Mathematical Functions For Epidemiology}

Let $a>0$ be a positive real number and $x$ any real number. Then $a^{x}$ (read " $a$ to the power $x$," or more succinctly "a to the $x "$ ") is standard notation for the power function, which is included on most calculators and as part of most computer programming languages. The power function obeys the following relations:

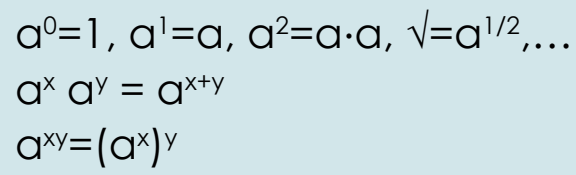

The exponential function, denoted as $\exp (\cdot)$, is a special case of the power function that pops up again and again

$\exp (x)=e^{x}$ where $e=2.71828 \ldots$ known as Euler's number

$\exp (x+y)=\exp (x) \exp (y)$

$\exp (0)=1, \exp (1)=e$

When $x$ is small (compared to one), $\exp (x) \approx 1+x$

The inverse of the exponential function is the natural logarithmic function, denoted as $\ln (\cdot)$, or sometimes as $\log _{e}(\cdot)$, which is read logarithm base $e$, so that

$\exp (\ln (\mathrm{x}))=\mathrm{x}$

$\ln (\exp (x))=x$

and the natural logarithm has the properties

$\ln (a b)=\ln (a)+\ln (b)$

$\ln \left(a^{x}\right)=x \ln (a)$

can be developed that could account for these complications, and this is precisely what applied mathematicians and epidemiologists do for a living. The success of such modelling is limited because human behaviour is hard to predict. Moreover, more sophisticated models include a large number of input parameters that are hard to determine from the data.

It is also possible that the virus will mutate, possibly in a way that will increase its spread. Mutation means that the virus goes through changes and perhaps becomes a stronger form of the virus. Any vaccine that is developed may not be useful for too long if the virus mutates, and upgraded vaccines may need to be developed. The virus can also change in how it affects those infected. It can become more or less virulent. This means that it becomes more or less severe. 


\section{What can we learn from the data?}

We already looked at some data showing that a geometric progression is not a bad model to start with in the short term. But let us look deeply at all of the data so far to see what we can learn about how the virus has spread and the impact that different policies have had on the numbers. In the previous section, we saw arguments around why the growth rate may be expected to change with time, at least somewhat. We now look at real data to see what has in fact happened.

The virus started in China and spread throughout the world. With globalisation and frequent air travel, the spread could not be avoided, although many people had hoped otherwise. Many countries closed their borders or tried screening travellers entering, for example by checking their body temperature, as had been done successfully with the SARS virus during the short-lived 2003 epidemic. These measures, however, were not effective enough for Covid-19, because not everyone with Covid-19 gets a fever, and moreover, infected people become infectious-meaning that they could spread the disease to others-before they start showing symptoms. Moreover, later on it was found that a subset of those infected never showed

People's perception of the seriousness of the disease and of their own probability of getting infected also plays a role in determining behaviour. any symptoms but, nevertheless, spread the disease to others. This explains, at least in part, why Covid-19 spread around the world and is now virtually everywhere, whereas SARS was successfully eradicated within a few months.

Different countries adopted different policies, which we will discuss further below. However, the general progression of the epidemic seemed to obey the following general trends:

1. In the beginning, although people had heard about the outbreak in China, they did not actually believe that it would reach their country, or they believed that their country would be spared. During the period (until mid-March 2020), we see a very rapid growth rate.

2. As numbers rose exponentially, and in many places, hospitals began to fill beyond capacity; people and governments reacted by imposing drastic measures such as lockdowns, which reduced-and in many cases also reversed-the growth rate. In some countries such as Australia, China, Israel, Vietnam, and New Zealand, to name a few, these measures succeeded in reversing and virtually eliminating the virus. In other places, a slowdown of the growth or modest reduction of the virus spread was achieved. 
3. In many places, however, because of the great economic cost of maintaining a lockdown and opposition from business interests, measures were loosened, accompanied by an increase in the growth rate.

The Covid-19 pandemic is an on-going story of which the ending cannot be predicted with any confidence. There are many plausible scenarios. Yet it is useful to look at the available data from different countries, looking in particular at how the growth rate has evolved over time and is correlated with the various policies implemented.

Probably the most useful way to plot the data is using a logarithmic scale for the daily number of cases. On a logarithmic scale, the instantaneous slope indicates the instantaneous growth rate, and multiplying by a constant factor is equivalent to sliding the curve upward (if $f>1$ ) or downward (if $f<1$ ). Exponential growth corresponds to a straight line of positive slope, and exponential decay corresponds to a negative slope.

It is important to keep in mind that the data available to us is not perfect. Ideally, we would like to know the exact number of new infections each day in a given country or province, but only a certain fraction of these new infections is reported

\section{Daily new confirmed COVID-19 cases}

Shown is the rolling 7-day average. The number of confirmed cases is lower than the number of actual cases, the main reason for that is limited testing.

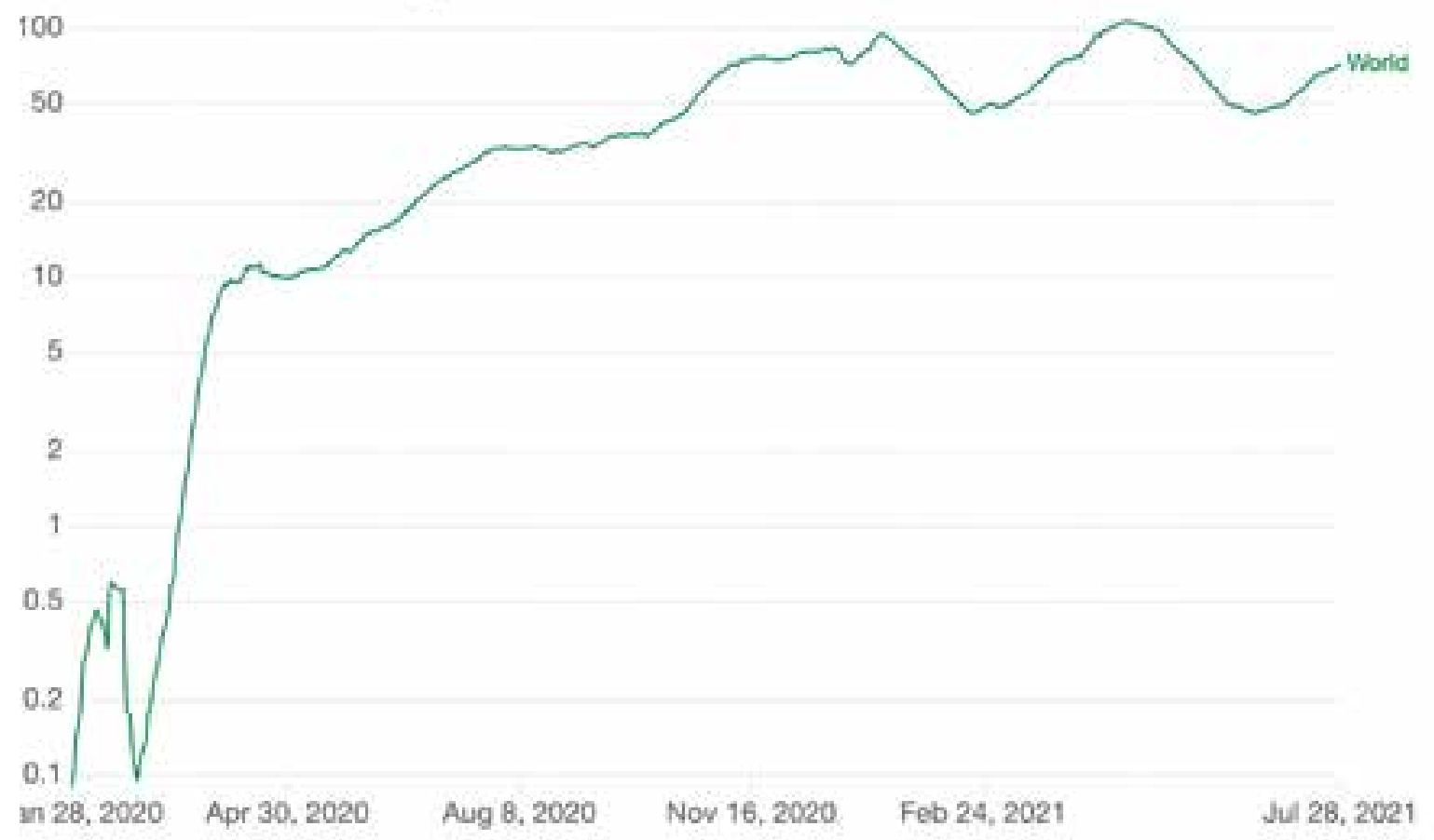

Figure 5.3: Daily new cases worldwide (since July 2021)

Source: https://ourworldindata.org/covid-cases

Creative commons license 
and, consequently, makes it into the official statistics. But it would be reasonable to assume that the under-reporting takes place almost everywhere, so some predictions can still be made from reported cases. Covid-19, at least shortly after someone has initially been infected, does not have very specific symptoms. Consequently, the only reliable diagnostic test is detection of the viral RNA in the nasal mucus through an RT-PCR test, which, at least initially, was not widely available. Consequently, we have every reason to believe that the actual number of infections is being undercounted by some factor not so easy to determine. Because of limited testing capacity, many people who believe that they may have the disease are not able to be tested. In any case, because of costs, some governments only test people who show symptoms. Moreover, as we have recently learned, some people who become infected do not have any symptoms, yet are able to infect others and, thus, spread the disease. Despite these challenges, the imperfect data available to us is better than no data at all. Otherwise, we would have a situation where everyone is left to speculate according to their whims and personal preferences.

We shall first look at the data for a few representative countries for the number of new cases against time, later looking at some other types of complementary data. There are many ways to plot the data and each method highlights a different

\section{Daily new confirmed COVID-19 cases}

Shown is the rolling 7-day average. The number of confirmed cases is lower than the number of actual cases, the main reason for that is limited testing.

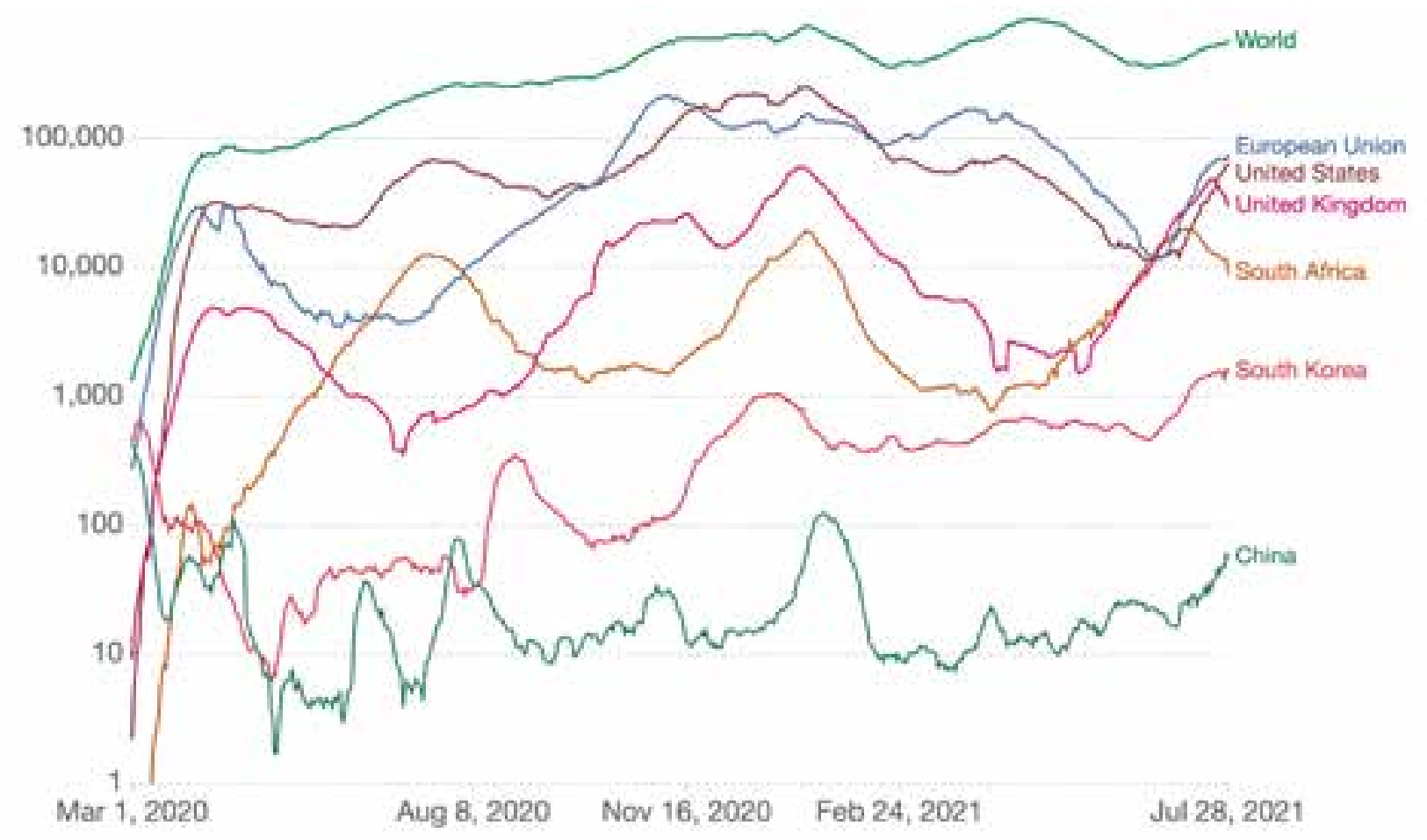

Figure 5.4: Daily new cases-A few representative countries and regions

Source: https://ourworldindata.org/covid-cases

Creative commons license. 
aspect of the data. Nevertheless, one common tool is using a logarithmic scale for the number of infections. Logarithms (regardless of their base) have the property $\log (a b)=\log (a)+\log (b)$. This means that multiplying a curve by a constant factor slides it either up or down, depending on whether the factor is greater than or less than one, but the shape of the curve is not changed. A logarithmic vertical scale allows us to focus on the evolution of the growth (or decay) rate of the infection. Exponential growth on a log scale is represented by a straight line of positive slope, the magnitude of the slope being equal to the growth rate. Similarly, exponential decay is represented by a straight line of negative slope, the magnitude of the slope representing the decay rate. In general, the curve will not be straight, indicating a changing growth (or decay) rate.

With some understanding of the mathematics used in modelling, let us look at some data, like the daily infection rate for the entire world, in order to identify a few general trends. We begin at 23 Jan 2020, when there were 100 cases per day (in order to avoid noise and other sampling artifacts). Noise refers to changes in the data caused

\section{Daily new confirmed COVID-19 cases}

Shown is the rolling 7-day average. The number of confirmed cases is lower than the number of actual cases, the main reason for that is limited testing.

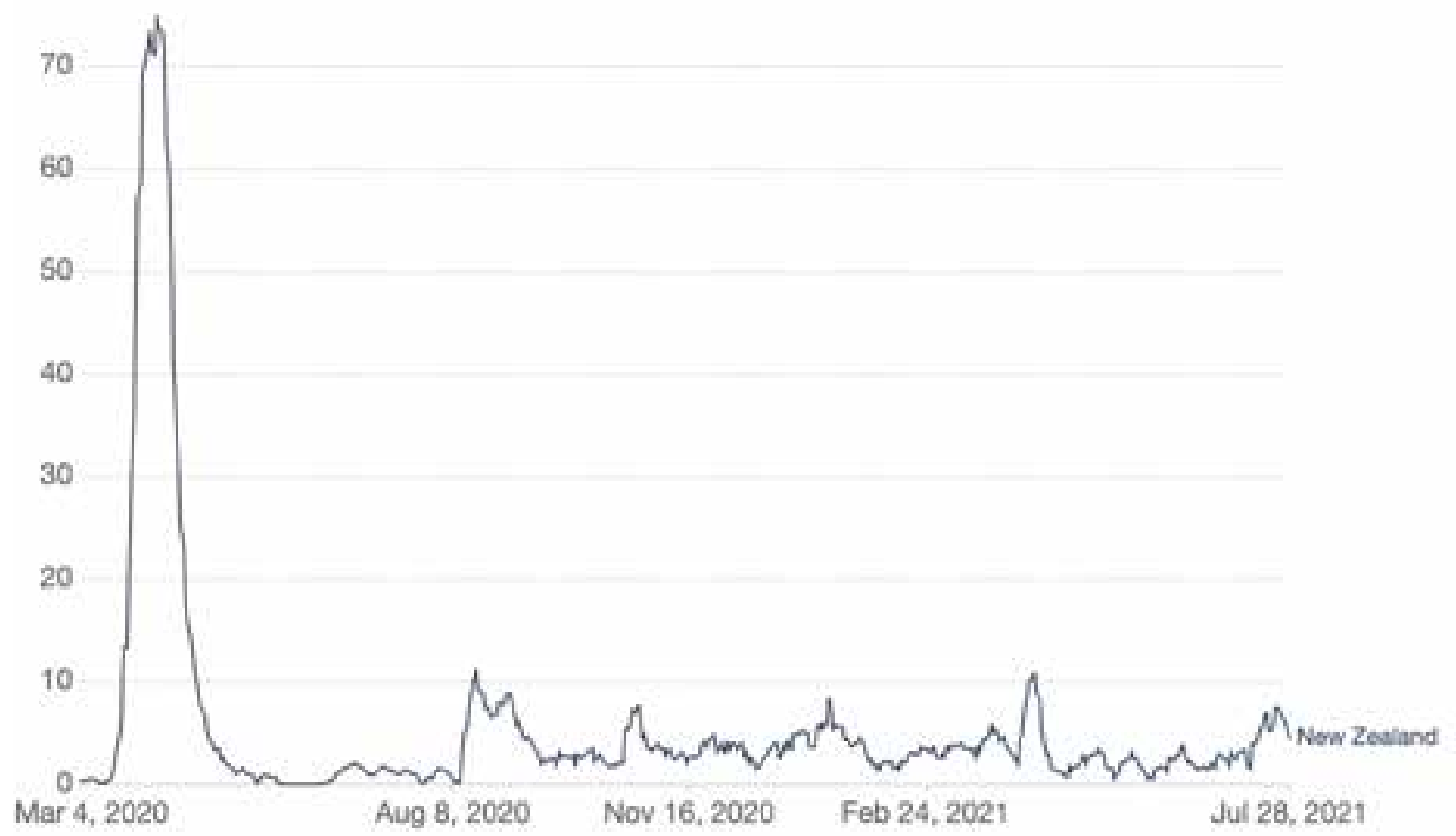

Figure 5.5: New Zealand daily new confirmed COVID-19 cases

Source: https://ourworldindata.org/covid-cases

Creative commons license. 
by other influences not related to the disease. From $24 \mathrm{Feb} 2020$ through to 24 March 2020, we observe an 83-fold increase, corresponding to a doubling of the infection rate every 4.4 days. During this time, China was able to bring down the number of infections through drastic measures. However, the pandemic had already spread to Europe, which was slow to react, leading to a massive increase in infections, until Europe put in place a lockdown, leading to a massive drop in the number of daily infections. The US, however, was slow to react and now leads the world in new infections and deaths.

The data exhibits weekly ripples, primarily explained by delays in reporting cases over the weekend. In some countries there were delays in getting test results as these were not released in a regular manner. By contrast, in the 76 days from 11 May 2020 through to 26 July 2020, the worldwide daily infection rate increased by a factor of 3.33, corresponding to a doubling every 44 days. Since then, the worldwide daily number of new cases has continued to climb. [There are many compilations of worldwide Covid-19 data (see references at end). The plots here are taken from the website: https://ourworldindata.org/covid-cases ]

China, where the initial outbreak occurred, seems to have brought down and controlled its numbers. The European Union was the next region hit by the pandemic and, for a while, led the world numbers, before it was overtaken by the United States. South Africa (as well as the rest of Africa) was a latecomer and, after peaking in mid-June, saw a substantial decline followed by a new peak, or 'second wave'.

\section{New Zealand: An Apparent Success Story.}

Some would argue that China's success in reducing and reversing the spread of the virus may be due to its unique social system and, thus, cannot be repeated elsewhere. Very strict government control steps were followed by a compliant population. However, New Zealand, which has a social system quite unlike China, has, at least to date, also been able to almost extinguish the virus. It is worth noting that the population of China was about 1400 million in 2020, while the population of New Zealand is merely about 5 million, so the scales are vastly different. A small population is obviously easier to manage than a huge one. Other examples where the numbers have been brought down close to zero include Australia and Vietnam, although Australia did suffer a second wave. Israel was successful in bringing down the numbers at the end of May 2020, but has since been less successful.

We could show more plots and different ways of plotting the data, and there is a lot more to be learned from the data. But, instead, we invite readers to explore the data by themselves using the internet and to draw their own conclusions. In any case, by the time this booklet goes to print, the above data will be out of date, so the various cited websites are the best source of up-to-date information. 
Good websites are: https://www.worldometers.info/coronavirus/, https:// ourworldindata.org/covid-cases for worldwide data, and https://sacoronavirus. co.za/, https://www.covid19sa.org/ for data from South Africa, nationally as well as by province.

Above, we focused on daily case numbers based on test results, but it is good practice to look at as many different types of data as possible to look for corroboration (where there is agreement) as well as possible inconsistencies (where there are differences). This is necessary when we know the data is not perfect but we want to put together the most reliable picture of what is going on.

Other types of data are number of hospitalisations due to Covid-19, deaths as a result of Covid-19, number of people admitted to intensive care for Covid-19, fraction of positive test results, and excess mortality (number of deaths). Each tells us something slightly different and has its own pros and cons concerning accuracy. While there might be great variations on whether people with less serious Covid-19 are tested, those requiring hospitalisation are more likely to be counted in the same way, and counting the more serious cases, requiring more scarce medical resources is of great interest in its own right. The fraction of positive test results is, to some extent, less dependent on how much testing takes place. Assuming that a varying fraction of people with Covid-19-like symptoms gets tested, the fraction of positives is more likely to reflect the true increase in the prevalence of new infections than the raw number of positive test results. Mortality data (death rate), which may be expected to lag the number of new infections by several weeks, is perhaps more reliable for measuring the shape of the curve, assuming that the cause of death is usually determined correctly. These data, however, are not completely unambiguous, meaning they can be interpreted in different ways. Many, but not all, people dying from Covid-19 have co-morbidities (meaning other diseases at the same time). Consequently, there may be several causes of death, and no right answer in how to identify the most important cause of death. Whatever the actual cause of death, hospitals would have the ability to tell if the patient was Covid positive or not.

Looking for excess mortality (higher than normal death rates) sidesteps this problem and also gives a way to ensure our deductions are sensible. In situations where hospitals are overwhelmed, as was the case in Italy in late March 2020, comparing the rate of deaths with previous years allows for unrecorded Covid-19 deaths to be detected, as can be seen by comparing the 2020 curve to previous years. 


\section{Excess mortality during COVID-19: Deaths from all causes compared to previous years, all ages}

Shown is how the number of weekly deaths in 2020 - 2021 differs as a percentage from the average number of deaths in the same week over the years 2015-2019. The metric is called the P-score. We do not show data from the most recent weeks because it is incomplete due to delays in death reporting.

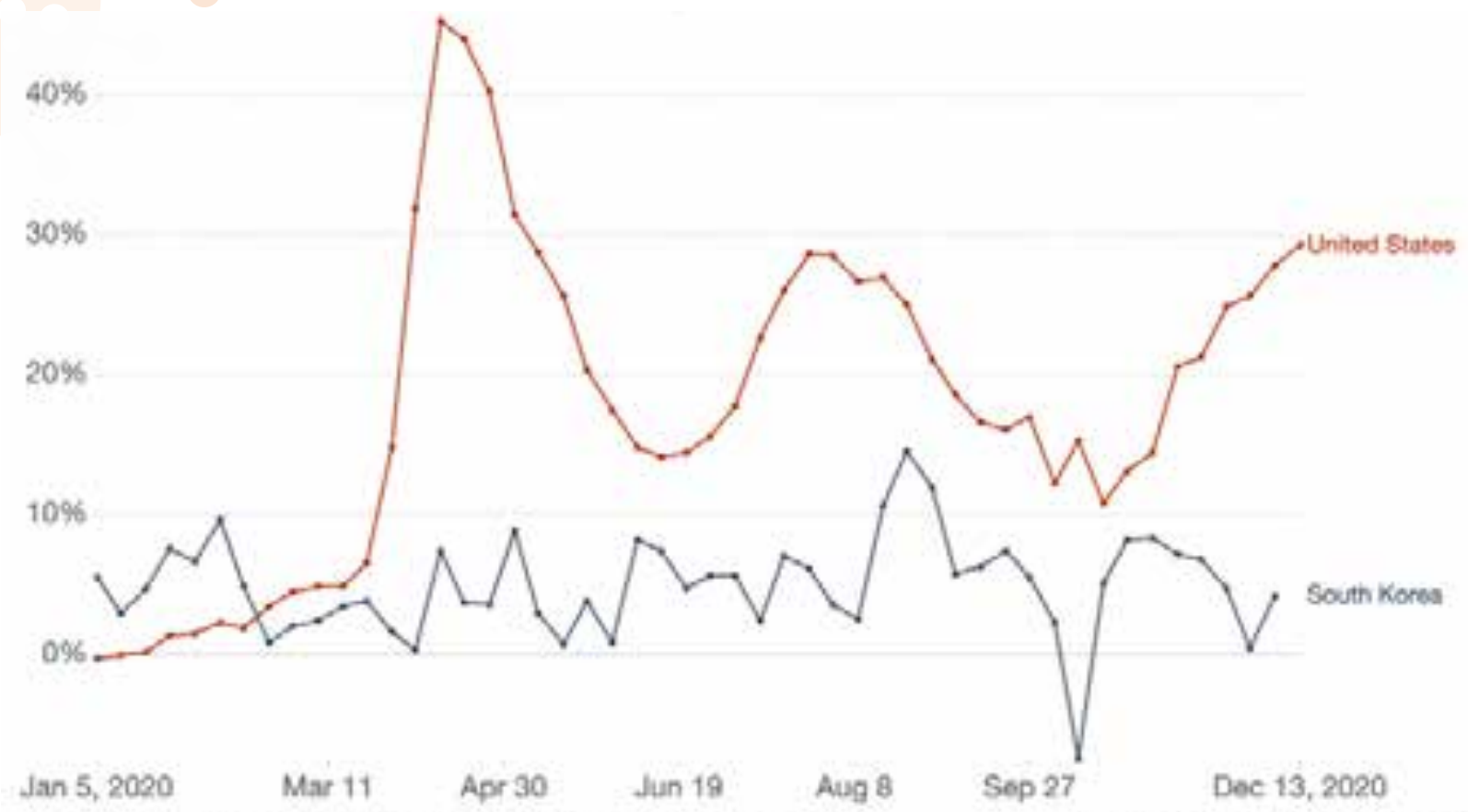

Figure 5.6: Comparison of excess deaths in the United States and South Korea

Source: https://ourworldindata.org/covid-cases

Creative commons license. Human mortality database (2021), UK Office forNational Statistics (2020)

Weekly mortality data can be compared with previous years to estimate the number of deaths due to Covid-19 by another method. We observe three peaks of excess mortality in the United States, the highest reaching 45\%, whereas in South Korea, even though 2020 had some excess mortality, the rate averages to around 5\%. Examining excess death data, rather than the statistics of deaths formally attributed to Covid-19, includes people who died of Covid-19 without being diagnosed as such. However, part of this excess mortality may be due to people who died of other causes, perhaps because they did not access healthcare, either because of fear of getting infected or because of medical resources being diverted to dealing with Covid-19 patients. 


\section{South Africa weekly Deaths from all causes 1+ years: 29 Dec 2019 - 16 Jan 2021}

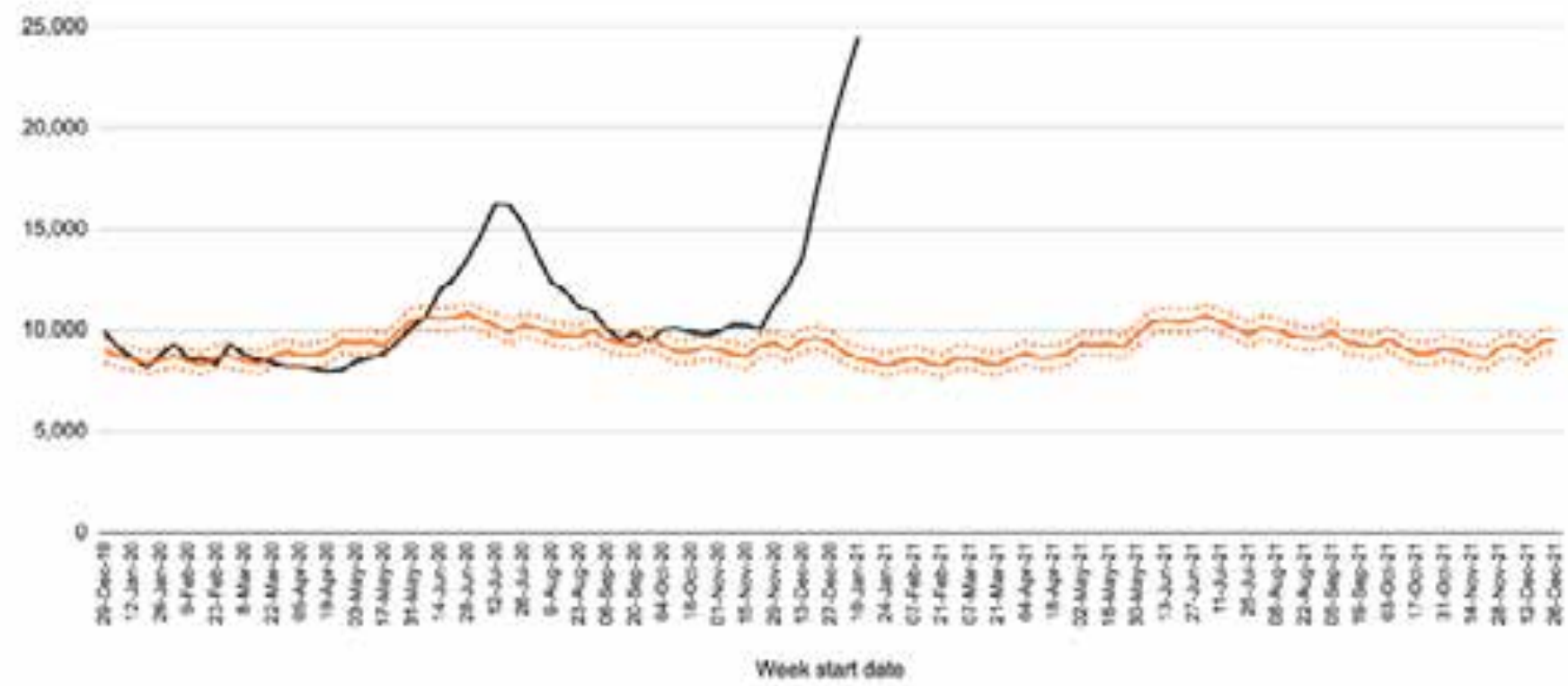

Figure 5.7: South African excess mortality data

Source: South African Medical Research Council https://www.samrc.ac.za/reports/report-weeklydeaths-south-africa

The solid red line indicates the weekly mortality prediction based on previous years, with the range expected from statistical fluctuations indicated by the dotted red lines. The solid black line indicates the 2020 data. $R_{0}$ is defined to be the average number of people newly infected by each infected person. As explained above, when a sizable fraction of the population is presently infected or has become immune, the number of new infections is smaller and denoted by $R$.

In the simplest model:

$$
R=(1-f) R_{0}
$$

where $f$ is the fraction of people already infected or immune.

Because $R_{0}$ depends on people's behaviour, it can be reduced by the various measures outlined in the previous chapter: masks, quarantines, avoiding congregating in large groups, social distancing, testing and isolating.

\section{What is 'herd immunity'?}

Above, we explained how the virus will eventually go away if $R$ drops below one. Happily, making the virus go away does not require every transmission of the virus from one person to another to be stopped. It is enough to lower the rate of infection so that each infected person infects, on average, fewer than one other person. Mathematically, this means that the reproduction number drops below one. In this case, with time, fewer and fewer people are infected, and eventually the number 
of people infected will drop to zero. If $R$ is only slightly less than one, this will take a very long time. Many generations of infection will be needed to make the virus go away. But if $R$ is much less than one, a small number of generations will be needed.

Globalisation complicates the eradication of the virus by such means. A country may get their numbers down to zero or close to zero by a strict lockdown over several months, as occurred in China. If people from other countries where the infection persists were not travelling into the country and creating new infections, the lockdown could be lifted and people could go back to their old ways without the virus. $R_{0}$ can be much greater than one as long as the number of people infected is zero. But introducing one new infection can restart the exponential growth described above. Consequently, there is great interest for all countries to work together on a worldwide strategy for eliminating the virus everywhere.

As we mentioned above, it is most likely that once someone has been infected with Covid-19 and recovers, they are immune from being re-infected by the virus for a very long time, possibly for life. This is the case for many (but not all) viruses, so this is our present best guess. Moreover, immunity can be conferred by a vaccine, as described above, rather than by actual infection. The more people are immune, the lower $\mathrm{R}$ becomes compared to $\mathrm{R}_{0}$ so $\mathrm{R}$ can be made smaller than one once a certain critical fraction of the population has acquired an immunity, either from actual infection or from having taken a vaccine. If everyone is the same, the fraction of people who need to be immune is not $100 \%$. It is sufficient for (1-f) $\mathrm{R}_{0}$ to be smaller than one. When at least this fraction of immunity has been achieved, the rest of the population acquires what is sometimes called 'herd immunity'.

In the case of vaccines, the fraction vaccinated never approaches exactly one (or $100 \%$ ), and all people do not respond to the vaccine in the same way. For many vaccines, some people acquire an immunity while others do not, especially older people whose immune

\section{Covid-19 on the Diamond Princess Cruise Ship}

The fateful voyage of the Diamond Princess, a British cruise ship that carried approximately 3700 passengers and crew, starting in Yokohama on 20 January 2020, demonstrated how easily the disease could spread in a closed environment. This incident also provides an estimate of the fraction of asymptomatic cases because everyone aboard was eventually tested for Covid-19. When the ship departed, one passenger who had been to China had developed a cough but boarded anyway. After disembarking in Hong Kong, he tested positive for Covid-19. Despite being notified immediately by the Hong Kong public health authorities, the cruise ship line delayed taking action and notifying the passengers. Partying in close quarters on the ship continued. Upon learning of the outbreak, the Japanese government decided to quarantine the passengers and crew on the ship for 14 days. Initially 30 ill passengers and crew were tested, giving 10 positives.

(continued on page 57) 
systems are typically less responsive. But thanks to 'herd immunity', a vaccine can protect an entire population without being $100 \%$ effective. It is enough for a certain critical fraction of the population to be vaccinated, and then everyone is protected. It is also important that there not be pockets of sub-populations with a sub-critical fraction of people vaccinated, as has sometimes occurred due to rumours of the dangers of vaccination causing parents to fail to vaccinate their children. There have recently been a number of outbreaks of measles because of this. Someone travels to a country were measles is still prevalent and returns, infecting a sub-community, which then suffers an outbreak.

\section{How is Covid-19 likely to end?}

This is the big question and nobody knows the answer for sure. However, a number of scenarios can be sketched of how this on-going story may play out. With time we will find out which scenario unfolds.

\section{Scenario I-A good vaccine comes along soon}

Let us start with the happy ending-or best case scenario. Presently many different vaccines are in the process of being developed and tested, some of which are now available.

Nevertheless, a number of challenges remain. Even in the wealthiest countries, owing to various challenges, it will be many months until enough people have been vaccinated to substantially stem the continuation of the pandemic.

The vaccine must be produced in massive quantities, with billions of doses, and distributed throughout the world. Having just a few countries vaccinate its population will not suffice to eradicate the disease. The immunity may also wear off, meaning that with time infections will return to the countries initially benefiting from the vaccination.

\section{Covid-19 on the Diamond Princess Cruise Ship}

(continued)

In the ensuing days, the isolation measures on the ship proved inadequate and, as more and more people were tested, the number of positives climbed. In the end everyone was tested, which showed a $19.2 \%$ positive rate. Among the positives, $42 \%$ were asymptomatic. Over 700 people were infected and 14 people ultimately died. 
Another danger in this case has to do with the possibility of the virus mutating. All viruses mutate to some extent, and some mutations are harmless. But the virus may mutate into a new form against which the vaccine no longer works.

For decades, scientists have sought to develop a vaccine against HIV-1, and there have been some partial successes. But each time the virus has mutated into a new form against which the vaccine proved to be powerless. This is because HIV-1 mutates particularly quickly. This property has made it challenging to develop effective

\section{Measles cases in the United States 1944 - 2007}

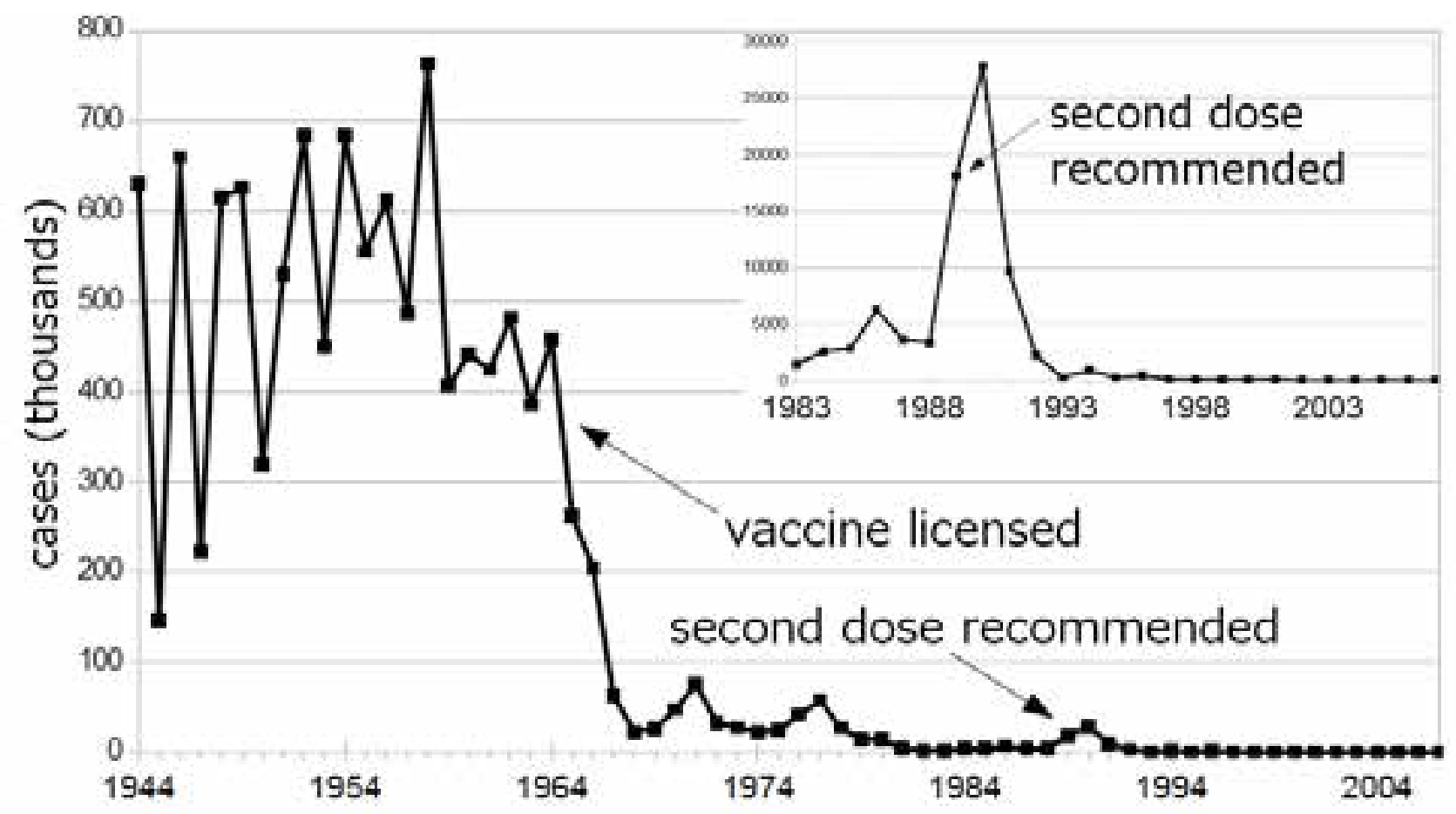

Figure 5.8: Incidence of measles in the United States

Source: https://commons.wikimedia.org/wiki/File:Measles_US_1944-2007_inset.png

drugs against HIV. The early drugs proved effective at first. But after some time a resistant strain would develop within the patient against which the monotherapy proved powerless. It is worth noting that South African scientists have been at the forefront of research in HIV research largely because of the local population having a high rate of infection. Happily, coronaviruses mutate more slowly. Whereas the reverse transcription of $\mathrm{HIV}$ is extremely error prone, producing many mutations, the RNA replication process of the coronavirus includes an error correction mechanism leading to a comparatively low mutation rate.

Thus, we see that there is hope for this optimistic outcome to be realised eventually but it is by no means guaranteed. 


\section{Scenario II-Covid-19 becomes endemic}

If the population remained fixed and infected people became immune for life, the disease would eventually die out as a result of herd immunity, as described above, although the final stage of extinction would occur very slowly. It is possible that seasonal effects, of little relevance when $R$ is much greater than one, could speed up a possible extinction of the virus. But a population over a long timescale is not a fixed population. Older people die and are replaced by new births, even if the total population number does not grow. Consequently, the new births will drive the susceptible fraction above the critical value, and new outbreaks will occur.

This is not just theoretical speculation but describes the situation for measles before a vaccine came along in the mid-1960s. Abundant, high quality data is available for measles, as shown in Figure 5.8.

The number of cases saw a seasonal periodicity until a vaccine was discovered in the mid-1960s followed by a program of mass vaccination, which virtually eliminated the disease. However, recently, due to false information claiming that the vaccine is not safe and leading to pockets of unvaccinated children, new outbreaks have resulted.

The somewhat irregular spikes showing a periodicity of one or two years are most likely due to seasonal effects, which cause R to oscillate with a yearly pattern. We have not yet observed any seasonal variation in Covid-19 despite speculation that it would "go away like the flu", which away from the tropics is a wintertime phenomenon. In the summer of each hemisphere there are hardly any cases of the flu, but in winter the new strains propagate away from the tropical regions with a remarkable regularity. In the case of Covid-19, if $\mathrm{R}$ hovers around one, seasonal effects could become important.

This scenario would not be a happy ending. But neither would it be the worst-case outcome. People would

\section{Covid-19 in France}

After China, Europe (particularly France, Italy, Spain, and later the United Kingdom) was hit hard by the Covid-19 pandemic. The number of new cases saw a peak around the beginning of April 2020 but, by means of lockdowns and other measures, European countries were successful in greatly reducing the number of daily new cases toward Summer 2020. However, starting in Autumn, the number of cases has steadily risen, resulting in a new series of lockdowns.

After the number of new cases was doubling every few days, the French government announced a lockdown on 11 March 2020 that lasted till mid-May. All people except essential workers were confined to their homes. All stores, except grocery stores and pharmacies, were closed. These measures succeeded in bringing the daily new case numbers down to about a tenth of their peak value, at which point a gradual reopening was put in place.

(continued on page 60) 
be infected at an earlier age, when Covid-19 is least serious. But to reach this steady-state situation, a lot of people would have to become infected, and many people would die. And as we are learning, those who recover from the more serious cases of Covid-19 often suffer serious health problems that do not go away.

This scenario assumes that the virus retains its present character over the years to come.

\section{Scenario III-The virus mutates into a new strain that is much more infectious and deadly}

Covid-19 is the worst disease outbreak in the last 100 years. Competition includes the so-called Spanish Influenza of 1918. Although many of the infectious diseases of the past, against which effective vaccines are now commonplace, have been around for at least a few hundred years, viruses evolve and new strains come along. For influenza, for example, each year one or more new strains appear due to mutations and different combinations of viral genes. This is why each year a new vaccine against influenza must be formulated, tailored to the strains thought to become prevalent during that year. Vaccinating against influenza, because of its rapidly mutating character, is akin to striking a moving target. Other viruses, prevalent among other species such as birds, pigs and bats, through mutation, change species and become infectious for humans. This is what happened for Covid-19, which has been shown to be genetically extremely similar to coronaviruses prevalent among bats, and which possibly jumped to humans via some other intermediate species.

We thus see that viruses evolve, and they evolve quickly compared to other kinds of living organisms. SARS- and MERS-COVs are other coronaviruses similar to SARSCoV-2, but much more deadly. The case fatality rates for MERS, SARS, and SARS-COV-2 are given as 37\%, $9.2 \%$, and $3.8 \%$, respectively, although the last figure is the least certain due to difficulties in identifying all Covid-19 cases. Although all SARS and MERS always

\section{Covid-19 in France}

(continued)

One of the drivers for the lockdown was the fact that hospitals were being overrun, in terms of number of beds, trained medical personnel, and capacity in intensive care wards. Patients in the East of France had to be sent to hospitals elsewhere in the country for lack of local hospital capacity. In the Paris metropolitan area, hospitals were very close to exceeding capacity. At first, there was also a shortage of masks and other personal protective equipment for medical personnel.

France, however, has experienced a second wave, as has most of Europe and the United States, leading to a new lockdown, followed by curfews and other restrictions.

France's second wave peaked around the beginning of November 2020, followed by a third wave that peaked around mid-April 2021. As of writing we seem to be seeing the start of a fourth wave driven by the delta variant. Currently $42 \%$ of the French population is fully vaccinated, and the current government strategy focuses on increasing the fraction of the population vaccinated. 
lead to severe symptoms, as discussed above, this is not the case for SARS-CoV-2, which has proved much more contagious. A mutation or recombination event by which Covid-19 becomes more deadly cannot be ruled out. Something of this sort could be described as the worst case scenario.

\section{New strains}

Recently new strains of the virus that spread more rapidly than the old strains have appeared in the United Kingdom, Brazil, and South Africa. The replication of viruses implies the copying of its genetic information encoded in the viral RNA. During this process, errors may occur by chance. Amino acids may be substituted, deleted, or inserted by mistake. Such deviations from the original in the copied RNA are known as mutations. Some mutations may be advantageous to the virus's survival, by improving the 'fitness' of the virus. Others will hardly affect the virus's survival, and yet others will be harmful to its survival and consequently will disappear. Of course, scientists are worried about the mutations that make the virus 'stronger' and more dangerous to the host-that is, us.

Since the beginning of the pandemic, scientists have already observed a few thousand mutations of the virus. Three mutations, however, have alarmed the medical community, as they have increased the virus's ability to infect the hosts. These are the mutations that help the virus spread more easily and have induced a surge in Covid-19 cases worldwide.

One of these variants, originally called 501Y. V2, then B.1.351 and now Beta, was discovered in South Africa and described by Prof Tulio de Oliveira's team at KRISP in Durban. Doctors had noticed a surge in Covid-19 cases in the Eastern Cape and the genomic investigation of its cause revealed the new variant of the virus. Beta has now

The higher transmissibility of the new variant is undisputable. Early evidence suggests that the new variant does not cause more severe forms of the disease. become the dominant version of the virus in South Africa. The higher transmissibility of the new variant is indisputable. Early evidence suggests that the new variant does not cause more severe forms of the disease. Scientists are also investigating if the available vaccines will offer protection from the new variant and if antibodies from previous infection with the original variant will suppress the new variant.

In the meantime, two more variants have been observed that share similar characteristics to Beta. The variant detected first in the UK is called Alpha (formerly 501Y.V1 or B.1.1.7) and has been detected in many other countries since then. Another variant, P.1 (501Y.V3), was observed first in Brazil. It is imperative to monitor the evolution of these variants globally and understand their characteristics. 
The Delta variant (B.1.617.2), first discovered in India in late 2020 because of its enhanced ability to spread, has become dominant in many countries already and may become the dominant variant everywhere.

Modelling the spread of infectious diseases is difficult. Professional modellers often disagree with each other due to the use of different simplifying assumptions and interpretations of the data. Moreover, individual models often predict a range of outcomes rather than making a single precise prediction. The range of predictions is often more reliable than the predictions of any single model.

Above we described exponential growth, which provides good short-term predictions, but applied mathematicians have developed more sophisticated models called SIR models, in which individuals are pooled into susceptible, infected, and recovered classes. Mathematical models predict how individuals pass from one class to another. Although these models are helpful, many of the details that enter into the models rely on imperfect data, making it difficult to establish reliable long-term predictions. Nevertheless, such modelling helps us better understand the range of possibilities, and the impact of policy on outcomes.

Not being able to predict the future accurately is not unique to epidemiology. In economics and business,

Not being able to predict the future accurately is not unique to epidemiology. predicting inflation, exchange rates, prices and demand for particular goods, etc. is indispensable to making sound business decisions. Yet, precise predictions cannot be made, and the projections made are often way off.

\section{If one catches Covid-19, what is the probability of death? Which factors affect mortality?}

These are important questions but not so easy to answer precisely. This is because the data available to us is not exactly the data that we would like to have. We would like to count all the cases of persons who are infected. However, the reporting of more serious cases is more complete, and cases of those who are infected but show no symptoms are almost never reported. This causes the total number of people infected to be under-reported. On the other hand, deaths lag new cases by several weeks. When the daily number of cases is growing, this causes the mortality (death rate) fraction to be under counted. Various attempts can be made to model the mortality (death rate) for those infected. Moreover, beyond these problems arising from the data, the mortality fraction per infection depends on a number of factors: age, sex, co-morbidities, quality of medical care, to name just a few examples. Mortality rates from different countries will differ, in part because of different mixes of these factors. It also appears that the chance of dying has been going down, likely because doctors are learning how to better treat Covid-19 patients. 
Some drugs used for other diseases have been useful in reducing deaths in Covid-19 patients. These include Remdesevir and dexamethasone, where good outcomes for patients were found.
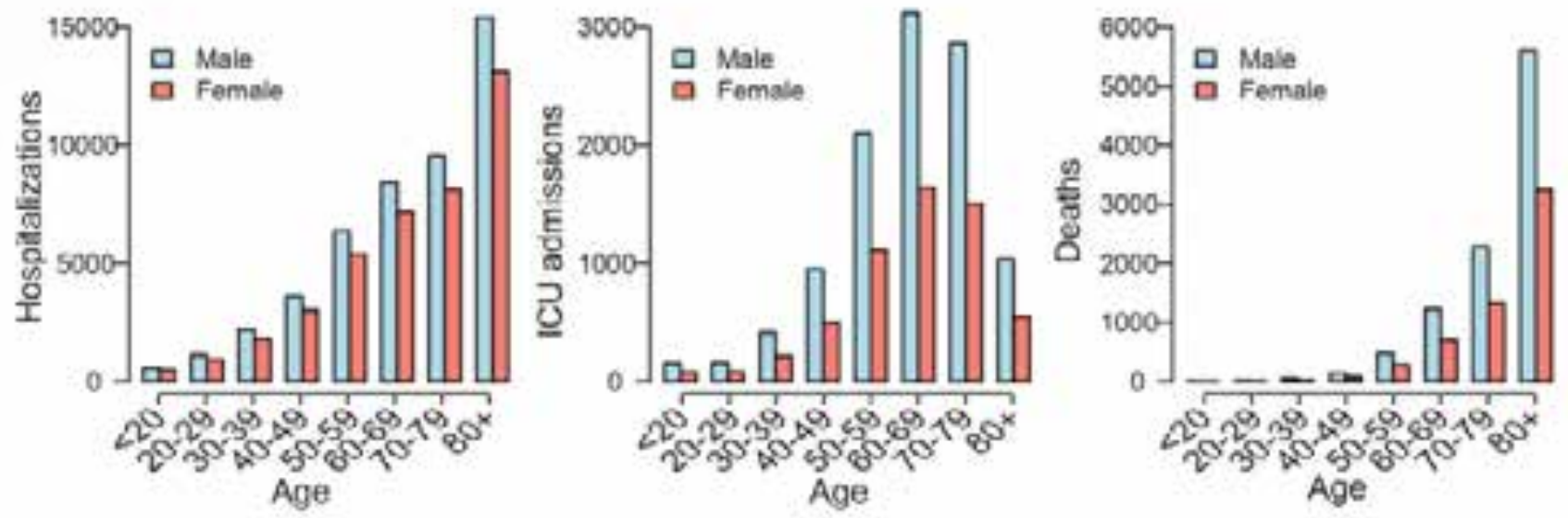

Figure 5.9: French Covid-19 data and age

The left, middle, and right panels show the distribution of hospitalisations, intensive care unit (ICU) admissions, and deaths in France, respectively, classified according to age and gender.

Reprinted with permission: H. Salje et al., "Estimating the Burden of SARS-CoV-2 in France," Science 369 (2020) 208 (10July 2020)

Age and co-morbidities are important factors in determining how sick someone who gets infected will become and whether they are likely to die. As a rule, older people are more likely to become very sick, and also to die. Moreover, people who suffer from other health problems (also known as 'co-morbidities') are more likely to become severely sick and to die. Such health problems include: diabetes, obesity, respiratory disease, reduced immune system function, to name a few examples. These conditions can increase the chance of a bad outcome by a factor of up to 3 or more. A study published in the prestigious scientific journal Nature using data collected in the United Kingdom, explored how different factors increased or decreased the chance of dying from Covid-19. This study found that men infected are about 1.6 times as likely to die as women infected, and found the following increase in the chance of dying as a function of age, using the 50-59 age group as a reference.

\begin{tabular}{|c|c|}
\hline Age group & Increase in probability of death \\
\hline $18-39$ & 0.05 \\
\hline $40-49$ & 0.28 \\
\hline $50-59$ & 1.0 (reference) \\
\hline $60-69$ & 2.79 \\
\hline $70-79$ & 8.62 \\
\hline $80+$ & 38.29 \\
\hline
\end{tabular}


In summary, young people and people without other medical conditions have less to worry about regarding their outcome if they get infected. This however does not mean that they should not be careful, because they can infect the older people around them and, in rare situations, very young people have mysteriously died.

South African Population (2017)

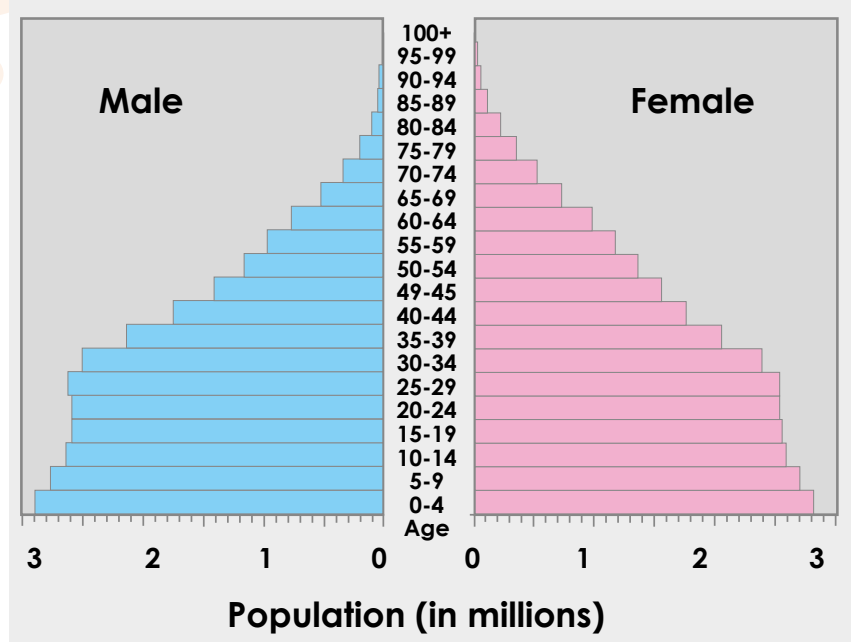

France Population (2017)

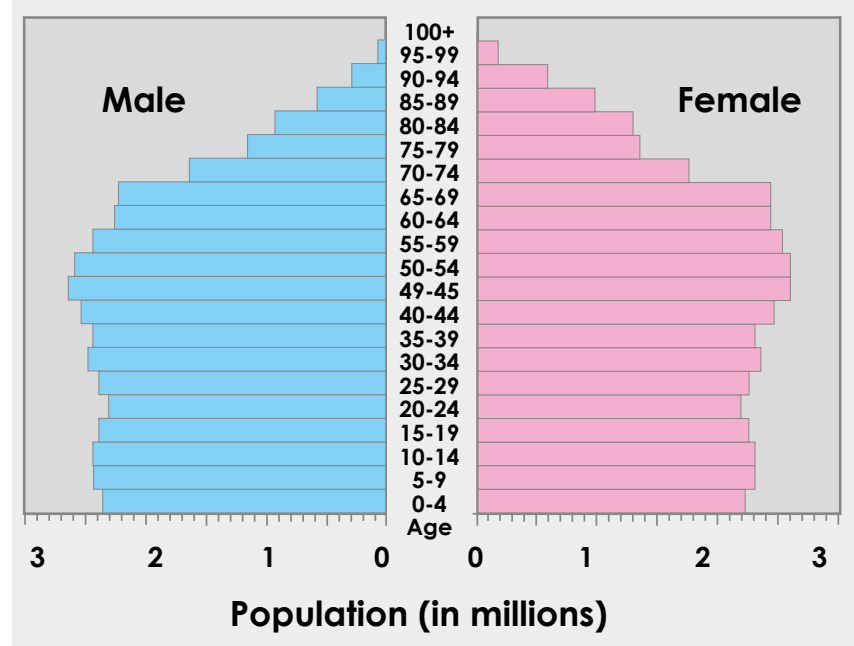

Figure 5.10: Age pyramid: South Africa vs France

Source: https://commons.wikimedia.org/wiki/File:South_Africapop.svg: https://commons.wikimedia.org/wiki/File:Francepop. svg 


\section{CHAPTER 6}

\section{Viruses in the Human Body}

To understand what a virus is we need to see it in a broader context. Our Universe and our Earth are approximately 13.7 and 4.5 billion years old, respectively. Both living and non-living matter are made of atoms. There are many theories of how life began on Earth from non-life. The first living organisms were archaea and bacteria, which we call prokaryotes. All complex life on Earth shares a common ancestor, a eukaryotic cell that arose from bacteria on just one occasion approximately 2 billion years ago. The questions are what are viruses? Where do viruses fit into this scheme? Why do viruses spread so fast and cause so much harm? How does the body's immune system respond to a viral infection and what is the response of the medical establishment to a global pandemic such as the coronavirus pandemic we are currently experiencing? These are questions that this chapter attempts to answer.

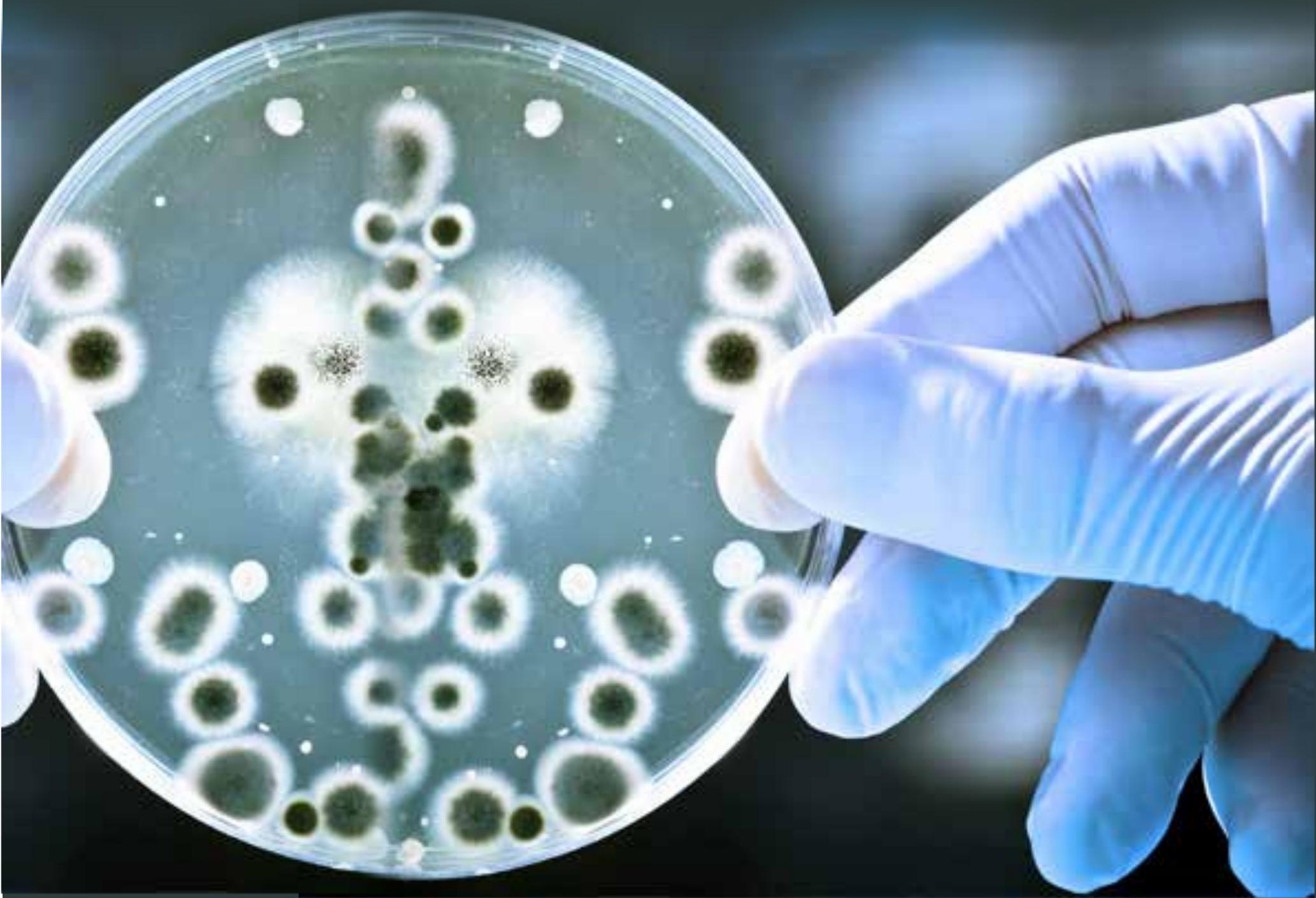




\section{What is Covid-19?}

Covid-19 is short for coronavirus disease 2019, a viral infection that has afflicted people all over the world. When a disease spreads worldwide, it becomes known as a pandemic. An epidemic, on the other hand, is an infectious disease that has spread throughout a community at a particular time without spreading worldwide. We commonly speak, for example, of a 'flu epidemic', 'the HIV-AIDS' epidemic in sub-Saharan Africa, an 'epidemic of drug abuse' in a particular area, or we say, 'there is an epidemic of diabetes in the Indian population of KwaZulu-Natal'. The main actor in our entire story is the new SARS-CoV-2 coronavirus, the cause of the Covid-19 pandemic.

Because we are dealing with a new virus which is causing worldwide devastation, scientists throughout the world have been studying the virus in an attempt to produce a vaccine. Before trying to understand the origin and manner in which the virus infects humans to produce the clinical symptoms of the Covid disease, it is necessary to first put things into context.

Everything you look at, whether it is a rock, a virus, the air we breathe, a tree or human being, is made of atoms. Different arrangements of atoms produce different forms of matter, some alive (trees and humans) and some not (air, rocks, etc.), and some that sit somewhere in between, seemingly not living, but not non-living either. These include the viruses.

Atoms join in various combinations to form molecules, for example, two hydrogen atoms and one oxygen atom combine by covalent bonding to form water. Water is an example of a simple molecule. Atoms can also form more complex molecules (and macromolecules, which are very large and generally put together in living organisms). Imagine a delicious piece of steak. What are the components of that piece of meat? You will find that it is made up of both macromolecules and smaller molecules. The macromolecules are proteins and nucleic acids (DNA and RNA and, in this case, coronavirus is an RNA virus), or assemblies of complex lipids and carbohydrates. These are organic compounds made up largely of

Everything you look at, whether it is a rock, a virus, the air we breathe, a tree or human being, is made of atoms. carbon $(C)$, hydrogen $(H)$, nitrogen $(N)$, oxygen

(O) and phosphorous (P) atoms. The steak will also have smaller molecules such as large amounts of water $\left(\mathrm{H}_{2} \mathrm{O}\right)$ and any number of small organic compounds and inorganic ions such as sodium $(\mathrm{Na}+)$, potassium $(\mathrm{K}+)$, chloride $(\mathrm{Cl}-)$ and other ions.

The organic and inorganic molecules are arranged to form structures called cells. On our planet, this first happened approximately four billion years ago. The earliest cellular structures were the prokaryotes (cells with no nucleus, with the genetic 
material floating around in the cytoplasm). We know prokaryotes very well today because, like viruses, some of them cause serious diseases in our bodies. Others, however, are beneficial to humans (for example, those living in our large intestines or on our skin). However, prokaryotes differ from viruses because they have the property of life: they are metabolically active, which facilitates their independent reproduction. Viruses are often considered non-living as they can reproduce only inside a host cell. They require the machinery of the host cell in order to produce more 'baby' viruses.

SOCIETY AND CULTURE

Humanities, Economics, Literature, Philosophy and Political Systems and Religions

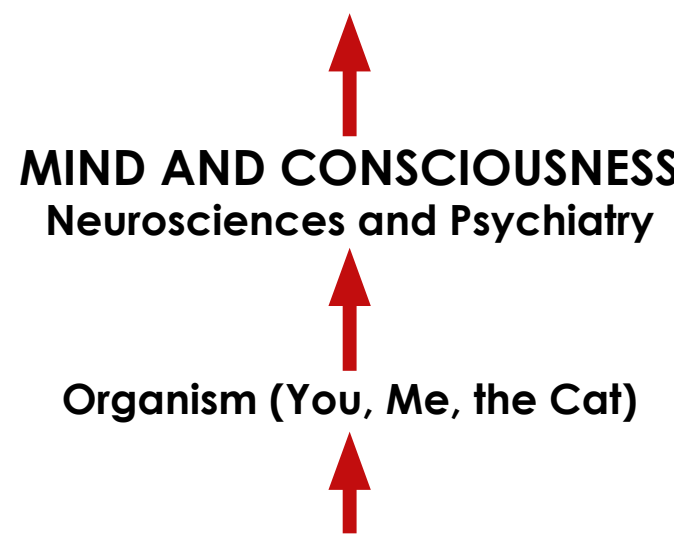

Organ Systems

(Nervous, Circulatory, GI Systems, ... etc.)

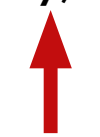

Organs (Liver, Stomach, Colon ... Brain)

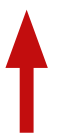

Tissues

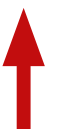

Cells - Life - Biochemistry

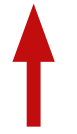

Molecules and Macromolecules

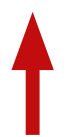

Atoms - Physics

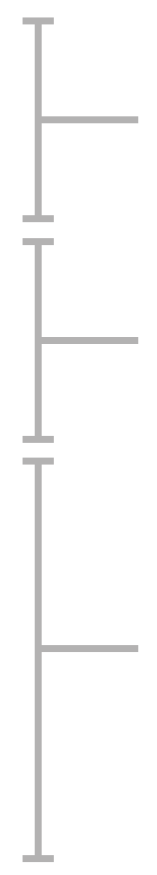

Psychology

Anatomy, Physiology

Histology
Chemistry (protein, lipids, carbohydrates, nucleic acids and vitamins) ions/trace elements, $\mathrm{H}_{2} \mathrm{O}$ 
Eventually, after another billion and a half years, more complex cells called eukaryotic cells (which have a nucleus that contains their genetic material) emerged. They are the cells that make up trees, cats, humans, and most complex forms of life on this Earth (see Figure 6.1).
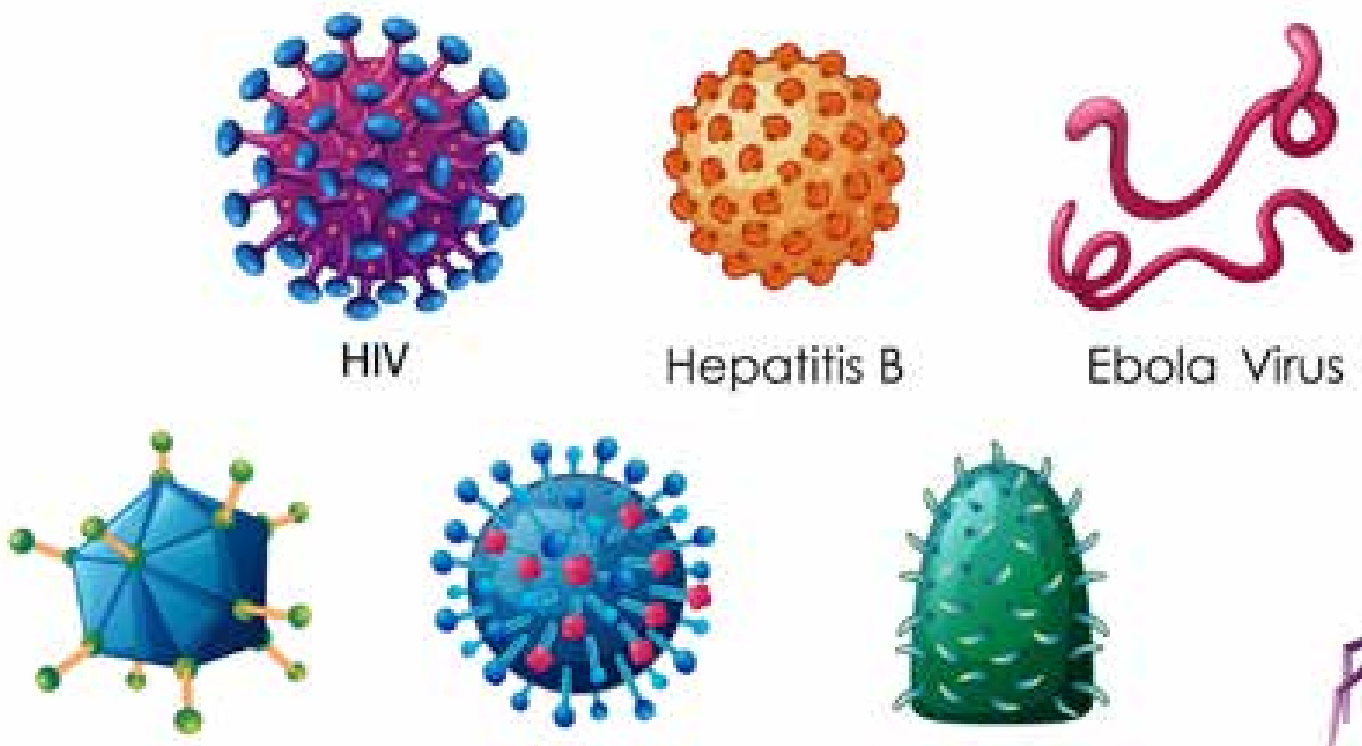

Hepatitis B

Ebola Virus

Adenovirus

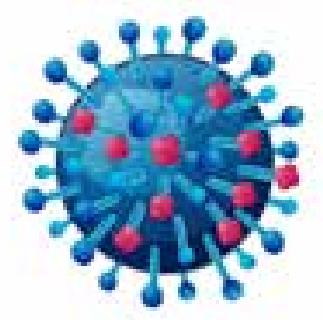

Influenza

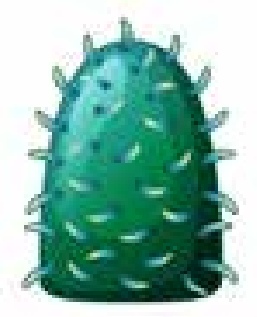

Rabies Virus

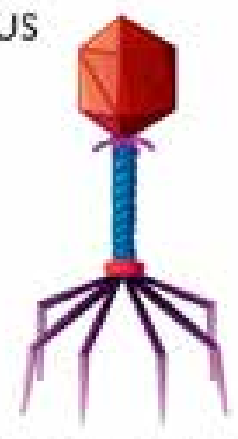

Bacteriophage

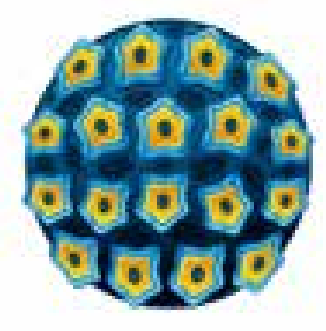

Papillomavirus

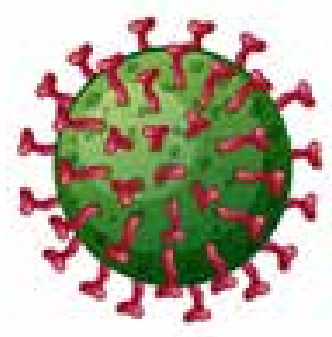

Rotavirus

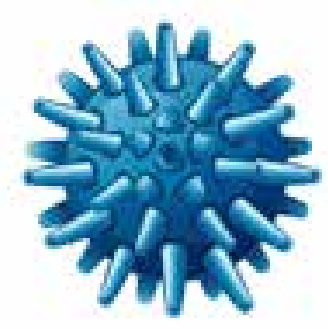

Herpes Virus

Figure 6.2: Different kinds of viruses that cause different diseases

\section{How does one define a virus?}

A virus is a particle made of a protein shell enclosing nucleic acid genetic material in the form of either RNA (ribonucleic acid) or DNA (deoxyribonucleic acid). Human disease-causing viruses cause harm by entering our bodies and replicating in our cells. Some viruses enter the nucleus of the cell and replicate in the nucleus, and a small subclass, the retroviruses, even insert their genetic material into the host DNA. Other types of viruses, like coronaviruses, replicate their genetic material in the host's cytoplasm. The virus takes over the cell and uses the cell's machinery for copying DNA or RNA to make copies of its own genetic material. During this process more viruses form. The cell can burst or, depending on the kind of virus, these new virus particles might be released in what seems to be a controlled way, thereby releasing a new population of viruses, which, in turn, can infect more cells. This is the start of 
a viral disease in the body. The individual carrying this large number of new virus particles can spread it to others (viral transmission).

There are many types of viruses (Figure 6.2), causing a variety of diseases such as HIV-AIDS.

We are interested in the coronavirus (SARS-CoV-2), which has caused the coronavirus disease pandemic (Figure 6.3).

Figure 6.3: The coronavirus with the spike proteins on its surface in red

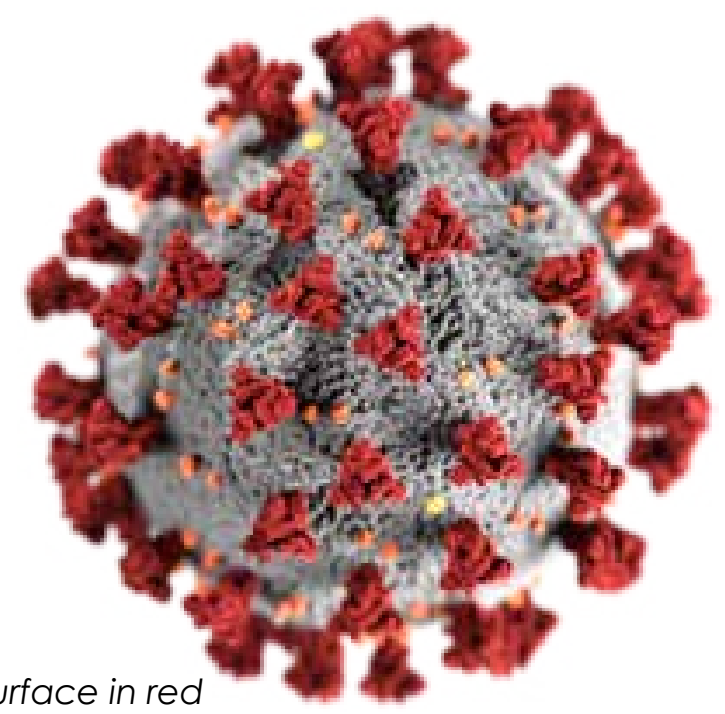

Source: Centres for Disease Control \& Prevention; https://www.cdc.gov/

US Centres for Disease Control and Prevention/Wikimedia Commons

\section{What are communicable and non-communicable diseases?}

Diseases may be divided into two categories. On the one hand, there are 'communicable' diseases, which for practical purposes are all 'infectious' diseases in which a pathogen is passed on from one organism to another (they are also known as 'transmissible' diseases.) When infectious diseases are transmitted from person to person (i.e.,between humans), they are said (by agreed convention) to be 'contagious', even though the word (which means 'arising easily from close contact') could in principle also be applied to transmission from an animal to a person, or sometimes from a person to an animal and back to a person, as in the case of malaria where the mosquito plays the role of an intermediary.

'Non-communicable' diseases (NCDs), by contrast, arise spontaneously within the body, usually as a result of environmental factors interacting with the intrinsic processes of maturation and subsequent ageing. Often, but by no means always, they also arise partly through inheritance of particular sets of genes from the mother and father. Examples are 'sugar diabetes', hypertension, and heart disease. These are called 'diseases of lifestyle' and are common in our modern societies. They are thought to be caused in part by stress, poor dietary habits, and a lack of exercise. It must be noted that no disease is due to purely environmental factors. Genes are often involved. People can contract heart problems, for example, because it runs in their families. We call this a 'familial predisposition' to heart disease. However, that does not mean that everyone with a bad family history of heart problems will have heart disease. One can avoid a 'disease of lifestyle', even though one's relatives have the disease, by leading a healthy lifestyle. 
It is important to remember in the case of infectious diseases that the condition of the host body at the time of infection plays a very significant role in determining the course and outcome of that infection.

Widespread transmission of a pathogen from a non-human animal to a human is called zoonotic in nature. If the integrity of the body has to be breached for transmission to occur, you won't catch the disease from someone you are talking to or pass on the street. An example would be Lyme disease, which is transmitted by the bite of an infected tick. Becoming infected with a newly mutated form of influenza virus, on the other hand, can happen through inhalation or ingestion of infectious materials from a slaughtered chicken or pig.

Contagious diseases (such as the flu, colds, or strep throat) spread from person to person in several ways. One way is through direct physical contact, like touching or kissing a person who is carrying the infection. Another way is when an infectious microbe travels through the air after someone nearby sneezes or coughs. Sometimes people get contagious diseases by touching or using something an infected person has touched or used, such as sharing a straw with someone who is infected. And sexually transmitted diseases (STDs) are spread through all types of sex: oral, anal, or vaginal.

You can protect yourself against contagious diseases by washing your hands well and staying away from those who are sick, keeping up to date with all vaccinations, and always using condoms during any type of sex.

\section{Vaccines against the coronavirus}

Since the early days of the coronavirus pandemic, the world was warned that there was no cure for this complex disease. Its signs and symptoms are highly variable, often reflecting a spectrum of diseased organs and systems in the body, each affected to differing degrees. Thus the overall clinical picture manifests itself in different ways. Some carry the virus but are asymptomatic-that is, they show no symptoms. Others show a variety of mild symptoms. Yet others become very ill, often, but not always, initially involving the lungs (pneumonia). There can, instead or additionally, be problems such as strokes arising from abnormal blood-clotting, kidney dysfunction, skin ailments, or gastrointestinal disorders, to name a few examples.

The only hope for eradicating the disease is the development of a vaccine against this virus. Presently, several vaccines against Covid-19 have become available, including vaccines from Astra Zeneca, Johnson and Johnson, Moderna, and PfizerBioNTech. 
When a foreign agent, or pathogen, such as a bacterium or virus-in this case the coronavirus-enters the body, the body's immune system, its 'police' and 'army', is alerted. The 'police' are a group of cells that represent the elements of innate immunity, which is non-specific in terms of the kind of pathogen they protect us against, but is capable of removing the invaders very quickly if they are present in small numbers. The 'army', which is called adaptive immunity because it is directed by the body against the specific invading pathogen, is called up more slowly over a number of days and includes two main types of 'soldiers'. One type is a cell called a B cell, which produces proteins called antibodies that bind to the surface of the foreign agent, leading to its removal from the circulatory system. Another group of 'soldiers' are the T cells. These are active 'cell-killers' that seek out the body cells that contain the virus and destroy them. In summary, the main components of the body's adaptive immune system, or 'army', against the coronavirus are the pathogen-specific $\mathrm{T}$ cells and the similarly specific antibodies secreted by the $B$ cells.

Vaccines work by training the immune system to recognise and fight off infectious agents or pathogens, such as bacteria and viruses. A vaccine often contains a slightly altered form of the pathogen. The vaccine presents to the body's immune system a part of the virus that the immune system could recognise. For

Vaccines work by presenting the immune system with a readily identifiable part of a pathogen, which the immune system remembers so that it can quickly respond should it encounter that same pathogen in the future. example, in the case of the coronavirus, this could be the spike protein found on the surface of the virus (see Figure 6.3). Other vaccines could carry a part of the coronavirus genome in the form of DNA that codes one of the virus proteins. When introduced into the body, usually as part of a harmless virus called an adenovirus, this DNA, through transcription as messenger RNA (mRNA), leads to the synthesis of the viral protein by the host cell. This causes the immune system to respond to the 'foreign' protein and to mount an immune response to it, and, thereby, against that protein on the surface of the virus. Sometimes, messenger RNA coding for the viral spike protein is used as the vaccine. Thus if in the future a person who has been vaccinated comes into contact with the actual virus, that person's immune system will quickly recognise the virus or virus-infected cell. It will mobilise the two components of its adaptive response and target both the free virus (with antibodies) and virus-infected cells (with T cells), thus offering immunity or protection to that individual against the specific infection.

In summary, vaccines work by presenting the immune system with a readily identifiable part of a pathogen, which the immune system remembers so that it can quickly respond should it encounter that same pathogen in the future. 
Once researchers are sure that they have developed a vaccine, they must select groups of people from the population to test out the safety and efficacy of the vaccine as a phased series of vaccine trials. The scientific method (described in detail in Appendix C) is used to determine whether the vaccine is safe and whether it is effective.

In the case of the Oxford vaccine, two groups were selected for this initial trial called Phase 1, each including 543 people. One group of subjects is called the 'control' group. This group is not given the real vaccine but rather another compound called a 'placebo,' designed to have no biological effect. The second group is the 'experimental' group. This group is given the real vaccine. The trial is analysed by looking at differences between the two groups. None of the subjects knows whether they have been given the actual vaccine or the placebo. This comparison removes a lot of possible errors, including the 'placebo effect', a psychological effect where people given a fake drug falsely believe to have benefited.

Before testing a vaccine in humans, researchers also first test the same vaccine candidate on mice and rhesus macaque monkeys. These experiments have shown that the vaccine is probably safe and able to induce a strong immune response. In fact, a far higher dose of the coronavirus was used in the animal models than humans got through infection, but the vaccine still protected them from severe disease. 


\section{CHAPTER 7}

\section{Medical Aspects of Covid-19}

Coronavirus (SARS-CoV-2-an RNA virus) is found in birds and mammals, with bats being a rich source of the virus. Our current understanding is that the transmission of the coronavirus from bats to humans occurred via another intermediate animal host, by what is known as a zoonotic transfer. at is spread quite easily by the expulsion of droplets when an infected person coughs or sneezes. Those infected and showing symptoms have Covid-19 disease, which can be mild with a quick recovery, or severe, requiring intensive care treatment. The disease is diagnosed by a viral test using nasal swabs or a blood test (serology) that shows if the body has producedantibodies against the disease. Not everybody has symptoms - they can be asymptomatic and still spread the virus. To avoid spreading the virus, immediate self-isolation is advised. Most vulnerable are the aged and those with co-morbidities such as diabetes, hypertension and heart disease. In cases of severe disease, the virus is known to attack many systems and organs in the body. The virus survives on different surfaces for varying periods and, since there is no cure. prevention is necessary by social distancing, regular handwashing and the wearing of a mask, as well as self-isolation when one has come info contact with a known infected person or if one feels symptoms.
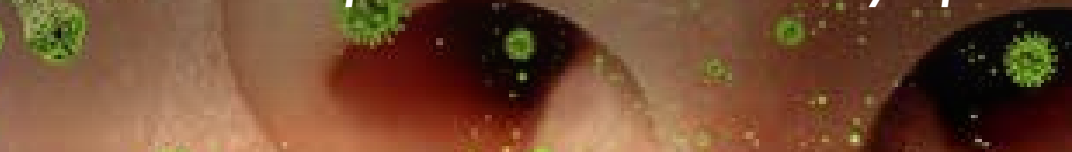

6.

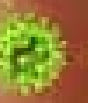

$3 x+45$

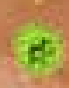




\section{SARS -CoV-2 nomenclature}

Virus: SARS-CoV-2 (Severe acute respiratory syndrome-related to coronavirus 2)

Disease: Covid-19 (coronavirus disease 2019)

\section{What we know about coronaviruses and humans?}

Coronaviruses, first discovered in the 1960s, are found in birds and mammals, especially in bats, civets, camels, and rats. The causative agent of Covid-19 (SARS-CoV-2) belongs to the genus Coronavirus, family Coronaviridae, and order Nidovirales. SARS-CoV-2 is the 7th coronavirus known to infect humans. Notwithstanding restricted geospatial transmission, SARS-CoV (Asia) in 2003 and MERS-CoV (Middle East) in 2012 caused severe disease. In contrast, coronaviruses HKUI, NL63, OC43 and $229 \mathrm{E}$ are associated with mild symptoms and represent the cause of about a fifth of 'common colds' in most parts of the world.

Bats are a rich source of coronaviruses, although only a few of these coronaviruses can infect humans. SARS and MERS viruses have zoonotic transmission, originating from bats and passing through palm civets and camels, respectively, as the intermediate hosts. The most recent reports have suggested that SARS-CoV-2 is a modified coronavirus of bat origin, which came to humans as a result of zoonotic transmission by first adapting to an intermediary host.

\section{Origin and characteristics of SARS CoV-2}

The true origin of SARS-COV-2 in humans remains to be established. Phylogenetic analysis (the study of evolutionary relationships among biological entities-often species, individuals or genes-where viruses isolated from humans and or other animal species are sequenced and compared to identify common ancestry or where differences exist between species) suggests that bats are the original host, while an animal sold at the seafood market in Wuhan might be an intermediate host facilitating the emergence of the virus in humans.

Phylogenetic analyses show that the pangolin coronavirus is genetically related to the SARS-CoV-2 and other bat coronaviruses. It carries the same receptor binding domain as does SARS-CoV-2 but the rest of the virus is only $90 \%$ genetically similar. Pangolins may therefore not be the intermediary host. SARS-CoV-2 binds with high affinity to human Angiotensin-converting enzyme 2 (ACE-2) as its cellular receptor and uses it as an entry point to invade target cells in humans. ACE-2 receptor expression is much higher in the lower lobes of our lungs compared with the upper respiratory tracts. 


\section{Is the virus likely to mutate into forms that are more or less dangerous?}

SARS-CoV-2 is a large RNA virus and appears to have an effective repair system when errors are made in copying its single RNA strand during replication in infected host cells. Most of the mutations since the start of the pandemic have not drastically changed the properties of the virus, although there have been plenty of 'neutral' mutations that have been detected by whole-genome sequencing of samples from all over the world. Analysis of these is a fast-developing way of tracking the source of particular outbreaks and responding appropriately to them. However in the past year a few variants that spread more readily have become a major cause of concern.

Bilateral multi-lobular lung involvement is common in Covid-19, but we now know that it is a multi-organ disease affecting human beings from head to toes. Not everyone who gets infected gets sick. In fact, the majority remain asymptomatic. Not everyone who gets ill develops Acute Respiratory Distress Syndrome (ARDS) requiring mechanical ventilation.

Respiratory droplets and aerosols transmit the virus through exhalation, such as sneezing, coughing, singing, and talking. There is much evidence to suggest that the virus is air-borne in aerosols, which remain in the air much longer and can migrate further than virus contained in the much larger droplets that fall quickly onto surfaces like the ground or floor.

\section{Does everyone who gets exposed to SARS-CoV-2 get the disease?}

Not everyone exposed to SARS-CoV-2 develops the disease. Those at greatest risk of infection are persons who have had prolonged, unprotected close contact (i.e., within 2 metres for 15 minutes or longer) with a person with 'active' (confirmed or unconfirmed) SARS-CoV-2 infection. The virus is spread by infected persons regardless of whether the person has symptoms or not. In fact, the peak concentration of the virus in an infected person is just before the onset of symptoms.

While everyone exposed to SARS-CoV-2 appears to be at some risk of acquiring the infection, the severity of the resulting clinical disease differs markedly by age. According to the Chinese Centre for Disease Control and Prevention, which analysed more than 44,672 confirmed cases, age is the most important predictor of severe disease and death. The case fatality rate was $<1 \%$ among $<60$ years old; $3.6 \%$ for those $60-69$ years; $8.0 \%$ in people $70-79$ years; and $14.8 \%$ among those 80 years or older. 


\section{What is the course of a Covid-19 infection?}

The time from exposure to development of symptoms for SARS-CoV-2 infection ranges from 2-14 days with an average incubation time of 4-5 days.

\section{Common presenting symptoms of the disease:}

- fever (88\%)

- cough (68\%, 33\% productive)

- fatigue (38\%)

- shortness of breath (dyspnoea)(19\%)

- sore throat (14\%)

- muscle pain (myalgia) (15\%)

- loss of sense of smell (anosmia) (variable but frequent)

- loss of sense of taste (ageusia) (variable, less frequent)

- severe headaches (14\%)

- nasal congestion (5\%)

- diarrhoea (4\%)

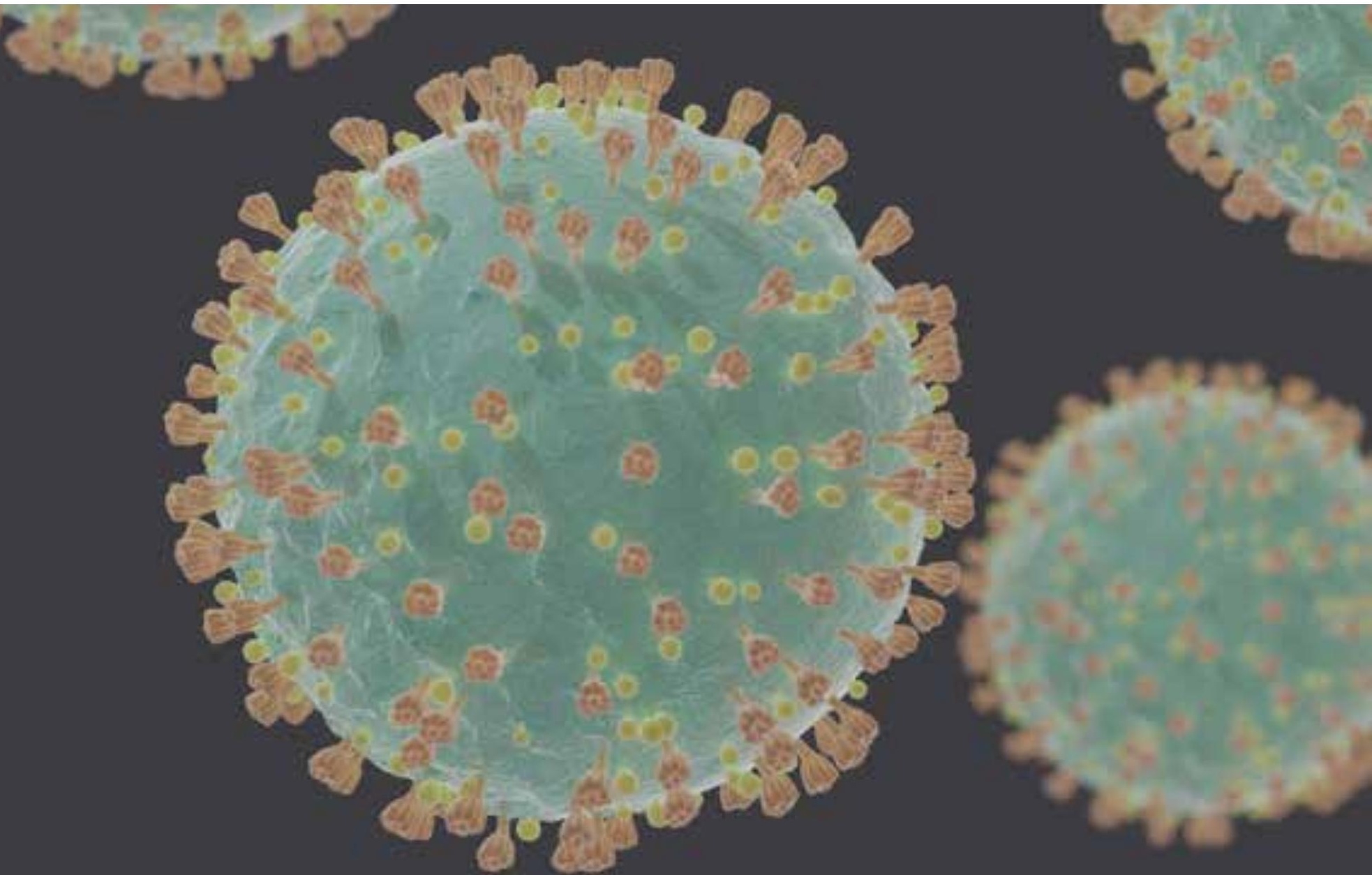




\section{COVID - 19 General Symptoms}

Recognised symptoms of the new disease continue to be updated as studies are published.

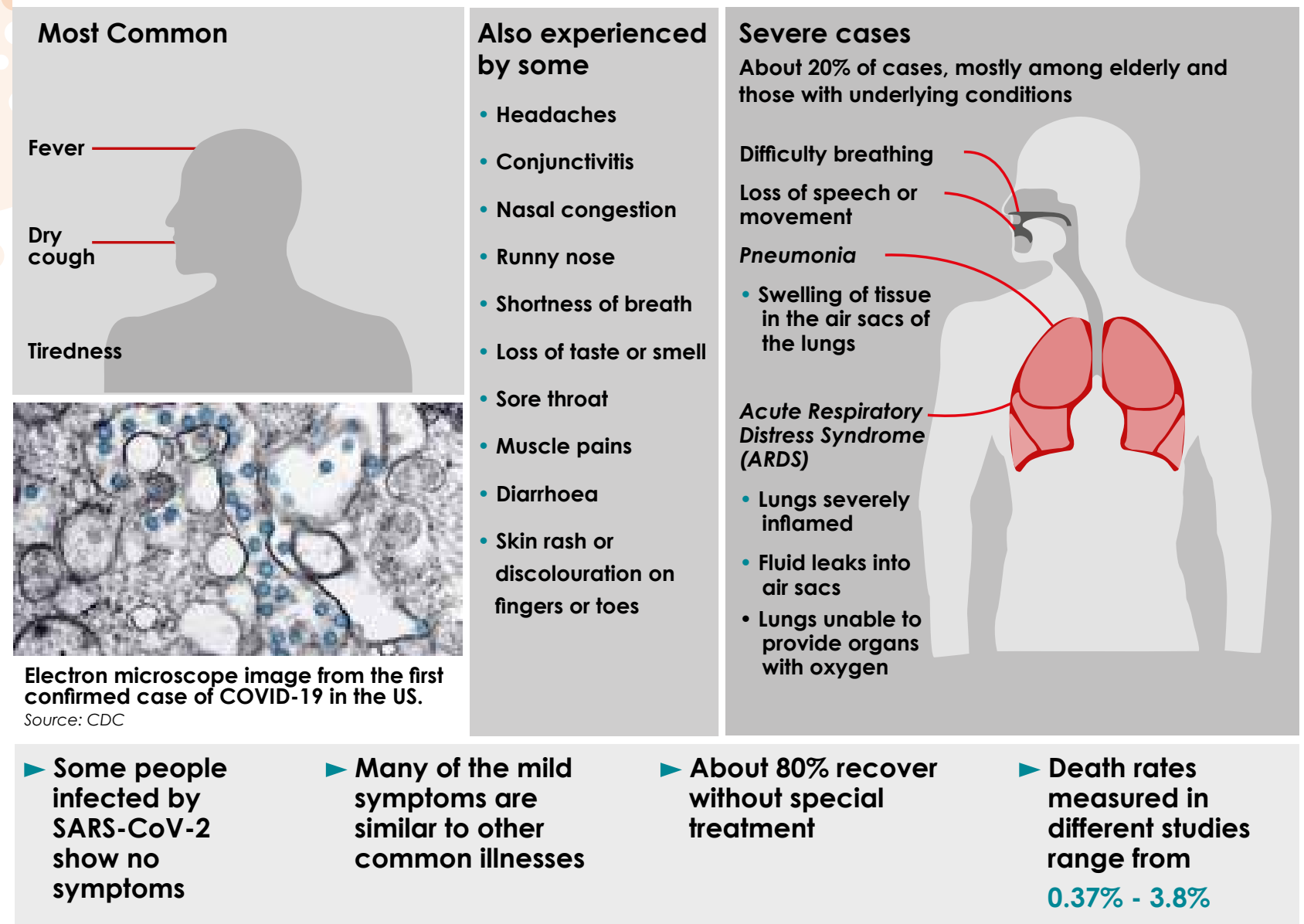

Figure 7.1: Covid-19 general symptoms

Source: WHO/CDC

An asymptomatic infection-a person infected with Covid-19 who does not develop any symptoms.

Symptomatic infection-these symptoms can range from mild to critical. Most people (about 80 per cent) recover from the disease without needing special treatment.

Around one out of every six people who gets symptomatic Covid-19 becomes seriously ill and develops difficulty breathing.

\section{Severity of disease presentation: (WHO clinical guidelines of grading)}

Mild-fever, cough, fatigue, anorexia, shortness of breath, myalgia. Other nonspecific symptoms, such as sore throat, nasal congestion, headache, diarrhoea, nausea and vomiting, anosmia and ageusia.

Moderate-Above symptoms with clinical signs of pneumonia and $\mathrm{SpO}_{2}>90 \%$

Severe-Respiratory distress with $\mathrm{SpO}_{2}<90 \%$

Critical-Acute Respiratory Distress Syndrome (ARDS) 


\section{Coronavirus progression in majority of cases}

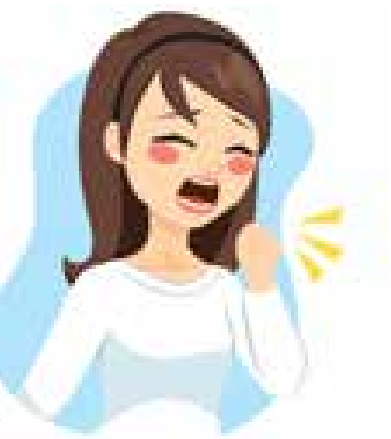

\section{Exposure to the virus}

Incubation period

4 to 6 days (average)

Can be up to 14 days

Person develops symptoms

Illness 10 days

But can be longer
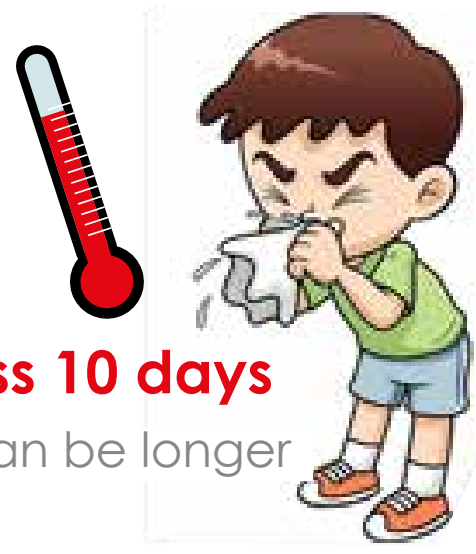

No more symptoms

3 days
Infectious period 8 to 10 days

(but can be longer)

Starts from 1-3 days before symptoms develop

\section{Released from isolation}

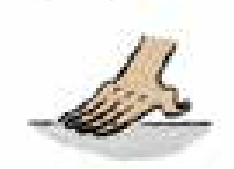




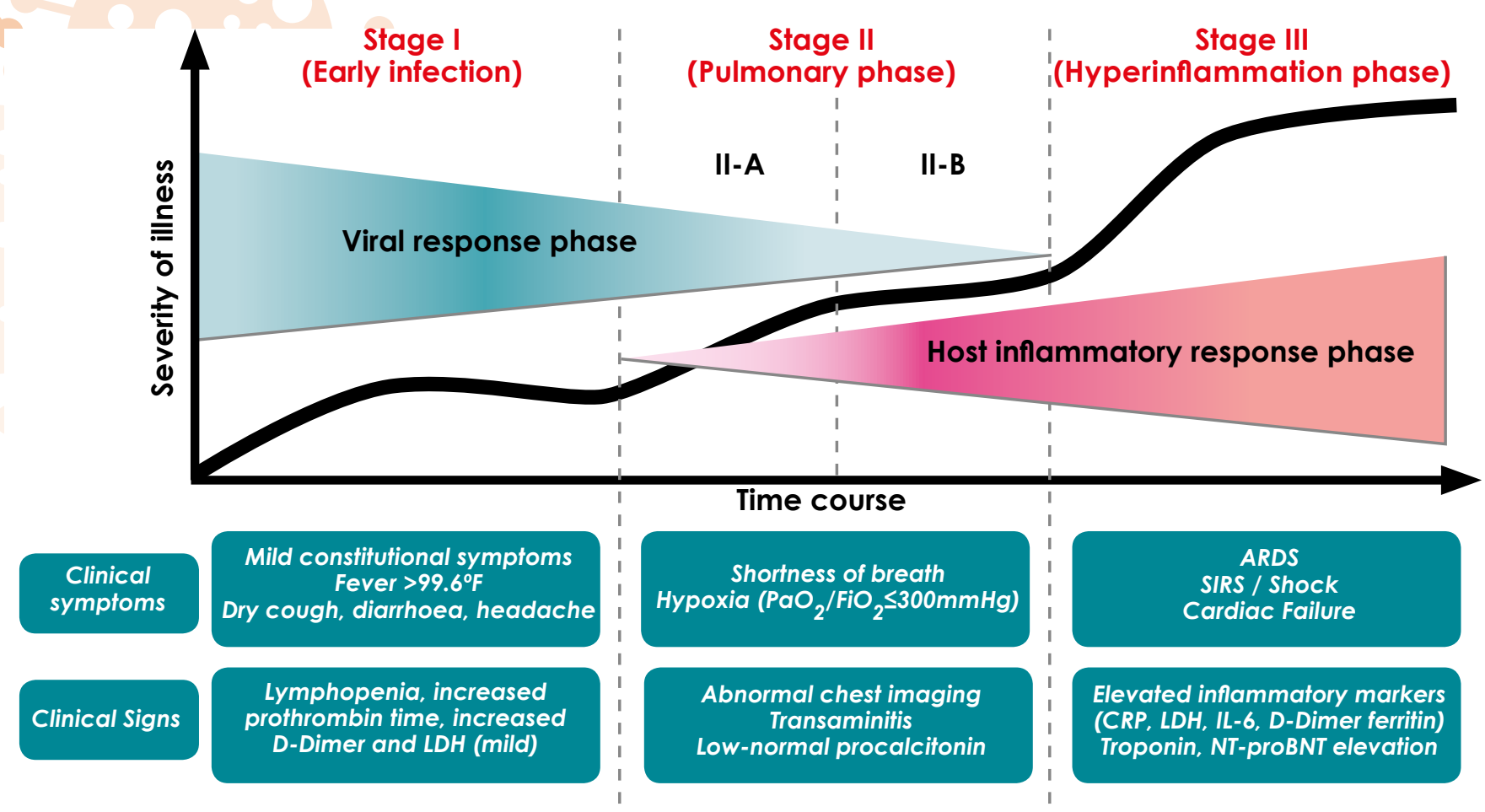

Figure 7.3: Schematic representation of the natural history of Covid-19 from the onset to recovery or death

Source: US NIH

There are basically three stages or phases in the natural history of Covid-19, regarding disease severity. The first phase is related to the onset of the disease and is generally characterised by the development of influenza-like symptoms from mild to moderate. Some individuals recover and some progress to the second phase. Depending on the severity of phase 2, patients can improve or worsen with the necessity of intubation and ventilation. Phase 3 is characterised by hyper-inflammation and sepsis of lungs. Patients often require intensive care and most of them, unfortunately, cannot overcome the infection and may eventually die.

\section{What is the spectrum of disease?}

SARS-CoV-2 affects different organ systems at different times. There is a growing body of evidence suggesting that Covid-19 is a multi-system disease, with the causative virus capable of infecting and affecting any organ in which its receptor is present. The spikes on the surface of SARS-CoV-2 virus particles each comprise three identical subunits, each of which, in turn, is made up of two functional components; the S1 part is responsible for binding to the host cell receptor and the S2 part is for the fusion of the viral and cellular membranes. Angiotensin converting enzyme 2 (ACE2) embedded in the surface membrane of many cells is the functional receptor for SARS-COV-2 to gain access to human cells. The ACE2 receptor is highly expressed in lung epithelial cells, kidneys, gastrointestinal tract, liver, vascular endothelial cells, and arterial smooth muscle cells. Thus, all of these organs and systems with high expression of ACE2 receptors are targets for SARS-CoV-2 infection. Covid-19 may induce a widespread inflammatory response involving all these organs. 
Multi-system Involvement of Covid-19: A disease from head to toe

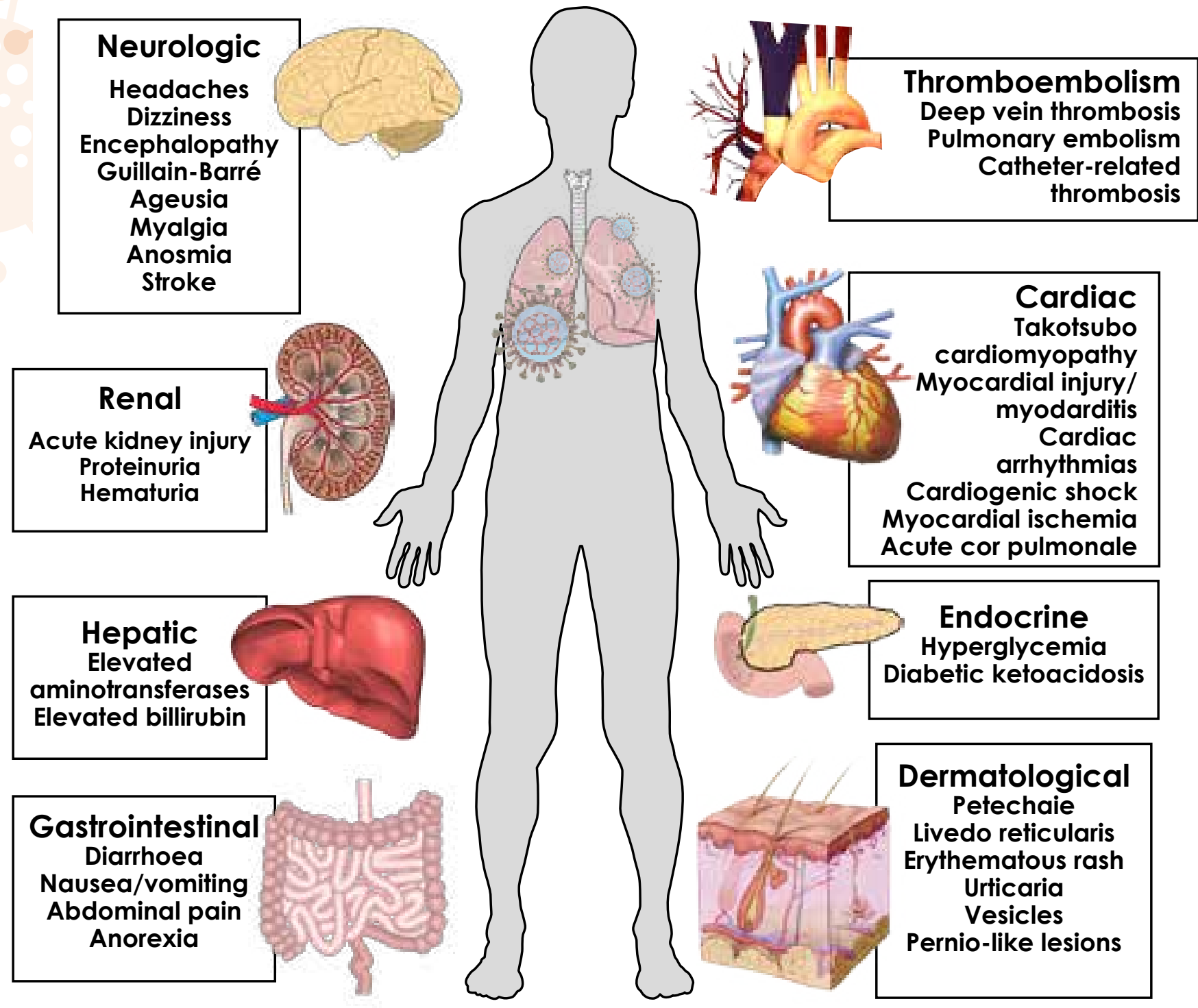

Figure 7.4: Multi-system involvement of Covid-19: A disease from head to toe

\section{Why does Covid-19 make so many people sick?}

- It is an emerging infectious disease, which means the human population has not previously been infected and, therefore, people do not have immunity to the virus.

- The virus spreads rapidly and easily.

- Each person's immune system must apply all its components to try to find the right defences to protect the body. This takes from hours to days to weeks, during which time the virus continues to replicate and spread.

- The majority of people infected with the virus don't develop any symptoms but are still infectious i.e. they continue to spread the virus.

- About $80 \%$ of infections are transmitted by 'super-spreaders', who are infected individuals who might shed more virus into the environment than others if their 
immune system has trouble subduing the invader. (We now know that some examples of 'super-spreading' are not so much due to the infectiousness of the infecting person, but the circumstances in which large numbers of other people are exposed to an infected person, e.g., a large number of party-goers crowded together in an indoor venue, laughing and singing and not wearing masks.)

\section{How does Covid-19 spread from person to person? Droplets, aerosols, and surfaces?}

Transmission in most instances takes place via inhalation or ingestion of respiratory droplets (generated through coughing and sneezing) or the much smaller and lighter respiratory aerosols (generated by singing or talking loudly or breathing heavily during exercise), and by direct transfer of virus particles from contaminated surfaces to the mouth, nose or eyes.

The stability of virus in air and on various surfaces is: Air; Copper ( 4 hours); Cardboard (24 hours); Stainless Steel (48 hours); Plastic (72 hours).

Droplet or aerosol transmission usually occurs when a person is in close contact (within 1 metre) for longer than a few minutes with someone who has active virus, whether symptomatic or not. It can also occur by direct contact with still-active virus left on surfaces in the immediate environment of, or on objects used by, actively infected persons (e.g., stethoscope or thermometer).

In hospitals or ICUs, airborne transmission by droplets or aerosols is especially likely in specific circumstances and settings in which procedures or support treatments

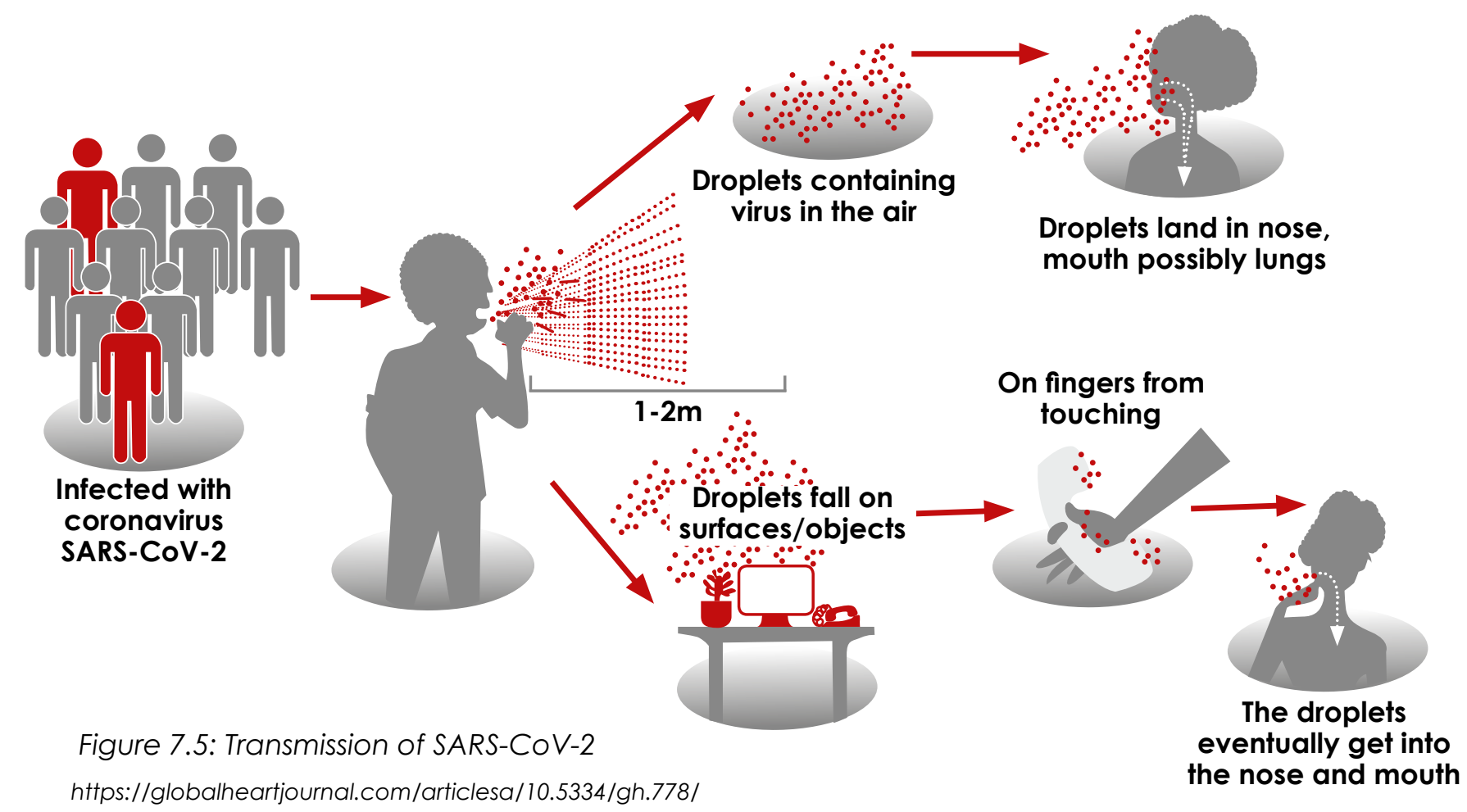


that generate aerosols are performed; i.e.,endotracheal intubation, bronchoscopy, open suctioning, administration of nebulised treatment, manual ventilation before intubation.

\section{Some general observations:}

- The virus is usually detectable in the nasopharynx/oropharynx 1-3 days before the onset of symptoms.

- The viral load peaks around the time of symptom onset.

- Viral loads are generally higher in sputum samples than in nasopharyngeal or oro-pharyngeal swabs.

- The virus is detectable by PCR in the nasopharynx/oropharynx for $\geq 7$ days after the onset of illness in most cases, but culturable virus seems to be rare after day 7 .

- Pre-symptomatic transmission is an important contributor to community transmission (wide range of estimates-up to $50 \%$ ).

- Transmission occurs from symptomatic cases in the first few days of symptoms.

- Transmission after 7 days of illness is likely to be rare.

\section{Why is it so difficult to stop the spread of the virus?}

Pre-symptomatic infectiousness-people spreading it, without knowing it, before they get ill.

Asymptomatic infectiousness-people spreading it without ever knowing they have the infection.

Rapid spread-infected person infects more than one other person (Chapter 5).

Super-spreaders-such as patient 31 in South Korea who infected over 1000 people in a few days.

Repeated waves/resurgence-even when initial epidemic is controlled, there is an ever-present risk of new epidemics.

Emergence of new variants-that are able to escape natural or vaccine induced immune responses.

\section{What makes SARS-CoV-2 infection so serious?}

Case fatality rates (how many people die from the disease) are not high, but.... The virus has a genetic advantage in the form of the spike protein's high affinity for a widely distributed cell-surface receptor.

The virus spreads easily and remains viable outside the body for several hours or days depending on the way it leaves and the material of any surfaces it lands on. 
Stopping spread is complicated as the virus is highly infectious and spreads even before an infected person has symptoms (pre-symptomatic spread) and, at the same time, the majority of exposed and infected individuals remain asymptomatic. The transmission rate is therefore high-on average, one person infects between two and three others; but, in the circumstances in which spreading is most effective (closed, unventilated spaces with many people not wearing masks and talking loudly or singing, etc.), super-spreading can occur with tens or hundreds of people being infected in a single evening. It is estimated that about $80 \%$ of transmissions take place at such super-spreading events from only $<10 \%$ of infected individuals. Even when substantial reductions in new infections have been achieved, there can readily be a resurgence/second wave of infection.

The virus replicates and spreads rapidly within a matter of days. This rapid spread and surge of infections can easily overwhelm health services, resulting in higher death rates than would be the case if all patients received optimal treatment. Greater vulnerability with increasing age, and the presence of co-morbidities. During winter it is challenging to differentiate between flu and Covid-19.

\section{What should one do if one has symptoms consistent with Covid-19?}

Once persons suspect that they may have Covid-19, they should:

- Self-isolate: Self-isolating means staying at home and staying away from other family members to avoid spreading the virus. Ideally, people who have symptoms of Covid-19 should remain in one room and use a separate bathroom to other members of the household. Self-isolation is appropriate for those with mild symptoms.

- Seek medical help if needed: A person should contact a doctor by phone to report their symptoms and seek further advice and should not show up at a doctor's office without prior approval from the facility. People should call the emergency services if their symptoms are severe or include breathing difficulties.

\section{Who is most at risk? Comorbidities, morbidity, and mortality.}

While cases of Covid-19 have only recently been detected in countries with substantial HIV epidemics, there are no data available on whether the virus will exacerbate HIV infection or how HIV infection may impact the SARS-CoV-2 virus. Data from other respiratory illnesses, such as influenza, suggest that people with compromised immunity, such as those living with HIV, particularly those with low CD4 T-cell counts, are likely to be at higher risk of developing severe Covid-19 illness. In countries like South Africa that have generalised HIV epidemics and an already overburdened health care system, the impact of Covid-19 is likely to put severe strain on the services. 
Other predictors of disease progression include malnutrition (both under-weight and overweight/obesity) and non-communicable diseases (NCDs), including hypertension and diabetes. Malnutrition and NCDs are prevalent in South Africa and have been associated with higher risk and severe outcomes related to other influenza-like illnesses.

Older age (being over 60 years) and pre-existing medical conditions (diabetes; hypertension; HIV; TB; cardiovascular disease; cancer patients) appear to be important factors for severe illness and death. There is growing evidence that Covid-19 can also itself either cause or exacerbate metabolic syndrome (prediabetic condition) and hypertension.

\section{How can doctors help patients with serious Covid-19?}

Not all patients with Covid-19 will require medical supportive care or hospital admission.

Clinical management for hospitalised patients with Covid-19 is focused on supportive care for complications, including supplemental oxygen and advanced organ support for respiratory failure, septic shock, and multi-organ failure.

\section{What is acute respiratory distress syndrome (ARDS)? How is it treated?}

The majority of mortality attributable to SARS-CoV-2 infection occurs through the development of viral pneumonia-induced acute respiratory distress syndrome (ARDS). While the exact mechanisms through which SARS-CoV-2 causes ARDS and how certain host factors confer an increased risk of developing severe disease remain unclear, one factor has emerged as a dominant predictor of disease severity and risk of mortality: age.

This is an inflammatory process which can lead to insults to the lung, either direct or indirect. 


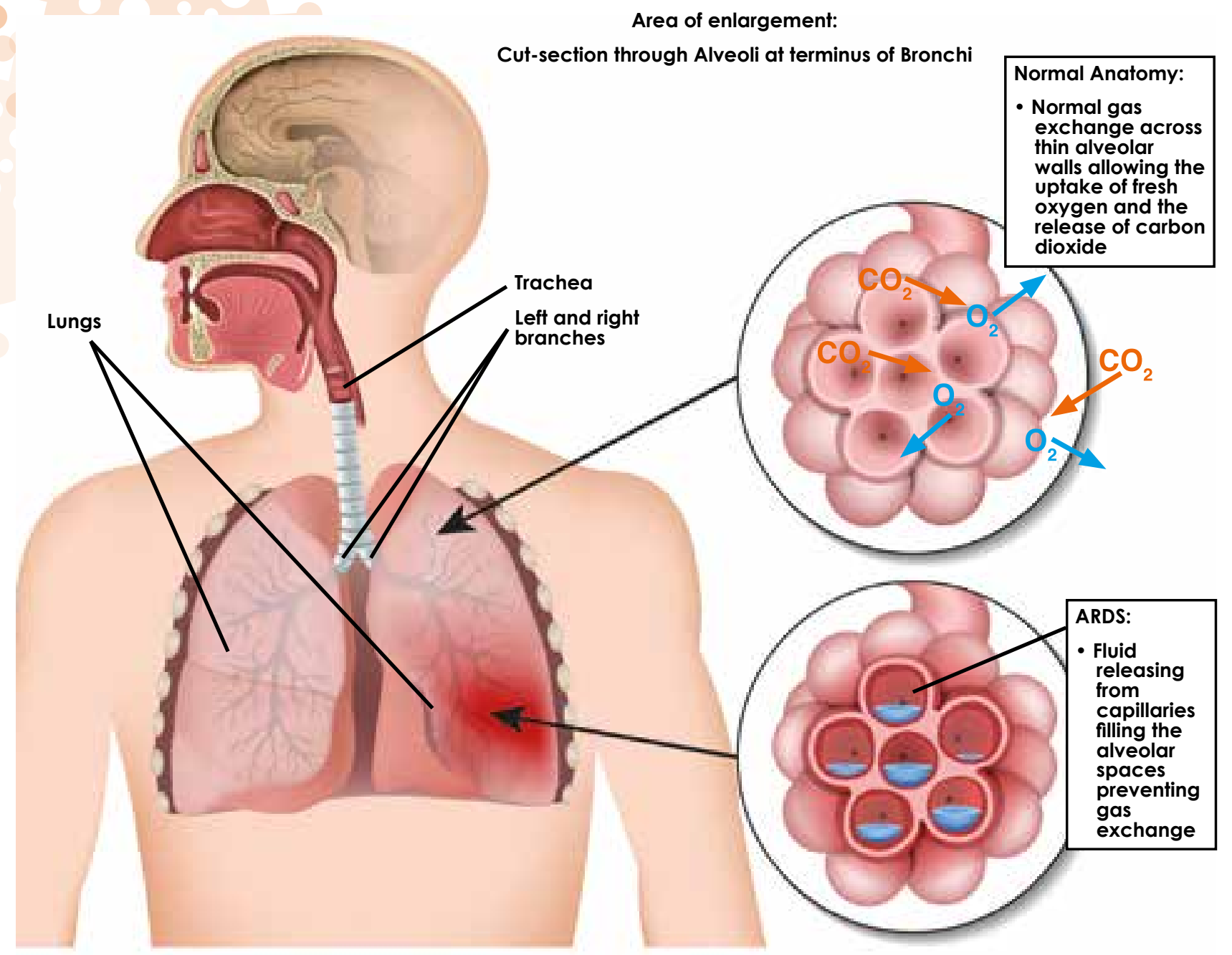

Figure 7.6

Figure 7.6: The early exudative stage presents diffuse alveolar damage with destruction of epithelial and endothelial cells. ARDS is characterized by an increase in the permeability of the alveolar-capillary barrier, leading to an influx of fluid into the alveoli. Fluid builds up in the tiny, elastic air sacs (alveoli) in your lungs. The fluid keeps your lungs from filling with enough air, which means less oxygen reaches your bloodstream. This deprives your organs of the oxygen they need to function.

Patients who have severe difficulty breathing and require oxygen may need mechanical ventilation. 


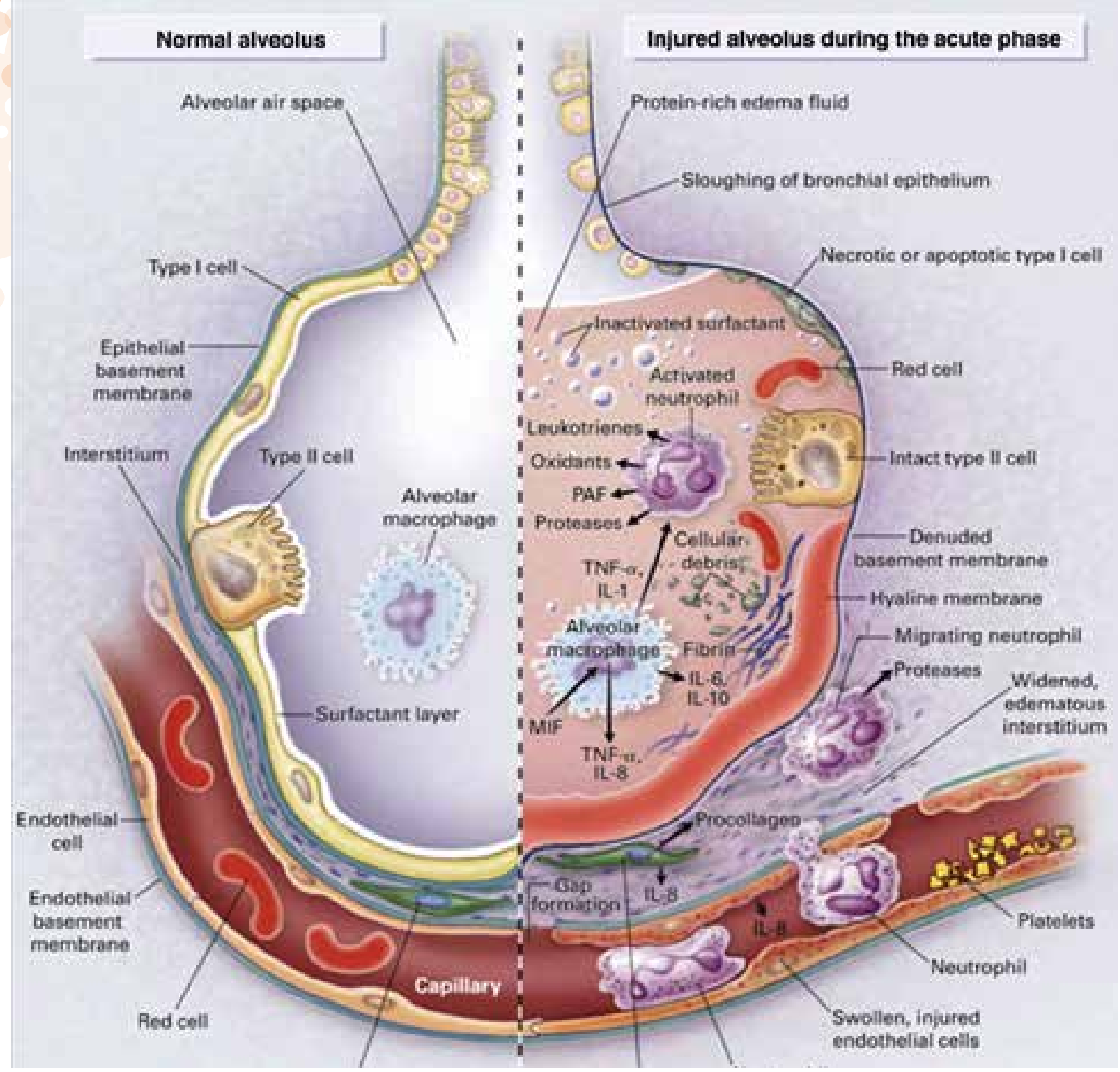

Figure 7.7 Formation of protein-rich pulmonary edema fluid. Normal and injured alveolar-capillary barriers are illustrated in left and right panels

Reprinted with permission: SM Vogel et al., Comprehensive Physiology 2 (2012) 449

About 5-15\% of patients with Covid-19 infection require intensive care surveillance and ventilatory support, mainly for one reason: severe hypoxemia (an abnormally low concentration of oxygen in the blood). The goal of treatment should be maintenance of oxygen saturation $>90 \%$. That means supplementation of oxygen via several ways.

There are no clear evidence-based guidelines for the ideal time to proceed to mechanical ventilation in patients with Covid-19. Availability of ventilators, intensive care capacity, considerations of palliative care and end-of-life resources as well as individual patient characteristics all play a role in decisions to institute mechanical ventilation. 


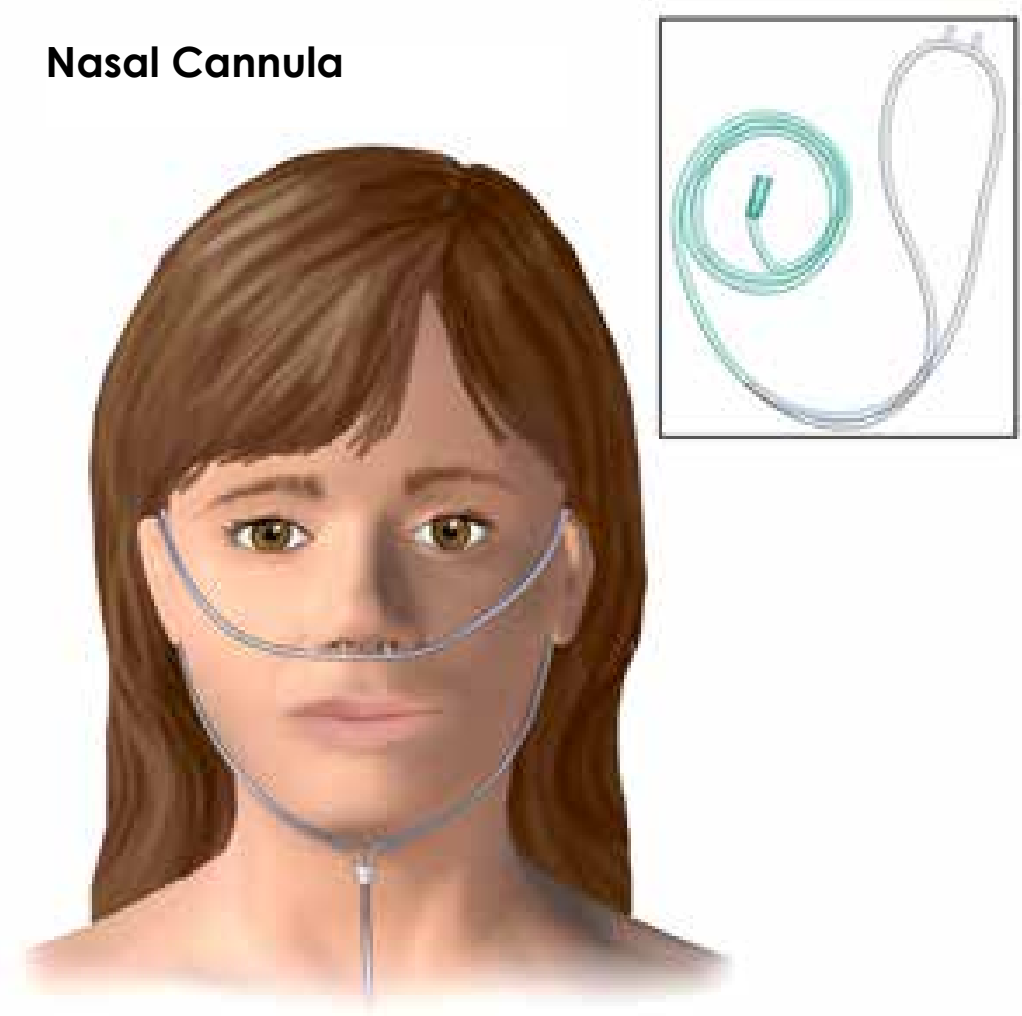

- Effective for mildly hypoxic patients, supplemental oxygen delivered by nasal cannula can induce significant dispersion of exhaled air, even at low flow rates.

- Supplemental oxygen by nasal cannula provides up to about 5-6 L/min

\section{Simple Face Mask}

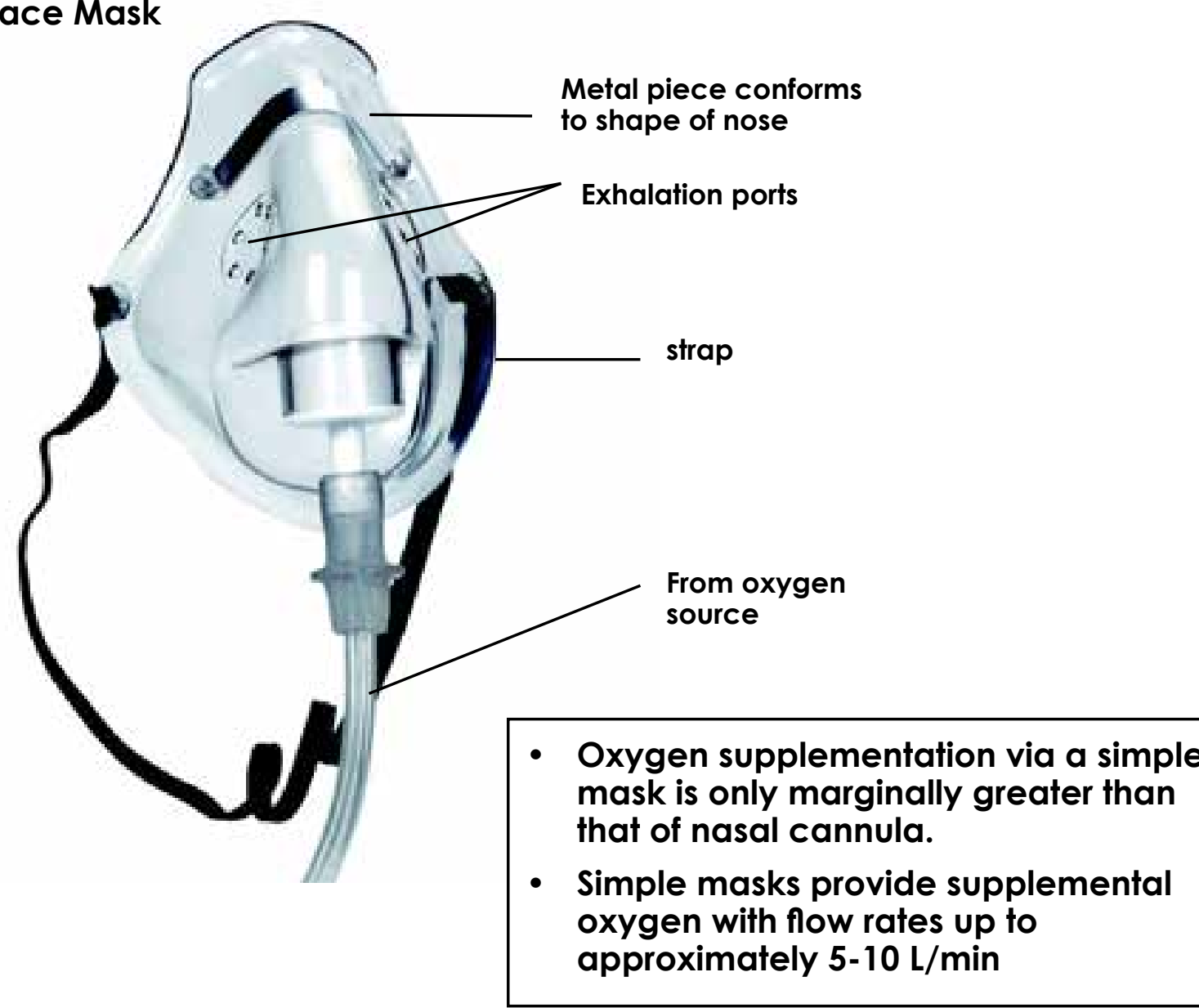


High Flow Oxygen System

flow-meter

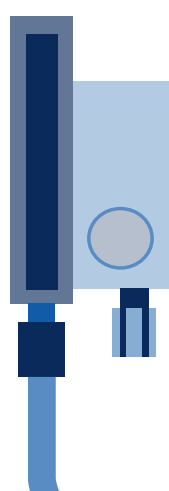

nasal cannula

air-oxygen blender

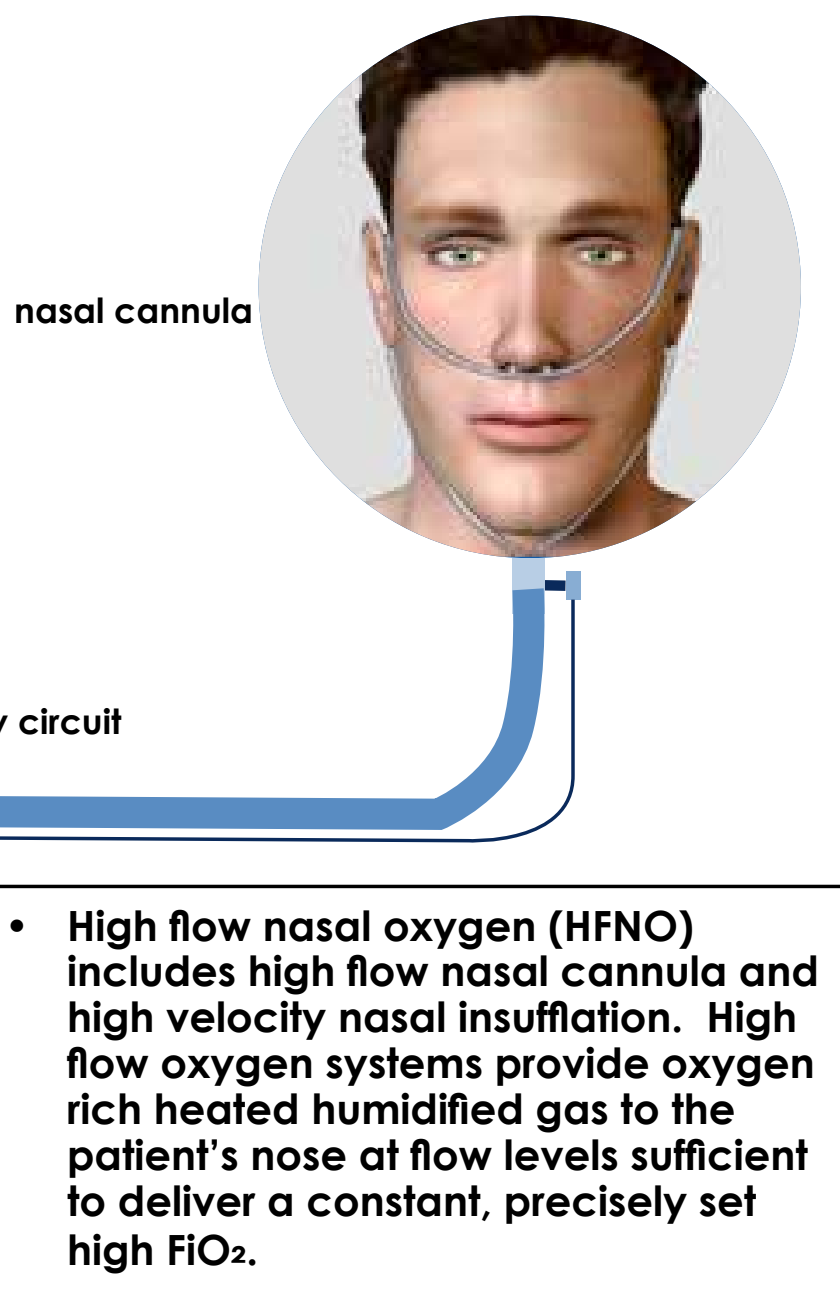

Continuous positive airway pressure (CPAP)

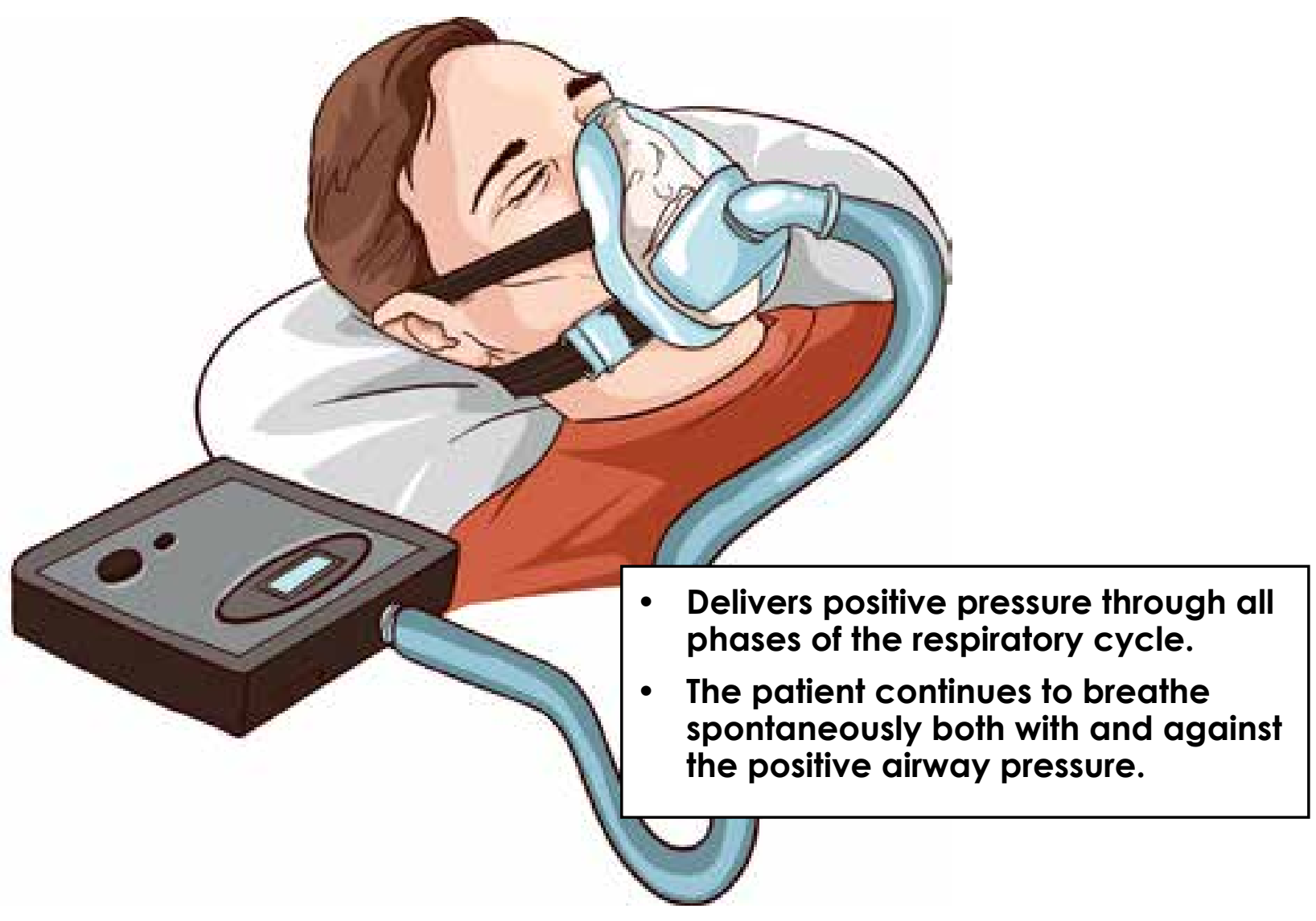




\section{Mechanical ventilation}

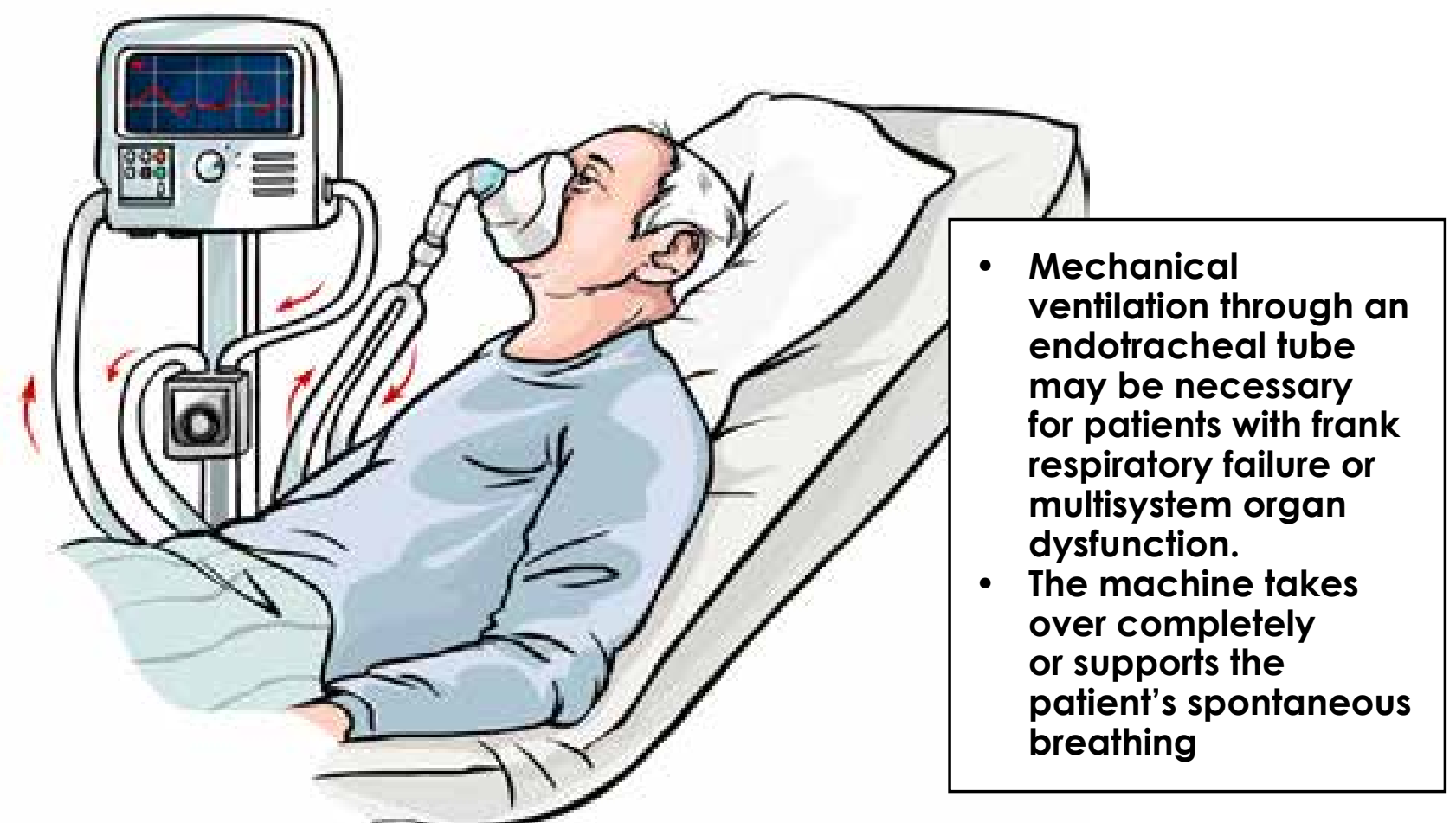

Figure 7.8

\section{Clotting and strokes}

Strokes occur when the brain's blood supply is interrupted, usually by a blood clot. Covid-19 causes complications to younger patients being hospitalised for, and sometimes dying from, serious strokes. It is unknown if coronavirus itself stimulates blood clots to form, or if they are a result of an over-active immune response to the virus.

These strokes are found in patients who test positive for coronavirus but who do not have any traditional risk factors for stroke. They tend to have no Covid-19 symptoms, or only mild symptoms. The type of stroke occurring in these patients typically occurs in much older patients.

Covid-related strokes occur because of a body wide increase in blood clot formation, which can damage any organ, not just the brain. A blood clot in the lungs is called pulmonary embolism and can cause shortness of breath, chest pain, or death. A blood clot in or near the heart can cause a heart attack. Blood clots in the kidneys can cause kidney damage requiring dialysis. 


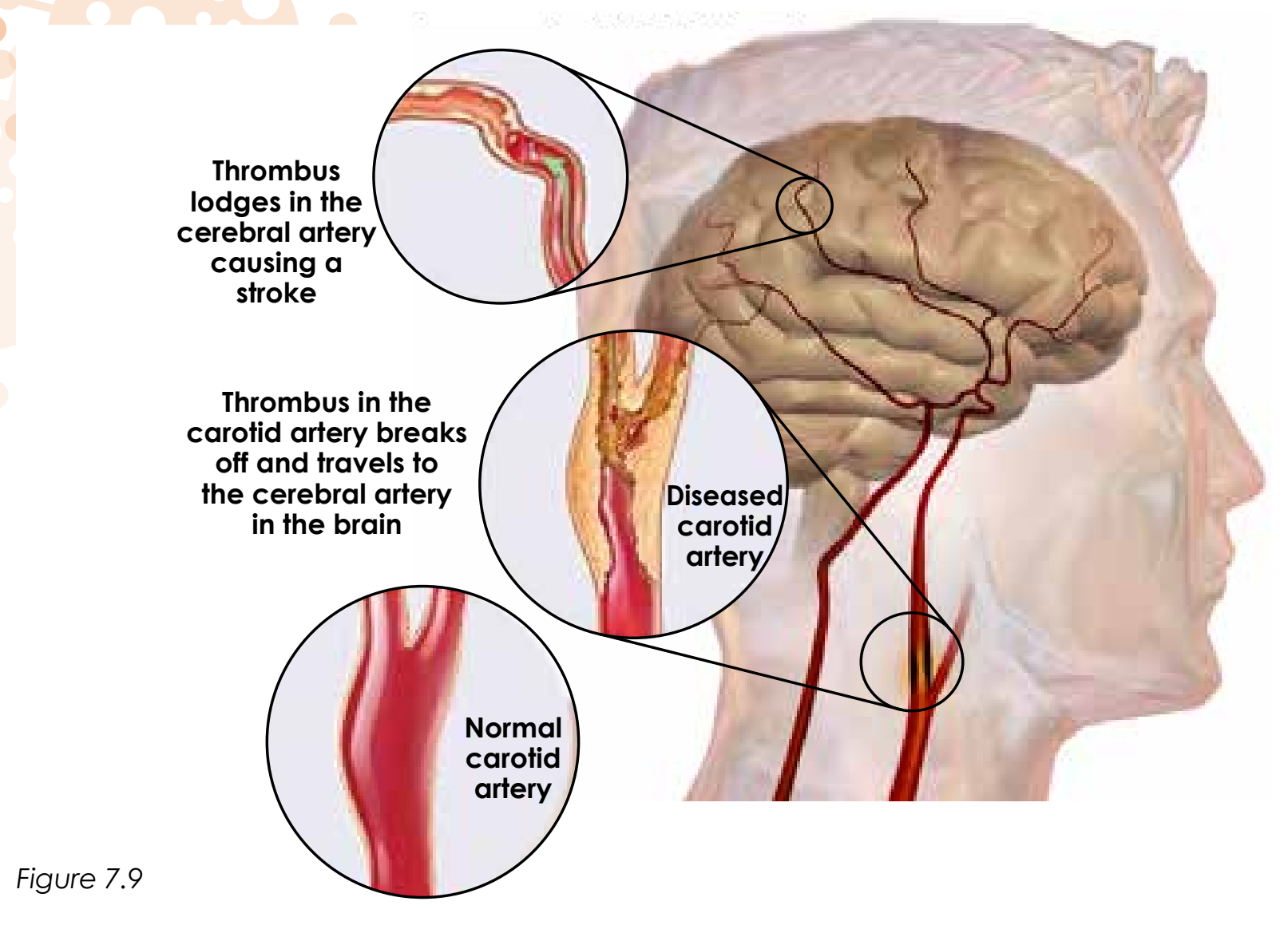

How does one minimise the risk of spread of Covid-19?

The best measures are directly relatable to the ways in which SARS-CoV-2 is spread. As already described, Covid-19 is transmitted through inhalation or ingestion of respiratory droplets and/or aerosols emitted by an infected person (respectively generated through coughing and sneezing, and talking or singing or breathing heavily). Individuals can also be infected from touching surfaces contaminated with the virus and then touching their faces (e.g., eyes, nose, mouth).

As with other respiratory infections like the flu or the common cold, public health measures are critical to slow the spread of illnesses. Public health measures are everyday preventive actions that include use of non-pharmaceutical interventions:

- Staying home when sick;

- Covering mouth and nose with flexed elbow or tissue when coughing or sneezing;

- Disposing of used tissues immediately;

- Washing hands often with soap and water or sanitiser for at least 20 seconds;

- Cleaning frequently touched surfaces and objects;

- Using face barriers such as layered cloth masks or medical masks;

- Maintaining social distance (>2 metres)- an extreme form of social distance is a national lockdown; other forms include stay at home/remote working;

- Using personal protective equipment (PPE) in the case of all health care workers (respirators; surgical masks; face shields; goggles; gloves; plastic gowns or aprons) 


\section{How does one test for Covid-19 and what do the tests tell you?}

There are currently two types of diagnostic tests available for Covid-19: viral tests and antibody tests. In South Africa, only the viral test is utilised for diagnosing infection.

\section{A viral test tells you if you have a current infection}

The timing of testing is critically important. If one tests too early after exposure the test can be negative. You can also test negative if you use this test after the peak stage of infection. The molecular real-time polymerase chain reaction (RT-PCR) test detects the virus's genetic material. This test is conducted by inserting a swab deep into your nose or throat to collect a specimen for testing. False-positive results can occur with PCR tests, because the coronavirus's genetic material may linger in the body long after recovery from an infection in some individuals. While we think the virus is dead 10 days post-infection, the test remains positive. In fact, in South Africa, given the shortage of test-kits, repeat testing after 10 days of a confirmed infection is discouraged. False-negative results from antigen tests may range as high as 20 to 30 percent.

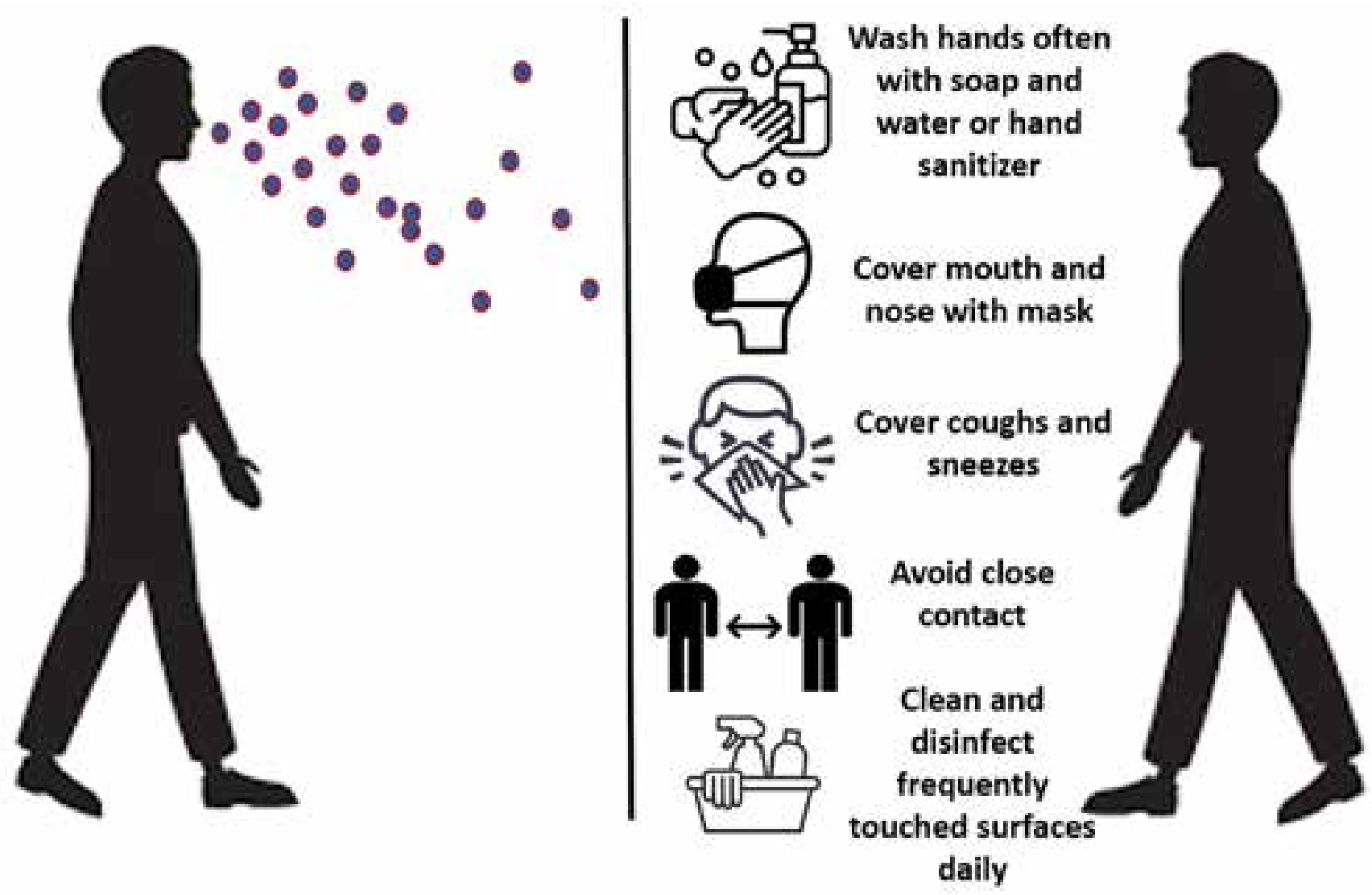

Figure 7.10: Preventive measures to avoid the spread of SARS-CoV-2. The virus spreads mainly from person-to-person between people who are in close contact with one another and through respiratory droplets produced when an infected person coughs, sneezes or talks. The best way to prevent Covid-19 is to avoid being exposed to the virus. 
An antibody test, detecting antibodies in your blood specific for proteins on the surface of the virus, tells you if you had a past infection but is not good at picking up current infection because it can take 1-3 weeks after infection for your body to make detectable antibodies. Having antibodies to the virus that causes Covid-19 might provide protection from getting infected with the virus again, but we do not know this for sure as a few cases of re-infection have recently been identified in China and the USA. We do not know how much protection antibodies provide or how long this protection might last.

Another serological test measures a person's levels of different kinds of antibodies, called immunoglobulins, all of which can be created as an immune response to an invader. They are called IgM (early), IgG (later) and IgA (formed at mucosal surfaces like the airways and intestines, but also found in the blood). These antibodies are all proteins produced by the immune system in response to an infection, but they appear at different times in the course of an infection, and they are all specific to that particular infection. They are found in the clear part of blood when it is separated from the red blood cells, which is called serum (after clotting) or plasma (without clotting).

\section{What do SARS-CoV-2 IgG and IgM levels tell us?}

Since SARS-CoV-2 is a new virus, we are still learning how our immune response works against Covid-19 and exactly how long antibodies last. Tests of total IgG and IgM levels cannot distinguish between early (IgM) and late (IgG) antibody responses and, as a result, don't provide a clear picture about whether an individual has potentially developed a longer-term immune response (IgG) or is currently infected (IgM). Alternatively, an IgG-specific serology test reveals if a person had coronavirus in the past and has developed antibodies that are highly specific to the virus.

While we don't yet know if IgG antibodies offer lasting SARS-CoV-2 immunity, the IgG-specific test does tell clinicians of past infection, which can provide important information regarding individual and population immunity levels.

In South Africa, antibody tests have only recently been approved and are primarily used for surveillance purposes (monitoring who has been infected at a population level) rather than for diagnostic purposes.

\section{Children and Covid-19}

To date, data on Covid-19 in children and adolescents remain scarce. Such young people can be asymptomatic or may present with fever, respiratory and/ or gastrointestinal symptoms. Recent studies have demonstrated that Covid-19 is generally a mild disease in children when compared with older patients. It is not 
yet known what the reasons for the difference in severity of disease are, but three possibilities exist:

- Children may have lower viral loads even if they get Covid-19. There may be a correlation between the severity of Covid-19 and the viral loads (or the duration of virus-shedding period).

- Children have lower expression levels of ACE2, when compared to adults, and, therefore, have fewer receptor sites for the SARS-CoV-2 to bind to.

- There may be a simultaneous presence of other viruses in the mucosa of the lungs and airways, common in young children, and these viruses may compete with SARS-COV-2 virus and limit its growth.

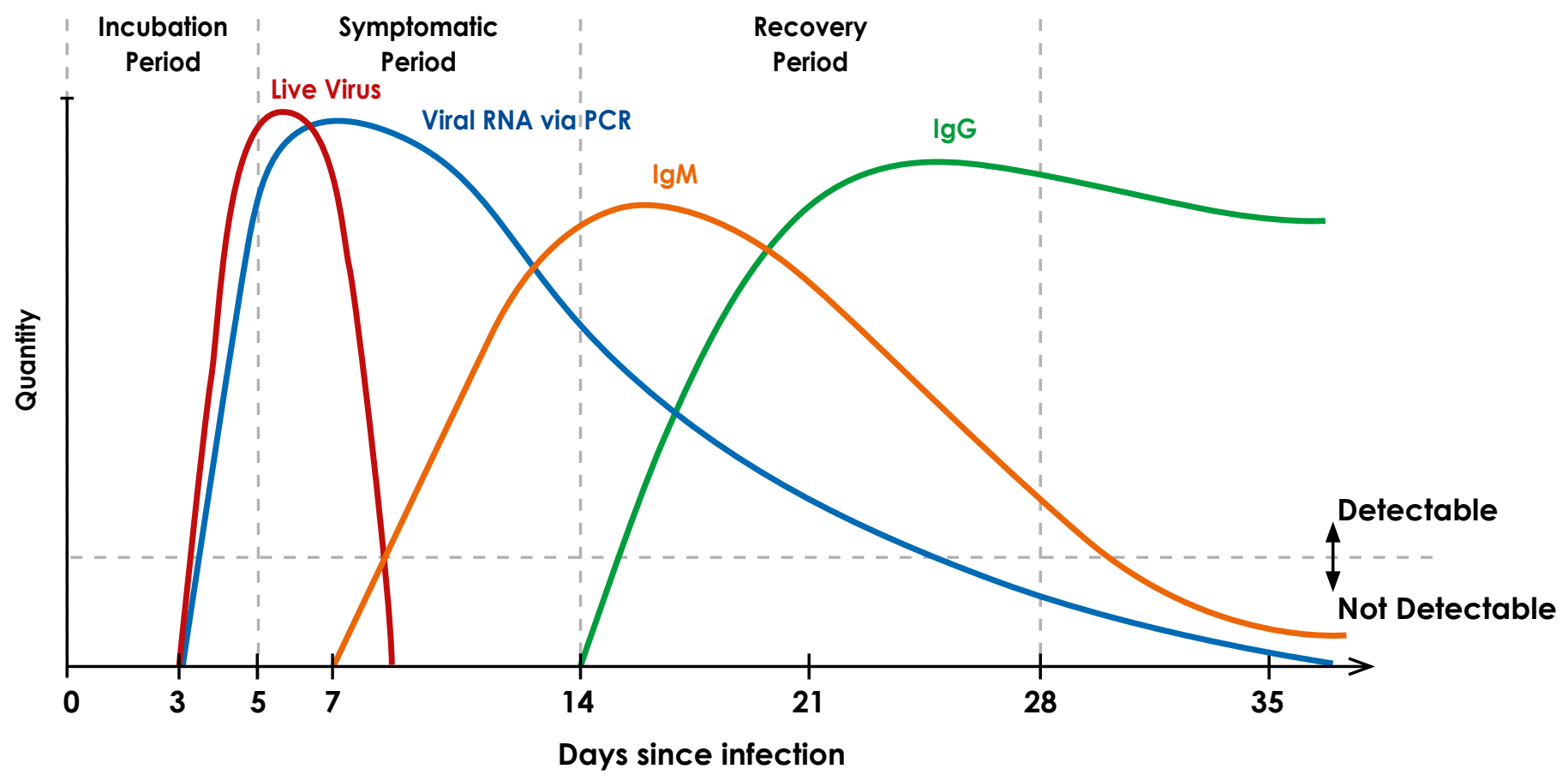

Figure 7.11: Window for detection of SARS-COV-2 infection

Reprinted with permission from: Denning et al., British Dental Journal 229 (2020) 521

There has been a record of children and adolescents with a very rare Covid19associated multi-system inflammatory condition, which seems to develop after the infection rather than during the acute stage of Covid-19. The clinical features of these paediatric cases are both similar and distinct from other well-described inflammatory syndromes in children, including Kawasaki disease, Kawasaki disease shock syndrome, and toxic shock syndrome. This Covid-19 associated multi-system inflammatory syndrome in children and adolescents is referred to interchangeably as paediatric inflammatory multi-system syndrome temporally associated with SARSCoV-2 (PIMSTS) or multi-system inflammatory syndrome in children (MISC) associated with Covid-19. 


\section{Symptoms of Multisystem Inflammatory Syndrome in Children (MIS-C)}

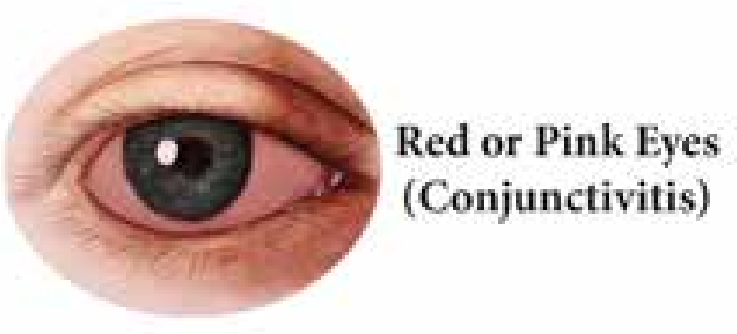

Enlarged Gland

(lymph node on one side of neck)

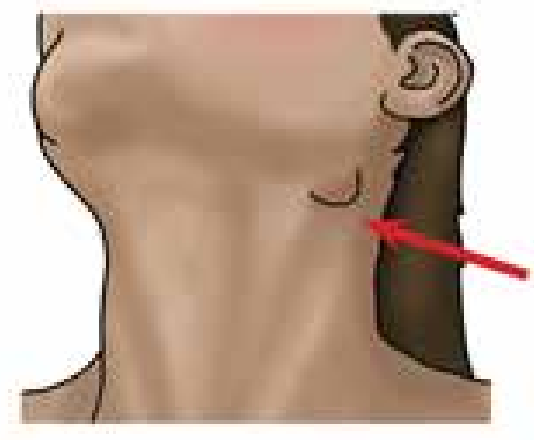

Hive-like Skin Rash

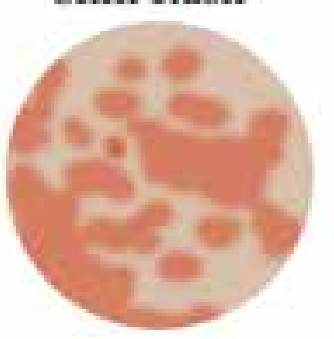

Irritability or Sluggishness

DSheri Amsel wwwexploringnature.org
Loss of Appetite

Fever Lasting

Several Days (100.4F or more)

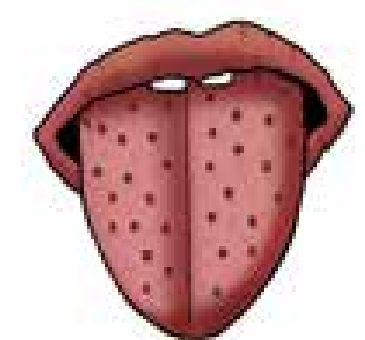

Red, Cracked Lips or Red Tongue (looks like a strawberry)
Diarrhea and/or

Vomiting

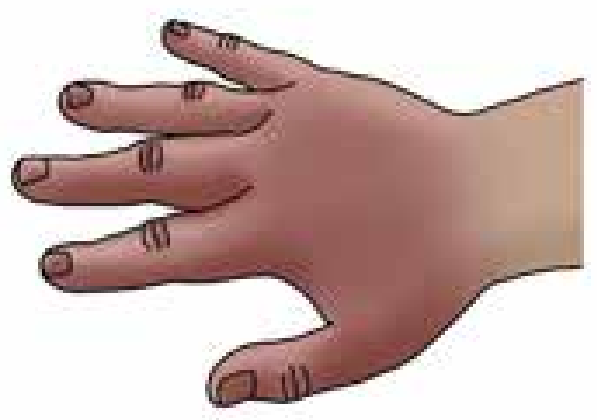

Swollen Hands and Feet

(may also be red)

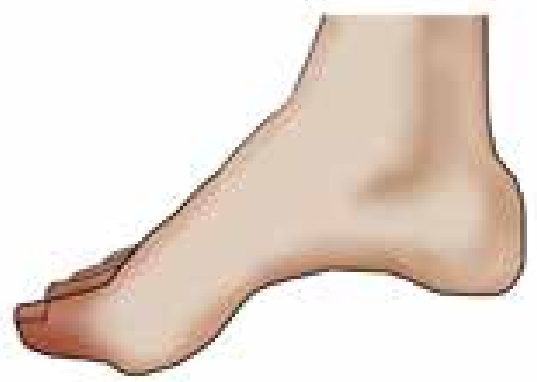




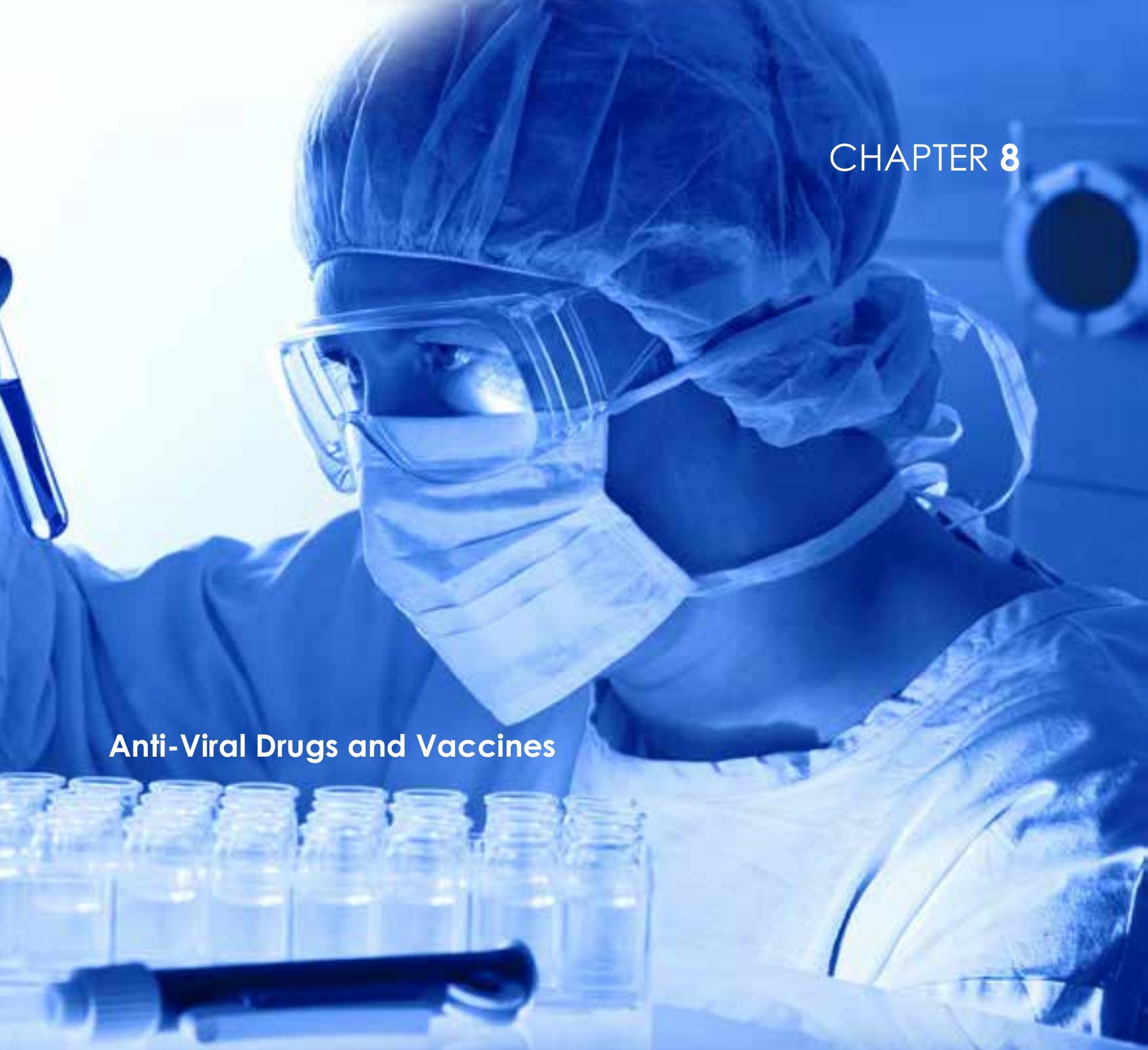

In this pandemic we have the advantage of the lessons from former pandemics and modern medicine. However, SARS-CoV-2 is a new virus only distantly related to the original SARS-CoV family. This put the whole world on a back foot, and the medical world soon informed us that the only solution for prevention of infection (besides behavioural changes) would be the development of a vaccine. It was estimated that vaccine development would take time. Some countries hoped that a rapid and widespread infection would provide populations with herd immunity but that was risky. The alternative, while a vaccine was being sought, was to try different combinations of known drugs for those who became very ill. Drugs that interfered with viral RNA replication were of choice, amongst others. Happily, a number of effective vaccines have been developed, and the challenge has become to deploy them wider. 


\section{How can drugs stop the spread of viruses?}

The development of antiviral chemotherapies has been one of the success stories of the last forty or so years, with a huge expansion in the availability of effective drugs that can limit virus replication and even cure disease.

If one understands in detail the various and often very diverse mechanisms viruses employ to enter our cells, to express and replicate their genomes once within, and to disperse themselves again, it is possible to develop drugs targeted at one or more of these processes. Possibilities include: (1) to interfere with entry of viruses into cells; (2) to prevent release of virus components inside the cell; (3) to target specific viralencoded enzymes essential to expression and processing of viral proteins; (4) to block the machinery the virus uses to make copies of their own genomes. All this can be attempted, hopefully without adversely affecting the essential work of the host cell.

\section{Why can't one use antibiotics against viruses?}

Antibiotics target a number of bacterial- or fungal-specific functions, which occur in the cells of these organisms, removed from the essential functions of the host organisms or their cells. One can specifically interfere in bacterial cell wall biosynthesis (as the antibiotic penicillin does) without affecting the host organism. One can use ciprofloxacin, which specifically targets the DNA gyrase in bacteria to stop DNA replication, but also does not affect the animal host.

The main problem here is that viruses take over the cells they infect in a way bacteria do not. Viruses use the host cell's own machinery to make the components for replicating themselves, from regulatory proteins to structural components to their own nucleic

Viruses use the host cell's own machinery to make the components for replicating themselves, from regulatory proteins to structural components to their own nucleic acids acids, whereas bacteria have their own protein-synthesising machinery and DNA and RNA-processing enzymes. Interfering with these virus processes, therefore, could mean adversely affecting the processes of the host cell. It is, consequently, much harder to precisely target virus-specific processes compared to bacterial processes because the virus often uses host processes. This means that nearly all antibiotics are useless against viruses.

Sadly, all too often, doctors prescribe antibiotics for illnesses that are actually caused by viruses. The best example of this is doctors that prescribe antibiotics for the common cold, an infection caused exclusively by viruses. Unfortunately, huge collateral damage is done by giving a person an antibiotic who does not need one. The truth is that we humans live in an exquisitely important symbiotic relationship with 

bacteria. In fact, there are over 10 times more bacterial cells in and on our body than there are human cells. In our gut alone there are 100,000,000,000,000 (1014 bacteria)! These bacteria play critical roles in keeping us healthy, including protecting the gut surface from pathogens (think of bouncers stopping people getting into a club), helping us digest food and vitamins, and critically playing an important role in our immune system. Every time we give someone an antibiotic, large numbers of these beneficial bacteria in the gut and elsewhere are killed, leaving us vulnerable and damaging our health. Use of antibiotics in infancy has been associated with later obesity, and changes in the bacterial composition of the gut due to antibiotics has been associated with many diseases from inflammatory bowel diseases like Crohn's disease to neuropsychiatric illnesses. Furthermore, indiscriminate use of antibiotics increases bacteria that are resistant to those drugs, damaging our ability to treat people with bacterial infections. Antibiotic resistance presents a global public health crisis that threatens modern medicine as we know it.

\section{What are the ways to interfere with viral replication?}

Viruses have the most varied genomes, or nucleic acid components, of all organisms. Moreover, while all cellular organisms (bacteria, archaea, fungi, plants and animals) have only double-stranded DNA as their genetic material, and all replicate their DNA in similar ways, viruses have seven different types of genetic material, based on both structure and how they replicate their genomes. Virus particles may have double- or single-stranded DNA replicating via DNA intermediate, or doublestranded RNA replicating via single-stranded RNA, or single-stranded RNA of positive or negative sense that replicates via double-stranded RNA, or single-stranded RNA or double-stranded DNA that replicates via reverse transcription from RNA to DNA and back again, or the converse mechanism. All of these, save some of the first, have virus-specific enzymes that mediate the process, meaning that it is possible, with knowledge of the structures of the proteins, to develop drugs that can specifically

Viruses have the most varied genomes, or nucleic acid components, of all organisms. jam up different types of proteins. For example, a drug known as acyclovir specifically inhibits a human herpesvirus enzyme called thymidine kinase that is vital for virus replication. The drug cidofovir, a nucleoside analogue that interferes in DNA replication, is also a potent agent for treatment of poxvirus infections, probably because the viruses do not use cell nuclear machinery for replication of their DNA.

Other drugs target the replication machinery of RNA viruses, a function that is not present in host cells: thus, chronic infections of hepatitis $\mathrm{C}$ may be effectively treated using a variety of drugs that target components of the viral replication complex, or stop RNA chain elongation. In fact, it is now possible to cure chronic hepatitis C 
infections, something the Egyptian government embarked upon as a national strategy recently.

A drug called remdesivir was developed as a broad-spectrum antiviral drug that interferes with RNA virus replication and was used with some success to treat Ebola virus infections. Remdesivir was recently re-purposed to treat COVID-19 patients and was used mainly in serious cases, for which it seemed to shorten the period of illness. A later, bigger study did not confirm this however, showing how slowly we acquire reliable knowledge in science.

\section{Why is fighting a virus like shooting at a moving target? Viral evolution and natural selection.}

Apart from viruses with big DNA genomes-poxviruses, adenoviruses, herpesviruses, iridoviruses-most virus genomes are far more susceptible to mutagenic change than the host cell DNA. This is especially true for most RNA viruses, and is due to the fact that RNA viruses generally do not have a 'proof reading' capability in their replication machinery, meaning that they mutate at a much faster rate than cellular DNA does. Consequently, natural selection operates far faster among the virus than their host cells. Influenza viruses, for example, mutate fast enough that they can 'drift' away fast enough from being recognised by host immune systems. Strains circulating a couple of years down the road may be different enough to re-infect you despite previous exposure. HIV and other retroviruses are even worse. The rate at which their genomes accumulate mutations is such that every single one of the billion or so HIV genomes made in the course of a single day in a single infected individual will have at least one difference to every other one. Such rapid evolution can lead to 'escape phenotypes', where viruses can be isolated that are resistant to previously successful chemotherapies. This occurs distressingly often with the HIV virus.

This sort of variation has its limits. Although mutations may occur at a high rate, this depends to some extent on the size of the viral genome-bigger viruses code for more functions and can tolerate fewer mutations that may impair their viability, so their nucleic acid repair mechanisms are more effective. Virus evolution does not, therefore, necessarily proceed at the same rate in the case of different viruses. For example, while HIV-1 and HIV-2 are as different from one another in terms of genetic sequence as any two randomly-picked animal descendants of the Cambrian Explosion over 500 million years ago, they are far more similar to one another in terms of structure, morphology and gene order than crayfish and humans, for example. Viruses like measles and mumps - both RNA viruses - also only have one 'serotype', despite infecting and being selected for variation in humans for centuries. This is because their surface proteins are under a strong constraint not to mutate, so as to preserve functions essential for infection of host cells. 


\section{HIV drugs: A case study}

The explosion of research sparked by the discovery and characterisation of HIV-1 and HIV-2 in the 1980s led to an unparalleled expansion of our understanding of host immunology, virus-coded structures, and how to develop drugs that targeted different stages in the virus lifecycle. This was in fact probably the spur that led to the successful development of drugs to target hepatitis $C$ and $B$ viruses, because so much expert attention was being focused on retroviruses, and how to interfere with their replication and spread.

If one divides the virus lifecycle into entrance, entertainment, and exit - a useful tool for remembering the essential stages in the process - then it is possible to differentiate entry inhibitors, replication and expression inhibitors/antagonists, and inhibitors of viral particle dissemination. HIV-1 and -2 are retroviruses, which are viruses that replicate via conversion of RNA to DNA incorporated into the host chromosomes, and back again. Retroviruses have replication and expression machinery (reverse transcriptase, integrase) that are specific to retroviruses and dissimilar to machinery of the host cell. Retroviruses also have membranes around their particles derived from host cells, but containing additional envelope proteins whose function is to fuse the virus particle with cell membranes in order to deliver the virus genome and replication machinery into the cell. Regulation of the expression of the viral DNA phase relies on virus-encoded proteins, and the virus proteins need to be processed by a virus-encoded protease for proper assembly of particles within the cell and for those particles to reach their final form to be infectious. All of these stages can be targeted with drugs that exploit knowledge of the molecular mechanisms that

Influenza viruses, for example, mutate fast enough that they can "drift" away fast enough from being recognised by host immune systems. make them work. Developing such drugs took a long time and a great deal of research. But it is now safe to say we understand the life cycle and structures of HIV-1, and its expression and replication machinery better than just about any other organism on this planet.

A wide variety of drugs are given to people with HIV infections to keep their viral loads low, or even as pre-exposure prophylactics. Drugs that block virus particles from attaching to cells are known as attachment inhibitors. These include very expensive monoclonal antibodies (mAbs), which block CD4 receptors on cells, compounds such as fostemsavir (FTR, FDA licenced in 2020), which binds to the viral gp120 and blocks its binding to cell-surface CD4 protein as the first stage of entry, and other drugs that bind to and block viral attachment to the second of two cell proteins vital for viral entry. Post-attachment inhibitors, or fusion inhibitors, work at the next stage of infection and block the virus from entering the cell after binding to the CD4 and CCR5 proteins by fusing membranes. 
Drugs that specifically interfere with the replication machinery of the virus are nucleoside analogues and non-nucleoside reverse transcriptase inhibitors (NRTIs and NNRTIs, respectively). Other drugs interfere with the virus protease that is vital for proper processing of virus proteins. Integrase inhibitors block the action of the viral integrase enzyme, which is necessary for the HIV DNA form to integrate into host chromosomes.

South Africa presently has nearly 8 million people living with HIV-1 and, with over 4 million on treatment, has about $20 \%$ of the world's total number of people who are on HIV therapeutics. The initial or first-line therapy regimens for previously untreated patients in 2017 were chosen from among the following combinations:

- A mixture of the NRTIs TDF and FTC or 3TC, plus the NNTRI EFV

- NRTIS TDF + FTC/3TC plus integrase inhibitor DTG

- TDF + FTC/3TC plus NNTRI RPV (if viral load > 100000 copies $/ \mathrm{mL}$ )

- Second-line regimens-given when first-line treatment is not working-preferably include two NRTIs and a RTV-boosted protease inhibitor: this involves using low-dose RTV, which inhibits the breakdown of the added inhibitor.

In 2019, South Africa introduced an advanced therapy regime consisting of three drugs in a single pill known as TLD, to be taken daily. This contains the integrase inhibitor Dolutegravir (DTG), as well as NRTIs 3TC and TDF. DTG is the drug of choice for people living with HIV in high-income countries as it has fewer side effects and fewer negative interactions with tuberculosis medicines, which is a bonus given the very high rate of co-infection of people in South Africa with HIV and TB.

\section{What has been the success of existing anti-viral drugs against Covid-19?}

Given the burgeoning in recent years of the antiviral drug field, it was natural for researchers to turn to tried and proven agents successful against other viruses to attempt to treat Covid-19, the disease caused by the newly-emerging betacoronavirus SARS-CoV-2. This is a single-stranded RNA virus that replicates similarly to hepatitis $C$ virus ( $H C V$ ). However, the viruses are sufficiently different from one another that therapies targeting $\mathrm{HCV}$ have no guarantee of being effective against SARS-COV-2.

Early, small observational studies of chloroquine and hydroxychloroquine (CQ and $H C Q$ looked promising. Both are cheap drugs with a well proven safety record in humans. They are known to act to prevent the acidification of internal vesicles in cells of the type that SARS-CoV-2 can fuse with and, thus, allowing its genome to enter. However, while both these drugs worked in cell culture experiments against both SARS-COV-2 and the related MERS-CoV, early studies in Covid-19 patients, 
which were often coupled with the macrolide antibiotic azithromycin for its antiinflammatory actions, remained inconclusive. The same was true for the antiretroviral combination lopinavir/ritonavir. More recently, however, the UK's adaptive multiarmed randomised controlled trial, RECOVERY, proved that neither HCQ nor lopinavir/ ritonavir had any effect in reducing mortality in Covid-19. They are no longer used. The WHO's multi-country SOLIDARITY trial also failed to show benefit.

However, a breakthrough in treatment of Covid-19 came from the RECOVERY trial, which demonstrated that dexamethasone reduced mortality in patients admitted to hospital requiring supplemental oxygen. Patients requiring ventilation had a $33 \%$ reduction in death, with those requiring lesser oxygen support having a reduced mortality of $20 \%$. No benefit was observed in patients admitted to hospital but not requiring supplemental oxygen. Dexamethasone or its equivalent, e.g., prednisone, is now standard of care for severe, hospitalised Covid-19 patients worldwide.

Hopes for remdesivir, a re-purposed antiviral nucleoside analogue first used in Ebola patients, were also high following a US study that showed that it significantly reduced recovery time if people were treated early ( 11 days vs. 15 for untreated), but was less effective in severely ill patients. This was not confirmed in a much larger, later study. Furthermore, the RECOVERY trial proved that remdesivir had no effect on mortality in severe Covid-19.

There have also been reports of success in reducing the death rate among severely ill patients by use of infused convalescent plasma, which prompted the FDA in the USA to issue an "emergency use authorisation (EUA)" to allow hospitals to try it. Monoclonal antibodies are also under study. Bamlanivimab, for example, has had some success in treatment of non-hospitalised patients at risk of progressing to severe disease, and REGN-COV2, a cocktail of two monoclonal antibodies, is also in late stage trials.

IL-6 receptor inhibitors, such as tocilizumab, also gained traction in the early phase of the pandemic, with putative effect on the cytokine storm seen in severe COVID-19. Although IL-6 inhibitors reduced inflammatory responses in patients with extensive lung involvement, and who had elevated IL-6 concentrations, tocilizumab has yet to show any effect on short term mortality, but may reduce the risk of mechanical ventilation in hospitalised COVID-19 patients.

\section{How do vaccines work? Training the body's immune system}

A vaccine may be defined as a substance that produces an immune reaction, subsequently leading to an acquired immunity against a natural micro-organism. The advent of vaccines ranks among the most important developments in medical and veterinary science of the last three hundred years. The term vaccine covers a wide range of agents, including attenuated live bacteria and viruses, killed whole-cell or 
whole-virus-particle vaccines, isolated components of disease agents, recombinant proteins made in cell cultures, parts of viruses expressed in other live vaccines, and synthetic compounds such as bacterial carbohydrates.

Whatever its form, the simple requirements for a vaccine are that it be safe and that it work. Unlike therapeutics given to people having a disease, vaccines are administered to healthy people, and generally to children. Consequently, there is a stringent requirement to prove their safety beyond the requirement that they prevent disease.

Vaccines work by eliciting adaptive immune responses in their recipients that will protect them against disease caused by the selected agent. They do this by exposing the immune systems of the vaccines either to parts of the actual pathogen-in the form of subunits, or synthetic or killed vaccines-or to a whole organism that does not cause disease. For example, the original smallpox vaccine-later called vaccinia virus-was derived either from a horse-pox virus that infected a cow, or from an actual cowpox virus. This was an agent similar enough to the actual smallpox virus to elicit the right immune response, but it did not cause severe disease. Its use allowed the complete eradication of the human disease in the late 1970s. This was the first human disease ever to have been eradicated. This feat was followed in 2011 by eradication of the rinderpest virus of cattle and wild animals

Influenza viruses, for example, mutate fast enough that they can "drift" away fast enough from being recognised by host immune systems. as the result of a live vaccine campaign over many years. The modern measles, mumps, and rubella vaccine consists of viruses that were derived from the original virulent viruses via extensive culture and selection, and which now do not cause disease. There are both live and killed polio virus vaccines, which are derived by extensive tissue culture selection, in the first case, and by chemically inactivating live, virulent polio viruses. in the second case.

Subunit vaccines are not a modern idea. The diphtheria and tetanus vaccines are both inactivated toxins derived from cultured live bacteria. Moreover, recombinant virus protein vaccines produced via genetic engineering by bacteria, yeasts or insect cells are now well established in human medicine. These include hepatitis $B$ virus-like particles (VLPS) produced in yeast cells, and human papillomavirus VLPS produced in yeast or insect cells.

\section{How have vaccines helped to reduce infant mortality?}

It is reliably estimated that over 3 million children's lives are saved every year worldwide by use of vaccines that are included in the Expanded Programme on Immunisation (EPI), established by the World Health Organisation (WHO) in 1977. 
The first diseases targeted were diphtheria, whooping cough, tetanus, measles, poliomyelitis, and tuberculosis. This list has since been expanded quite considerably, although implementation is subject to individual country policies and abilities.

South Africa presently targets three diseases for eradication: poliomyelitis, measles, and neonatal tetanus. A further seven diseases targeted by the programme are diphtheria, pertussis, tuberculosis, hepatitis B, Haemophilus influenzae type B (Hib), rotavirus, and pneumococcal infections. Poliomyelitis has effectively been eradicated in South Africa, and wild-type polio has been eradicated in Africa as of 2020 .

\section{How safe are vaccines?}

Any vaccine that has received regulatory body approval has passed extremely stringent conditions for its release and has been proven to be safe and efficacious in large, and usually international, Phase III clinical trials and subsequent observational studies. Although this does not completely eliminate the possibility of rare adverse events, vaccines that are used in the EPI programmes worldwide are among the safest medicines ever made, and the benefits have been shown to greatly outweigh the small risk of rare adverse events.

\section{What has been the process leading to the emergence of several effective vaccines against Covid-19?}

During the early stages of the pandemic, many expressed scepticism that an effective vaccine would become available any time soon, pointing out that the time period from initial vaccine development through a series of trials and, finally, to approval often takes over a decade. Yet despite

the many obstacles, we see that several highly effective and safe vaccines have emerged. A major reason for this is that pharmaceutical companies, in many cases with substantial financial support from governments, has compressed the timeline by overlapping many of the phases of this process. For example, the infrastructure for producing vaccine in massive quantities was put in place early on, before the success of clinical trials, and actual production in mass quantities of vaccine preceded 
approval and in some cases the final results of clinical trials. From a financial point of view, such a compressed timeline entails considerable extra risk, in case the vaccine fails. But many governments were willing to subsidise these risks given the considerable losses that result from a continuation of the pandemic.

The accelerated development for Covid-19 vaccines was in part made possible by the fact that many of the developers had already been working either with related coronavirus vaccine candidates, for the Middle Eastern respiratory syndrome coronavirus (MERS-COV) or the original severe acute respiratory syndrome virus (SARS-COV)-and could simply swap in SARS-CoV-2 genes or proteins, or had welldeveloped vaccine 'backbones' like measles and VSV, making it easy to adapt them for Covid-19. These factors, and the willingness to collapse the clinical testing regime, so as to allow concurrent Phase $1 / 2$ and $2 / 3$ testing, means that development time could be reduced to as little as ten months to a year.

Of the over 150 vaccines development efforts to date, ten vaccines have already succeeded and been approved under "emergency use authorisation" in at least one country, and this number can be expected to grow.

\section{Covid-19 vaccines fall into the follow general categories:}

- Genetic vaccines, consisting only of RNA or DNA encoding the spike protein, or the main virus particle outer protein;

- 'Killed' or inactivated whole virus particle vaccines;

- Subunit, or nanoparticle, vaccines, which consist only of the spike protein made in a recombinant expression system;

- Live virus vaccines, such as several different kinds of adenoviruses or measles vaccine or vesicular stomatitis virus (VSV, also used for Ebola) that carry the gene for the spike protein

\section{Attenuated vaccines, with the live SARS-CoV-2 virus itself.}

The Pfizer-BioNTech and Moderna vaccines are of the category of the genetic vaccines, using messenger RNA encoding the spike protein. The Oxford AstraZeneca, Johnson and Johnson, Sputnik $V$ and Chinese Ad5-nCoV vaccines use an adenovirus vector to deliver the spike protein in order to provoke an immune response. The Chinese BBIBP-CorV and CoronaVac vaccines, as well as the Russian Covivac vaccine and Indian Covaxin vaccine, on the other hand, are inactivated virus vaccines. The EpiVacCorona and RBD-Dimer vaccines are subunit vaccines. 
Once a Covid-19 vaccine has been proven safe and effective, what challenges will remain in distributing it widely and making sure that a sufficient number of people are vaccinated so that life can return to the previous normal?

Although developing, testing, and licensing a vaccine involves many formidable challenges, once these challenges have been overcome the story is not over. We now have several vaccines that have been proven both safe and efficacious, but large-scale manufacture and distribution poses new, equally formidable challenges. Manufacture is a major challenge for certain types of vaccines. Those merely requiring a live virus to be grown, such as the adenovirusbased candidates, are probably the easiest to produce, as the volume of material to be produced would be much less than for subunit vaccines, which typically require much more material to be made and purified, as they are not infectious. Killed vaccines are one of the quickest ways to make the product, but

As this booklet goes to print (July 2021), a small fraction of the world's population has been vaccinated, even in the affluent, developed countries.

this requires that a very large amount of live, virulent SARS-CoV-2 be grown. This is problematic due to the stringent safety requirements imposed when working with live, unmodified virus. The production volumes required for the non-live vaccines are such that the world may simply not have the capacity to make the required amounts in less than a couple of years.

Distribution is also potentially a problem, as certain of the vaccines require refrigeration to as low as $-80^{\circ} \mathrm{C}$ for storage and transport, which may pose a serious obstacle for rolling these out to remote locations in underdeveloped countries.

As this booklet goes to print (July 2021), a small fraction of the world's population has been vaccinated. While many in affluent countries have been vaccinated, only a small fraction of those in developing countries have been so fortunate. The fraction of people having been fully vaccinated in the US, Europe, and the UK are $56 \%, 48 \%$, and $49 \%$, respectively. The comparable fraction for South Africa is $4.8 \%$.

It remains to be seen how manufacturing capacity is scaled up and the geopolitical issues of vaccine distribution are handled. 
As we write there are several vaccines in circulation around the world. The well-known ones are Pfizer-BioNTech, Moderna, Johnson and Johnson, Oxford Astrazeneca and NovaVax, and a few others. Their use depends on their availability in different countries. There is a rush in many of the rich countries to vaccinate a huge number of people to reach herd immunity, a point of safety in which the vaccination of a certain percentage of people will slow or stop the virus from spreading to those who are not vaccinated.

Poorer countries like South Africa have been slow in their vaccination roll-out. We initially procured one million Oxford Astrazeneca vaccines and did not use them because it showed low efficacy against the Beta variant (B.1.351 or 501Y.V2) in South Africa. The Johnson and Johnson vaccine is currently the preferred choice and is now being used to vaccinate our healthcare workers. Anti-Covid-19 is a developing story worldwide.

While the vaccines presently being used worldwide do not protect vaccinees completely against infection, they do appear to protect vaccinated people against severe disease and hospitalisation, whichever variant (Alpha, Beta, Delta or the new Gamma) they have been infected with.

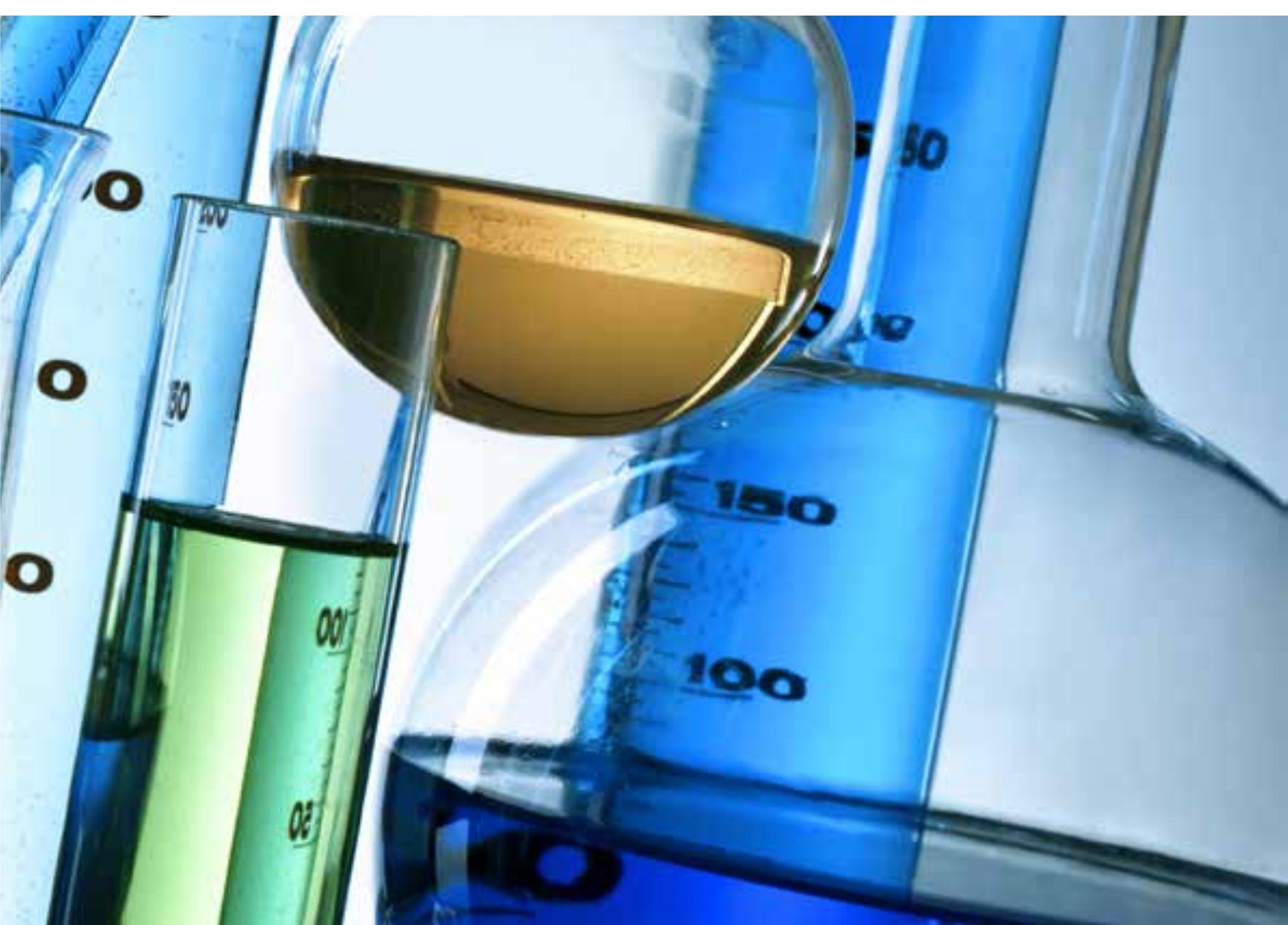




\section{CHAPTER 9}

The South African Healthcare System and Covid-19

South Africa has a dual healthcare system. For the majority of South Africans, healthcare is provided for by the state, through the national, provincial and local healthcare systems. Healthcare is provided attertiary (academic teaching hospitals, for example Groote Schuur, Tygerberg, King Edward VIII and Baragwanath teaching hospitals), secondary and regional hospitals and local clinics. A parallel private healthcare system is based on an insurance scheme and is funded by medical schemes (such as the Discovery Health). Private healthcare is available to more affluent South Africans able to pay the premiums for these schemes. All systems are regulated by the Health Ministry. The state system is overstretched and struggles to provide adequate healthcare for the majority of South Africans unable to afford medical insurance. The coronavirus pandemic has aggravated this situation. A further development during the pandemic is the public-private healthcare mix in which beds were made available between sectors when the situation

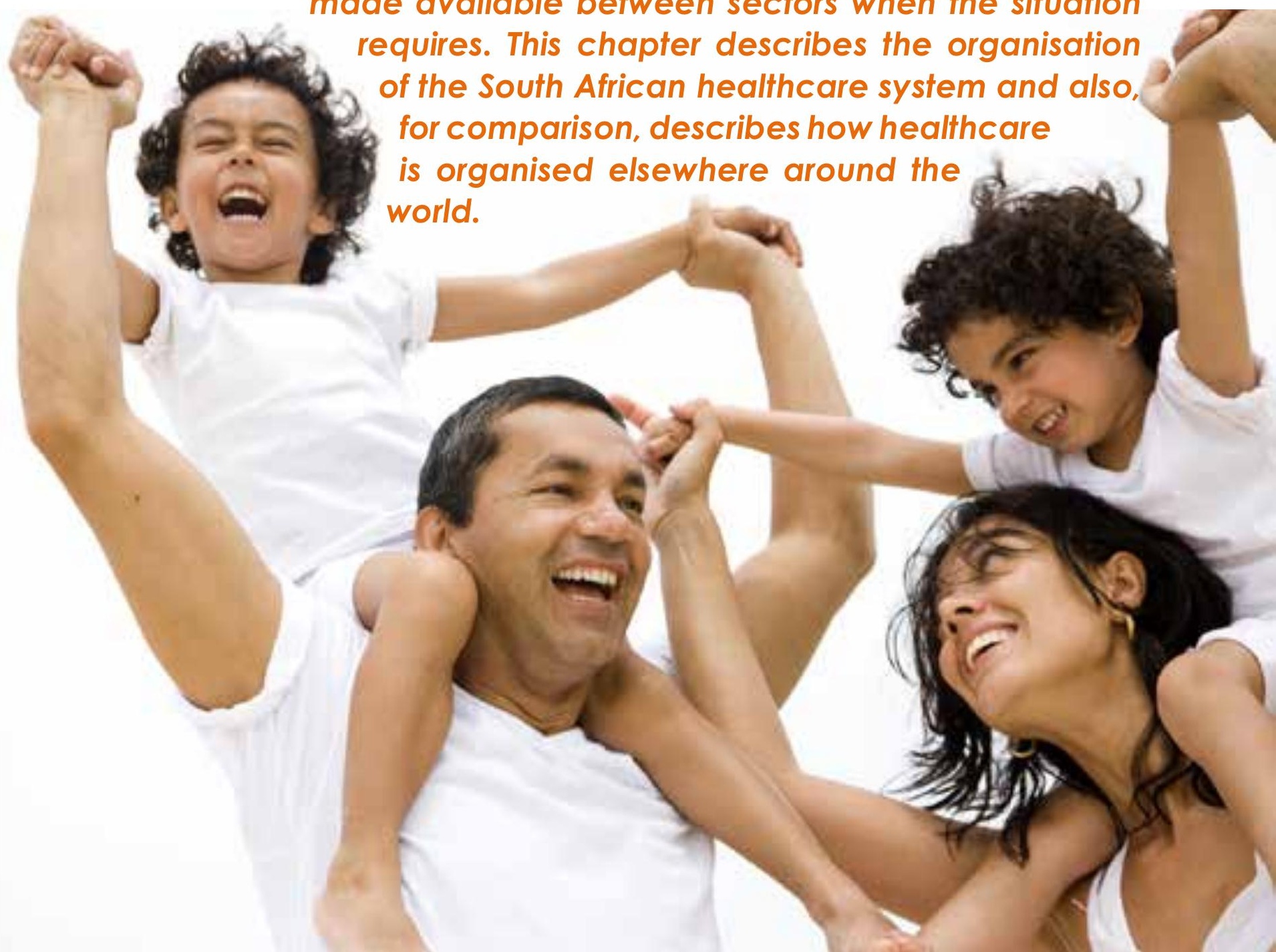


This chapter provides an overview of the South African healthcare system explaining how key features of this system have both supported or detracted from an effective response to the Covid-19 pandemic. The South African health system may be divided broadly into four parts, three of which are governmental and one of which is private. The national, provincial, and local governments work together to deliver health service. The national government defines a national policy and oversees its implementation. The provincial government has the constitutional mandate for health services held concurrently with the national sphere. Finally, local governments have a mandate for health functions not involving clinical healthcare services, including environmental health, sanitation, and aspects of population health.

The fourth part of the health system consists of private healthcare providers and is funded by private health insurance (referred to as 'medical schemes'). This private sector is, however, also governmentally regulated, mainly through national legislation falling under the jurisdiction of the Minister of Health. All health professionals, medicines, medical products, medical devices, pharmacies, and medical schemes are regulated. A regulator, the Council for Medical Schemes, which reports to the Minister of Health, supervises medical schemes. Private hospital licensing, however, occurs at a provincial level (Figure 9.1).

The emergence of provincial governments and medical schemes as the predominant vehicles for the delivery of health services in South Africa is an outcome of the incremental interplay between the changing needs of the population and policy over a period of 200 years. The present institutional context is, consequently, quite entrenched.

\section{Financing the health system}

The public health system can be divided into five elements: general government (national and provincial); local authorities; the health system of the national defence force; social insurance arrangements (compensation for occupational injuries and diseases, compensation for occupational diseases affecting mineworkers, and the Road Accident Fund); and subsidies to medical scheme members. The focus of this section is on the main elements: the financing of publicly delivered services through national, provincial and local government structures; and the private health system funded by medical schemes.

The Constitution broadly establishes a multi-level general government system, with significant autonomy allocated to the three spheres of Government. While the system is subject to continuous legislative development, which influences the distribution of functions and the relative degrees of autonomy, broadly speaking the financial architecture of the South African public system corresponds to what is termed 'fiscal federalism'. 
The bulk of government revenue is raised through national taxes and allocated to national, provincial and local government through the Division of Revenue Act (DORA). The allocations to national departments by the National Legislature take the form of voted budget allocations. The voted allocations to both provincial and local governments, however, become revenue for these structures. Their legislative structures subsequently vote on final allocations, taking account of both their own revenue sources and that derived from national government. (See Figure 9.2 for an illustration of the flows).

Transfers from national government take two basic forms: a formula-based unallocated block grant, which provides general revenue to both provincial and local authorities; and conditional allocations, which, where required, seek to achieve convergence between national, provincial and local governments. These allocations and the relevant conditions are specified in the DORA each year. Provincial governments, in particular the health function, provide subsidies to local authorities to render services falling within the functional responsibilities of the province.

In this system, each sphere of government is able to receive funds as revenue from their own taxes, user fees and inter-governmental transfers. While revenue transfers can technically go in any direction, to date they tend to flow from the higher sphere to lower spheres.

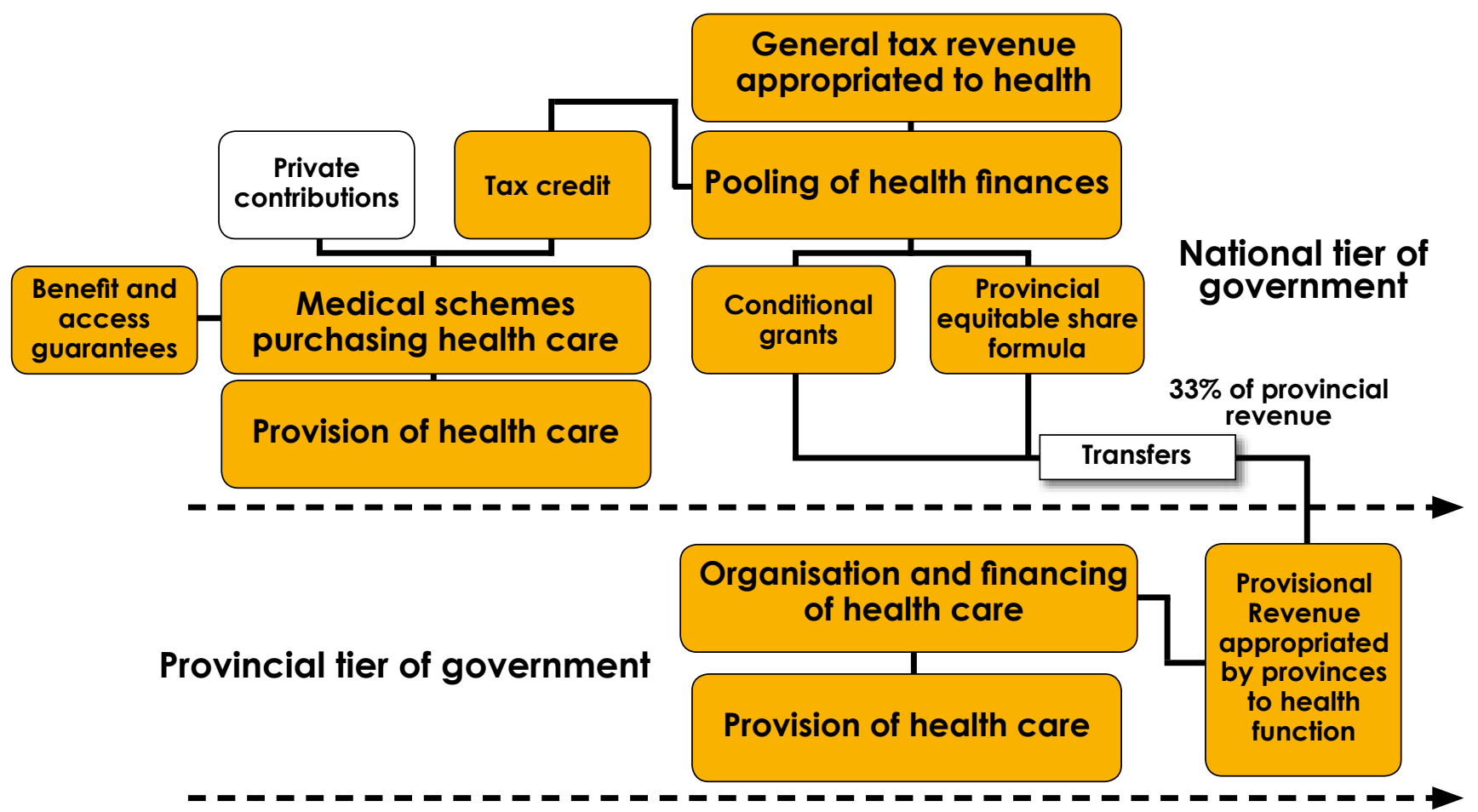

Figure 9.1: High-level overview of South Africa's present universal health coverage framework. Credit: Alex van den Heever 
The distribution of national government expenditure is heavily weighted to national government and the provinces, which was at $47.7 \%$ and $43.3 \%$, respectively, in 2018. Local government only received around $9 \%$ in 2018, with most of their budgets financed through their own taxes, utility fees and transfers from provincial governments.

For provinces, the formula-allocated unconditional provincial equitable share grant (PES) accounts for the bulk of their revenue, which was $82.4 \%$ of the national allocation in 2018. Conditional grants, the largest share of which are for health functions, stood at $17.6 \%$ of their nationally sourced revenue in 2018 . Overall, the national allocation to provinces came to R571 billion in 2018.

National revenue flowing to local government is made up of three transfers: the unallocated local government equitable share grant (53.0\% in 2018); conditional grants (much of which is for the health function) (36.5\% in 2018); and a distribution of the revenue raised from the fuel levy (10.5\% in 2018). In 2018 , the overall allocation to local governments from national government amounted to R1 18.5 billion.

The largest conditional grants relating to the health function for expenditure in 2018 were the Comprehensive HIV, AIDS and Tuberculosis Grant at R19.9 billion; the National Tertiary Services Grant (NTSG) at R12.4 billion; and the Health Professions

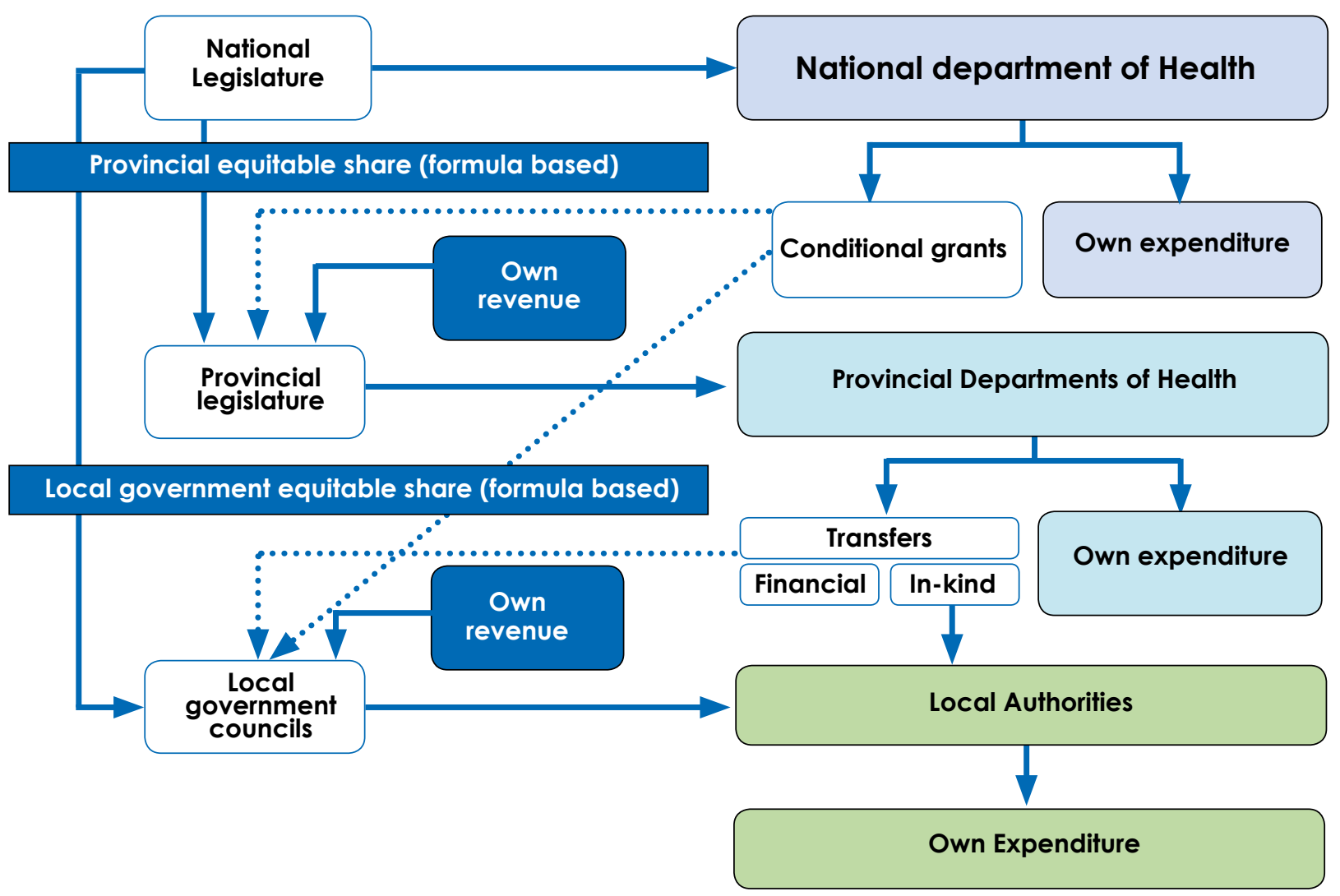

Figure 9.2: Organisation of public health financial flows-revenue and expenditure. Credit: Alex van den Heever 
Training and Development Grant (HPTDG) at R2.8 billion. Capital transfers in 2018 for the Health Facilities Revitalisation Grant involved an additional R5.8 billion. In total, health conditional grants in 2018 came to R35.3 billion.

Two conditional grants are of particular importance for the provinces of Gauteng and the Western Cape. These are: the NTSG, which theoretically funds cross-boundary flows from other provinces for the use of highly specialised services concentrated in these provinces; and the HPTDG, which theoretically funds the additional costs to the health service for the teaching and research functions carried out by academic complexes concentrated in these provinces. In the absence of these grants, both these provinces would be unable to sustain their more complex services and educational functions.

The relationship between these grants and the services they are supposed to fund has never been built into the funding model and both the provincial and national departments appear to lack the capacity to properly negotiate the values and specificity of the grants. Despite numerous reviews by the Financial and Fiscal Commission, culminating in a published report in 2006 (van den Heever, Nthite, \& Khumalo, 2006), no changes have been made to the grant designs. Due to the failure to adjust these grants, or provinces to properly negotiate their value, both the Western Cape and Gauteng are likely to face growing financial constraints in maintaining their highly specialised services over the next ten years.

\section{The public-private mix}

When expressed as a percentage of Gross Domestic Product (GDP), public and private health system expenditures have remained relatively stable over a long period. However, both the public health systems and medical schemes saw a structural increase from $2.8 \%$ and $3.2 \%$, respectively, in 2000 to $3.9 \%$ and $3.9 \%$, respectively, in 2018. Out-of-pocket expenditure, at least as reflected by the World Health Organisation, stood at an estimated 0.6\% of GDP in 2014 (World Health Organisation, 1995 to 2014), which is fairly good by international standards.

While the expenditure trends have remained stable and rising, the populations served by the two systems have not. Dramatic cost increases in the medical schemes system appear to have constrained increases to below overall population increase (a drop from $19.8 \%$ of the total population in the late 1990 s to around $16 \%$ at present), with the public sector catchment population increasing slightly faster. By 2018 the public sector needed to protect 48 million people while medical schemes offered coverage to 8.9 million people (Table 9.1).

While the amount spent on the private sector via medical schemes has remained fairly constant (as a percentage of GDP) over time, there has been a dramatic shift in hospital services away from the public sector. 
Until 1986, most people covered by medical schemes purchased their hospital services from the public sector, as higher-income groups and medical scheme members have always needed to pay for public hospital services. From 1986 to 2010 , the number of private acute hospital beds in the private sector increased from 6,125 to 31,067 , while beds in the public sector declined from 117,842 to 88,920 (Table 9.2). In the period after 2000 , the public sector explicitly de-prioritised hospitalbased services in favour of primary care-or district health services. Within the public health budget, there has also been a substantial diversion of resources to HIV and AIDS funding.

Table 9.1: Health expenditure in South Africa from 2000 to 2018

\begin{tabular}{llll|llll}
2000 & 2005 & 2010 & 2015 & 2016 & 2017 & 2018 \\
\hline
\end{tabular}

Public health compared to medical schemes expenditure (2018 prices)

\begin{tabular}{rrrr|rrrrr} 
Public health & 75913 & 102094 & 157255 & 183090 & 185529 & 191167 & 197142 \\
Medical schemes & 84471 & 119915 & 154792 & 178423 & 181462 & 189384 & 193332 \\
\hline Total & 160384 & 222009 & 312047 & 361513 & 366991 & 380551 & 390475 \\
\hline
\end{tabular}

Catchment populations for the public and medical schemes sectors

\begin{tabular}{rrrr|rrrr} 
Public health & 32119 & 36140 & 39566 & 44555 & 45648 & 46832 & 48030 \\
Medical schemes & 6454 & 6836 & 8316 & 8792 & 8865 & 8872 & 8891 \\
\hline Total & 38573 & 42976 & 47882 & 53347 & 54513 & 55704 & 56922 \\
\hline
\end{tabular}

Public health compared to medical schemes expenditure: \% of GDP

\begin{tabular}{cccc|cccc} 
Public health & $2.8 \%$ & $3.0 \%$ & $3.7 \%$ & $3.8 \%$ & $3.8 \%$ & $3.9 \%$ & $3.9 \%$ \\
Medical schemes & $3.2 \%$ & $3.5 \%$ & $3.6 \%$ & $3.7 \%$ & $3.7 \%$ & $3.9 \%$ & $3.9 \%$ \\
\hline Total & $6.0 \%$ & $6.5 \%$ & $7.4 \%$ & $7.5 \%$ & $7.5 \%$ & $7.8 \%$ & $7.8 \%$ \\
\hline
\end{tabular}

Public health compared to medical schemes expenditure: Per capita (2018 prices)

\begin{tabular}{|rrrr|rrrr|} 
Public health & 2364 & 2825 & 3975 & 4109 & 4064 & 4082 & 4105 \\
Medical schemes & 13087 & 17543 & 18614 & 20293 & 20470 & 21346 & 21744 \\
\hline National average & 4158 & 5166 & 6517 & 6777 & 6732 & 6832 & 6860
\end{tabular}

Source: Alex van den Heever 


\section{The South African hospital system}

The public sector presently has 86,579 usable beds, down from the 88,920 beds in 2010, as shown in Table 9.2. Of these, 30,265 are district hospital beds; 9,086 are central (academic) hospital beds; 5,810 are provincial tertiary hospital beds; and 24,096 are regional hospital beds (see Table 9.3 for the public hospital definitions). Overall, this implies a bed to 1,000 population ratio (public sector) of 1.8. This is not too far off from (national) country comparators such as Chile (2.1), Brazil (2.3), United Kingdom (2.6), with most countries also showing a downward trend over time. It is, however, significantly lower than the ratio of 4.4 , corresponding to the private sector medical schemes population.

Table 9.2: Private and public hospital and bed estimates (from 1976 to 2010)

\begin{tabular}{|c|cc|cc|}
\multirow{2}{*}{ YEAR } & \multicolumn{2}{|c|}{ PRIVATE } & \multicolumn{2}{c}{ PUBLIC } \\
\hline $\mathbf{1 9 7 6}$ & Hospitals & Beds & Hospitals & Beds \\
\hline 1986 & 25 & 2,346 & & 117,842 (est) \\
\hline 1989 & 65 & 6,125 (est) & & \\
\hline 1998 & 101 & 10,936 & & 107,634 \\
\hline $\mathbf{2 0 1 0}$ & 216 & 20,908 & 343 & 88,920 \\
\hline
\end{tabular}

Source: Alex van den Heever, 2012

Strategic decisions regarding the direction of public hospital beds are made by provinces and are subject to their specific constraints without regard to an explicit national policy framework. Although it would be possible for national government, via the National Department of Health, to use conditional grant allocations to achieve such an alignment, options along these lines have not been exercised to date, despite recommendations to do so by the Financial and Fiscal Commission (van den Heever et al., 2006). 


\section{Hospital type}

Central hospitals (academic)

\section{Description}

Hospitals with teaching responsibilities containing a high concentration of subspecialist services. These hospitals serve populations beyond the province in which they are located.

Hospitals with little or no teaching responsibilities that contain subspecialist services, but which only serve the province in which they are located and can draw patients from the entire province.

Hospitals with a strong focus on general specialisations such as obstetrics and gynaecology, paediatrics, and orthopaedics and only serve the provinces in which they are located.

Psychiatric hospitals (acute)

Acute psychiatric hospitals offer specialised psychiatric care beyond the provinces in which they are located.

Include mainly rehabilitation and infectious disease hospitals.

Due to the high incidence of TB in South Africa, the public system offers an extensive system of TB

Tuberculosis (TB) hospitals hospitals. While the principal focus is on ordinary TB cases, they must increasingly cater for various forms of drug resistant TB.

Specialised chronic hospitals
Long-term chronic psychiatric care has typically been offered in either public facilities or contracted private facilities. 
Although some private hospitals are specialised (e.g. maternity, psychiatric, day, etc.), many are general hospitals with a mix of specialisations available. Data on private hospitals is, however, not routinely published by either the public or private sector, with information often available only from unverifiable proprietary data sources. However, data is available on request from various hospital groups and associations. Based on these sources, a dataset has been compiled by the author. The estimates for 2016 (and broadly consistent with 2020) by bed type are provided in Figure 9.4.

\section{Hospital systems response to the COVID-19 pandemic}

An important concern with respect to the COVID-19 outbreak in South Africa was whether the hospital system as a whole could accommodate the peak demand for critical care beds. By the time the first cases were detected in South Africa in March 2020 , it had become clear that a consistent proportion of infected individuals become so severely ill that they require access to critical care services. In particular, a need arises for intensive nurse supervision, oxygen, and, in case of further deterioration, ventilation. Ventilation is typically only available to intensive care unit (ICU) beds. The ICU bed count, therefore, serves as an approximate proxy for the availability of ventilators.

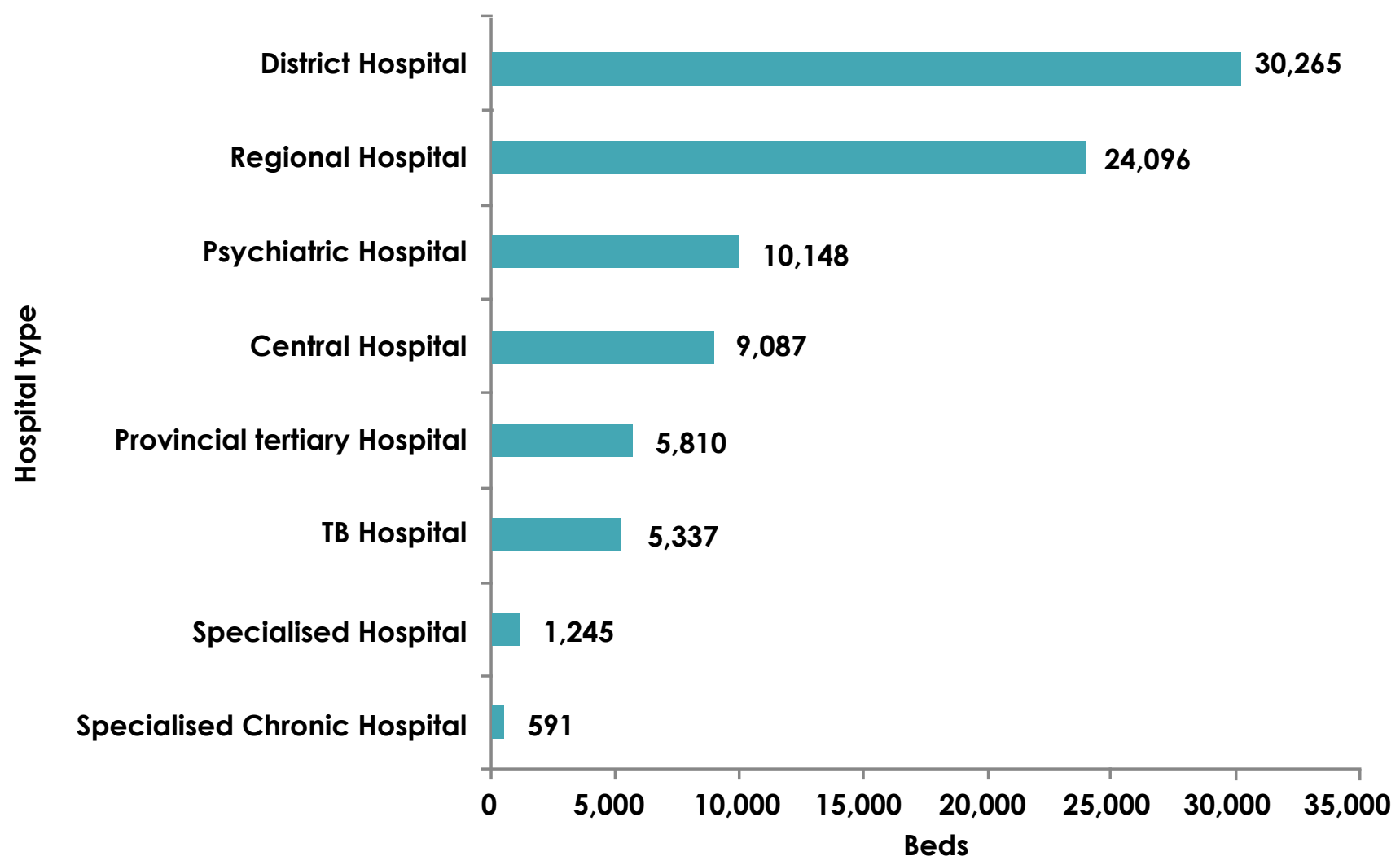

Figure 9.3: Public hospital beds by type of facility-usable beds in 2013 
While South Africa's overall bed levels appeared sufficient to cope with less severe admissions, it was initially unclear whether sufficient critical care beds, and especially ventilators, would be available to cope with peak demand.

Without accounting for occupancy for non-Covid-19 cases, at the beginning of the outbreak in March 2020, South Africa had roughly 93,295 acute care beds available in private acute care facilities $(41,954)$ and in public regional, tertiary, and academic hospitals $(51,341)$. The total number of ICU beds (i.e., those potentially with ventilators) stood at 3,318, with 2,140 in the private sector and 1,178 in the public sector. High care beds, which have similar nurse intensity to ICU beds and access to oxygen but not necessarily ventilators, stood at around 2,722 in March 2020, with 1,640 in the private sector and 1,082 in the public sector (Table 9.5).

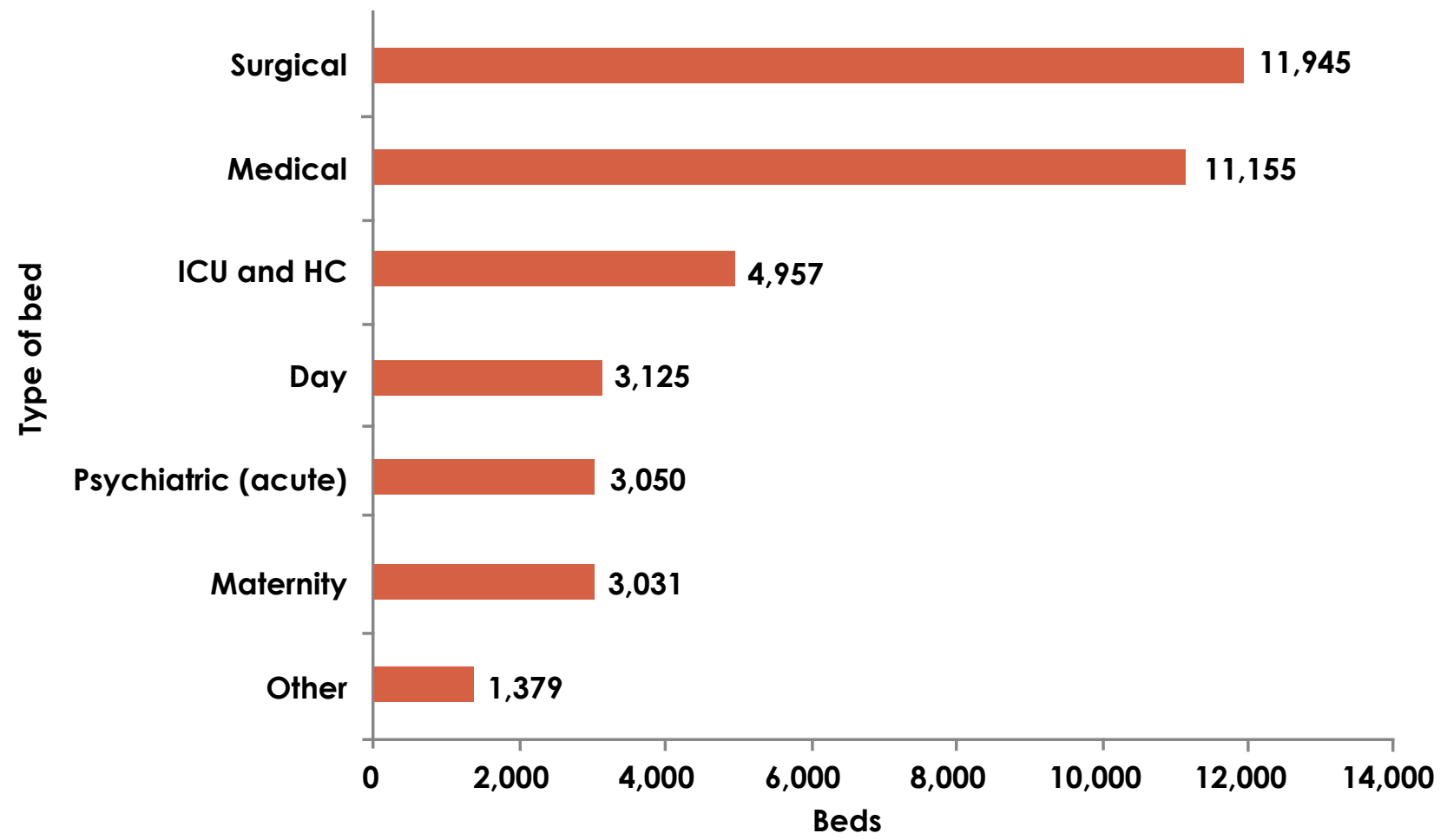

Figure 9.4: Private sector hospital beds by bed type (2016 estimate)

Source: Alex van den Heever, 2018 
Table 9.5: Acute bed availability in the South African health system in March 2020

\begin{tabular}{|c|c|c|c|c|c|}
\hline Sector & Province & Acute beds & $\begin{array}{c}\text { Total critical } \\
\text { care }\end{array}$ & ICU beds & HC beds \\
\hline \multirow{9}{*}{ Private } & EC & 2145 & 159 & 93 & 66 \\
\hline & FS & 2708 & 310 & 114 & 196 \\
\hline & GP & 18094 & 1871 & 1132 & 739 \\
\hline & $\mathrm{KZN}$ & 6152 & 516 & 305 & 211 \\
\hline & LP & 741 & 44 & 28 & 16 \\
\hline & MP & 1846 & 97 & 63 & 34 \\
\hline & NC & 918 & 34 & 27 & 7 \\
\hline & NW & 2230 & 197 & 87 & 110 \\
\hline & WC & 7119 & 552 & 291 & 261 \\
\hline \multicolumn{2}{|l|}{ Private Total } & 41954 & 3780 & 2140 & 1640 \\
\hline \multirow{9}{*}{$\begin{array}{l}\text { Public } \\
\text { (regional, } \\
\text { tertiary } \\
\text { and } \\
\text { academic) }\end{array}$} & EC & 6029 & 241 & 110 & 131 \\
\hline & FS & 3394 & 184 & 109 & 75 \\
\hline & GT & 13170 & 819 & 330 & 489 \\
\hline & $K Z$ & 13097 & 386 & 273 & 113 \\
\hline & LP & 3501 & 69 & 34 & 35 \\
\hline & MP & 1579 & 51 & 25 & 26 \\
\hline & NC & 929 & 34 & 21 & 13 \\
\hline & NW & 3251 & 81 & 54 & 27 \\
\hline & WC & 6391 & 395 & 222 & 173 \\
\hline Public Total & & 51341 & 2260 & 1178 & 1082 \\
\hline Grand Total & & 93295 & 6040 & 3318 & 2722 \\
\hline
\end{tabular}

Source: Data from (National Department of Health, 2013; Alex van den Heever, 2018) 
While some attempts were made to expand capacity to deal with the peak periods of demand through the construction of field hospitals, there is no evidence that the number of staffed beds changed significantly during the peak demand periods (July/August 2020). The highest recorded number of Covid-19 admissions to both the public and private sectors occurred on 1 August 2020, with total admissions at 8,310, of which 1,520 required critical care (National Institute of Communicable Diseases, 2020). Of those requiring critical care, 799 required ventilation. While there is some question regarding the accuracy of Covid-19 admissions in public hospitals outside of the Western Cape and the private sector, these numbers appear broadly consistent with the tracked new infections. Total Covid-19 admissions, therefore, never exceeded the combined capacity of the public and private systems, even after accounting for non-Covid-19 occupancy. Many hospitals in both the public and private sectors cancelled elective surgery from April through to August to ensure that critical care beds would be available for Covid-19 patients.

Out of all the provinces, only the Western Cape entered into an agreement with the private sector to access critical care beds if required. Ultimately, the agreement was not required as access to oxygen was expanded within the public sector and only a small number of public patients accessed private beds in the Western Cape.

\section{Comparative healthcare systems}

Having described the current healthcare system in South Africa, we now turn to describing briefly some of the different ways in which healthcare is organised elsewhere around the world.

Most of Europe, the United Kingdom, Canada, and a number of other countries have what is often described as 'universal healthcare', where everybody is able to access more or less the same services without distinction. The precise details of the organisation differ considerably from country to country, but the end result is much the same. The United Kingdom, for example, has a general taxfunded system, where doctors and hospitals are reimbursed directly for their services by local trusts (government organised decentralised purchasing units) using funds raised through taxes. In France, as another example, doctors and hospitals are in part reimbursed through the government, or the

A number of countries have what is often described as 'universal healthcare', where everybody is able to access more or less the same services without distinction. Sécurité Sociale, and in part by supplemental insurance whose terms are largely defined by legislation, with minimal co-payments by the patient. The system is set up so that everyone receives largely the same basic coverage. The organisation of services (often referred to as purchasing) is organised largely at local levels. In Germany, the Netherlands and Belgium, purchasing occurs 
through regulated markets by private mutual funds supported by government pooling mechanisms (to account for differences in income and the need for health services).

In other countries, most notably the United States, healthcare is largely organised by less regulated private markets for insurance and healthcare provision. Social insurance arrangements involve Medicare, which provides healthcare to lowincome groups, and Medicaid, which provides coverage to people over the age of 65. For people falling outside of the means test for Medicare and below the age of 65 , coverage is precarious if not part of a group health insurance arrangement offered through an employer. The end result is highly unequal access to healthcare and, also, substantial inequality in quality of the healthcare received. While the United States leads the world by far in spending per capita on healthcare, the indicators of the results do not lead the world. For example, infant mortality (defined as dying before the age of one year) is 5.9 per 1000 live births in the US, whereas the corresponding figures for a sampling of other countries are as follows: 2.2 in Japan; 3.1 in Germany; 3.3 in France; 11.65 in China; 38 in South Africa; and 76 in Nigeria.

Life expectancy provides another useful performance indicator. Here are some numbers from a few selected countries:

\begin{tabular}{|l|l|}
\hline Hong Kong & 84.7 \\
\hline Japan & 84.5 \\
\hline Italy & 83.6 \\
\hline France & 82.5 \\
\hline UK & 81.2 \\
\hline Costa Rica & 80.1 \\
\hline US & 78.9 \\
\hline Cuba & 78.6 \\
\hline Mexico & 75.0 \\
\hline China & 72.9 \\
\hline Russia & 72.4 \\
\hline Rwanda & 68.7 \\
\hline India & 69.4 \\
\hline Senegal & 67.7 \\
\hline South Africa & 63.9 \\
\hline Zimbabwe & 61.2 \\
\hline Nigeria & 54.3 \\
\hline Central African Republic & 52.8 \\
\hline
\end{tabular}

Source: United Nations 
Currently, a vigorous debate is taking place in the United States on how best to organise healthcare, with some advocating a system closer to the European model and others advocating maintaining the status quo. Former US President Barack Obama implemented reforms, expanding coverage to individuals falling outside of employer-based group coverage. The current debate centres on expanding Medicaid to provide default coverage for people unable to access proper insurance coverage through voluntary health insurance arrangements. Unregulated voluntary health insurance markets structurally fail to provide proper coverage to people with pre-existing medical conditions and those with inadequate incomes. Opposition to an expansion of Medicaid comes from private commercial health insurers, who would lose significant business to public coverage. Healthcare was one of the key issues of the 2020 US presidential election.

Many third world countries have a hybrid system

Throughout the world, how to organise healthcare will continue to be a vigorous subject of debate. with a minimal public offering for the majority at no or minimal charge, and another parallel private system for the middle and upper classes, which is financed by insurance premiums for those who can afford them.

Throughout the world, how to organise healthcare will continue to be a vigorous subject of debate. As a general trend, healthcare costs are rising faster than inflation as new treatments are becoming available. Another challenge is the inequality with urban and rural areas, which have a lack of doctors and less advanced care. 


\section{Covid-19 and Secondary Education}

Adolescence is derived from the Latin word 'adolescere' which means 'to grow up'. When adolescence begins and when it ends varies by individuals, societies and time periods. According to the World Health Organization, adolescence is the period from 10-19 years, while another definition is that it is the period from puberty to legal adulthood, depending on the age of majority in a country. This period of transition from childhood to adulthood is one of the most dynamic and influential periods of biological, physical and psychosocial development. 
Major physical and cognitive changes take place during adolescence, including changes in hormones and sex organs; rapid physical growth in terms of height and weight; and, importantly, there are significant changes in brain organisation and in the structure and function of thinking, reasoning and information processing. Two brain regions that undergo significant changes and development during adolescence are the limbic system and the pre-frontal cortex. The limbic system is responsible for regulating sleep, for emotion processing and for pleasure seeking. The pre-frontal cortex, on the other hand, is responsible for what are called 'executive functions'. These include planning for the future and impulse control. Importantly, pre-frontal cortex changes take place later in adolescence than those in the limbic system, sometimes tipping people into taking risks that they cannot yet process. As they develop, adolescents develop improved abilities to think abstractly, to manage emotions and to reason in more effective ways.

Coronavirus disease (Covid-19) has affected adolescents directly and indirectly. Beyond the possibility of becoming infected or ill, the mental and socio-emotional well-being of many people has been negatively affected by the pandemic, including adolescents. This is the time when patterns of behaviour that either offer protection or pose harm to one's health and well-being (in the short- and longterm) are established. At the same time, interventions that can support adolescent health and well-being can be especially beneficial during this period, and their absence can come at a cost. Not all experiences of adolescents during crises

For many young people living in South Africa, finding employment is already a difficult process. like this pandemic increase their psychological distress or have negative effects on their health and well-being. In important ways, adolescence is also a time of adaptation and learning, with many experiences (even difficult ones) actually fostering resilience. Some level of adversity that is not extreme or prolonged, and occurs in the presence of supportive others, can build resilience and be part of the learning process that enables adolescents to better manage their emotions, thoughts and behaviours.

Understanding how adolescents' daily lives have been affected by the pandemic, can guide support and assistance necessary to maintain and promote health and well-being. What are some of the major developmental tasks of adolescence? How has Covid-19 impacted on these? For teachers, understanding the reality of what learners are going through at this time, and how a normally tumultuous period of life is being exacerbated by Covid-19, may guide teachers to a deeper level of patience and understanding. It may also help for teachers to recall when they or their children were adolescents, what their experiences were, and what would have been helpful from their teachers at that time. 


\section{Planning for the future}

The last three years of secondary school are acutely focussed on the future-on getting the necessary assistance and guidance from teachers that will contribute to keeping learners in school at an incredibly stressful time, to achieve the marks that will allow young people to complete schooling successfully, to secure entry to tertiary education or training that is of interest to them, and, eventually, to secure a well-paid job.

\section{Covid-19 has made everything more complicated}

On the one hand, schooling has been massively interrupted. For those in Grade 12, writing final exams is stressful enough. Months of school closures, limiting opportunities to keep abreast and consolidate learning, has caused anxiety and worries. Will I have to leave school because of financial pressures on my family or repeat a Grade because I can't catch up? Will my marks be sufficiently good for my future plans?

\section{Where is life heading?}

It is clear that the economic impacts of Covid-19 are going to be felt for a long time. For many young people living in South Africa, finding employment is already a difficult process. After Covid-19, it may be even more difficult. Will I graduate from secondary school, get into a degree or training programme, find a job, be able to help my family?

\section{School completion}

For adolescents in Grade 10-12, a key life task is completing sufficient schooling and securing results that can open up avenues for bettering their lives. For many
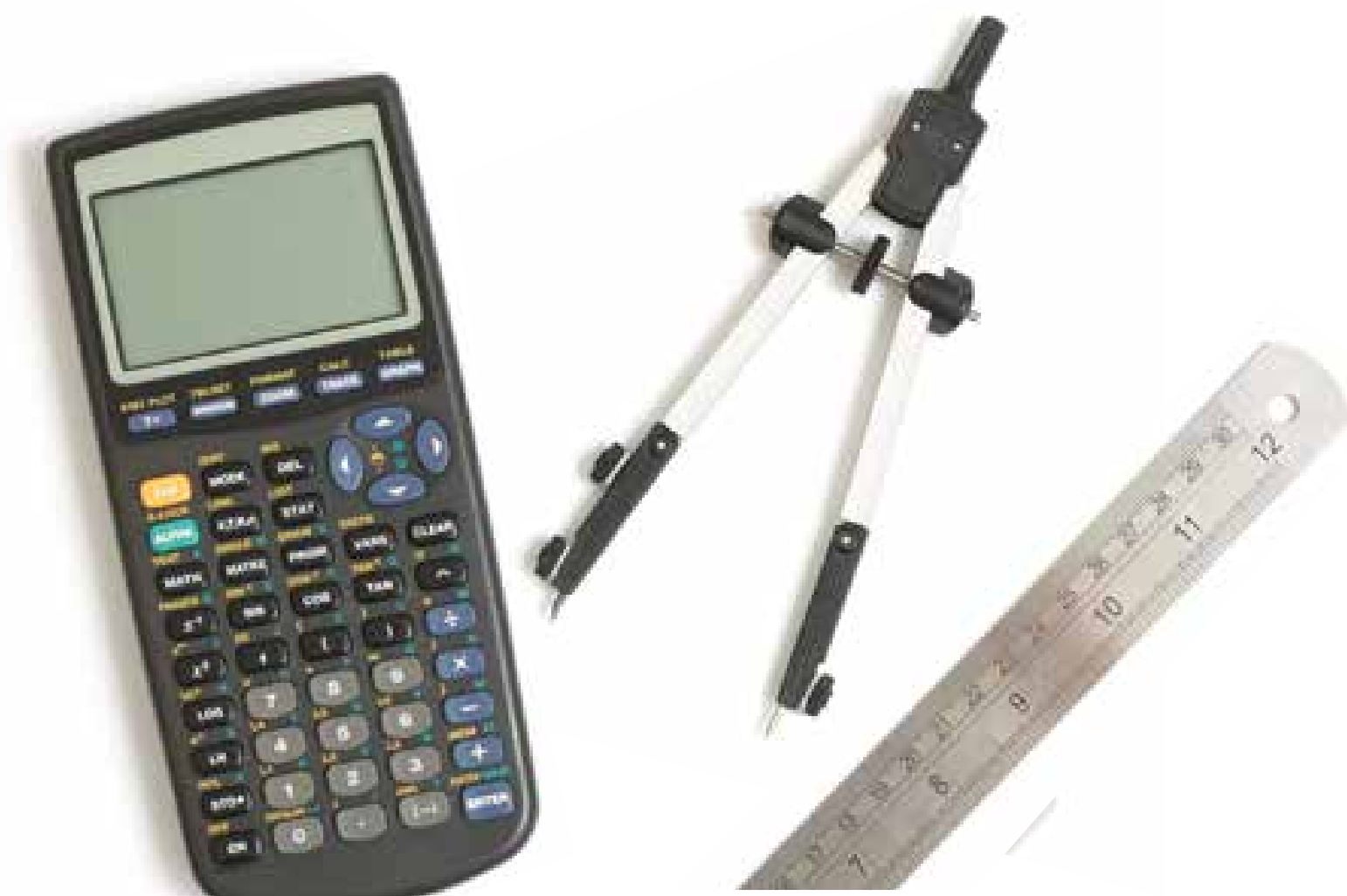
learners in South Africa, falling behind in school work, repeating a grade, lack of financial support, unplanned pregnancy and other stresses can severely challenge this goal. With ongoing homework support from teachers and family, learners hope that they can secure good marks in the final years of school. Missing months of teaching, not having a smart phone or computer and not being able to afford data charges can lead to falling behind on key steps in learning, and many feeling that they will never be able to catch up the work. Some may, in fact, not be able to return to school due to social and economic hardships.

\section{Developing agency}

A key developmental task of adolescence is developing a sense of agency in the world, a conviction that, with hard work and commitment, one can achieve a measure of control over one's future. With prolonged school closure, worries about potential future closures, absentee teachers due to illness or high risk for becoming infected, there is a strong likelihood that many learners may become discouraged by what appears to be the many things outside of their control. This may result in a sense of hopelessness, which challenges young people's developing sense of agency and a belief in their own capacity to determine their own future.

\section{Excitement and impatience}

Adolescents are on the cusp of an independent life and, for many, the desire to leave home and explore the world, develop new relationships and have new experiences is a profoundly exciting prospect. At the same time, many adolescents are impatient and want all these things to happen now and not to wait. Covid-19 has interrupted much of this, put it on hold, and put up what, at times, feels like insurmountable obstacles in the way of these plans. Many adolescents are feeling increasingly stuck and are impatient to 'start their lives'.

Separation from parents and caregivers. The developmental task of becoming less dependent

At the same time, many adolescents are impatient and want all these things to happen now and not to wait. on parents and caregivers is another key task of adolescence. Covid-19 has stopped this process in its tracks. Far from 2020 being a year when adolescents progressed along a path of independence, strict lockdown imposed exactly the opposite. Many adolescents have been forced to be with parents and family 24/7. Adolescents are biologically and emotionally geared to connect with the world beyond their families. These restrictions on their movement and changes to their routines with friends, school and extracurricular activities limit their ability to make decisions about their life, and the challenges happening around them can lead to feelings of powerlessness and frustration. 
Peer group and friendship. Perhaps the single most important activity during adolescence is deepening friendships and exploring peer relationships. These are linked to growing independence from parents and caregivers, and the intimacy of relationships with parents and family now becomes focussed on peers-both romantic and friendly. Covid-19 and South Africa's 5 months of lockdown have interrupted natural processes of developing or strengthening these peer relationships. For many adolescents, the most difficult parts of lockdown and social distancing have been not attending school and not being able to see their friends to deepen these relationships.

\section{Risk taking}

Adolescence is also a time of risk taking, in both good and bad ways. Risk taking is linked to exploration and innovation, developing a sense of agency in the world and developing a sense of one's own limits. Testing limits through risk taking contributes to the development of a deeper sense of what we are able to cope with, complete, or avoid later in our lives. Covid-19 and lockdown have upended these completely normal processes. The isolation of lockdown, the uncertainty about when it will end or when it will be reinstated, coupled with the deep sense of urgency within many young people to reconnect with friends and their peer group and to build further peer networks, may result in many adolescents taking added risks and, thereby, putting themselves and others at risk of Covid-19 transmission.

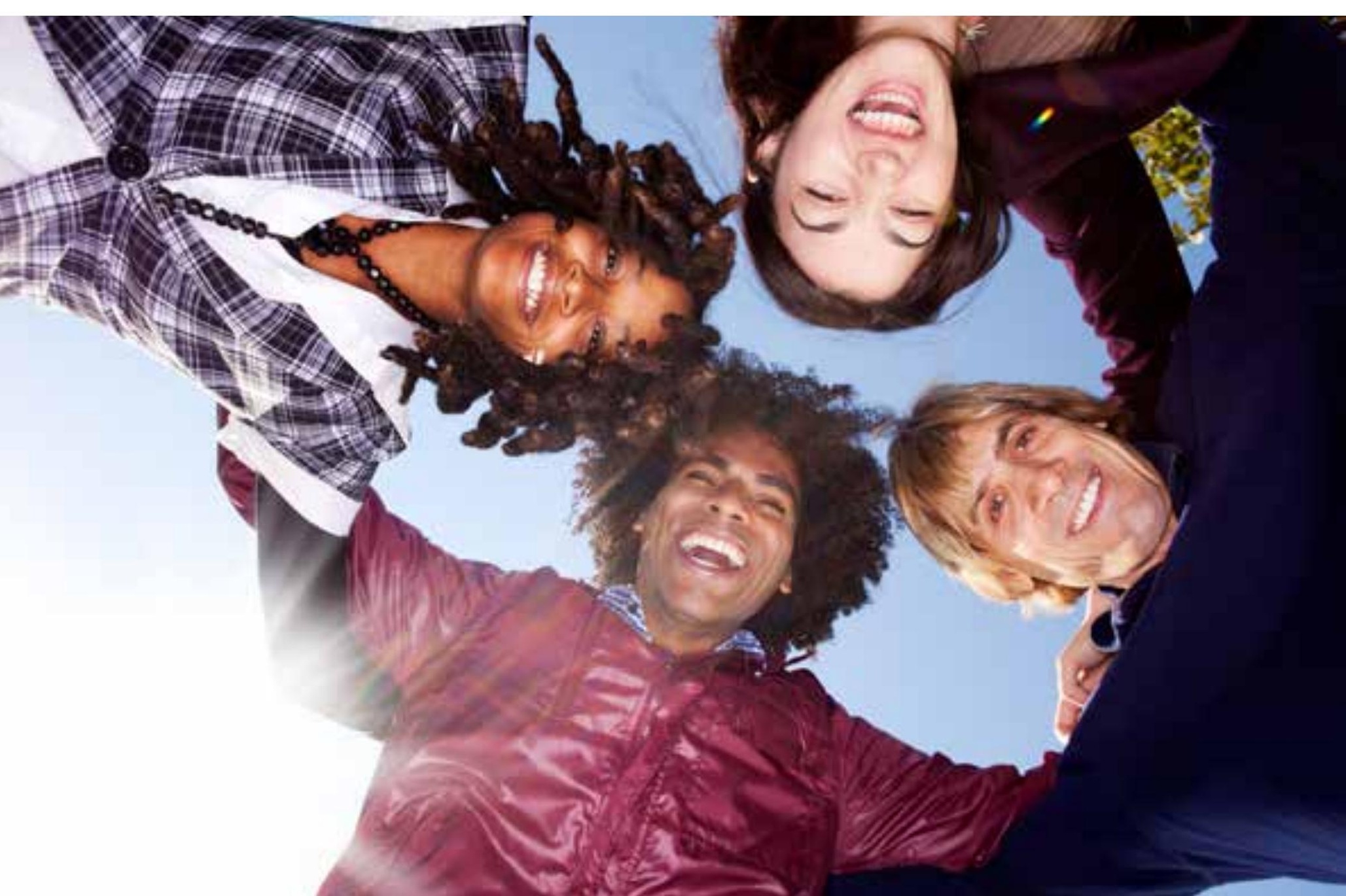




\section{Self and other}

A prevailing myth about adolescence is that it is a time of 'narcissism'-that adolescents are only interested in themselves and have little regard for others. This is far from the truth. Adolescents are actually highly focussed on others-largely their friends, peer group and nascent romantic relationships. They are profoundly focussed on the 'other'. What might account for the myth? Developmentally, an important task of adolescence is that of developing a deeper sense of self, a sense of self separate from caregivers. Caregivers and families often experience this as a rejection. Additionally, one of the consequences of developing this self is an increasing tendency to compare ourselves with others. Sarah-Jayne Blakemore, an expert on the adolescent brain, describes how, together with a growing comparison of self with others, the realisation arises that others are doing similarly-and making judgements about us. This makes adolescents appear acutely self-aware and, at times, 'narcissistic'.

Focusing on oneself, taking some risks to push limits to understand the world better, separating from parents and caregivers in order to develop an independent identity, and beginning to take the leap outwards into the world are utterly normal features of adolescence. But Covid-19 has put adolescents in a double bind. They are being forced into lockdown with the very people they are supposed to 'separate' from. They are being isolated from their peer group and the context where they can explore friendships and intimacy. And, all the while, they know that they are less likely to become infected, ill or to die from the virus than others. Because adolescents can carry the virus and infect those who are vulnerable around them, adolescents have to

Risk taking is linked to exploration and innovation, developing a sense of agency in the world and developing a sense of one's own limits. change their behaviour in order to protect others. They are being asked to adopt a set of behaviours that are essentially almost entirely 'other focused', protecting others (mostly older adults) from something that will in all likelihood not seriously harm adolescents themselves. Finally, in the South African context, adolescents are frequently the primary caregivers of siblings or their own children and are very keen to protect their friends.

\section{Time of heightened worries}

There is an intensity to the emotions and experiences of adolescence that are rarely felt again in one's life. First love, falling out with one's closest friend, and failing to achieve a cherished dream are often felt by adolescents as catastrophic. Linked to this are worries about loved ones, about parents or grandparents becoming ill and dying. Many adolescents in South Africa are cared for by elderly grandparents, or parents that are HIV+ or have co-morbidities such as diabetes and hypertension, making them more vulnerable to serious illness due to Covid-19. 


\section{Recommendations and a way forward}

One of the few bright spots in the pandemic is that it is global. And while, of course, some countries have been more affected than others, no one in the world is untouched. Know $100 \%$ that no one is alone in this. There are millions of adolescents around the world who are feeling anxious, concerned for the future, and feeling lonely and isolated. It is important for adolescents to reach out to their classmates and friends and tell them that they are thinking about them. Receiving a caring response from a friend is invaluable. Maintaining connection and engagement with others is possible and crucial to our well-being.

Routine in times of uncertainty helps us all and is vitally important. Routine creates a sense of normalcy amidst a world that has become increasingly confusing.

\section{Physical activity and exercise}

It is well established that exercise releases endorphins and is good for our mood. Exercise, and walk with your friends. This can be done safely and within the required regulations and is another way to maintain social connections.

\section{Adolescent and youth led innovation}
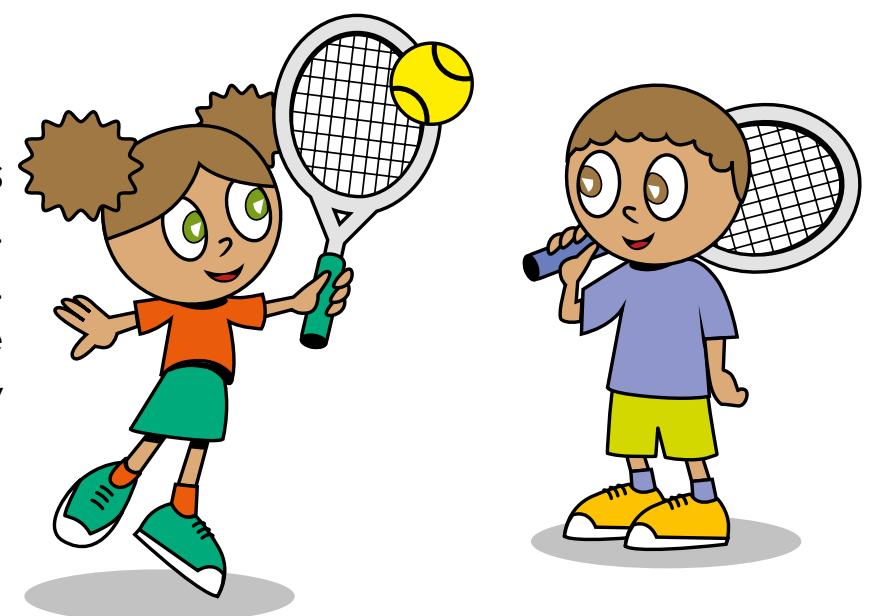

Adolescents are powerful health promoters. Engaging them in campaigns to encourage mask wearing, hand washing and social distancing is important for them and can make a meaningful contribution to societal change. Adolescents are important agents of change and can be the most effective message carriers among their peer groups but also within their households and communities.

For adolescents who are feeling despondent or 'stuck', recall all the things you have accomplished under difficult circumstances, how much your family and friends believe in you and wish you well, draw on your faith, and try to keep moving forward.

\section{Lots of people are anxious and worrying now}

You are not the only one worrying. Remember that the job of parents and educators during this time is to carry as much of the worry as they can. Their job is to let those in their care explore, learn, and create. Lots of people are thinking about you and let them do some of the worrying for you. If feelings of anxiety and worry are becoming overwhelming, it is important to find someone you trust to talk to and express those feelings and to ask for help. 


\section{Creativity}

Adolescence is a time of exploration and deep creativity. Use this opportunity to explore this side of yourself. Draw, write, sing, dance and imagine. Creativity builds resilience.

\section{Harnessing the power of social media}

The most powerful agents in the promotion and protection of mental and physical health amongst adolescents are other adolescents. Harness the power of social media as a healthy way to connect with your peers who are sharing some of your feelings and experiences.

\section{Patience and keeping learners in mind}

A key job of teachers during this time is to be mindful of what the learners in your care are going through. Of course, you have concerns for your own health and for that of your family. But the learners in your classes have seen the world they know come to a standstill and, for many, the future has become increasingly uncertain. For some learners, teachers are their hope for the future and their passport out of sometimes difficult circumstances. Reach out to them, remind them that you are thinking of them, offer support in any way you can, and remember how difficult so much is for them right now. Be patient. 


\section{CHAPTER 11}

\section{Covid-19 and the Economy}

The Covid-19 crisis has had a devastating effect on economies around the world and South Africa is no exception. The various lockdown measures have reduced economic activity, causing many to lose income and their jobs. Many businesses have struggled to remain solvent, and many have failed as aresult of the pandemic. Governments have endeavoured to provide support to affected workers and businesses. However, the needs are enormous. Governments in less wealthy countries have found themselves limited in meeting these needs owing to their limited abilities to borrow on the international bond market. Governments around the world have struggled to find the right balance between preventing the spread of the disease through public health measures and avoiding an excessive negative impact on the economy. This chapter describes some of these issues with an emphasis on the South African situation. 


\section{Context}

On 26 March 2020, South Africa initiated a severe lockdown as its primary intervention to interrupt the transmission of the SARS-CoV-2 virus. The period of the lockdown was initially set for 21 days but then extended until 31 April 2020. A rough estimate put the lost gross domestic product (GDP) at approximately R 13 billion per day for what is now referred to as the Level 5 version of the lockdown. During this period both the supply (production of goods and services) and demand (consumption of goods and services) sides of the economy were largely closed down. The exceptions were producers of essential public goods and services.

Less restrictive lockdowns, beginning from 1 May 2020, involved a partial opening of the economy. However, major sectors remained closed until the shift to Level 2 from 17 August 2020. In total, a large part of the economy remained closed for a period of five months. Current estimates put the overall annualised loss in economic output or GDP expected for 2020 at around $8.2 \%$. Current estimates also put lost employment at around 2-3 million, with some sectors likely to remain below their full potential for at least the medium-term (i.e., a period of around three years).

The consequences flowing from the decision to pursue a generalised lockdown will, in all likelihood, be with South Africa for many years to come.

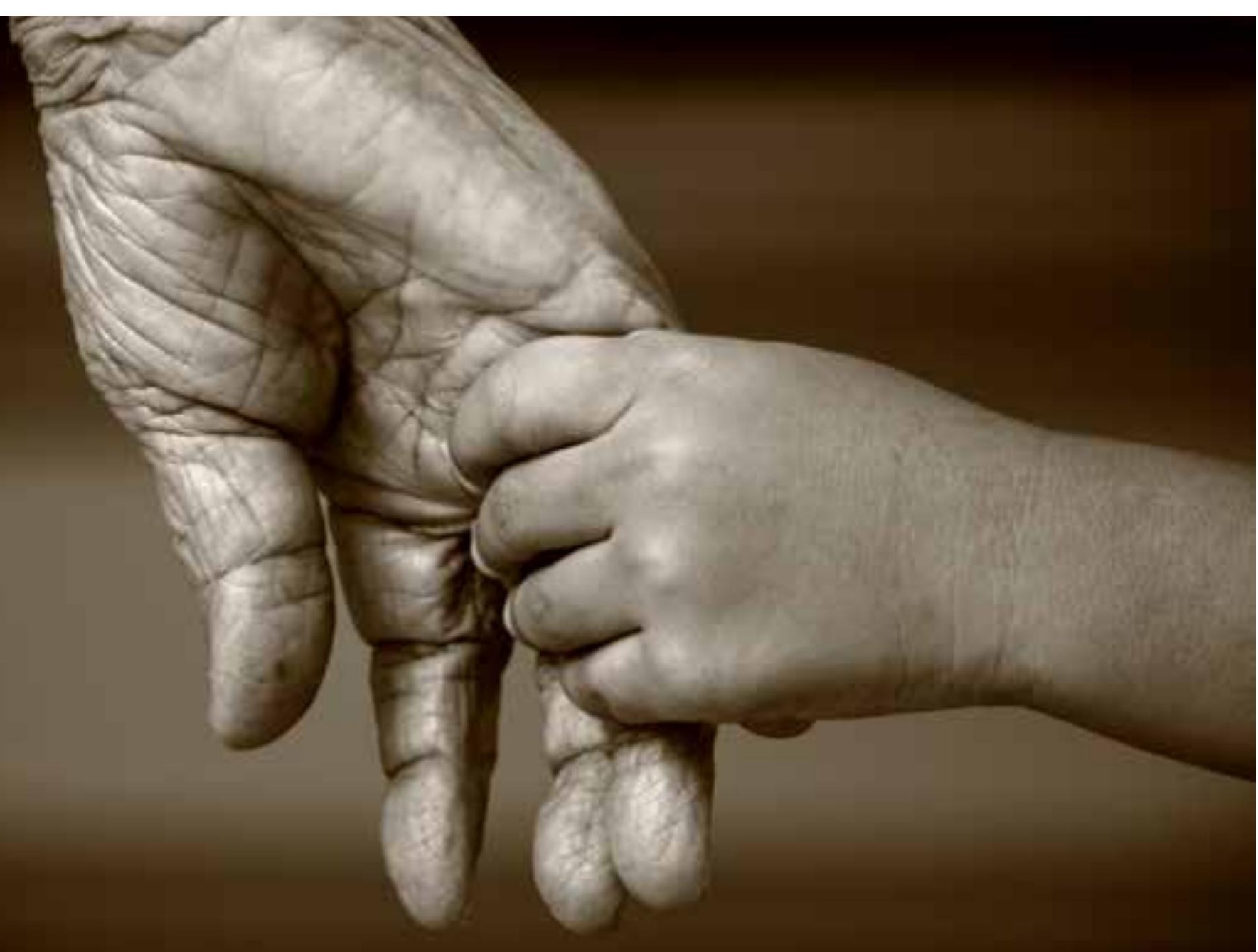


Use of a generalised lockdown as the primary non-pharmaceutical intervention can largely be attributed to the absence of alternative public health measures that could be implemented at scale at sufficient strength in time to interrupt the spread of the local epidemic. Important amongst these alternative measures are testing, contact tracing and quarantining.

Countries slow to respond with adequate measures faced raging local Covid-19 epidemics that could not be contained using measures other than crude lockdowns. In contrast, South Africa was at a very early stage of the epidemic in March 2020 and could, arguably, have considered alternative measures but chose not to.

\section{Social and economic implications}

The consequences flowing from the decision to pursue a generalised lockdown will, in all likelihood, be with South Africa for many years to come, as the collateral effects are wide ranging. These effects are also likely to prove complex, with their social and economic features difficult to disentangle due to large-scale loss of livelihoods.

Three broad areas of impact can be identified.

Firstly, there are the social effects arising from immediate loss of employment and livelihoods. Those affected with limited savings and assets are the most vulnerable and would require immediate support of some form to survive the temporary period of hardship. However, where longer-term harm is done to livelihoods, deeper interventions are needed. The question is whether South Africa has the institutions that are able to address both the temporary and longer-term needs of society.

Secondly, there are the economic effects which can arise from two sources. The first results from the lockdown and the simultaneous closure of businesses, the interruption of supply chains and the loss of demand for products.

The second arises from reduced demand for service-related businesses, such as medical practices, hospitals, hotels and restaurants, where a fear of infection is presumed to be high.

Thirdly, there are severe consequences for public finances, which involve three main shocks.

The first is the immediate loss of tax revenue resulting primarily from the economic impact of the lockdown.

The second is the higher expenditure requirement to support vulnerable households and businesses during the period of the lockdown-and potentially beyond. 
The third is that government's ability to finance the increased deficit is compromised by distressed financial markets, together with a loss of confidence in its ability to repay its fast-rising, accumulating debt.

\section{Social effects}

The social implications of the Covid-19 pandemic are complex and closely tied to the economic consequences. Prior to the pandemic, the socioeconomic context was already characterised by structural unemployment, inequality and poverty. The expanded unemployment rate', which stood at $39.7 \%$ in the first quarter of 2020 , increased dramatically to $42.0 \%$ in the second quarter. The declines in employment affected both formal and informal sector settings.

Giventhe dependencyonemployment to distribute incomes, the high levels of structural unemployment explain South

As yet uncertain, however, are the implications for international and domestic economic growth arising from the substantial stimulation packages initiated in the more industrialised economies.

Africa's extraordinary levels of income inequality, with South Africa at the bottom of all the international league tables. This unequal distribution of income also serves to skew domestic economic and industrial development, as economic demand is concentrated in the hands of a relatively small number of high-income households. What they choose to buy, consequently, shapes what we produce or don't produce. Through the employment losses, this underlying economic misalignment may, therefore, further embed the historical distortions in income distribution, unemployment and economic diversification.

Many countries institutionalise healthier distributions of income through welldeveloped systems of social security, which include social insurance (contributory social security), social assistance (cash grants), and free social services (healthcare; education; social work; care support for the elderly and disabled; social housing; adult skills development and efficient job placement). These systems diversify the sources of income for households beyond the reliance on incomes from work or assets (which only benefit very high income earners). In this way, many households are destressed while simultaneously shaping

1 This includes those aged 15 to 64 years not employed and actively seeking work, as well as discouraged workers (i.e., those who want to work but have given up hope). This is a better reflection of unemployment than the official definition which excludes discouraged workers. 
a healthier distribution of consumption, with important implications for the richness and diversity of the domestic economy.

An enhanced social security system, however, cannot be implemented in the shortterm, and even an accelerated programme of institutional and benefit reform could take well over 15 years. The ability of government to enhance the distribution of income has, however, also been compromised by the fiscal implications of the Covid-19 crisis, which has left public finances in a distressed state likely to last for at least the medium-term. Nevertheless, given the positive economic effects of improved distributions of income, incremental advances on this front appear to have merit and, given the acute nature of the social crisis, will need to be considered regardless of the fiscal position.

\section{Economic effects}

The simultaneous closure of both the supply and demand sides of the economy on the scale seen in the lockdown is unprecedented for South Africa, and the full implications are only likely to materialise over time.

\begin{tabular}{r|c|} 
Overall & \multicolumn{2}{|c}{$8,2 \%$} \\
\cline { 2 - 2 } Personal Services & $4,3 \%$ \\
General Government Services & $0,2 \%$ \\
Real Estate and Business Services & $3,4 \%$
\end{tabular}

Transport, Storage, and Communication

Wholesale and Retail Trade, Hotels and Restaurants

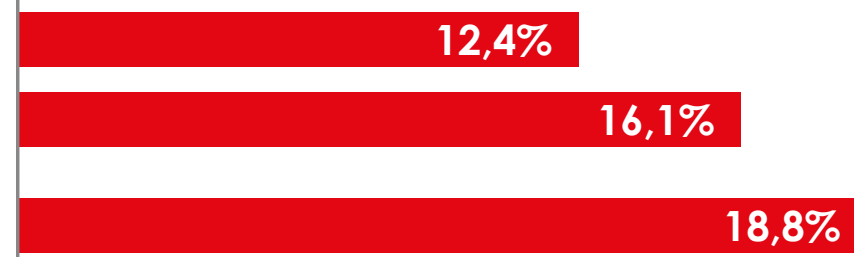

Construction

Electricity and Water

Manufacturing

Mining and Quarrying

Agriculture, Forestry and Fishing

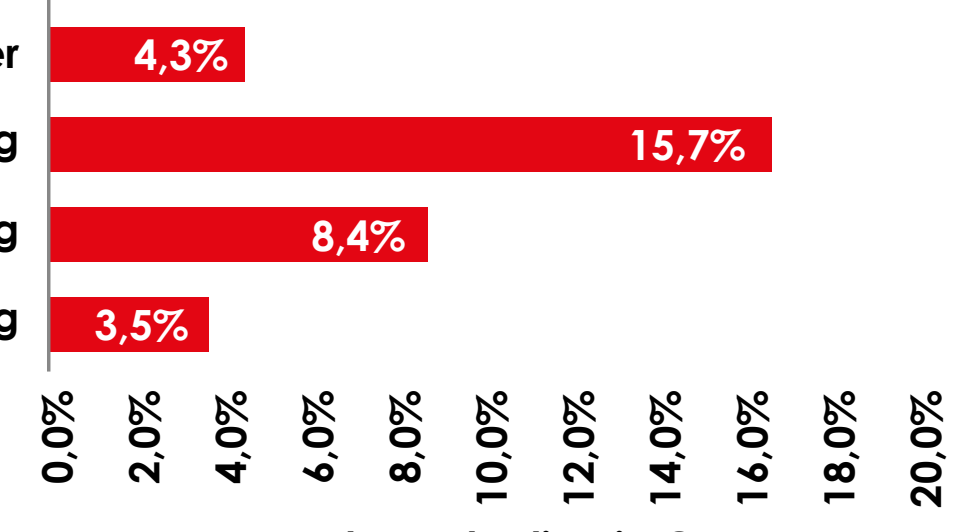

Percentage decline in GDP

Figure 11.1: Estimated decline in GDP by sector of the economy 
Government also initiated the Temporary Employer-Employee Relief Scheme (TERS) through the Unemployment Insurance Fund (UIF) to support the incomes of workers remaining in employment to keep businesses viable. As the UIF does not have a member registry (it only has an employer registry), it needed to set up an application process to vet applicants for the support. A combination of weak systems and inhouse corruption contributed to slow uptake and delivery failures. Government's failure to timeously initiate economic support programmes has, however, contributed to the severity of the economic downturn and to the possible protracted nature of a recovery.

Four of South Africa's most important sectors are now expected to see declines in excess of $10 \%$ (Figure 11.1), with the hardest hit being Construction with a decline of 18.8\%; Wholesale, Retail trade, Hotels and Restaurants with a decline of $16.1 \%$; Manufacturing with a decline of $15.7 \%$; and the Transport, Storage and Communication sector with a decline of $12.4 \%$. The Financial services sector, in contrast, faces a decline of only $3.4 \%$, with Government showing virtually no decline.

The financial sector has proven quite resilient in the face of the 'lockdown' and has in many

There are entities crucial to the South African economy that need to be restructured and reconceptualised. ways contributed to supporting the viability of private industry in general. This was made possible through bank-initiated loan restructuring arrangements and even strategic interventions during the lockdown to mitigate the epidemic. Given the pivotal position of the financial services industry, any failure by them to take mitigating action will invariably have far reaching collateral implications for many industries.

An instance of poor conduct by the financial sector has seen the short-term insurance industry act in unison to avoid paying out business interruption policies taken out by the hospitality industry. In this way, they contributed to failures in particularly vulnerable sectors, despite contractual obligations to provide support. Pre-Covid-19, the hospitality industry contributed around $8.5 \%$ to GDP and was the second-most important export sector (inbound tourism). Instead of paying out on policies, insurers opted to follow court processes to decide on liability, despite apparently clear policy wording that required they pay out 
when business interruptions resulted from notifiable infectious and contagious disease outbreaks within a 50 kilometre radius of the business. As a consequence, hundreds of small businesses in the hospitality industry are having to await the outcome of protracted court decisions before being paid out. This has significant direct and indirect implications for the economy. The former involve the affected businesses directly. The latter arise from the knock-on effects of downstream businesses and their employees and dependent families.

On the positive side, a combination of closed borders and the lockdown have necessitated that some businesses repurpose their output to serve new (domestic) markets. Many retail outlets and restaurants developed online ordering arrangements and direct-to-household deliveries. It is likely that much of this restructuring will be permanent and create employment opportunities, albeit at the precarious end of the job spectrum. Many of these changes could aid the localisation of manufacturing in South Africa and stimulate new industrial development. Were this to be combined with a smoothing of demand across all income groups, resulting from a progressively improving social security system, the effects could be permanent and support economic diversification. However, many of these gains are likely to occur only over the medium-term and are far from certain at present.

\section{Public finances}

Planned government expenditure has remained virtually unchanged for 2020 , with stimulus plans stated as worth R500 billion, mostly funded through budget restructuring rather than new financial commitments. This weak fiscal response is largely due to the substantial loss of tax revenue arising from the lockdown and the protracted closure of the tobacco and alcohol industries. It is projected that the deficit will rise to around $15.7 \%$ of GDP with reduced revenue of around R300 billion.

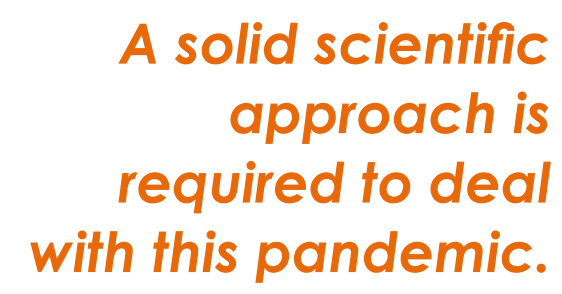

Whereas more industrialised countries are in a position to finance increased deficits through additional borrowing at low interest rates, South Africa went into the Covid-19 crisis with its debt downgraded to junk status. This influences the rate of interest government pays on new debt as well as the take up of any new bonds issued in the primary lending market (i.e., where government auctions off treasury bills to finance its deficit).

The South African Reserve Bank (SARB) was sufficiently concerned about liquidity in the South African capital markets during April 2020 to intervene by both lowering the benchmark interest rates and purchasing government bonds in the secondary bond markets. This was done to increase the capacity for private investors to take up government bonds in the primary market, so that government would have the funds to continue spending. Without this intervention, it was increasingly possible that 
government would not be able to raise the funds needed to finance its liabilities.

Given the downgrades by international ratings agencies, a concern arises concerning South Africa's ability to service the deficit, which has cumulatively increased to over R2 trillion (or around 65\% of GDP and steadily rising), as well as finance important public programmes, especially those central to revitalising the economy post-Covid-19.

It is for this reason that consideration has been given to the issuing of a special Covid bond at a special interest rate (which appears to have fallen through) as well as accessing some loan facilities at the International Monetary Fund (IMF) (\$4.3 billion on the government balance sheet. On the other hand, there are entities crucial to the South African economy that need to be restructured and reconceptualised. Their failures, arguably, derive from the governance arrangements permitting political appointments that encourage the entry of private interests into strategic public organisations.

Whereas employment in the public sector has not been impacted by the Covid-19 pandemic, the sectors from which it derives its funding have been badly diminished. What this implies is that productive industries have been severely depleted, while an inefficient public sector competes for the country's limited resources.

The fiscal position of the country is integrally tied to the performance of the economy. The economy will need to be reconstituted as a priority going forward. The success of such an outcome, however, depends on South Africa progressively improving its productivity.

\section{Conclusion}

The Covid-19 pandemic has already impacted heavily on South Africa's social and economic systems, increasing its fragility. Prior to Covid-19, South Africa's social and economic outcomes were already experiencing difficulties. The pandemic has rendered the situation more acute. The next three years will prove to be a decisive time in the history of South Africa.

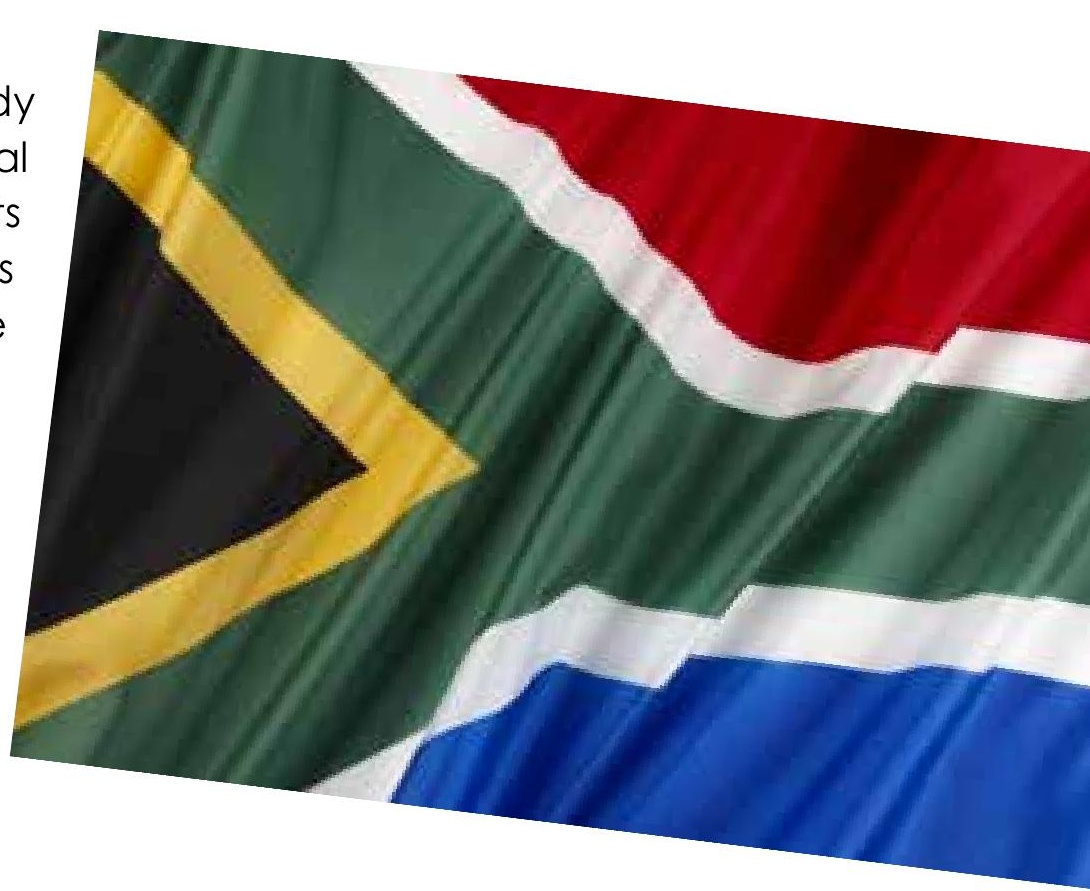




\section{EPILOGUE}

As this report reaches press, the Covid-19 crisis will still be unfolding. We remain in the midst of a long pandemic, which has taken over our lives, the South African and global economies, and our concern for the health of ourselves, our families and those around us. Here we have tried to present, as concisely and understandably as possible, the science underlying Covid- 19 and viruses more generally, the dynamics of how this disease and other infectious diseases spread, and impact on the healthcare system and the economy. We also discussed the sociological and psychological effects of the epidemic, focusing particularly on adolescent learners. We also presented some basic facts about how the South African health system is organised. In the coming years, debate will continue in South Africa, as elsewhere in the world, on how better to deliver healthcare, in a more efficient, equitable, and affordable way. It is important that this debate is not left to specialists exclusively, but rather that everyone takes part and understands the background and available options.

The devastating consequences of the coronavirus pandemic have been staggering. At time of writing (22 July 2021), there have been 14 million tests for the virus done, 2.3 million positive cases identified and 52111 deaths recorded in our country. Moreover, 2.1 million people have recovered from the virus. Since an initial hard lockdown at the start of the pandemic, the government relaxed lockdown restrictions to varying degrees over the course of the pandemic, which meant greater freedom of movement, the opening of more businesses and schools, the resumption of flights, and many interventions to kick-start the economy.

Knowledge matters, that science or

However, the danger is not yet over. In fact, many countries, including South Africa, have seen a resurgence of infections as lockdowns and other measures have been eased. This has led to debate and controversy about whether or not or how to re-introduce lockdown rules and other measures. The danger remains with us. We have been told that life can return to normal only once this coronavirus has been completely eradicated. And this can occur only when a vaccine against the virus has been widely distributed. A solid scientific approach is required to deal with this pandemic. A serious on-going concern is the effectiveness of the present vaccines against new strains of the virus, and new or modified vaccines may become necessary to counter resistant strains of the virus.

The crisis is a lesson to us all. We have been able to reach this situation of the easing of strict lockdown levels because many South Africans of all backgrounds have 
complied with governmental regulations, which included staying at home, regular hand-washing, social distancing and the wearing of masks. These very positive interventions were as a result of scientific thinking. Scientists first determined the source of the virus, isolated, and identified it as the coronavirus and studied the models of how it is spread. Based on these findings, scientists advised the governments how to proceed. This resulted in a mass worldwide campaign to test people for the virus, identify positive cases, perform contact tracing and, in the case of sick people with severe coronavirus disease, provide adequate hospital care, including ICU admission for the most serious cases. Given that several effective vaccines have now been found, the challenge now becomes massive production and distribution throughout the world.

\section{What does all of this tell us?}

It tells us that knowledge matters, that science or scientific research is absolutely crucial and, perhaps, as it yields positive results, it is the only hope we have of saving the world from the devastation this virus can cause. Decades of painstaking research in epidemiology, virology, clinical medicine, and laboratory medical research has brought the world to a point in which people's lifespans have been extended and huge pandemics have been eradicated through vaccines (for example mumps, rubella and measles) and medication (tuberculosis and anti-retrovirals for HIV-AIDS).

With the advent of primary healthcare and public health research, common sources of disease and infection such as contaminated water, poor sanitation, risky sexual practices, smoking, and alcohol abuse have been highlighted. Public awareness of such matters and other interventions have increased life expectancy and

We believe that the Covid-19 crisis has very clearly demonstrated the relevance of science in our everyday lives. quality. All these scientific achievements must be accompanied by good leadership and governance. Climate scientists also remind us that the risk of pandemics is exacerbated by climate change, brought on by our lack of care for our environments. We burn fossil fuels, discard all kinds of pollutants into our rivers and seas, chop down our forests, and, more generally, show little concern for our planet.

Since the outbreak of Covid-19 in late 2019, much has been learned about the virus and how to treat the disease better, thanks to the work of countless doctors and medical researchers. There still is no cure. For those most seriously afflicted, those needing hospitalisation, in some cases in an intensive care unit, the supportive care staves off the worst effects and buys time so that the body eventually can fight off the virus on its own. Due to a better understanding of the disease and efficacy of different treatment options, the number of fatalities per serious case has been brought down to about half of its initial rate. 
We would like to point out that Covid-19 will not be the last pandemic the world will ever face. Chapter 4 has shown how, throughout history, new diseases come along, often causing great disruption and destruction. Experts in public health have long pointed out this risk. Had their warnings been heeded, the world would have found itself better prepared to face this pandemic.

The Covid-19 has led to a surge in activity to understand, treat, and stem the spread of the disease. As expected, much of this activity has taken place within the traditional medical research and public health communities. But given the crisis, new actors have been inspired to explore how they can apply their expertise toward fighting Covid-19. Two examples in Africa, are: (1) the idea of pool testing, where mathematics has shown how a population largely Covid-19-free can be tested most efficiently-that is, with the fewest numbers of tests. Rather than testing each person individually, samples are combined and tested in pools, the combined results indicating who is positive. This technique, developed at the African Institute for Mathematical Sciences in Rwanda, is now being applied by researchers at the University of KwaZuluNatal. (2) The South African Radio Astronomy Observatory has successfully applied its expertise in engineering and manufacturing

The tremendous success of mass vaccination relies on more than a certain threshold fraction of the population being vaccinated, in order to benefit from "herd immunity". to produce low-cost ventilators for Covid-19 patients requiring artificial ventilation. Often progress in science and technology is inter-disciplinary, resulting when experts consider problems traditionally considered outside their range of expertise, often in collaboration with experts from other fields.

Finally, we hope that we may have inspired you to want to learn more about viruses, medicine, and epidemiology. Appendix A indicates some resources for further study. Often science and mathematics may seem like abstract school subjects, not so obviously relevant to our everyday lives. We believe that the Covid-19 crisis has very clearly demonstrated the relevance of science in our everyday lives. We also stress that it is often hard to foresee in advance which areas of science will be most important for tackling society's new problems and challenges. Often seemingly abstract and highly specialised areas of science, with time, become the foundations of the technology of tomorrow. Who would have predicted that the work begun by Jenner and Pasteur would have allowed such a decrease in childhood mortality? Who would have predicted that the work of James Clerk Maxwell, Michael Faraday, and others would have led to the omnipresence of electrical machinery and electronic devices in our everyday lives? In the 1850s, the British Chancellor of the Exchequer William Gladstone questioned Michael Faraday about the practical use value of electricity, and Faraday famously replied: "One day, sir, you may tax it." And that is exactly what happened. 


\section{APPENDIX A}

\section{RELIABLE SOURCES OF INFORMATION AND FURTHER READING}

Here, we provide a number of websites with reliable information to learn more about Covid-19 and the other topics covered in this report.

The following South Africa government websites provide a wealth of data about the prevalence and the spread of the disease in South Africa as well as more general information.

https://www.gov.za/Coronavirus

https://sacoronavirus.co.za/

https://covid 19.who.int/region/afro/country/za

https://www.nicd.ac.za/

The World Health Organization, which is part of the United Nations, provides worldwide Covid-19 statistics and other information about Covid-19, its treatment, and medical aspects.

https://www.who.int/emergencies/diseases/novel-coronavirus-2019

The following non-governmental websites are an invaluable resource for the latest data.

https://www.worldometers.info/coronavirus/

https://ourworldindata.org/coronavirus

These other websites are also excellent sources of additional information.

https://www.pasteur.fr/en/all-sars-cov-2-covid-19-institut-pasteur

https://www.cdc.gov/coronavirus/2019-nCoV/index.html

https://coronavirus.jhu.edu/

https://www.scientificamerican.com/interactive/inside-the-coronavirus/

The entrance, entertainment, and exit of the viral lifecycle is nicely explained in the blog.

https://rybicki.blog/2010/09/16/on-the-utility-of-pink-floyds-the-grand-viziersgarden-party-as-a-metaphor-for-virus-multiplication/ 
For more information about HIV/AIDS in South Africa and its treatment, see for example

https://www.unaids.org/en/regionscountries/countries/southafrica

https://hivinfo.nih.gov/understanding-hiv/fact-sheets/fda-approved-hivmedicines

https://sahivsoc.org/Files/Adult_ART_2017.pdf

https://unitaid.org/news-blog/south-africa-to-introduce-state-of-the-art-hivtreatment/\#en

For information about drugs against Covid-19 see

https://www.health.harvard.edu/diseases-and-conditions/treatments-forcovid-19

https://rebelem.com/the-recovery-trial-dexamethasone-for-covid-19/

and

https://www.contagionlive.com/publications/contagion/2020/august/using-il6inhibitors-to-treat-covid 19

The WHO website provides a wealth of information on immunization programmes, vaccines, and the diseases targeted.

https://www.who.int/immunization/programmes_systems/supply_chain/benefits_ of_immunization/en/

The news article

https://www.newscientist.com/article/dn4743-controversial-mmr-and-autismstudy-retracted/

provides an account of debate concerning the purported link between the MMR vaccine and autism, subsequently refuted by others and retracted by most of the authors of the original Lancet publication.

See

https://www.nature.com/articles/d41586-020-02501-3

for an account of the partial, not yet complete, eradication of polio thanks to the widespread use of the polio vaccine.

http://www.victorianweb.org/science/health/depaolo.html

gives an account of 19th century medicine. 


\section{APPENDIX B}

\section{MISINFORMATION, MYTHS, FAKE-NEWS, AND FABRICATIONS AROUND COVID-19}

We are flooded with information about Covid-19 from all kinds of sources-from social media, such as YouTube, Instagram, Twitter, Facebook, and various web pages; and also from newspapers, magazines, and governmental sources. We are presented with a confusing picture, with conflicting claims. It is sometimes not so easy to decide what to believe.

At the beginning of the pandemic some said that Covid-19 was "a hoax," or that it was "like the flu" and would "go away within a few weeks." Other sources claimed all sorts of miracle cures, including hydroxychloroquine and various herbal remedies. We also heard claims that seasonal effects would keep the disease away or make it disappear. Yet, months later, the disease still remains very much with us, with high numbers of people infected, hospitalised, and also deceased.

We live in the age where 'fake news' and 'alternative facts' abound. Care must be taken in deciding which sources to trust and which deserve to be doubted. These difficulties are compounded by the fact that much of the best information is tentative. As experts learn more, our best available understanding is being revised.

'Fake news' consists of deliberate misinformation or disinformation spread through various media platforms. Digital platforms currently lead in the spread of fake news-or 'yellow journalism'. Such news often gains momentum in social media and sometimes finds its way into the mainstream media as well. 
Information provided by local, internationally reputable institutions and medical experts about infection control measures and how the virus spreads competes with less reliable and conflicting information on social media. Myths associated with the spread and cure of Covid-19 in society often spread more widely and faster on social media platforms. Sometimes such 'fake news' and myths are spread by prominent members of society such as US President, Donald Trump, who claimed Covid-19 was just another flu and would be gone within just a few weeks. Such 'fake news' and myths can be very dangerous, because they can lead to over-complacency and to a laxity in the needed practices to curb the spread.

In South Africa, we once grappled with the question, 'Does HIV cause AIDS?' It took quite some time-some would say too long-for all involved to come to the realisation that HIV causes AIDS. This was a crucial step toward Myths associated with the spread and cure of Covid-19 in society often spread more widely and faster on social media platforms. curbing the HIV/AIDS pandemic through programmes promoting safe sex, testing, and anti-retroviral therapy. Accurate public understanding of health-related issues is crucial to preventing avoidable diseases and improving outcomes.

Here are a few examples of widespread myths:

Can you protect yourself from Covid-19 by injecting, swallowing, bathing in or rubbing onto your body bleach, disinfectants or rubbing alcohols?

These products are highly toxic and should never be swallowed or injected into the body. Disinfectants, bleach, and soap and water may be used to clean surfaces. This is an important prevention step in stopping the spread of the coronavirus. But never attempt to self-treat or prevent Covid-19 by rubbing or bathing with bleach, disinfectants or rubbing alcohol anywhere on your body. Effective hand sanitisers do have alcohol, but they are formulated to be safe for use on hands.

\section{Was the new coronavirus deliberately created or released by people?}

Viruses change over time, and from time to time an outbreak among humans will occur when a virus common in an animal species (such as a pig, bat, or bird) undergoes a change allowing it to spread among humans. This is likely how the new coronavirus outbreak in 2019 began. Genetic fingerprinting of the virus supports this natural explanation.

\section{Is it true that black people cannot be infected by Covid-19?}

No, and by now the answer has become obvious. However, it had been claimed on social media that the virus affected only people of Asian and European descent. Around March 2020, a lot of people falsely thought that melanin would assist with Covid-19 resistance. 
Another misleading claim on the other extreme is to provide statistics of more black people dying from Covid-19 without looking at the socio-economic conditions in which they live. COVID-19 affects everyone.

The belief that Covid-19 does not affect black people has led some to party in large numbers despite the rules, claiming to law enforcement that they are not affected. It is clear that skin colour is not a pivotal factor in determining who gets infected.

\section{Are there presently effective remedies against Covid-19?}

Although the currently available vaccines are highly effective in preventing Covid-19, currently there is no effective drug or vaccine against Covid- 19 for persons already showing symptoms. The immunity given by vaccines takes time to take effect, approximately two weeks after the vaccination. Consequently, vaccines must be administered well in advance to have the desired effect, before it is too late. Vaccines can either prevent one from being infected but there have been cases of infection after vaccination. Fortunately those who fall into the latter category do not develop serious symptoms requiring hospitalization and extreme measures such as ventilation. Most people infected, especially younger people, will recover on their own without medical attention after their body's immune system teaches itself to fight off the disease. Other people, especially older people and people with other conditions, will require supportive care in a hospital, such as supplemental oxygen (and in some cases mechanical ventilation) or anti-clotting drugs. But these measures fight only the symptoms, buying time so that the body can fight the disease off on its own.

Despite this situation, however, a long list of remedies have been advertised on the web claiming to be able to fight off the disease. The list of such remedies is too long to recite here. Modern medicine has developed an elaborate system to vet new remedies, to determine by controlled trials which remedies have been proven safe and effective and which have been not (see appendix $C$ for details). The correct way to deal with recommendations is to follow the advice of a licensed physician or websites maintained by public health authorities. As discussed above, happily vaccines have proved to be a highly effective preventative measure, working better than initial expectations.

\section{What about indigenous knowledge?}

Many widely used and effective modern medicines originated from indigenous knowledge. The cichona bark from South America was used as one of the early cures of malaria. Later it was found that the chemical quinine in this bark was the active ingredient. Artemisinin, a modern anti-malaria drug, derives from traditional Chinese medicine. In South Africa, 'umhlonyane' is one of the indigenous medicines under investigation for use against Covid-19. Traditional knowledge together with modern testing to determine which drugs are safe and effective promise to continue to make new and effective drugs available. 



\section{APPENDIX C}

\section{WHAT IS THE SCIENTIFIC METHOD AND EVIDENCE-BASED MEDICINE?}

\section{History of the scientific method}

The history of the scientific method dates back several thousands of years. One of the oldest medical textbooks is an Egyptian papyrus (the Edwin Smith papyrus) written around $1600 \mathrm{BCE}$. It is assumed that this papyrus is a military surgery manual. It describes around 50 types of injuries. Remarkably, this document follows a modern empirical approach to medical science based on examination, diagnosis, treatment, and prognosis. 
Modern science dates back to the scientific revolution initiated by Nicolaus Copernicus, Johannes Kepler, and Galileo Galilei. These scientists used evidence from astronomical observation to refute the view that the Earth lies at the centre of the Universe. They proposed the alternative view that planets revolve around the Sun. At the time, this scientific revolution was not greeted with universal enthusiasm. The Catholic Church had incorporated the Greek scientist Ptolemy's (100-170) 'geocentric' view as part of its dogma. Galileo Galilei (1564-1642) was threatened by the Church with being burnt alive for contradicting its belief that the Sun revolved around the Earth.

A similar revolution occurred in medicine, when the French biologist and chemist Louis Pasteur (1822-1895) established the connection between micro-organisms and diseases. Louis Pasteur applied the scientific method to disprove the theory of spontaneous generation. According to this theory, living organisms could be created spontaneously from non-living matter, for example air. Pasteur's hypothesis, on the other hand, was that without contamination, living matter (i.e., microorganisms) could not form. To this end, Pasteur boiled meat broth in a gooseneck flask-a flask with a tube in the shape of a gooseneck attached, in order to slow down the flow of air. After boiling, the liquid in the flask remained sterile until it got in touch with the liquid in the tube. This was proof that the germs contained in the air caused the generation and not the air itself (spontaneous generation). An immediate application of the germ theory was the suggestion of a process, still used today, to prevent the bacterial contamination of milk, wine and food, through heating.

This process is now called

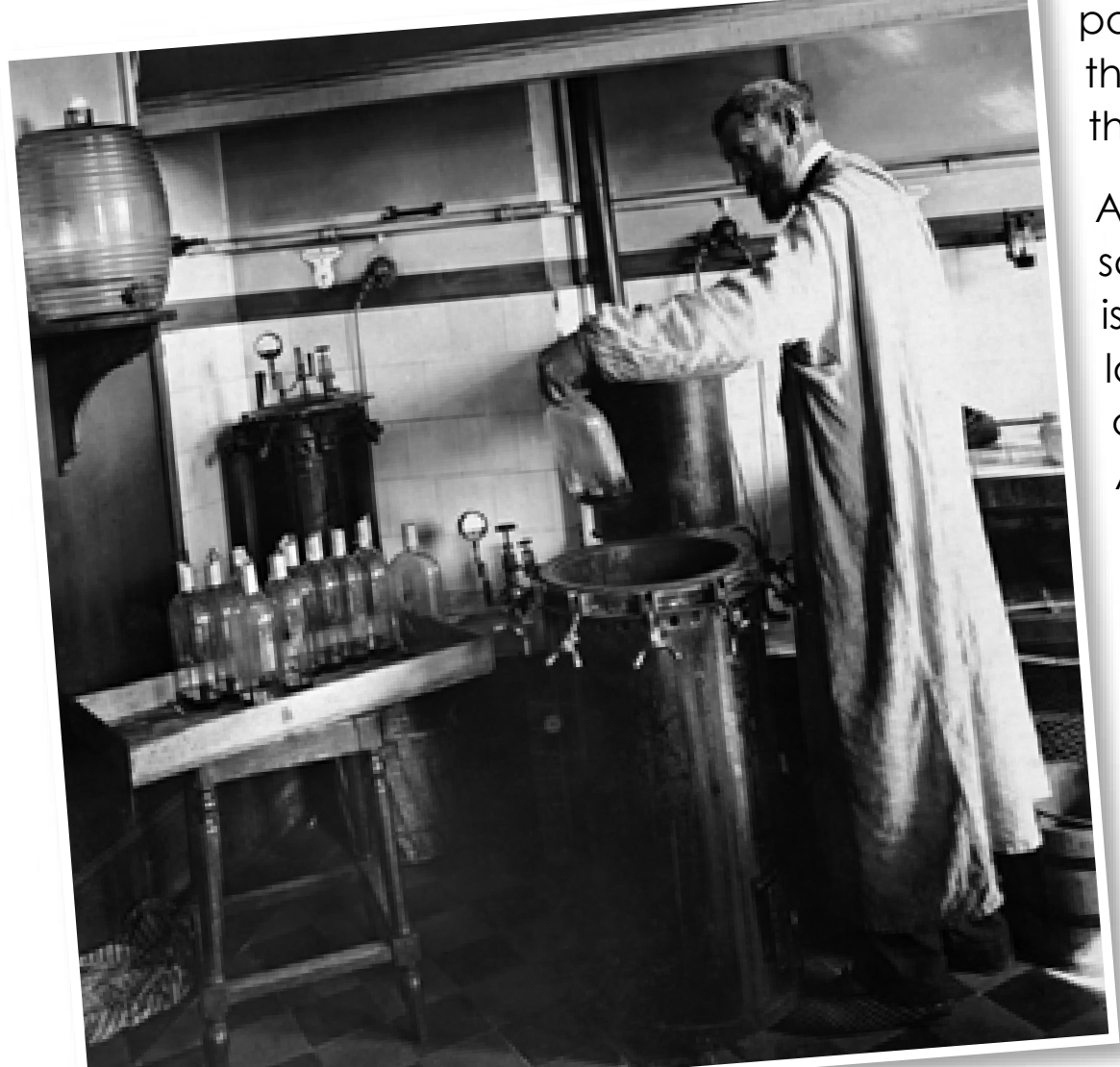
pasteurisation. Pasteur's germ theory is a good example of the scientific method.

A good example of how science moves us forwards is to consider the fact that later scientists, notably amongst them the FrenchAmerican microbiologist Rene Dubos, concluded that the 'germ theory' of disease was indeed correct but incomplete, and that the role of the host was the second-most important factor that determined the ways in which infectious diseases actually manifest in individual members of a population-the 
strength and precise nature of the multi-component immune system, the genetic background, gender, and other variables.

\section{Basic Steps of the Scientific Method}

The scientific method is a strategy to develop models to understand phenomena that we observe. In a nutshell, it consists of the following sequence of five steps:

- We make an observation.

- We ask a question related to the observation.

- We develop a hypothesis that could explain the observation. It is crucial that this hypothesis can be tested.

- We use the hypothesis to make a prediction.

- We test the prediction with the help of an experiment.

Of course, the above steps might have to be repeated, as our initial hypothesis may turn out to be wrong.

\section{In the case of Pasteur's approach described above, the steps were:}

- He observed that microscopic organisms grew in contaminated liquids.

- He asked the question whether contamination and not air could cause the generation of microbes.

- He developed the hypothesis that a boiled liquid would stay uncontaminated if not exposed.

- He made the prediction that by boiling a liquid (the meat broth) and preventing the access of air, germs would not develop. He tested the prediction by boiling the meat broth in a gooseneck bottle. The use of the gooseneck bottle served just the purpose of testing this hypothesis.

It is important to understand that science proceeds according to an hypothesis, which is then validated or not according to the evidence collected. Science is not a belief system. This is why science is the engine of progress and why scientists speak with factual, evidence-based knowledge.

\section{How has the methodology of modern medicine improved on past practices? Intuition vs experiment.}

The scientific method pioneered in medicine by Pasteur immediately led to further advances in medicine. Joseph Lister (1827-1912) was inspired by reading the papers of Pasteur. Lister wanted to understand what caused infections in wounds. He repeated some of Pasteur's experiments and applied the same procedures to wounds. He could prove that it is bacteria entering the wounds that cause the infections that, in many cases, could lead to death. Lister discovered that by 
spraying surgical instruments and cleaning the wounds with a solution of carbolic acid, bacterial infections could be avoided. Needless to say, antiseptic surgery is now the standard of surgery.

For his contribution to medicine, Joseph Lister was honoured by Queen Victoria with the title of Baron. A popular mouthwash "Listerine" is named after him and is still promoted with the slogan: "Kills the germs that cause bad breath"!

\section{What are some past practices in medicine that have turned out to be unfounded?}

The application of the scientific method to medicine also helped to reveal some popular methods and therapies as unfounded. A typical example is the treatment of bloodletting, introduced about 2000 years ago and widely used until the 19th century. According to Claudius Galen (C.129-216/217 CE), a famous physician of the Roman Empire, bloodletting was the 'cure' of choice for fevers, apoplexy, and headaches. This procedure, often performed by barbers, was based on the 'humoural theory', which held that blood, phlegm, black bile, and yellow bile should be balanced in the human body. If one of the 'humours' was out of balance, a disease would result. The theory was aligned to the four fundamental elements of air, water, earth and fire. As a more scientific analysis was applied to this procedure, its use steadily declined.

\section{How do we decide whether a drug is effective?}

During the Age of Navigation, roughly from the 16th to 18th century, about $50 \%$ of sailors were reported to have died of scurvy. Today we know that this disease is caused by a lack of Vitamin C. A Scottish military surgeon aboard a vessel performed an experiment. James Lind divided the crew in six groups and administered different treatments to each group. By monitoring the effects of the different treatments, he observed that the group given lemons and oranges to eat recovered from signs of the scurvy. The publication of these results was probably the first account of a systematic clinical trial.

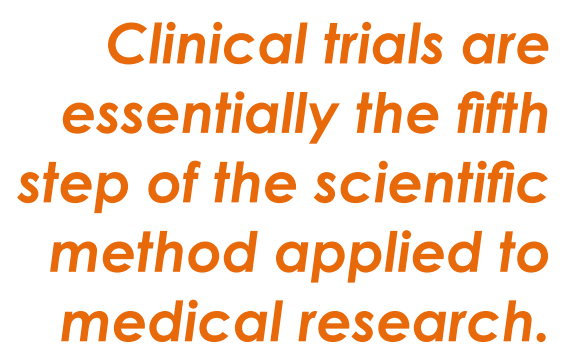

Clinical trials are

Clinical trials are now common practice in medical research. Clinical trials are essentially the fifth step of the scientific method applied to medical research. A clinical trial is an experiment designed to test a hypothesis.

\section{What is the process for developing a new drug? How does one ensure drug safety? And efficacy?}

Before we can test a drug, we need to develop it. This is what is called the preclinical development of new pharmaceuticals. Once a new compound has been found, it is tested on healthy individuals to determine its safety and to determine the proper dosage. Then the new drug is applied to a small number of patients with the 
specific disease. Only after these trials is the drug administered to a large number of patients. Only after three phases of testing have been successful can a drug be considered for licensing, with rare emergency exceptions.

\section{What are some biases to guard against?}

Of course, the best clinical trial is not perfect and some systematic errors, called biases, might contaminate the study. A common bias is the sampling bias. In this case, the pool of patients used for the clinical trial is not representative of the intended study population.

Another bias is called the placebo effect. Often people believe to have been cured after taking a fake drug without the active ingredient. Such a drug is known as a placebo. Consequently, drug trial studies typically use two groups: a control group, which is given the placebo, and another group given the real drug. The patient, and often also their doctor, does not know whether they have received the drug or the placebo. The study is analysed in terms of the difference in outcome between the two groups.

\section{What are some of the ethical dilemmas that arise in drug testing?}

In order to speed up the evaluation of newly developed coronavirus vaccines, some scientists have suggested to deliberately infect volunteers. For a new virus, a clinical trial will typically take at least one year. A carefully designed 'human challenge' trial could reduce the time of the trial to a couple of months. This raises the ethical question about the high risks involved for the volunteers, especially in the case of diseases such as Covid-19, where no effective cure exists yet.

\section{How safe are vaccines?}

As described in detail in Chapter 7, being vaccinated, in order to vaccines reduce the risk of getting a disease. Extensive testing takes place to

The tremendous success of mass vaccination relies on more than a certain threshold fraction of the population test vaccines both for efficacy and safety, and many candidate vaccines do not make it to the market as the result of these tests. Public confidence in the safety of vaccination is crucial.

We mention an important recent success of sustained vaccination. This year the WHO declared Africa free from polio. The eradication of the wild polio virus was only possible because of a systematic vaccination effort spanning over many years. The virus remains endemic only in Afghanistan and Pakistan.

The tremendous success of mass vaccination relies on more than a certain threshold fraction of the population being vaccinated, in order to benefit from the 'herd immunity' explained in Chapter 5. When less than this fraction is vaccinated in some areas or communities, outbreaks can and do occur. Mass vaccination relies 
on widespread public acceptance of vaccines-of their safety, efficacy, and importance. Consequently, false claims that vaccines are not safe can undermine vaccination programs and threaten public health.

The false claims of a link between the measles, mumps, and rubella (MMR) vaccine and autism and its consequences serve as an example. In 1998, the British doctor Andrew Jeremy Wakefield and collaborators published an article in the medical journal, The Lancet, claiming to have discovered such a link. This led to much discussion and the rise of an anti-vaxxer movement. A sharp decline in vaccination uptake followed, leading to a number of outbreaks of measles around the world, with thousands of cases and many deaths. The findings of such a link were subsequently refuted by other researchers and the original article was withdrawn by most of Wakefield's collaborators. It was further uncovered that Wakefield failed to disclose conflicts of interest resulting from his involvement in litigation surrounding the safety of the vaccine.

The anti-vax movement, nevertheless, continues to this day and threatens the widespread acceptance of coronavirus vaccines. In a recent survey held in the United Kingdom, people were asked whether they will undergo the administration of an anti-coronavirus vaccine if one were developed. Apparently, many said 'no', indicating the power of the growing anti-vax movements and the suspicions around the scientific enterprise. 


\section{GLOSSARY}

2019-nCoV Coronavirus disease 2019 (Covid-19) is a contagious disease caused by severe acute respiratory syndrome coronavirus 2 (SARS-CoV-2).

501Y.V2 variant Colloquially known as the 'South African Covid-19 variant' and is a variant of SARS-COV-2, the virus that causes Covid-19.

ACE2 Angiotensin-converting enzyme 2 (ACE2) is an enzyme attached to the cell membranes of cells located in the lungs, arteries, heart, kidney, and intestines.

Acidification The action or process of making or becoming acidic.

Actuarial data Statistics used to calculate various sorts of risk.

Adaptive immune response The adaptive, or acquired, immunity that occurs after exposure to an antigen either from a pathogen or a vaccination.

Adenovirus Adenoviruses are a group of common viruses that infect the lining of your eyes, airways and lungs, intestines, urinary tract, and nervous system.

Aerosol Suspension of fine solid particles or liquid droplets in air or another gas.

Alternative facts Falsehoods, untruths, delusions.

Antagonists A substance that stops the action or effect of another substance.

Antibiotic medicine (such as penicillin or its derivatives) that inhibits the growth of or destroys microorganisms.

Antibody test Antibody or serology tests look for antibodies in your blood to determine if you had a past infection with, for example, the virus that causes Covid-19.

Antiretroviral Antiretroviral drugs are medications for the treatment of infection by retroviruses, primarily HIV.

Antiviral drugs Class of medication used for treating viral infections.

Asymptomatic When no symptoms of disease are evident.

Attenuated vaccine A vaccine containing partial cellular material as opposed to complete cells.

Atypical Not representative of a type, group, or class; not typical.

Azithromycin Antibiotic medication used for the treatment of a number of bacterial infections. 
B.1.1.7 variant Colloquially known as the 'United Kingdom (UK) variant'.

Betacoronavirus One of four genera of coronaviruses.

Bilateral multi-lobular lung Involves lobes in both the right and left lungs.

Biosynthesis Production of complex molecules within living organisms or cells.

Block grant A financial grant from central government, which a local authority can allocate to a wide range of services.

Bond market A marketplace where investors buy debt securities that are brought to the market by either governmental entities or publicly-traded corporations.

Cannula A nasal cannula is a tube used to deliver gas, generally oxygen, through the nostrils or mouth. More generally a cannula can be any tube to provide access to the body, for example to the bloodstream by means of a needle inserted into a vein.

Catchment populations The population of the area served by a facility.

CD4 A large glycoprotein that is found associated with a T cell receptor especially on the surface of helper T cells and that binds to a major histocompatibility complex molecule on the surface of an antigen-presenting cell to facilitate antigen recognition and $T$ cell activation.

Chemotherapy Treatment of disease by introducing chemical substances into the body.

Chloroquine An antimalarial drug.

Chronic infection An infection is chronic when symptoms develop gradually, over weeks or months, and are slow to resolve.

Ciprofloxacin An antibiotic used to treat a number of bacterial infections.

Communicable disease Illness caused by an infectious agent or its toxins that occurs through the direct or indirect transmission of the infectious agent or its products from an infected individual or via an animal, vector or the inanimate environment to a susceptible animal or human host.

Community transmission Describes the situation where an epidemic propagates within a population rather than being brought in by infected people outside the population.

Comorbidities The coexistence of two or more diseases in a person.

Contact tracing Process of identifying all people that a patient has come in contact with. 
Contributory social security Benefits financed through contributions.

Control group The standard or norm to which comparisons are made in an experiment. This group is not exposed to the variable under study.

Convalescent plasma Blood plasma that is obtained from an individual who has recovered from an infectious disease and contains antibodies against the infectious agent.

Cytoplasm Thick solution that fills each cell and is enclosed by the cell membrane.

Denialism Practice of denying the existence, truth, or validity of something despite proof or strong evidence that it is real, true, or valid.

DNA gyrase A bacterial enzyme that causes supercoiling of DNA.

DNA intermediate Viral genome replication where the template is single-stranded RNA, and which proceeds via a double stranded DNA intermediate molecule.

DNA replication Biological process of producing two identical replicas of DNA from one original DNA molecule.

DNA Self-replicating material that is present in nearly all living organisms as the main constituent of chromosomes. It is the carrier of genetic information.

Double-stranded RNA Double-stranded RNA viruses are a polyphyletic group of viruses that have double-stranded genomes made of ribonucleic acid.

Dual healthcare system A situation in which a government-provided healthcare system provides care, and a second system of care exists for those who can pay for private care.

Economic bounce-back To return to the usual economic state or activities after having a problem.

Efficacious Successful in producing a desired or intended result.

Emergency use authorisation (EUA) Authorisation granted to allow the use of a drug before approval.

Endotracheal tube A small, usually plastic tube inserted into the throat through the mouth or nose to maintain an unobstructed passageway for air to flow into the lungs.

Envelope proteins The CoV envelope (E) protein is a small, integral membrane protein involved in several aspects of the virus' life cycle.

Enzyme Substance produced by a living organism that acts as a catalyst to bring about a specific biochemical reaction.

Epicentre Epicentre is the place where something is most common or intense, or where it originated. 
Epidemic Rapid spread of disease to a large number of people in a given population within a short period of time.

Epidemiology The study of the spread and control of infectious diseases in a population and also the prevalence of other diseases, their causes, and other public health matters using statistical methods.

Essential services Services that provide the necessities of life such as healthcare, water, electricity.

Eukaryotic cell Organisms whose cells have a nucleus enclosed within a nuclear envelope.

Experimental group Group of subjects who are exposed to the variable under study. Alongside this group will be the control group against which results are compared.

Exponential growth Growth whose rate becomes ever more rapid in proportion to the growing total number or size.

Fake news False or misleading information presented as news.

Fusion inhibitors Class of antiviral drugs that prevent a virus from entering a cell.

Gastrointestinal Anything related to the digestive system.

Gene A subsequence of the genome, which gets transcribed into RNA. In many cases this RNA, known as messenger RNA (or mRNA) gets translated into a protein whose structure is determined by the sequences of bases of the gene. In other cases, the RNA sequences so transcribed serve functions other than becoming translated into proteins.

Genetic vaccines Approach to immunisation and immunotherapy in which one or more genes that encode proteins of the pathogen are delivered.

Genome An organism's complete set of DNA.

Geometric sequence Ordered list of numbers in which each term after the first is found by multiplying the previous one by a fixed non-zero number, called the common ratio $r$.

Herd immunity Describes when a sufficient fraction of a population (but not the entire population) is no longer susceptible to an infectious disease, either through prior exposure and vaccination, so that the number of persons infected decays with time, leading to the end of the epidemic.

Herpesvirus Any of a group of DNA viruses causing herpes and other diseases.

Human papillomavirus Infection that causes warts in various parts of the body, depending on the strain. 
Hydroxychloroquine A medication used to prevent and treat malaria in areas where malaria remains sensitive to chloroquine.

Hypoxic Deficiency in the amount of oxygen reaching the tissues.

Immune systems Complex network of cells, tissues, organs, and the substances that helps the body fight infections and other diseases.

Immunity Best mix of cells the immune system needs to function at its optimum level.

Immunology Branch of biology that covers the study of immune systems.

Incubation period Period between exposure to an infection and the appearance of the first symptoms.

Infection peak The number of new cases has begun to level off rather than continuing on a sharp upward trajectory.

Infectious (person) Able to pass a disease from one person to another.

Infectious diseases Infectious diseases are disorders caused by organisms-such as bacteria, viruses, fungi or parasites-which can be transferred from one living being to another in any number of modes.

Inhibitor Substance that reduces the activity of another substance.

Insufflation Act of blowing something into a body cavity.

Internal vesicles Structure within or outside a cell, consisting of liquid or cytoplasm enclosed by a lipid bilayer.

Junk status Non-investment grade (bond).

Killed or inactivated vaccine Vaccine consisting of virus particles, bacteria, or other pathogens that have been grown in culture and then killed to destroy disease-producing capacity.

Liquidity How quickly a business can convert its assets into cash.

Live virus vaccines Vaccine made from a virus that has been weakened so it does not cause the disease the virus usually causes.

Lockdown A restriction policy for people or communities to minimise movement, usually due to specific risks to themselves or to others if they can move and interact freely.

Mathematical modelling Description of a system using mathematical concepts and language. 
Mechanical ventilation Assisted ventilation, or intermittent mandatory ventilation, is the medical term for artificial ventilation where mechanical means are used to assist or replace natural breathing.

MERS Middle East respiratory syndrome (MERS) is a contagious, sometimes fatal respiratory illness.

Microbes A microorganism, especially a bacterium, causing disease or fermentation.

Micro-organisms A microscopic organism, which may exist in its single-celled form or a colony of cells.

Monoclonal antibodies Antibody made by cloning a unique white blood cell.

Mucosa The moist, inner lining of some organs and body cavities (such as the nose, mouth, lungs, and stomach).

Multisystem inflammatory condition A condition where different body parts can become inflamed, including the heart, lungs, kidneys, brain, skin, eyes, or gastrointestinal organs.

Mutations An alteration in the genetic material (the genome) of a cell of a living organism or of a virus that is more or less permanent and that can be transmitted.

Narcissism A disorder in which a person has an inflated sense of self-importance.

Nascent Just coming into existence and beginning to display signs of future potential.

Nasopharynx Upper part of the throat behind the nose.

Natural selection Process through which populations of living organisms adapt and change.

Nomenclature System of names or terms, or the rules for forming these terms in a particular field of arts or sciences.

Non-communicable disease A disease that is not passed from one person to another, or from an animal to a person, but rather arises spontaneously, such as heart disease or cancer.

Non-nucleoside reverse transcriptase inhibitors (NNRTIs) Small molecule drugs that bind directly to the active site of HIV-1 reverse transcriptase, disrupting its RNAdependent and DNA-dependent DNA polymerase activities.

Non-pharmaceutical interventions Actions, apart from getting vaccinated and taking medicine, that people and communities can take to help slow the spread of illnesses. 
Nucleic acids Long chainlike molecules composed of a series of nearly identical building blocks called nucleotides.

Nucleoside analogues (NRTIs) An anti-retroviral drug using fake building blocks that cause the replication or reverse transcription of the viral genome to fail

Oropharynx Part of the throat that is at the back of the mouth.

Outbreak A sudden onset of something unwelcome, such as war or disease.

P.1 variant Colloquially known as the 'Brazil variant' and is one of the variants of SARS-COV-2, the virus that causes Covid-19.

Paediatric Relating to dealing with children and their diseases.

Pandemic Epidemic occurring worldwide, or over a very wide area, crossing international boundaries and usually affecting a large number of people.

Pathogens Any organism that can produce disease.

Personal protective equipment (PPE) Protective clothing, helmets, goggles, or other garments or equipment designed to protect the wearer's body from injury or infection.

Phenotypes Refers to the observable physical properties of an organism.

Phylogenetic analyses Uses differences in the genome to map out a family tree of organisms, such as bacteria, viruses, plants, and animals.

Placebo Substance that has no therapeutic effect, used as a control in testing new drugs.

Placebo effect Positive effect on a person's health experienced after taking a placebo. It is triggered by the person's belief in the benefit from the treatment and their expectation of feeling better

Post-attachment inhibitors Class of drugs that bind to the CD4 receptor on a host CD4 cell.

Poxvirus infections Brick or oval-shaped viruses with large double-stranded DNA genomes.

Pre-exposure prophylactics Medications used to prevent the spread of disease in people who have not yet been exposed to a disease-causing agent, usually a virus.

Pre-symptomatic transmission Phase when an individual is infected and may be shedding virus but has not yet developed symptoms. 
Protein A macromolecule formed as a chain of amino acids linked through peptide bonds. Proteins are encoded in the DNA (or RNA in the cases of some viruses). This code gets 'transcribed' into messenger RNA molecules, which in turn get 'translated' into proteins in the ribosomes of a cell.

Protein-synthesis Creation of proteins by cells that uses DNA, RNA, and various enzymes.

Protracted period Drawn out or lengthened in time.

Quarantine Restriction on the movement of people, animals and goods which is intended to prevent the spread of disease or pests.

$\mathbf{R}$ number Way of rating coronavirus or any disease's ability to spread. $R$ is the number of people that one infected person will pass on a virus to, on average.

$\mathbf{R}_{0}$ Mathematical term that indicates how contagious an infectious disease is. It is also referred to as the reproduction number.

Recombinant vaccines Products of genetic engineering, where a harmless agent, such as yeast, is programmed to produce antigens of harmful pathogens.

Recovery trial Large-enrolment clinical trial of possible treatments for people in the United Kingdom admitted to hospital with severe Covid-19 infection.

Regulatory proteins Any protein that influences the regions of a DNA molecule that are transcribed by RNA polymerase during the process of transcription.

Respiratory diseases Diseases of the airways and other structures of the lung.

Retroviral integrase Enzyme produced by a retrovirus that integrates-forms covalent links between-it's genetic information into that of the host cell it infects.

Retroviruses Type of virus that inserts a copy of its RNA genome into the DNA of a host cell that it invades, thus changing the genome of that cell.

Reverse transcription Process in cells by which an enzyme makes a copy of DNA from RNA. The enzyme that makes the DNA copy is called reverse transcriptase.

RNA elongation Stage when the RNA strand gets longer, due to the addition of new nucleotides.

RNA replicating Process by which new copies of genome-length RNAs are made.

RNA virus Virus that has RNA (ribonucleic acid) as its genetic material.

RT-PCR Reverse transcription polymerase chain reaction is a laboratory technique combining reverse transcription of RNA into DNA and amplification of specific DNA targets using polymerase chain reaction. 
SARS A contagious and sometimes fatal respiratory illness caused by a coronavirus.

SARS-CoV-2 Member of a large family of viruses called coronaviruses. These viruses can infect people and some animals. This virus causes the Covid-19 disease.

Second wave A phenomenon of infections that can develop during a pandemic. The disease infects one group of people first. Infections appear to decrease. And then, infections increase in a different part of the population, resulting in a second wave of infections.

Secondary bond market Also called the aftermarket and follow-on public offering, is the financial market in which previously issued financial instruments such as stock, bonds, options, and futures are bought and sold.

Serological test Blood tests that look for antibodies in blood.

Serotype A distinct variation within a species of bacteria or virus or among immune cells of different individuals.

Shelter-in-place order An official order, issued during an emergency, that directs people to stay in the indoor place or building that they already occupy and not to leave unless absolutely necessary.

Single-stranded RNA virus Virus whose genetic information consists of a single strand of RNA that is the positive (or sense) strand which encodes mRNA (messenger RNA) and protein.

Social distancing A set of non-pharmaceutical interventions or measures intended to prevent the spread of a contagious disease by maintaining a physical distance between people and reducing the number of times people come into close contact with each other.

Solidarity trial International clinical trial to help find an effective treatment for Covid-19, launched by the World Health Organization and partners. It is one of the largest international randomised trials for Covid-19 treatments, enrolling almost 12 000 patients in 500 hospital sites in over 30 countries.

Spike protein These spikes protrude from the outside of coronaviruses and help them infect cells.

Sputum Mixture of saliva and mucus coughed up from the respiratory tract, typically as a result of infection or other disease and often examined microscopically to aid medical diagnosis.

State of emergency Situation of national danger or disaster in which a government suspends normal constitutional procedures in order to regain control.

Stigmatisation The action of describing or regarding someone or something as worthy of disgrace or great disapproval. 
Stimulus plans A stimulus package is a package of economic measures a government invokes to stimulate a floundering economy.

Structural unemployment Structural unemployment is a form of involuntary unemployment caused by a mismatch between the skills that workers in the economy can offer, and the skills demanded of workers by employers.

Subunit vaccines $A$ vaccine that presents one or more antigens to the immune system without introducing pathogen particles, whole or otherwise.

Super-spreader events Large events, attended by a large number of people, where a greater amount of transmission than would be expected occurs.

Susceptible Likely or liable to be influenced or harmed by a particular thing.

Symbiotic relationship The living together in more or less intimate association or close union of two dissimilar organisms.

Synthetic compounds Substance that is man-made (by synthesis), rather than being produced by nature.

T cell A type of white blood cell. T cells are part of the immune system and develop from stem cells in the bone marrow.

Transmission A passage or transfer, as of a disease from one individual to another. Vaccine A biological preparation that provides active acquired immunity to a particular infectious disease.

Ventilatory support The patient is on a machine that helps them breathe. A tube is put in through their nose or mouth into the trachea (windpipe). It is attached to a ventilator.

Variant A form or version of something that differs in some respect from other forms of the same thing or from a standard.

Viral evolution Sub-field of evolutionary biology and virology that is specifically concerned with the evolution of viruses.

Viral genome A virus has either DNA or RNA genes and is called a DNA virus or a RNA virus. The genome includes both the genes and the non-coding sequences of the DNA/RNA.

Viral protein Protein generated by a virus.

Viral test Checks specimens from your nose or your mouth to find out if you are currently infected with SARS-CoV-2, the virus that causes Covid.

Virulent A virulent disease or poison is dangerous and spreads or affects people very quickly. 
Virus particle A small particle that contains certain proteins from the outer coat of a virus.

Virus-encoded proteins Proteins generated by a virus.

Wet market A marketplace selling fresh meat, fish, produce, and other perishable goods as distinguished from 'dry markets' that sell durable goods such as fabric and electronics.

Zoonotic Caused by germs that spread between animals and people. 


\section{Essential facts about Covid-19: the}

disease, the responses, and an

\section{uncertain future. For South African}

\section{learners, teachers, and the general public}

Bucher, Martin (ed)

Academy of Science of South Africa (ASSAf)

Cite: Academy of Science of South Africa (ASSAf), Bucher M (ed), Mall AS (ed), (2021). Essential facts about Covid-19: the disease, the responses, and an uncertain future. For

South African learners, teachers, and the general public. [Online] Available at: DOI http://dx.doi.org/10.17159/assaf. http://hdl.handle.net/20.500.11911/194

Downloaded from ASSAf Research Repository, Academy of Science of South Africa (ASSAf) 